\title{
THE EXPERIENCES OF ELEMENTARY MUSIC TEACHERS IN A COLLABORATIVE TEACHER STUDY GROUP
}

\author{
by
}

\author{
Ann Marie Stanley \\ A dissertation submitted in partial fulfillment \\ of the requirements for the degree of \\ Doctor of Philosophy \\ (Music: Music Education) \\ in The University of Michigan \\ 2009
}

Doctoral Committee:

Associate Professor Colleen M. Conway, Chair

Professor Magdalene Lampert

Professor Marie F. McCarthy

Associate Professor Stephanie D. Teasley

Associate Professor Elizabeth Anne Younker 
(C) Ann Marie Stanley

2009 


\section{ACKNOWLEDGEMENTS}

I would like to thank Dr. Colleen Conway for her support and guidance, which started the very first moment I met her and continues to the present. I am grateful for the mentorship and look forward to many years of collaboration.

I would also like to thank Drs. Betty Anne Younker, Marie McCarthy, Stephanie Teasley, and Magdalene Lampert for being influential role models. Each of you taught a course at the University of Michigan that played a big part in how I think about teaching and learning. I am lucky to have had a graduate school experience with professors of your caliber and I hope you will see your influence on these pages.

I would like to thank my family and friends for your help and encouragement. Mom, Dad, Kristi, Andrew, Oliver, Linden, and Dan: you all kept me happy throughout. Thanks also go to my colleagues and friends at the Eastman School of Music who have supported my endeavors.

I am very grateful to my friend and closest colleague at U of M, John Eros. We've come so far since meeting on the first day of graduate school! We now have a lifelong friendship and bond. I would also especially like to thank my aunt, Ms. Phyllis Stanley, for hours of patient editing and positive feedback.

Finally, I thank the CTSG-Marlene, Andrea, and Karly_-for making this project possible and for doing wonderful things for kids and music. 


\section{CONTENTS}

ACKNOWLEDGEMENTS ......................................................................... ii

LIST OF APPENDICES ....................................................................... viii

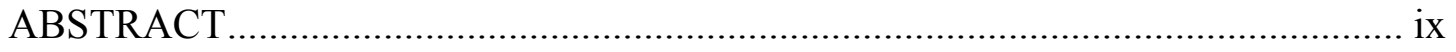

\section{CHAPTER}

1. A COMMUNITY: INTRODUCTION ...............................................

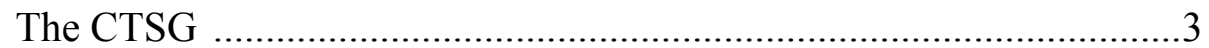

Purpose Statement and Research Questions ...................................4

Music Teaching Practice and Theories of Learning ...........................5

Teachers and Professional Development ..................................... 9

Professional Development in Communities ....................................11

Definitions of Collaboration ........................................................ 12

Definition of Collaboration for This Study ...................................14

Collaboration in Music Education ................................................15

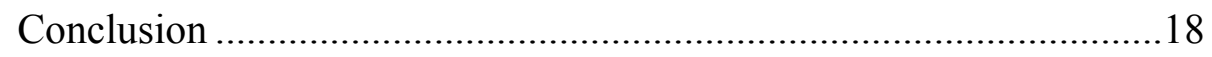

2. A COMPILATION: REVIEW OF THE LITERATURE ....................... 20

Perspectives on Teacher Communities ...........................................21 
Issues in Teacher Professional

Development Communities

Issues in Music Teacher Professional

Development Communities

Characteristics of Successful Professional

Development Communities

Collaboration in General Education 50

Collaboration in Music Education .68

Collaboration in Student Chamber Music Groups 82

Conclusion .88

3. THE CONSTRUCTION: METHODOLOGY

Participants and Site

The CTSG Community .93

Study Design .95

Pilot .97

Sources of Data 102

Timeline and Procedures 105

Analysis 106

Goodness Criteria 108

Organization of Findings 111

4. CHRONICLES: PARTICIPANT PROFILES 114 
Marlene's Story: Introduction

Marlene's Story, Part One: At The Beginning …………………......116

Marlene's Story, Part Two: CTSG Midpoint ……………………......125

Marlene's Story, Part Three: At the End of the CTSG ……..............132

Summary of Marlene's Story …………………………………….....137

Connection to Andrea's Story ………………………………...........140

Andrea's Story: Introduction .........................................................140

Andrea's Story, Part One: At The Beginning ......................................141

Andrea's Story, Part Two: CTSG Midpoint …………………….......155

Andrea's Story, Part Three: At the End of the CTSG .......................164

Summary of Andrea's Story ……………………………................169

Connection to Karly's Story ……………………………................170

Karly's Story: Introduction .........................................................170

Karly's Story, Part One: At The Beginning …………………….......171

Karly’s Story, Part Two: CTSG Midpoint …………………...............186

Karly's Story, Part Three: At the End of the CTSG ……………......194

Summary of Karly's Story ................................................................197

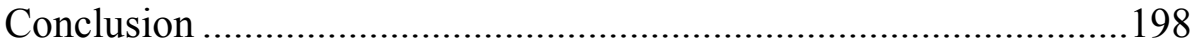

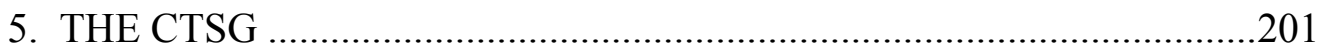

CTSG: Professional Development That Supports

Teacher Learning and Change in Practice ..........................................202

CTSG as Professional Development That Combats Isolation ..........227 
Conclusion

6. COLLABORATION

Structure of the Chapter

Definitions of Collaboration Constructed by the CTSG: Introduction

Meeting One

Meeting Two 240

Beginnings of a Socially Constructed Definition 242

Meeting Three 244

Meeting Four 249

Meeting Five 255

Meeting Six 259

Wrap-Up Meeting 260

A Socially Constructed and Shared Definition of Collaboration .....265

Vignettes and Principles of Collaboration 268

Vignette one: Karly's class and

"Amazing Grace" 269

Summary 277

Vignette two: Marlene's class and

"Hot Cross Buns." 278

Summary 281 
Vignette three: Andrea's class and

"Sally Go 'Round the Sun." .283

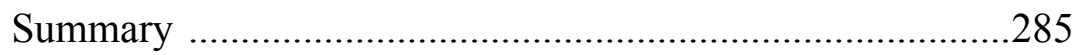

Connection of Principles to Past Research ........................285

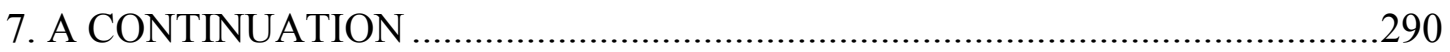

Summary of the Study ....................................................................290

Definition of a Collaborative Teacher Study

Group (CTSG), Participants, and Site .........................................291

Related Literature in Teacher Community ..................................292

Collaboration in Education ........................................................292

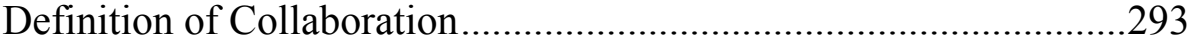

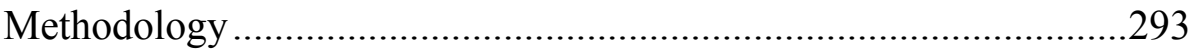

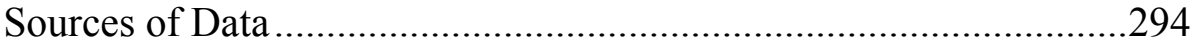

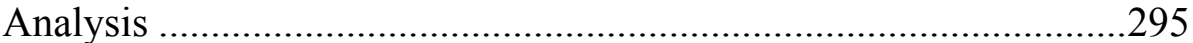

Summary of Findings..............................................................295

Implications for Practice in Teacher Collaboration and Professional Development ....................................................298

Implications for Practice in Student Musical Collaboration.............307

Suggestions for Future Research ................................................308

A Final Conversation ................................................................ 311

APPENDICES

REFERENCES 


\section{LIST OF APPENDICES}

Appendix A Collaborative Consultancy Protocol 314

Appendix B Dilemma Consultancy Protocol 315

Appendix C Representative Questions for Semi-structured Interviews with CTSG Participants 316

$\begin{array}{lll}\text { Appendix D Coding Categories } & 317\end{array}$

Appendix E Strategies For the Collaborative Music Classroom 320

Appendix F Institutional Review Board Exemption Letter 324 


\begin{abstract}
The purpose of this study was to examine the experiences of three elementary music teachers in a collaborative teacher study group (CTSG). The CTSG met seven times to discuss elementary music student collaboration by analyzing video from each participant's classroom.

The study was a social constructivist inquiry. Using interview and meeting transcripts, the researcher investigated how the CTSG members' perceptions were affected by group interactions. The evolution of the group's definitions of elementary music student collaboration was traced and documented.

Research questions were: How do the participants describe their experience in the CTSG? How has the focus on collaboration in the CTSG changed their teaching practice? What can these music teachers tell other music educators about collaboration?

Four elements of the CTSG emerged as central to the participants' experiences: the group's collectively generated knowledge, the necessity of video, the importance of a protocol in classroom analysis, and creating a definition of student work in music.

The participants indicated they had increased confidence in professional knowledge through the opportunity to share teaching expertise, an expanded vocabulary to analyze and describe student behavior, a more habitual reflective examination of
\end{abstract}


teaching, an expanded understanding of student collaboration, and a knowledge of the teacher's role in facilitating student collaboration.

Three principles of collaboration were extracted from the group's definitions of collaboration in elementary music:

1. Collaboration facilitates student self-expression and independence.

2. Students who are collaborating share goals. The teacher allows space for, or guides students in creating, productive student-student interactions.

3. Collaboration between students and teachers facilitates movement toward a shared goal. Teachers provide necessary background skills, create student buy-in for the goal, and then fade away allowing students to take ownership.

This study has implications for how professional development may be structured as a non-hierarchical community that includes protocol-oriented analysis of teaching and learning, and focuses on the creation of locally meaningful knowledge. The principles of collaboration may be located in the teaching and learning practice of others, including students in all areas of music education. Evidence of and for student musical collaboration is a rich area for future study. 


\section{Chapter 1}

\section{A COMMUNITY: INTRODUCTION}

Picture a group of three elementary music teachers and a university professor who meet weekly to eat, talk about music teaching practice, share stories from the week, and laugh. The group is close-knit; they enjoy getting together on Thursday afternoons to watch video from the teachers' classrooms.

The teachers gather weekly because they have committed to making their classrooms places where students learn through collaborative, active music-making. They want to figure out ways to help their students to infuse class projects with creativity and energy. These teachers don't want to create an artificial or manufactured music environment for their students, with worksheets, or karaoke-style soundtracks. Instead, they want their students to watch, listen to, and respond to one another like musicians in a jazz quintet or symphony orchestra. All of them share a belief in Bruner's (1960) assertion that "any subject can be taught effectively in some intellectually honest form to any child at any stage of development" (p. 33). They want even their youngest students to learn musical skills and make real music, not just read about it or do make-believe, watered-down musical activities.

None of the teachers has all the answers. In fact, all of them have struggled to incorporate their ideals within the real-world strictures of public school music. What they want for their students is difficult to make manifest in practice. The meetings help the 
teachers share ideas and strategize within the context of real teaching situations. Because the teachers bring video of their classrooms to the meeting, the group can talk realistically about each teacher's specific scenario and the students on video.

Although the teachers share a belief in the value of active music-making in general music, they have different worldviews, backgrounds, and teaching assignments.

Marlene (pseudonyms have been given to the elementary music teacher participants) is educated in Orff-Schulwerk and Kodàly methods, and has taught successfully for twelve years in districts in other states and her native Puerto Rico. This is her first year teaching in a large urban district: she is itinerant, which means she travels to three schools weekly and teaches from a cart. Despite Marlene's extensive teaching experience, this year finds her struggling to establish feelings of safety, self-expression, and community in her music classes.

Andrea is a third-year teacher who would like to cede a bit more control to her students, as she teaches in a K-5 school and recognizes her students are growing more capable every year. But she loves direct instruction, and lesson plans which incorporate highly directed student practice and response. She feels that in these lessons, she can control the learning environment well and the students in turn feel safe and successful. Andrea teaches in a wealthy suburban district with a very homogenous population.

Karly is a third-year teacher who often emphasizes tonal and rhythmic audiation according to Music Learning Theory (Gordon, 1997). She teaches ten classes per day in her inner suburban school, all second grade and younger. She struggles with the amount of energy and memory it takes to keep all her students engaged and remember their 
individual needs. Many of her students are English language learners or have Individual Education Plans (IEPs).

The CTSG

What I have described above is our collaborative teacher study group (CTSG). We met together throughout the spring of 2008. I am the university professor who organized the CTSG because I wanted to create a different type of professional development for teachers. I wanted the CTSG to be truly collaborative, meaning we would learn through interaction and sharing ideas. I wanted to create a learning environment for teachers conducive to constructing deeper, more personal understandings of music education. And I wanted to be a learner myself as a member of the CTSG, not just automatically in charge because I was facilitating the meetings.

I felt the CTSG should be organized around one topic of study, so I set up the CTSG to be a place where we would study elementary music student collaboration. I had studied student musical collaboration before (Stanley, 2007) and felt it was a natural fit with the teaching and learning goals espoused by all of us. Also, I thought our collaborating to discuss student collaboration would give us an interesting perspective. In my view, a collaborative music classroom would have much in common with a collaborative teacher study group, or any other place where people learn through interaction and sharing ideas. I wondered if the teachers' own experience with collaboration in the CTSG would result in a trickle-down effect: would they be more likely to help their students collaborate if they experienced the value of it themselves, as learners in the CTSG? 
I also wanted to explore the CTSG from the inside out, as a researcher. I decided to take three paths of exploration. First, I wanted to know how Marlene, Andrea, and Karly would describe their experiences in the CTSG, because a collaborative teacher study group was a new form of professional development for all of us. Second, I wanted to know how the focus on collaboration in the CTSG might change their teaching practice. Third, I wanted to preserve everything we found out together about student musical collaboration so we could tell other music teachers about what we learned.

While there is no superior vantage point from which to survey the intrinsic beliefs, perceptions, and learning of an individual or a community, I knew that my roles of CTSG facilitator and learner would privilege me to understand what happened in our meetings, or as a result of our meetings. I feel fortunate here to be able to recount and interpret the journey along these three paths of exploration.

\section{Purpose Statement and Research Questions}

The purpose of this study was to examine the experiences of three elementary music teachers and a researcher involved in a collaborative teacher study group (CTSG) designed specifically to focus on student collaboration in elementary music classrooms. The research questions were:

1. How do the participants describe their experience in the CTSG?

2. How has the focus on collaboration in the CTSG changed the teaching practice of these teachers?

3. What can these music teachers tell other music educators about collaboration? 


\section{Music Teaching Practice and Theories of Learning}

To understand the symbiotic relationship between collaboration and learning in music, I turned to recent research on how people learn, and applied findings from that body of literature to music education.

Papert (1993) coined the term "instructionism" in a critique of the traditional view of schooling. Instructionism implies a view of knowledge as a collection of facts and procedures; schooling is intended to impart these facts and procedures to students in an expedient, teacher-controlled, and testable manner. In response to instructionism, researchers investigated alternative models of learning in the 1970s and 1980s. By the 1990s a different model of learning emerged and was codified by educational researchers and psychologists, the basic tenets of which were published in a book called How people learn: Brain, mind, experience and school (Bransford, Brown, \& Cocking, 2000). The salient points of this model are:

1. Deep conceptual understanding is necessary to allow students to transfer knowledge to other fields, and apply facts and procedures to the real world.

2. Schools should focus on the learning process of students, rather than concentrating on ways to make teachers teach better or more efficiently.

3. Schools should be learning environments that relate to authentic realworld practices needed to function as expert adults, with a strong emphasis on long-term, collaborative, project-based learning and social interaction.

4. Teaching must access students' prior knowledge and build upon it. 
5. Students learn best when they are provide opportunity to express and reflect upon knowledge, through conversation or creating something new.

The view that learning best takes place within the context of authentic, real-world environments is not new to music educators. Many music teachers strive to create learning environments that offer hands-on engagement with music. The nine National Standards for Music Education (MENC, 1994) involve singing, performing on instruments, improvising melodies, variations, and accompaniments, composing and arranging music, reading and notating music, listening to, analyzing, and describing music, evaluating music and music performances, understanding relationships between music and other arts and disciplines, and understanding music in relation to history and culture. These goals definitely reflect the real-world practices of musicians, and therefore are in agreement with the way people learn.

However, some music teaching practices seem to be in conflict with the way people learn. There are common music teaching strategies that leave little time for constructing deeper conceptual knowledge, reflecting on knowledge gained, or collaborating with peers. For example, some music classrooms and rehearsals operate using direct, systematic instruction (Rosenshine, Froelich, \& Fakhouri, 2002), defined as incorporating six steps in sequential order: (a) review of previously learned skills and material; (b) statement of lesson objectives, and instructions about how to achieve the objectives, with related questions posed by the instructor; (c) students are led in guided practice to achieve objectives, with teacher modeling and teacher-led discussion; (d) teacher provides clear feedback and re-teaches if necessary; (e) independent practice, 
which may occur at home; and (f) review of older objectives achieved which may be incorporated in new lesson plans.

The direct instruction approach to music education, rooted in research in the teaching of reading and mathematics, has a large body of literature to support it. The "Systematic Instruction" chapter (Rosenshine, Froelich, \& Fakhouri, 2002) in the Handbook for music teaching and learning (Colwell \& Richardson, 2002) is a summary of a great deal of research pointing to its advantages. Direct and systematic instruction is not necessarily "instructionist," nor does it preclude collaborative activities. For Rosenshine, Froelich, and Fakhouri (2002) "student-initiated, cooperative learning situations" (p. 300) and "performer-led decisions" (p. 309) are valuable components of a rehearsal. But they also say peer collaboration is "complicated and time-consuming" (p. 310) and acknowledge little music education research exists on this topic.

Elementary music teachers may feel a dissonance between the two models. The learning environment codified by Bransford, Brown, and Cocking (2000) places an emphasis on independent decision-making, as students work together to identify and solve problems. In contrast, the direct instruction environment relies on teacher-stated objectives followed by teacher instructions regarding how and what to practice to achieve said objectives.

The tension many teachers feel is understandable. On one hand, the conductor-led ensemble is an immutable part of music education in our culture. Direct instruction in precise musical skills is well regarded by teachers. On the other hand, many elementary music teachers see the value of constructivist, inquiry-based learning, reliant on student 
interaction and communication. Teachers may feel their students are more motivated by projects that enable discovery and creativity with peers.

Like many important issues in education, resolution of the dissonance probably lies somewhere on a continuum. Both ends of the continuum-completely teacherdirected lessons, and completely student-constructed, free-form discovery—are impractical. In looking at the range between "total teacher control" and "total student control," Schwartz and Bransford (1998) review a number of studies and conclude "guided discovery" and "scaffolded inquiry" are more effective than totally unconstrained environments.

Researchers of learning environments have had difficulty linking teacher behavior and instruction style to student outcome, which further complicates the choices for teachers. In Duke's (1999) review of 86 studies published in the field of instructional effectiveness in music teaching, he found that only 13 had attempted to measure related student achievement. Only one study was able to connect the behavior of teachers with student performance quality. Sink (2002) also stated that the outcomes of teacher behaviors in direct instruction have yet to be tied conclusively to any increase in musical achievement, or movement toward independent musicianship.

For music teachers striving to create a learning environment supporting student understanding, the choices are many. What is guided discovery or scaffolded inquiry in music? Where is the middle ground between direct instruction and too much freedom? How do students move beyond imitative, rote repetition and start to understand the rich fullness of creative music-making with others? Can a classroom be a community? 
Music teachers committed to professional growth should be able to examine these complicated issues in substantial, thoughtful ways. Improving instruction and enhancing student learning are worthy ends, and will be reliant on clarifying ill-defined concepts, such as the role of collaboration in the learning environment. But what can music teachers do to work toward such complex goals? How and when are they going to do it? Teachers will not be able to do this one-by-one, alone. Progress may become compromised in an isolated environment. It seems likely that teachers who receive support from others in a collaborative environment will be better able to scrutinize music teaching practice and make systemic changes.

\section{Teachers and Professional Development}

The role of music educators in today's educational climate is rapidly changing; researchers have argued that music teachers need to continue to grow and improve in order to better meet the needs of students. Barrett (2006) states "overviews of what [music] teachers should know and be able to do... can barely keep up with the shifting demands of what teachers are called to do" (p. 20). She goes on to describe the reformminded music teacher as making a commitment to student learning "informed by the continual adjustment, invention, and reconstruction" of practice (p. 21). Shuler (1995) also describes the need for teachers to adapt their practice over their years in the profession: "over the span of a career...there will be many changes in the nature of music, the nature of students, and the nature of schools. Even well-prepared teachers must therefore learn to adapt to change" (p. 10).

Some writers, favoring a "reconceptualized music curriculum" (Hanley and Montgomery, 2002; 2005), have rejected some traditional music teaching practice as 
inconsistent with modern learning theory and the changing needs of contemporary schools. Shifts in enrollment, new or altered course offerings, and varying student and community needs are just a few of the modifications in their job descriptions with which music teachers must cope. Music teachers cite the following among long-term challenges to the quality of their teaching: time management, advocacy, isolation, curriculum concerns, scheduling, keeping up with educational trends, and finding time for one's own music growth (Conway, 2003). It is not surprising that music teachers often feel change in teaching practice is necessary, and at the same time are unsure how to accomplish it.

Professional development is the conventional mechanism for instigating change in teaching practice, but the amount and quality of professional development available to teachers is "woefully inadequate" (Borko, 2004, p. 3). Feiman-Nemser (2001) likewise calls for meaningful, sustained teacher learning: "if we want schools to produce more powerful learning on the part of students, we have to offer more powerful learning opportunities to teachers" (p. 1014). However, some music teachers have little professional development specific to music at all, and a balance between content-specific and general professional development is difficult to achieve (Conway, Hibbard, Albert, \& Hourigan, 2005a; 2005b).

Even when music-specific professional development is available to teachers, there is a surprising lack of research-based evidence that it will be productive or worthwhile. In a review of the literature on music teacher professional development, Bauer (2007) criticizes extant research as lacking "broad perspectives and detailed understandings of this complex phenomenon" (p. 20) and calls for more investigation of the efficacy of all components and types of professional development. 


\section{Professional Development in Communities}

The literature on learning in groups and the literature on professional

development both indicate that powerful learning occurs in communities, and that music teachers benefit from this connection with others. Conway, Albert, Hibbard, and Hourigan (2005a and 2005b), in questioning the prevalent model of one-day conference and workshop professional development, ask:

What about developing sharing communities of arts teachers who, as the real experts in many cases, get together to problem solve and exchange ideas? What about ongoing, regular workshops for arts educators, where progress and change is shared among the group? Somehow, we need to get beyond "token" days or hours of sharing good ideas and move toward meaningful experiences where the voice of the teacher and the effects on students are being discussed and felt. (p. 8)

Hammel (2007) supports the view of the researchers above. She states: "Music educators may feel isolated from other professionals in their schools. For example, music educators have been asked to attend in-service sessions and implement schoolwide assessment models that do not relate at all to music curricula" (p. 23).

Feiman-Nemser (2001) calls "serious ongoing conversation... in communities of practice" (p. 1042) an important element of professional development:

[This conversation] focuses on the particulars of teaching, learning, subject matter, and students. By engaging in professional discourse with like-minded colleagues grounded in the content and tasks of teaching and learning, teachers can deepen knowledge of subject matter and curriculum, refine their instructional repertoire, hone their inquiry skills, and become critical colleagues. (p. 1042)

The connection between communities of teachers and teacher learning has been a fruitful area of exploration in the field of teacher professional development research. However, despite the calls by practicing teachers and educational researchers for an increased presence and importance of meaningful teacher community structures within 
professional development, some teachers have never participated in this sort of group work. The three teachers and I in the CTSG have participated only rarely in what FeimanNemser would call "serious ongoing conversation" intended to hone knowledge of music or music teaching practice. In this study I examined a music teacher community as a form of professional development; we shared an ongoing conversation about aspects of student collaboration in the elementary music classroom.

\section{Definitions of Collaboration}

The idea that people working together will come to learn and share understandings has a long, well-documented history in education and cognitive psychology. Called, variously, "social constructivism" (Rogoff, 1990), "situated cognition" (Brown, Collins, \& Duguid, 1989), or "knowledge within a community of practice" (Lave \& Wenger, 1991), this sociocultural approach to learning asserts that socially shared activities lead to shared processes of meaning-making. The connection between social interaction and cognitive development has its roots in some of the twentieth century's leading perspectives on learning (Dewey, 1938; Mead, 1934; Rogoff, 1990; Vygotsky, 1978). Azmitia and Perlmutter (1989) summarized that "social contexts can facilitate learning because partners often provide new information, define a problem in such a way that it becomes manageable, and generate a discussion that culminates in the selection of the best strategy and solution" (p. 90).

Collaboration has been defined as a setting in which people negotiate, construct and share relevant meanings, participate in coordinated, synchronous activity and pursue a continued attempt to maintain new concepts (Roschelle \& Teasley, 1995). Researchers have studied how to harness collaboration as a powerful learning mechanism, within the 
classroom and within the professional development setting (Gokhale, 1995; Luce, 2001).

Proponents of learning through collaboration assert that people working together toward a common goal are stimulated to create consensus and share knowledge; through the process, people learn more about a topic as a "community of knowledgeable peers" (Bruffee, 1999, p. xii) than they would as a lone learner.

Researchers have closely examined aspects of different types of collaborative work to determine how and why cognition seems to be enhanced in group problemsolving, or when and why two (or three or four) heads seem to be better than one. What is further defined in this study is how teacher learning is affected by collaboration with knowledgeable peers.

In this study, I also explore the role and characteristics of collaboration within the elementary music classroom. Collaboration does not just occur in chamber music or small group work. Dillenbourg (1999) suggests that a class of 25 can collaborate as long as interactivity with peers influences the students' cognitive processes, and as long as there is a degree of negotiation and synchronous action.

While the review of the literature in Chapter Two elucidates the aspects of collaboration that are problematic or advantageous in classroom settings, this quote from Rogoff (1990) argues convincingly for the inclusion of collaboration in education:

Children's participation in communicative processes is the foundation on which they build their understanding ... as they are assisted in problem solving, they are involved in the views and understanding of the skilled partner, in the process of stretching their concepts to find a common ground; as they collaborate and argue with others, they consider new alternatives and recast their ideas to communicate or to convince. In these activities, children advance their ideas in the process of participation. It is not a matter of bringing to the internal plane a product that was produced externally. It is a matter of social engagement that leaves the individual changed. (p. 195-196) 


\section{Definition of Collaboration for This Study}

There is a lack of consensus in the literature on the definition of collaboration.

While I thought we in the CTSG might create our own definition, I chose Roschelle and Teasley's (1995) as the most pertinent to support the work of this study:

Collaboration is a process by which individuals negotiate and share meanings relevant to the problem-solving task at hand...Collaboration is a coordinated, synchronous activity that is the result of a continued attempt to construct and maintain a shared conception. (p. 70)

Cooperation and collaboration are sometimes used interchangeably. However, according to Dillenbourg (1999), in cooperation, work is split between group members and completed individually. The final product is assembled from these individual outputs. Collaboration, by contrast, is characterized by symmetry of action (each person has the same possibility of actions, not merely one assigned role or task), and approximate symmetry of knowledge and status within the group. A collaborative group will interact repeatedly and synchronously to establish and maintain mutual awareness of shared goals, to reason, and to influence one another. The achievement of shared understanding, or intersubjectivity, is the goal.

Dillenbourg concedes that division of labor may occur in collaboration as well as cooperation, but that in collaboration the division is unstable, and partners will take turns at the "task-" and "meta-levels." In other words, one person may work on the task while other group members operate at the monitoring level, but "roles may shift every few minutes, with the regulator becoming the regulated, while cooperation refers to a more fixed division of labor generally made explicit at the outset" (Dillenbourg, 1999). 


\section{Collaboration in Music Education}

The more I read about the role of collaboration in learning, and the definitions of collaboration in the literature, the more I am convinced that music-making is an innately collaborative endeavor. To help me make this point compellingly, I considered each element of the definition of collaboration and compared them with what I believe musicians do. This line of thought led me to challenging questions. When in music do we negotiate with one another? Construct and share meanings relevant to the problemsolving task at hand? Participate in coordinated, synchronous activity? Pursue a continued attempt to construct and maintain a shared, new, musical concept? In the world of chamber music, and the string quartet, I found evidence of collaboration. Consider these quotes from Blum's (1986) book of interviews with the renowned and longestablished Guarneri Quartet.

In what seems a perfect description of shared meaning, cellist David Soyer describes the nature of his group's collaboration:

Our way of ensemble playing is not that someone leads and everyone else just follows...but in fact, everyone feels it at the same time; everyone is thinking toward a central point: the start of a piece, a ritardando, or whatever it may be. We don't follow each other; we play together. (Blum, 1986, p.15)

Regarding the negotiation inherent in collaboration, violinist Arnold Steinhart says, "There's a constant working-out process... tomorrow we'll disagree in a slightly different way from today, so an evolution is always taking place" (p. 7).

Evidence of coordinated, synchronous activity is found in the words of violinist John Dalley: "I often have to read Arnold's mind to make sure that we'll all be together...should Arnold play his melody slightly faster or slower, we'll adjust right away" (p. 130). And violist Michael Tree seems to speak directly to the quartet's 
continued attempt to construct and maintain a shared musical concept when he says, "Should I find myself committed to a bowing that [Dalley] isn't doing at the moment, it's my job to make my phrasing sound similar to his, even if we aren't actually bowing in the same manner" (p. 6).

As the preceding quotes from the Guarneri String Quartet indicate, musical contexts are well-suited to illustrate the benefits of collaboration for learners. The CTSG teachers believe their student musicians should be able to feel the convergence and synergy in a similar and developmentally appropriate way to what is described by the quartet members above. Recent music education research has documented the value of group collaboration in helping students solve musical problems or acquire deep understanding of concepts. Wiggins (2000) credited collaborative problem solving in group improvisation and composition with creating a strong shared understanding of the musical concepts involved. She found this resulted in empowered musical thinking by the group as a whole as well as by individuals. Younker and Burnard (2004) have analyzed small group interaction as an integral part of compositional collaboration, as students identify problems, generate solutions, and evaluate their success. Numerous other studies, discussed in detail in Chapter Two, have focused on the strength of collaborative processes in stimulating creative and critical thinking through the process of group compositional problem-solving (e.g., DeLorenzo, 1989; Dillon, 2003; Wiggins, 2000; Younker \& Burnard, 2004, 2008).

Studies of student-directed collaboration in non-compositional musical contexts are fewer, but extant research is positive on the value of constructing space for these sorts of activities to occur within the arena of music education. Berg (1997) found that 
members of coached high school chamber ensembles "challenged each other to work at a higher developmental level by requiring peers to clarify, elaborate on, or justify a problem solution" (Berg, 1997, p. iv). Davidson and Good (2002) studied the way social, cultural, and emotional factors contributed to a college quartet's preparation for a performance. The quartet's interactions focused on solving musical problems such as rhythmic control and tuning, and grappling with performance anxiety and group conflict.

Researchers have alluded to the positive benefits of student musical collaboration. King $(2004,2006)$ drew together a framework for ensemble rehearsals containing collaboration as a central component. She cites the possibility for collaborating performers to take leadership and negotiate their own technical and interpretative musical ideas. Hoffman (1991) was positive on the aspects of collaboration in a computer-aided music theory class, citing evidence of ongoing and shared understanding in a lively atmosphere that contrasted with the passive attitudes of non-collaborating students.

In introducing a symposium on "Musical Collaboration" at the Eighth International Conference on Music Perception and Cognition, MacDonald \& Miell (2004) summarized the fairly small amount of literature on musical collaboration. They cited a lack of analysis of the social interaction that occurs when people collaborate in musical activity, calling this a "fruitful domain" for further research. A subset of this missing research is investigations of elementary school student musical collaboration, especially in musical contexts unrelated to student composition. This study provides information on the CTSG teachers' perceptions of student collaboration in diverse activities: playing instruments, making arrangements, improvising in groups, composing, and singing. 


\section{Conclusion}

After investigating the extant research in both student and teacher environments, I still had many questions about the nature of teaching and learning in collaboration. My need to explore both student and teacher collaboration led me to construct this study with a dual purpose. The main focus of this study, and the basis for my first two research questions (How do the participants describe their experience in the CTSG, and how has the focus on collaboration in the CTSG changed the teaching practice of these teachers?) was to examine CTSG as a professional development structure. First, I sought to understand how the participants described their experience in the CTSG. I also wanted to know how the focus on collaboration in the CTSG changed the teaching practice of these teachers. I wanted to know if the CTSG would feel like a community to us. I wanted to discover how the CTSG worked, from all perspectives including my own.

But the secondary focus of this study went beyond this, and led me to ask my third research question (What can these music teachers tell other music educators about collaboration?) I wanted to know not just how the CTSG worked for the participants, but what the CTSG participants learned about student collaboration during our seven weeks of meetings. I needed to find out what the CTSG music teachers could tell other music educators about collaboration in the elementary classroom.

The following review of the literature on collaboration in educational settings establishes a context upon which both functions of this study are based. I begin by discussing scholarship and research on groups of teacher learners, first in general education and then in music education. I delineate the characteristics of communities that 
have been found important to professional development settings. Second, I discuss the important points from literature on student collaboration, in general classroom and then music classroom settings, including student chamber music. 


\section{Chapter 2}

\section{A COMPILATION: REVIEW OF THE LITERATURE}

This compilation ties together four bodies of literature: (a) research and scholarship in teacher communities outside of music education; (b) research in music teacher communities; (c) research on student collaboration in subject areas other than music; and (d) research in music student collaboration. I begin by presenting an overview of recent writing on the role of community in professional development. Scholars have considered both the benefits and the uncertainties that surround the use of teacher community as a professional development structure, and some of those views are briefly presented here.

Next, four substantial studies of teacher community are reviewed (Grossman, Wineburg, and Woolworth, 2000; Horn, 2005; Little, 2003; Rousseau, 2004). From those researchers' conclusions I identified five major factors that seem to positively or negatively affect the effectiveness of teacher community in professional development: (a) the length of commitment to the community; (b) the tension between the goals of improving content area knowledge and pedagogical skills; (c) the way teachers with varied goals for development participated, and in different roles; (d) the avoidance of or enabling of honest examination of teaching practice; and (e) the ways teachers brought issues forward for discussion. I then examine four studies of music teacher communities (Dolloff, 1996; Junda, 1994; Robbins, 1995; Roulston, Legette, DeLoach, Buckhalter- 
Pittman, Cory, \& Grenier, 2005) for the presence or lack of the same five factors. A concluding section pulls these bodies of literature together in terms of common characteristics of successful professional development communities.

I then turn to an examination of the processes of group interaction and how these processes may relate to student learning. This portion of the review details literature regarding collaboration in general educational and classroom settings (Azmitia \& Perlmutter, 1989; Barron, 2000; Chinn, O'Donnell, \& Jinks, 2000; Fischer \& Mandl, 2005; Forman, Stein, Brown, \& Larreamendy-Joerns, 1995; Kovalainen and Kumpulainen, 2007; Kynigos \& Theodossopoulou, 2001; Palincsar, 1998; Palincsar \& Herrenkohl, 2002; Perret-Clermont, Perret, \& Bell, 1991; Roschelle, 1992; Roschelle \& Teasley, 1995; van Boxtel, van der Linden \& Kanselaar, 2000). These studies define collaboration and codify what variables may affect the success of collaborating groups.

Finally I examine the literature on student collaboration in music education (Burnard, 2002; DeLorenzo, 1989; Dillon, 2003; St. John, 2006; Wiggins, 2000; Younker \& Burnard, 2004, 2008) and in student chamber music groups (Berg, 1997; Davidson \& Good, 2002; King, 2006). Perspectives on Teacher Communities

The last 20 years have seen an increase of teacher groups that attempt to provide a basis for shared, reflective inquiry around problems and issues of teaching. Site-based or local teacher work groups, inquiry groups, and communities of practice are becoming more common. This section of the paper is a discussion of the recent perspectives on collaboration within teacher communities and professional development. 
Little (1993) cites "intellectual, motivational, and attitudinal" benefits to teachers who participate in what she calls "pragmatically messier" (p. 142) alternatives to the common professional development workshop model. A community-based, collaborative model is a messier alternative; it is not based on expert-led seminars or workshops but rather, on teachers sharing knowledge and working together to grow professionally. It offers substantial learning opportunities for teachers who may discuss and debate critical issues in schools. The unscripted nature of these conversations stands in relief to the traditional clinic or workshop that is a one-way delivery of tips, tricks, or ideas. Conversations within teacher groups, or communities, may be the place for educators to consider what Feiman-Nemser (2001) would call the hard questions, the disagreements that are opportunities to consider and clarify beliefs:

The kind of conversation that promotes teacher learning differs from usual modes of teacher talk which feature personal anecdotes and opinions and are governed by norms of politeness and consensus. Professional discourse involves rich descriptions of practice, attention to evidence, examination of alternative interpretations, and possibilities. (p. 1043)

But not all teacher groups would function in the way Feiman-Nemser suggests; they may work at concurring on easy answers rather than asking hard questions. The ways in which teachers handle disagreement, and the way interactions within the community are designed, are vital considerations (Curry, 2008).

All communities are not created equal. Wenger's (1998) book Communities of practice: Learning, meaning and identity is a work that theoretically examines how people learn from and with others. For Wenger, a "community of practice" is a network of people where collective learning takes place: "Communities of practice are groups of people who share a concern or a passion for doing something and learn how to do it as 
they interact regularly" (retrieved from www.ewenger.com, October 15, 2008).

According to Wenger, the purpose, setting, and social aspects of group interaction affect learning. The idea of a community-of-practice comprised of teachers has been a popular consideration for professional development.

But if the goal of professional development is transformation of teacher knowledge and improved student achievement, there is little consensus on the relationship between a strong teacher community and achieving that goal. A community of teachers may work together to re-invent teaching practice, or may collaborate to reinforce the status quo (Rousseau, 2004). McLaughlin and Talbert (2001) label groups with a strongly shared interest in maintaining tradition, or agreed-upon norms "strong traditional communities." These groups have an uncommon unity in their shared beliefs. They call groups who work to reform teaching "strong teacher learning communities," which are even less common.

It is also rare to find teacher communities that persevere in a pursuit of dialogue beyond the superficial. In a review of the formal professional development literature preceding her study of daily teacher interaction, Little (2003) summarizes:

Substantively, these studies [of explicit, formal professional development] point to the difficulties teachers encounter in achieving sustained and deep consideration of teaching problems and possibilities, even in conditions formally structured for that purpose, and to related difficulties in contending with difference and disagreement on matters of practice. (p. 919)

Equating community formation with learning may be problematic. One concern about forming a tightly knit professional community is that we might replace "the isolated classroom teacher with the isolated teacher group and balkanized workplace" (Little, 2003, p. 939). Some community values seem positive on the surface, for example 
sharing knowledge, refining knowledge based on shared beliefs, enculturating novices into a professional community. However Cousin and Deepwell (2005) refer to the "more sinister dimensions of community" (p. 58): the tendency to minimize or be intolerant of conflict, mandatory obedience to group norms, exclusionary actions, and control of who may join a community, possibly based on sexism or racism. Noddings (1996) refers to this as the "dark side" of community: its "tendencies toward parochialism, conformity, exclusion, assimilation, distrust (or hatred) of outsiders, and coercion" (p. 258). As she sees it, a community can just as easily support movements toward uniformity or mediocrity as it can support actions celebrating difference.

Another danger of a community of practice is that learners may "arrive at a pedagogic setting with congealed practices from another kind of setting" (Cousin \& Deepwell, 2005, p. 61). Or, a community of teachers may come together and learn about teaching, but be unable to make changes to the status quo back at their own schools.

Not all communities result in learning, and not all groups are communities, no matter how supportive or friendly. Grossman, Wineburg, and Woolworth (2000) state that for a group to be a true teacher community of professional development value, its central purposes must be the development and maintenance of a student-centered view, paired with opportunities for teachers' "continued intellectual development in the subject matter" (p. 14).

Researchers have recently begun to examine whether a "community of practice" is actually as effective as the term sounds. Brown and Duguid (1996) point out the "connotation of warm persuasiveness" (p. 53) that surrounds the term "community of practice." They caution us to look to the original source, Wenger (1998), for a non- 
idealistic look. Wenger states, "because the term community is usually a very positive one, I cannot emphasize enough that these interrelations arise out of engagement in practice and not out of an idealized view of what a community should be like" (p. 76-77, italics added). In fact, "community of practice," while a useful lens or metaphor by which to examine local teaching practices and teacher interactions, has also been found by researchers to be an unsuccessful professional development design professional development (Yamagata-Lynch, 2001; Palincsar, et al., 1998).

Little (2003) likewise questions the automatic assumption that participation in communities will improve teacher attitude or skills. She calls the resources for teacher learning and innovation within teachers' professional communities a black box on which relatively little research exists:

Claims about the generative power of professional community for individual development and for whole-school reform frequently founder on evidence that not much has changed at the level of teaching and learning in the classroom. (Little, 2003, p. 940)

\section{Issues in Teacher Professional Development Communities}

There is much we in music education can learn through an examination of the literature on teacher professional development communities within general education (Grossman, Wineburg, \& Woolworth, 2000; Horn, 2005; Little, 2003; Rousseau, 2004). What are the hallmarks of communities successful in supporting teacher learning? What elements have contributed to the failure-to-thrive, or lack of teacher learning, in some communities? What factors enable teachers to have honest conversations about teaching practice? These four studies are grouped for discussion here according to three broad elemental categories in the teacher communities studied: the long-term commitment to the community, teachers' ability to take on various roles, and the way teachers brought up 
topics to talk about.

Long-term commitment, diverse teacher goals and roles. Grossman, Wineburg, and Woolworth (2000) performed an 18-month study (part of a three-year project) of a professional development community of 15 English and history teachers in a Seattle high school. The researchers intentionally set the purpose of the community as two-fold: construction of a new interdisciplinary curriculum, and to inspire teacher learning in their subject area. To accomplish these purposes, the teachers were to meet monthly for a fullday book club discussion, and to plan a humanities curriculum. The teachers were all volunteers, but the convenient onsite location, stipend, and recommendations by administrators that certain teachers join the group, attracted a different group than those who typically sought out professional development in that school. The researchers collected field notes, emails, journals, and did interviews with the participants, but most of the analysis regarding the process of community formation was based on data from their monthly meeting transcripts.

Within the community there were tensions between teachers seeking "direct applicability in the classroom" and those seeking "the more distant goal of intellectual renewal" (Grossman et al., 2000, p. 14). The researchers describe difficulty in establishing community in this professional development setting. For example, the researchers observed pseudocommunity, or pretending to have a real community before one actually existed. A particular difficulty was the unwillingness of teachers to share video of their own teaching; the researchers acknowledge that the teachers felt this posed a threat to their privacy, and in this context would probably not portray authentically the teachers' true practice. Tensions in communication and warring factions marked the 
meetings.

Despite the difficulties, which they attribute in part to placing teachers in an unfamiliar situation with uncertain norms, the researchers found the professional development was successful in renewing teachers' intellectual curiosity. The teachers learned new content, and changes in the discourse show a greater appreciation for their colleagues' actions. Two beliefs seemed to develop: that colleagues can be great resources for learning, and that teachers are responsible first for student learning and second to each other. The project design contributed to these positive results. The researchers refused to permanently take the role of university experts, or group leaders. This allowed teachers to participate along "multiple corridors" (p. 15): leaders, followers, curriculum developers, readers. Thus, although the community spent a year trying to bridge these different expectations, the researchers tell a story of uneven but steady progress toward real community. Group members were empowered to take on responsibility for negotiating norms of social engagement, norms that allowed teachers to become honest about their beliefs and practice:

Learning to argue productively about ideas that cut to the core of personal and professional identity involves the skillful orchestration of multiple social and intellectual capacities. While this vision of community may seem utopian, we believe that it is exactly the kind of work that teachers require of students if classrooms are to become communities of learners. (Grossman et al., 2000, p. 38)

This eighteen-month study was a part of a three-year effort by the researchers to build a professional development community in this school; the researchers state the length of this project was largely responsible for its success. "If other projects that seek to create community in the workplace do not encounter the obstacles we describe, we suspect they either began with motivated, self-selected volunteers... or met for only a 
limited amount of time" (p. 48).

Avoiding or confronting discrepant beliefs: teacher talk matters. Rousseau (2004) studied a community of five high school pre-Algebra teachers for one year in order to better understand the creation and functioning of professional community. The teachers in the study were all participating in a national professional development course for Algebra and pre-Algebra teachers. In order to determine what beliefs the group shared, she observed their classes, interviewed each teacher, and observed and tape-recorded weekly meetings.

Rousseau found that the group failed to implement or sustain the reform model of teaching, Mathematics in Context (MiC), a curriculum chosen by the teachers at the beginning of the year as a way to help their students learn to engage in mathematical problem solving. Rousseau cites conflict between "internal, but shared, inconsistencies" (p. 793) between the group's shared values as partially responsible for the group's inability to sustain reform. The group shared two visions simultaneously: a belief in rulebased, drill-and-practice teaching and a more open-ended questioning, reasoning-oriented way of looking at pre-Algebra. The group also "characterized their students as barriers to reform" (p. 793). Although these teachers seemed to want to change their practice, they avoided conflict by never looking at the discrepancies or inconsistencies in their group beliefs: they never examined what "they, as teachers, were doing, and their own roles in the implementation" (p. 794). Throughout the year many of the teachers abandoned MiC in favor of worksheets and more skill-based, traditional practice, responding to the sporadic improvement in student problem-solving and what they saw as variable amounts of success in students' ability to do the class reading and homework. By March MiC was 
almost completely gone. Rousseau found that by placing the blame on students and asserting their beliefs in the benefit of skill-based practice worksheets, the teachers avoided confronting the real conflict: "between their vision of what they should be doing and the reality of what they actually were able to accomplish with respect to reform" (p. 794).

Rousseau recommends that facilitators of professional development communities concentrate on addressing the relationship between the teachers' views on teaching and learning, and their beliefs about their students. Also, she recommends that professional development should enable confrontation, rather than avoidance, of inconsistencies in teacher beliefs. In this case, a failure to confront the discrepancy between the ideal vision (a smooth implementation of a reform curriculum) and the reality (the teachers' lack of ability to implement reform) gave the teachers a way out without having to do any real self-reflection. Rousseau argues that professional development should have a mechanism for helping teachers recognize and confront avoidance tactics.

Horn (2005) identifies such mechanisms in her study of math teacher community in two high schools. In a comparative case study of math teacher professional development at two high schools ("East" and "South"), Horn found that specific types of conversation and interactions greatly contribute to teachers' ability to have honest, accurate conversations. She conducted structured and ethnographic interviews, made audio and video recordings of formal and informal teacher activities, observed teachers in their classrooms, and studied school and classroom artifacts in order to understand how teachers' interactions related to their learning about practice. 
South High had a well-structured reform network in place, featuring paid professional development for teachers, teacher research groups, and project based learning. At East High, only certain departments were reform-oriented, and efforts varied widely across the school. The math department was one of the most progressive and had a long history of working together on curriculum and teaching. The department was able to do its own hiring and tended to select teachers who would fit into the model of committed, questioning educator.

Horn developed a unit of analysis called "episodes of pedagogical reasoning (EPRs)" (p. 215). These were units of teacher talk in which teachers made explicit some type of reasoning about their practice. In an EPR, teachers would describe a situation or ask a question about teaching, followed by some sort of explanation or elaboration. EPRs were coded, and EPRs on topics of "student engagement," "student failure," "assessment," "alignment of students and curriculum" were frequent. The EPRs were used to compare the way the teachers at each school "publicly and collectively reasoned about their work" (p. 215).

The EPRs at South High were consistently examples of teacher talk that featured details about the teacher-student relationship, student motivation, student classification. Teachers were committed to their students as people, but not necessarily as math learners. Teachers at South did not often have realistic conversations about the school reform efforts: "their monthly mathematics meetings were taken over by bureaucratic demands...limiting opportunities to examine mathematics teaching and learning” (p. 218). Because the department only met monthly, their meetings were mostly logistical and not teaching-oriented. Most of the EPRs occurred in the hall, quickly, and informally. 
The larger, school-wide reform effort in place faltered in the math department; the teachers were unable to work together to figure out math's place in the school's focus on "less is more," or in other words, mastery, not coverage of content.

The reform effort at East High was subject-specific and centered around using "group-worthy problems" (p. 219) in the math curriculum: problems that were important, include multiple tasks and draw on collective efforts in the class, and have multiple representations and solutions. Because the teachers met at least weekly, they were tightknit and as a community they communicated regularly in other ways about teaching (email, hall conversations, in the workroom). Their communications were specific, and gave explicit information to their colleagues about teaching and learning. Horn reported difficulty in even creating two comparable case studies, as the density and complexity of teacher interaction at East was so much richer.

Horn found evidence at both schools for the use of different kinds of Wenger's reifications: artifacts like slogans, curricular materials, and assessments that became shared points of reference for the teachers. Teachers at both schools developed community meanings for their system of classification (of students, curricula, teachers), and teachers at both schools engaged in collegial conversations of "teaching replays" ( $p$. $225)$ to reenact and explain interactions with students.

The categories for talking about students at South High were limited and static. Horn found that teachers labeled students as fast, slow, and lazy. At East High the math department leadership promoted a subtle, detailed category system, and insisted on talking about student achievement in a less fixed way, with more attention paid to the ongoing quality of learning opportunities. Teaching replays at South focused on 
characteristics of student personalities, behavior, and motivation. Teaching replays at

East focused on the nature of the "group-worthy problems" that were a constant, relevant topic of discussion among the teachers.

Horn found evidence of "transformative learning" at East High: "learning that challenged existing assumptions and had the ability to change classroom practices more fundamentally" (Horn, 2005, p. 217). Teachers at East High showed evidence of transformative learning in the way their community supported changing their habits of mind and point of view: key concepts in transformative learning theory (Mezirow, 2000):

The East math teachers developed work and interaction practices that supported new habits of mind for teaching and provided ways for them to reflect on their points of view. By coordinating their understanding of their reform slogans, questioning the assumptions underlying their categories for students, and rendering classroom practice in their conversations, the East math teachers made many aspects of teaching and learning available for collective reflection and inquiry. (Horn, 2005, p. 231)

South High was less successful at changing teachers' points of view. Horn attributes this to the teachers' few opportunities to translate reform into actual classroom practice: "there were no mechanisms within the reform for questioning assumptions about students and math learning or opening up windows on classroom practice" (p. 231).

Horn makes two specific recommendations for teacher professional development in communities: (a) the development of carefully nuanced language for shared artifacts and category systems, which help teachers discuss and reason more precisely about problems of pedagogy (i.e., going beyond traditional ability-centered language_- "fast" and "slow" - to characterize students); and (b) using collegial conversations - teaching 
replays and rehearsals — as a kind of stage on which to apply and look at values and principles.

Had Rousseau's (2004) teachers, or the South High teachers, been able to make their teaching practice visible to their colleagues, and been able to find equally productive ways to talk about and analyze exactly what was going on with the students in their classrooms, it is possible they would not have retreated so helplessly from their reform effort. It is clear from these two studies that teachers need support in ways to talk about their actual classroom events.

Coming together around reform efforts is also essential. As Horn says, this is enhanced when teachers can work together to make meaning of reform in their unique context: "teachers' understandings may be well coordinated when they have multiple and ongoing occasions to reflect on their meanings collectively" (p. 229).

Because Little (2003) questioned "the premise of improvement in teaching knowledge, practice, and commitments associated with participation in professional communities" (p. 916), she set out to analyze the teacher learning that occurred within three teacher-led groups that were considered collaborative and innovative. This was an intensive case study of three episodes of routine, outside-of-class interaction of three different groups of math and English teachers in two high schools. She collected data in the form of observations, interviews, school documents, and audio- and videotaped recordings of teacher interactions.

Little found that teachers' decontextualized portrayals of classroom happenings make up a large and meaningful part of teacher talk, and supply material for the group's 
work, despite the fact that no one can ever be completely sure of what is meant in these short-hand, "discrete, condensed, and desituated" (p. 936) accounts.

Little identified three aspects of teacher talk that complicate their learning from each other: (a) teachers' accounts of practice are never as clear as lived or observed practice; (b) classroom accounts are often brought up in response to some topic at hand, and not necessarily to illustrate or interpret a topic of importance to the major enterprises of the group, making it hard to say "whose representations matter, and how?" (p. 937); and (c) accounts of practice are time-condensed, ephemeral, and fleeting in the ongoing talk of teachers.

Little concludes that the information (talk and artifacts) teachers bring forth for other teachers to examine varies widely from group to group, although each group professes goals of student success, innovation, and collaboration. Each account of teacher talk might open up some opportunities for teacher learning, and close others. Teachers also co-constructed representations of practice, by interrupting or changing descriptions being made by a colleague. Only certain resources are brought forward at certain times to certain members of the group. Little states that studying this path of language coconstruction can show a great deal about the patterns and norms of participation and dialogue within the group.

Little found that in these groups, "teacher learning seems both enabled and constrained by the ways that the teachers go about their work...the impulse to question practice resonates against the press simply to get on with it" (p. 940). Understanding this play between tradition and innovation, and helping teachers talk about it, is key to structuring productive professional development. Little, like Horn and Rousseau, also 
points out the importance of clarity in teacher conversation within professional development communities.

From the above studies, I have distilled five major factors that impacted teacher community: (a) the length of commitment to the community; (b) the tension between the goals of improving content area knowledge and pedagogical skills; (c) the way teachers with varied goals for development participated, and in different roles; (d) the avoidance of or enabling of honest examination of teaching practice; and (e) the ways teachers brought issues forward for discussion. The next section of this chapter examines research on several music teacher communities for the presence or lack of the above five factors. Issues in Music Teacher Professional Development Communities

An examination of the literature on music teacher communities confirms the influence of some of the same factors in the studies above. The length of commitment to the community and the how practice is represented seem to be influential to some degree in the research on music teacher communities. However, the professional development within the musical communities seems more reliant on university or expert leadership. While Grossman, Wineburg, and Woolworth's (2000) productive tension between goals - deepening of content knowledge or improvement of pedagogical skills — is present in these communities, the productive tension between teachers as they begin to take on different roles in group leadership is not evident. Also missing is a detailed look at how music teachers bring issues forward for collective viewing and discussion: we don't know if and how music teachers might place their practice above Little's (2003) “group horizon of observation" (p. 938). 
Junda (1994) studied a group of K-3 general music teachers $(N=12)$ in New Jersey who participated in a year-long professional development program called "Collaborative Approach to Music Instruction.” The program was intended to improve the musical and instructional skills of the teachers, and in turn the musical skill of their students. Teachers enrolled in a graduate-level Kodàly music education course designed to help teachers develop instructional strategies and identify appropriate repertoire. A strong focus of the class was improved teacher musicianship. Teachers were guided in creating strategies for the implementation of Kodàly method in the classroom, and were responsible for trying out (and documenting on video) the new content and procedures in their classrooms. The course instructor used a clinical supervision model in visiting each of the twelve classrooms five times in the year to give specific recommendations and feedback.

Formative evaluation in between semesters indicated that the teachers needed further review of teaching sequence, assessment, and lesson planning, all of which were reinforced in the second semester coursework. The summative evaluation of the program involved collecting data on teachers' musical and instructional skills, attitudes, and on their students' musical skills.

Junda found the teachers' musical skills were weak. Their musical skills and their knowledge of Kodàly pedagogy improved through this course. This confirms Grossman, et al.'s (2000) contention that communities should be structured to involve a productive tension between development of content area skills and teaching skills. Student data revealed improved sight-reading skills, and a larger repertoire of songs, lending support to the idea that this professional development was successful in terms of student learning. 
The teachers in the program believed that the supervisory observations were one of the most important factors in achieving these results, because the instructor's feedback helped them develop their instructional skills in ways particularly appropriate to their jobsites and assignments.

The video documentation and classroom observations were a transparent way of establishing and communicating shared values of practice within this community. Unlike the general teacher education communities above, by making video and visits part of the development, this group was able to formally represent their teaching practice in an objective way.

The Kodàly teaching sequence, especially the sight-reading curriculum, was important in this community as the teachers worked with the instructor throughout the year to improve and to document their acquisition of strategies to develop readingreadiness and sight-reading. At the end of the year, the teachers stated in written evaluations and exit interviews that participation in the program had a positive effect on their instructional skills.

The program director acknowledges that the Kodàly method curriculum guides outline a specific instructional sequence; the participating teachers did not have to decide what to teach, but were able to concentrate on how to teach designated concepts in a prearranged order. This is different than a teacher community that works to create its own curriculum (e.g., Grossman, et al., 2000); we have no information on whether teachers had to confront their own beliefs and argue with others regarding Kodàly methodology.

Post-program evaluations affirm the positive effects of community and the presence of strong, shared values regarding pedagogical content: "They [teachers] 
realized that their colleagues were a valuable resource, and they enjoyed sharing new and innovative methods of teaching the same material" (p. 15). We do not know if the reliance on university instructor and established methodology had any effect on teachers' ability to rise up to take roles of leadership and really own their personal development and transformative change, as seemed to happen in the Grossman et al. and Horn studies discussed above.

Dolloff (1996) conducted research on an inservice choral teacher development program designed to promote and develop expertise in choral music education. Drawing on research on acquisition of expertise in other domains, Dolloff hypothesized that the “apprentice/role-model format" (p. 4) would be a crucial part of developing choral music education expertise, and that the expert-mentor plays an important role in teaching teachers.

Continuing development of expertise was the goal in this performance-based development project. Twenty teachers completed a three-year, three-phase project which used a cycle of practice and expert coaching with the master teacher, albeit in large groups. The teachers learned the music themselves in a seminar which served both to immerse the teachers in music performance and give them a students'-eye-view of a pedagogical model. Next, the master teacher taught the students of the participating teachers the music in a one hour intensive rehearsals, 100 children at a time. Teachers then worked with their own students for a period of time, refining the music (the teacher implementation phase). They would return for another session with the master teacher for help with any questions of technique or practice that arose during teacher implementation phase. The master teacher then led the children (in groups of 200) in another intensive 
rehearsal. This cycle of teacher seminar-rehearsal/demonstration continued, leading to a culminating concert. Dolloff collected teacher questionnaires, video documentation, and field note data and documented a perceived increase in teachers' capabilities and greater self-esteem.

Dolloff credited the success of this program to three factors: an extremely strong and world-renowned master teacher/conductor who was able to model successful rehearsal strategies with the teachers' own choirs, the alternation of expert modeling with teacher practice, and the three year commitment to professional development made by the participants.

In this professional development program Dolloff studied, the master teacher espoused the praxial view of music education, or music education through music performance (versus music education through aesthetic perception).

This philosophical difference leads to a pedagogical difference, requiring of the teacher a different set of teaching skills and dispositions... in any study of teacher education it is important to know the philosophical foundation that underpins the teaching in the domain in order to define what constitutes successful teaching in that domain. (Dolloff, 1996, p. 6)

In this community of practice, teachers sought to improve conducting and rehearsal expertise according to a specific, praxis-based model of music education. The norms of good teaching were established and evaluated according to this model, shared by the master teacher, the researcher, and the program director. No evidence in Dolloff's analysis points to any teacher disillusionment with this teaching style; rather, they tried hard to approximate the modeled teaching in their own classrooms. It may be that elementary and middle school choir directors on the whole have a leaning toward a performance-based praxial philosophy of music education; the group of teachers in this 
study may have previously espoused this style of teaching and were not enculturated into it solely during the course of the professional development.

The involvement of this number of teachers would seem to indicate that a large variety of experience and background was represented, and that teachers participated differently according to where they were on their career trajectory. We have little information on the norms of community, or the teacher interaction, that may have developed in the course of these three years. What we do know is that once again the music teachers worked to establish within their own classrooms a curriculum modeled by an expert, and that the length of the development was effective.

Robbins (1995) studied the informal talk and group inquiry of a group of six teachers who completed two summers of Orff-Schulwerk teacher training courses together. During the first year the six teachers kept journals and met four times to discuss their work. In the second year teachers were asked to act as teacher-researchers, with a focus on questioning "those aspects of their teaching that continued to cause uncertainty or create some tension between the training and practice" (p. 50).

Some of the questions concerned "dissonance" (p. 50) between the teaching model presented during the summer and the incompatible scheduling and facilities at the teachers' home sites. Similar questions arose regarding the organization and translation of the Orff repertoire from the training sessions into previous curricular formats. The participants had questions about pedagogy, as in which lessons from the summer should be used in certain situations and with certain groups of students. Not all the ideas were able to translate successfully to the classroom, at least not immediately or easily. For 
example applying some of the Orff principles of student improvisation and creative spontaneity resulted in "bumpy lessons" and "creative fallout" (p. 51):

For some [teachers], the fear of losing control was overshadowing their intention to try new things. Together they sorted out the importance of their risk-taking, using their growing understanding of Orff-Schulwerk as a check against what was happening in their classrooms. (p. 51)

It is not clear if the teachers modified their ideas of Orff practice, became more successful in their implementation, or both; but Robbins states at the end of year one, the questions became "less about how to do something and more about what it means to use an Orff approach" (p. 51). Implicit in that statement is the idea that the teachers switched from thinking about techniques and tips to considering broader philosophical ideas behind the methodology.

In year two of the study the participants seem to have more strongly identified themselves as Orff teachers. "The teachers were ready to have their voices heard within the larger community of Orff-Schulwerk experts. No longer isolated from other teachers, participants in the Orff SPIEL cooperative realized the power of the professional community that they had built" (p. 51). Just as the teachers in the Grossman, et al. (2000) worked through stages of lessening their need for privacy, and gradually opening up the private realms of their individual classroom beliefs, the teachers in this study eventually drew strength from the community to inform their classroom practice.

The work of the Orff SPIEL group confirms Grossman, et al.'s contention that a supportive collective serves as a training ground in which teachers try out new ways of talking, thinking, and knowing; all of which they can take back to their own milieu. Teachers who recognize and understand differences between the voices of their colleagues are more likely to recognize and understand student needs (Grossman, et al., 
2000, p. 33). Robbins felt the Orff SPIEL teachers changed; they began to be teachers who worked to establish an atmosphere of student-teacher mutuality, not just in their own classrooms but in the summer sessions as well when they were the students.

Robbins emphasizes the way the teacher participants learned to enjoy questioning, and to participate more fully in the construction of teaching knowledge. She offered space for teachers to combat isolation through community. The findings from the study primarily related to translation of the Orff repertoire from the training sessions into specific local contexts. The participants had questions about pedagogy, as in which lessons from the summer should be used in certain situations and with certain groups of students, how the lessons could be scheduled, and eventually, more philosophical questions. "What is flow and fluidity in Orff lessons? What happens when students are encouraged to be spontaneous in music?" (p. 51). To answer these questions, the teachers intentionally and meaningfully scrutinized their lessons, and shared previously "private worlds" through video. Robbins found the teachers became more thoughtful and purposeful about their teaching.

In the course of study the teacher-researchers seem to have more strongly identified themselves as teacher-leaders who now have a stake in and a way to contribute to a more formal body of teacher knowledge. Robbins concludes her analysis by emphasizing the empowerment of the teacher-researchers, and reminding us that teacher research is about the power: "who gets to participate in the creation of knowledge about teaching and learning” (p. 52).

Roulston, et al. (2005) were involved in a music teacher-research community designed to contribute to the professional development of early-career elementary music 
teachers. They wanted to investigate how a teacher-research community, involving both university educators and teachers, could be structured around a "practice-based orientation to research" (p. 4) in which group members would design and conduct individual research projects. The group consisted of two university educators, and second- and third-year elementary music teachers.

One teacher in the research group elected to study boys' participation in elementary chorus; the other investigated how best to work with students with learning disabilities in music class. The group met monthly for three hours to discuss action research models and methods. As the classroom teachers created research questions and collected/analyzed data, the university educators served as research mentors by providing guidance in such areas as locating literature, creating research instruments, and gaining university and district study approval.

The group performed a group self-study by examining "naturally occurring data" (Roulston, et al., 2005, p. 7) related to the experience of being in the research community. All meetings were audiotaped and transcribed, the four group members interviewed one another, "wrote early and often" (p. 8) and shared the writing, so that all group members could look at primary data, read, revise, and check interpretations of the group experience.

Roulston, et al. found that one outcome of participation in this teacher-research community was the eventual development of a team that provided a supportive space in which to try new ideas and ask questions. Analysis of group discussion indicated the elementary teachers learned new ways to consider and reflect on their practice; for example, one teacher was able to analyze group interactions in her classroom based on 
data collected by a paraprofessional, finding that what was really going on in the classroom was different from her prior perceptions.

The collaboration within the group helped to combat the feelings of isolation common in music teachers and university faculty. Faculty members found that working with teachers on research informed their own practice as teacher educators. While the university educators began the project acting as organizer and leader, the roles blurred as the group members shared responsibilities related to writing about and presenting their work at conferences. The elementary teachers were socialized into the role of "researcher"- at the same time, they strengthened their identities as teaching professionals and received mentoring support from the faculty members. The group concludes that "teacher research collaborations between university educators and practicing teachers can supplement existing mentoring programs and contribute to the development of... professional learning communities" (Roulston, et al., p. 17).

This study seems to incorporate all five of the factors identified above that may contribute to teacher professional development in a community: length of commitment to the process (one year), diverse teacher goals and roles (action research, curriculum development, leadership in research, collaboration with university faculty), honest teacher talk (aided by the focus on gathering data and the self-study, making it less subjective), and finally, the ability of all members to bring issues forward relating to the topic at hand through process of writing, interpreting, and revising as a group.

\section{Characteristics of Successful Professional Development Communities}

In looking at the preceding research on teacher learning in groups for evidence of the "generative power" (Little, 2003, p. 940) of community, several broad themes emerge 
characterizing successful professional development, defined as transformation of teacher knowledge, or some sort of fundamental shift in thinking (Feiman-Nemser, 2001; McLaughlin \& Talbert, 2006). Fundamental aspects of Wenger's community of practice concept are evident as well.

Wenger's mutuality, competence, and imagination. Groups were successful when they involved people at various levels of competence and experience, and when productive conflict occurred at these boundaries. Imagination, according to Wenger (1998), allows learners to adopt perspectives from across boundaries, and mutuality allows group members to participate meaningfully wherever they are on their career trajectory. One person was not seen as the sole authority or the most competent, which allowed peer learning to flourish.

Wenger's shared repertoire, practice, and alignment. Three of the music teaching communities described herein were all successful teacher learning groups that shared particular views on teaching practice - Kodàly, Praxialism, and Orff-Schulwerk. Knowing how to teach involved a competence in certain ways of teaching that are typical to and shared by the group: their identity. "Knowing how" to teach in these sorts of communities is communicated by experts, and developed by novices as they grow into more competent members of the community. This creates strong shared repertoire, identity, and goals: consistent with Wenger's basic characteristics of a community-ofpractice. Alignment, or the practical underpinnings of a group, allowed these groups to flourish, as they were organized with specific goals in mind (e.g., enrollment in Orff levels or a graduate-level Kodàly course; preparing for a culminating concert with a master conductor). 
Wenger acknowledges a tension group's need to be led, and the need for individuals to enjoy mutuality of engagement. The back-and-forth of peer learning may be a source of problems or solutions. Creativity may be squashed in a conformist community, or the strongly shared values may be a source of insight. This reality needs to be acknowledged when using an expert modeler, or well-established methodology, to provide professional development. "The very characteristics that make communities of practice a good fit for stewarding knowledge - autonomy, practitioner-orientation, informality, crossing boundaries_-are also characteristics that make them a challenge for traditional hierarchical organizations" (retrieved from www.ewenger.com, October 19, 2008).

The tension between tradition and innovation must be managed by appealing to teachers' innate impulse to improve practice on behalf of their students. As Barrett (2006) puts it, "[e]xperimentation with new strategies and the refinement of familiar techniques appeals to teachers' desires for change" (p. 22).

Groups that sustained long-term, multiple cycles of action-asking questions, gathering data or information to answer the questions, and returning to evaluate evidence and reflect — seemed to better facilitate transformative learning through changes in teachers' habits of mind and points of view. Establishing what to bring forward for group discussion, and when, and how, was problematic in several groups. What, in the way of conversation, materials, and artifacts was brought "within the [group] horizon of observation" (Little, 2003, p. 938) was notable both in terms of what was revealed and what was never shared. An area for thoughtful consideration is the need for accurate, transparent views into real classrooms - "publicly available features of practice" (Little, 
2003, p.918), and the need to accomplish this without threatening teachers' autonomy, privacy, or self-confidence. Junda's (1994) participants credited the videos of teaching with developing their skills of observation and instruction; the visits to their classroom by the instructor were cited as the most meaningful part of the project.

“Looking at student work” groups. In the last decade, professional development groups and school reform initiators have utilized structures for bringing teacher together to look at student work. In a two-year case study of three prominent national organizations that use formal protocols for engaging small groups of teachers in sharing and talking about student work, Little, et al. (2003) discuss factors that seem to help these study groups be productive. Little and her partners looked at an elementary school affiliated with Harvard's Project Zero, a middle school affiliated with the Academy for Educational Development, and a high school working with the Coalition for Essential Schools. These are all national school reform organizations that provided structured discussion guides ("protocols") to facilitate teacher learning groups; the mechanisms to direct the conversation differed somewhat between groups but all were designed to focus systematic attention on student work and organize the meetings toward productive, collaborative teacher inquiry.

An important feature of teacher study groups that formally examine student work according to Little et al. is that they "stimulate an open-minded but focused examination of what that work can tell teachers about student understanding and teaching practice" ( $\mathrm{p}$. 188). This indicates that teachers are helped to have targeted conversations around real, visible teaching practice and student outcomes; a missing element from several studies reviewed previously. 
In the groups studied by Little and her partners, facilitators used protocols designed to balance the work between easy, superficial talk and challenging questions. The facilitators consistently worked to deepen the conversations and take the talk about student achievement and teaching practice to the next level of introspection. However, the groups that were most successful in terms of "sustained and lively conversation" (p. 189) about student work were flexible enough to use the study meetings and protocols for local purposes. The teachers in the study group, not the facilitator, instigated the flexibility. This confirms the need for teachers to take leadership in their own study group and participate along multiple roles.

Little also found several familiar dilemmas in groups coming together to look at student work; problems which resulted in less successful, more "brief and tentative" (p.191) forays into teacher conversation. One was the need teachers had to tread lightly and avoid hurting feelings; teachers are conscious of the idea that student work is also highly reflective of teachers' work, and wanted to avoid hurting others' feelings. On the other hand, teachers often responded to each other's students' work by justifying or defending their own teaching practice. This suggests again that teachers need mechanisms for forthright talk and the avoidance of labels and methodological defensiveness. Another difficulty Little et al. observed was the lack of time for discussion of other issues; they found that teachers consistently wanted to start talking about wider topics in teaching and schooling. Little et al. asked "whether the impulse to talk about teaching might have been better served by sustaining closer and longer attention to the available evidence of student learning" (p. 191, emphasis in original). Finally, Little et al. found that many of the protocols in use in teacher study groups required teachers to 
choose and bring student work for sharing, and that teachers were often unsure of what to present.

Little et al. found the practice promising, mostly because of the way the meetings bring students into teacher talk more regularly and in a more formal manner. Between meetings, teachers reported looking at their world "with a different eye" (p. 187); reflecting on past conversations and thinking about presenting student work in upcoming sessions.

Looking at student work has the potential to expand teachers' opportunity to learn, to cultivate a professional community that is both willing and able to inquire into practice, and to focus school-based teacher conversations directly on the improvement of teaching and learning. (p. 192)

Several of the strategies above are intended to help groups of teacher-learners recognize conflict as inevitable, healthy, and a way to move forward. The more objective data presentation modes available within structured protocols may help learning communities to feel less threatening or opinionated. Group longevity plays a part in this as well: the long-term professional development groups described in this paper seemed to eventually work through dissonances to come to convergence on certain matters, and to find ways of working together that enabled the hard conversations without breakdowns.

A problem with some professional development structures is teachers get new ideas but are unable to implement them in context. Robbins' (1995) teachers initially struggled with making the transition from summer seminars to school-year demands, but as they were empowered through doing teacher-research, in the field, and by a strong inquiry-based collaborative community, they worked through these difficulties in the two-year study.

Just as students learn — through the conversations of critical inquiry as members 
of a community — so should our profession. "What we want for our children, we should also want for their teachers" (Hargreaves, 1995, p. 27). Teachers should have the opportunity to learn from others and be supported as members of a community.

\section{Collaboration in General Education}

Within music education and educational research in general in the last halfcentury, collaboration has been examined to better understand the processes of group interaction and how it may relate to student learning. This is a discussion of selected research on collaboration within educational settings, which provided useful background information for me as I organized the CTSG to study collaboration in the classroom.

In general, school-aged children are assumed to have both the social and cognitive skills to engage in collaboration and are theoretically able to be productive in many different collaborative group environments (Azmitia \& Perlmutter, 1989). PerretClermont, Perret, and Bell (1991) reviewed a large body of research credited with labeling interactions that contribute to or detract from efficiency in group problemsolving. This literature shows that interactive problem-solving generally surpasses individual efforts. Azmitia and Perlmutter, in their 1989 review of the literature, did not find a conclusive link between a child's level of skill and productivity in collaborative efforts. They stated that variables such as children's expertise, developmental level, style of interaction, social skills, type of interactive tasks, and instruction in collaboration need to be considered in this type of research.

The following five studies are subsequent efforts to define collaboration and codify what variables may affect the success of collaborating groups. The analysis of 
specific collaborative aspects in these studies was helpful in considering different ways to examine collaboration in music classrooms.

Roschelle (1992) examined a case of two high school students engaged in learning physics with a computer simulation. The two students worked together after school on the computer-simulated physics problem for two, one-hour sessions. Roschelle analyzed the conversation and computer-recorded actions of the students in terms of five episodes, or "durations of coherent activity" (p. 243). He found that the two students were able to cooperatively construct an understanding of the concept of acceleration. This shared understanding is an example of "convergent conceptual change"; they incrementally, interactively, and socially reached an understanding that was a large conceptual change from their starting point. The new understanding closely matched the correct, scientific meaning, and was shared by each student.

Roschelle credited several elements of the collaborative interaction with helping the students reach convergence. In this study, students constructed an abstract situation to help them understand the concept, using metaphors. They engaged in a cycle of iterative, conversational turn-taking structures that helped them "build on each other's ideas and intentions, draw new ideas into a common conceptual frame, and repair divergences" (p. 242). This allowed their meanings to be "negotiated, confirmed, and repaired at a suitably high standard of evidence" (p. 243). In other words, they continually confirmed their mutual acceptance of shared knowledge by continuing to further elaborate on the other's stated knowledge, not simply reciting it back, affirming it, or just going on to the next topic. After working to solve the physics problem, they discussed it with an interviewer 
in terms of shared conceptual change: they described "what 'we thought', why it was wrong, and what their new conception was" (p. 262).

Roschelle's work provides a theoretical framework with which to analyze patterns of collaborative interactions: one which differs from Vygotskyian (1978) and Piagetian (1950) theory. A full accounting of the principles espoused by Vygotsky and Piaget is outside the scope of this review. Briefly, Vygotskyian theories of collaboration center around the way an experienced peer scaffolds knowledge acquisition for her less-expert partner, so that the inexperienced novice can appropriate the expert's knowledge. This model focuses on the way knowledge can be transmitted from person to person.

Piagetian theory (1950) holds that knowledge development occurs slowly and through a disruption of equilibrium. Cognitive conflict in a social setting, as people work together, fosters a confrontation of divergent points of view, and it is this conflict that results in individuals restructuring their conceptual understanding.

Both of these theories lack a full depiction of how meanings can be socially constructed, and how meanings are shared throughout the process of creation. Roschelle (1992) asserts that in the case of the two students, a "convergence account" (p. 272) provides a more complete picture of conceptual change through collaboration. Roschelle constructed a four-part theoretical framework to explain the process of convergence: (a) people construct a situation, abstracted from the literal features of the world, which they can consistently refer to in terms that are unique to the group; (b) people use metaphorsin terms of the constructed situation, and relation to each other-to explain things; (c) people try to converse in ways that make knowledge visible, monitor the other's knowledge, and repair any discrepancies; and (d) people working together collaboratively 
seek higher standards of evidence of convergence, as they continue to work. In this study, for example, the students' standard of convergence was so high, they grew to be able to complete one another's sentences and elaborate on the other's emerging thoughts.

Roschelle and Teasley (1995) used the idea of a "Joint Problem Space (JPS)" (p. 70) to analyze one dyad's collaboration. The dyad was two 15-year-old males working together on a physics problem, using a computer simulator. The researchers asserted that collaborative problem solving would occur in a shared conceptual space, one negotiated and constructed by the two participants through their language, actions, and environment.

To demonstrate the nature of this collaborative problem solving within the JPS, the researchers carried out a detailed analysis of the students' interaction as they worked together to solve physics "challenges," (p. 72), or problems. When the students finished the challenges, they were interviewed about their learning.

The boys worked on the simulator, called the "Envisioning Machine" (p. 71) for three, 45-minute sessions. This analysis concerns one particular challenge, which the students worked on for eight minutes. In this challenge, the students had to adjust the direction and speed of velocity and acceleration vectors on a particle, and then run the computer simulation and checking the particle motion. The goal was to match another motion displayed on the simulator.

Roschelle and Teasley used a specific definition of collaboration in order to clearly describe the phenomena under study: "Collaboration is a coordinated, synchronous activity that is the result of a continued attempt to construct and maintain a shared conception of a problem" (p. 70). To analyze the nature and quality of the students' collaboration, Roschelle and Teasley constructed a framework to understand 
how the students jointly carried out two activities: building a JPS and solving the problem.

The JPS, according to Roschelle and Teasley, is a socially shared set of understandings which emerges when students engage in collaborative problem solving. It allows for productive conversation about the problem. The JPS is built through conversation: specifically, through introducing knowledge, accepting it into the JPS, checking on-going processes for any divergences in meaning, and repairing those meanings that hamper the problem-solving progress. The researchers identified five conversational structures that help construct and maintain a JPS, and analyzed their participants' interaction for their occurrence. The structures are (a) turn-taking; (b) socially distributed productions, which is a sentence started by one partner and finished by another; (c) repairs, or attempts to resolve "breakdowns in mutual intelligibility" (p. 78); (d) narrations, or informing one's partner of intentions behind actions; and (e) coordination of language and action, including gestures.

In this study, Roschelle and Teasley found examples of all five structures in the students' interactions, and the researchers and the students agreed the problem was solved successfully. In fact, the researchers characterize the pair as "one of the most collaborative dyads we have studied" (p. 71). In their transcript and analysis of the pair's activity, a clear picture of dynamic, lively interaction emerges.

However, the collaboration was not altogether smooth. There were rocky divergences, lack of attention by one partner, unequal introduction of strategies and ideas, and rough turn-taking. At times one student seemed to be talking out loud to himself, not to his partner. At one point a breakdown occurred and one of the students began to play 
with the equipment. Through action, narration, and questioning by the other partner, the distracted student returned his focus to the project.

The overall construction of the JPS succeeded despite these difficulties. The challenge started with individual contributions of different ideas, and ended with a shared conceptual understanding of velocity and acceleration. The students were able to explain this new knowledge to the researchers in an interview.

Roschelle and Teasley point out the "fragility of the collaborative learning process": "[c]ollaboration does not just happen because individuals are co-present; individuals must make a conscious, continued effort to coordinate their language and activity with respect to shared knowledge" (p. 94).

Fischer and Mandl (2005) built upon Roschelle (1992) and Roschelle and Teasley (1995) in their study of computer-supported collaborative learning in pairs of students. They set out to discover the extent of the tendency for collaborative learners to converge, and what effect a "shared representation tool," (p. 410) would have on convergence. Another variable in the experiment was the shared representation tool; it was either content-specific (a graphical mapping tool specifically designed to allow the creation of a visual representation of ideas, theories, or discussions) or content-independent (a simple graphic editor program). The researchers wanted also to compare face-to-face and videoconferencing collaboration.

Sixty-four university students volunteered to work on complex problems in the field of education. The problem featured a theoretical background text and a case study. Their task was, with a partner, to prepare an evaluation of the case from the theoretical perspective in 25 minutes, using the graphic tool to represent important elements of the 
case and theoretical concepts related to the problem. The pairs were assigned to one of the graphic representation tools, and assigned to communicate either face-to-face or through videoconferencing. After the task, students were tested on factual knowledge of the theory text, and tested on their ability to apply the theory in a similar case.

Convergence outcomes did not differ significantly in the videoconference versus face-to-face settings. Pairs using the content-specific graphic tool used and elaborated more concepts than did the pairs using the less complex, non-education-specific graphic editor. The researchers found that collaboration within the pairs was not a strong indicator of the amount of shared factual knowledge they had at the end of the task. However, shared application-oriented knowledge — defined as transferable knowledge, indicating higher order thought processes — was fostered by collaboration. They also found that while the collaborators tended to converge in both processes and outcomes, there was a stronger tendency towards convergence in aspects of the process of learning (i.e. their use of resources, or their task strategy) not the outcomes (shared understanding in the post-test). They concluded that support, in terms of a collaboration script, prompts, or scaffolds, would facilitate a more effective task-completion strategy by helping pairs learn to create a coordinated effort to come to consensus.

The idea of learning to create consensus was mentioned by the researchers in each of the four studies above. Negotiating, turn-taking, and repair of divergence contribute to productive collaboration. The construction of a successful shared problem-solving space requires effort from each of the collaborators. But as teachers know, groups of children are not always willing or able to make this coordinated effort. 
The next study in this review (Barron, 2000) is an examination of two collaborative groups, one of which was not successful. The researcher concludes by calling for support for teachers, like those in the CTSG, who seek strategies for enhancing the ability of their students to collaborate.

Barron (2000) studied the difference in problem-solving outcomes between two groups of three sixth grade boys. Barron alludes to prior research on group interaction that has focused on individual contributions and individual learning outcomes, excluding valuable information on the group as unit of analysis. She contends that researchers need to continue to create vivid pictures of interaction, as in Roschelle and Teasley (1995). These depictions will help to capture, describe, and explain the relationship between the type or nature of collaboration in a group and the intellectual gains or work that happens in that group.

By focusing on the group or 'ensemble,' it is possible to provide descriptions of interactions that capture the dynamic interplay in meaning-making over time in discourse between participants, what they understand, the material resources they have available and choose to utilize, the type of contributions that they make and how those are taken up in a given discourse. (Barron, 2000, p. 406)

Barron examined 16 groups of sixth graders working together for four hours on a problem-solving task. The task involved complex trip planning and math problems: students were instructed to figure out how to get the main character in a video series, The Adventures of Jasper Woodbury (Cognition \& Technology Group at Vanderbilt, 1997), home safely in a motorboat, with enough gasoline. The triads each had a workbook to organize their progress, and a set of 18 stills from the video in a storyboard. Students participating in the study were all male, and had high math scores on a standardized achievement test. They all studied math with the same teacher. 
Barron chose two groups to examine closely: one that received full credit on the task, and one that received only one-fourth the possible points. She analyzed the structure of the triads' interactions in order to understand why successful collaboration might or might not occur. The unsuccessful group was competitive, individualistic, used selffocused talk, struggled for control, wrote little in the workbook, and brought different expectations to the project. They did not use turn-taking, negotiation, and faltered in creating any sort of shared understanding of the problem. This interrupted Roschelle's (1992) cycle of sharing, repairing, and mutual making sense. The successful group was respectful, made eye contact, used the workbook as a center of coordination, and coconstructed solutions-the participants made incomplete statements or assertions and others continued to fill in the rest or complete the idea.

In conclusion, she identified three forms of coordination that seemed to contribute to successful collaboration. Markers of their presence are seen along a continuum of high to low (Barron, 2000, p. 429):

1. Shared task alignment: group is collectively oriented toward problem solving. Solutions are co-constructed and members reference one another's ideas.

2. Joint attention: Attention is jointly focused during solution-critical moments.

3. Mutuality: All members may potentially contribute reciprocally and meaningfully. Conflicts are productive, members engage with each other's ideas, and dialogues are transactive. 
Barron suggests that teachers help students develop strategies for (a) noticing their own and others' attentional engagement; (b) maintaining common focus; (c) persist and remain engaged in the midst of confusion and lack of understanding. She recommends explicit reflection on the connection between communication processes and final products. She concludes:

We still face the challenging problem of establishing that participation in a community of practice is a genuine and important form of learning. It would be powerful to demonstrate that discourse-rich classrooms that routinely engage students in joint work allow different and better ways of collaborating (p. 433).

Barron recommends further study on helping teachers learn more about student collaboration: "Helping teachers create opportunities for students to develop their capacities for productive engagement in collaborative problem solving is both an educationally and socially important venture" (p. 433). The CTSG was such an effort to help teachers.

Palincsar and Herrenkohl (2002) analyzed more than ten years' worth of research on settings that attempted to encourage peer learning in collaboration. They focused on two bodies of literature, that on reciprocal teaching (RT) for reading comprehension, and an inquiry-based science education method called Cognitive Tools and Intellectual Roles (CTIR). Much of the research has been done at the classroom level, and the authors point out that in addition to domain- and knowledge-specific concerns about collaboration, the social culture and collective history of a classroom must be considered. The individual histories of students and their experiences working together may have a strong impact on collaboration.

In synthesizing the research on these two learning interventions, Palincsar and Herrenkohl conclude that the effort to transform classrooms into collaborative learning 
environments is dependent on many complex social issues that need more research in actual classrooms: "[U]ntil we understand more fully how to socialize students into new ways of dealing with peers as intellectual partners, it is unlikely that this research will make much of a difference in the real-world experiences of teachers and students" ( $p$. 32).

Kynigos and Theodossopoulou (2001) found similar themes in their attempt to identify real-world obstacles to collaboration within groups of elementary school learners. This study was a look at four groups of same-gender students, two in third and two in fifth grade, working on a graphic design and programming project. The researchers set out to find how members of the group perceived themselves and their peers during the collaborative activity, what role the computer-based environment played, and to discover the social norms regarding collaboration in this class.

The negotiation of social roles was problematic and confusing to the children; the researchers describe role negotiation as an "overbearing presence." Students saw the teacher as a referee or director. Students considered their collaboration to be positive only if it had a lack of visible conflict. Admitting to mistakes or being critical were seen as negative according to the social norms entrenched in the group, even though they may have aided in coming to convergence. The groups resisted the establishment of collaboration as central to classroom learning. The authors found that "even though pupils do engage in constructive and social activity, they still seem to feel that these are not recognized as valid and so they try to conceal that they happen" (p. 72).

The use of the computer was ineffectual; students felt time spent exploring and learning the program slowed progress toward the final goal. The computer aided uneven 
role distribution and power in the group as quicker, more advanced users took charge. The researchers state that their results should not be seen as "lessening the possibilities and the richness of such an environment for both cognitive and social development" ( $p$. 72). Instead these findings shed light on classroom collaboration and the need to develop practical strategies for incorporating it.

Chinn, O'Donnell, and Jinks (2000) conducted a quantitative study of the type of discourse structures that emerged when 109 fifth graders, in groups of four, wrote and discussed scientific conclusions. Students were first asked to write their own individual conclusions about electrical circuits they constructed. Then they were provided with three other conclusions - written by the researchers to be intentionally good or poor - to evaluate in the group. Half the groups were asked to discuss which conclusion was best/worst; the other half were asked to discuss which conclusions were OK/not OK. The researchers theorized that the best/worst task would promote more discussion, because it requires explicit comparisons to be made. Finally, the students completed a transfer task: writing conclusions about experiments they did not do themselves but that were displayed in pictures.

The researchers analyzed transcripts of the discussions to determine if students collectively construct an "argument network" and if so, how it was structured. A discourse analysis based on the argument structure of the group revealed that complex argumentation promotes learning, as measured by student performance on the evaluation of the transfer of conclusion-writing skills. Complex arguments constructed by students on their own, and those constructed with the help of others, with or without counterarguments, all significantly positively affected post-test results. The best/worst 
task yielded significantly more complex argumentation. The OK/not OK task encouraged a process in which students were content with finding a quick, acceptable answer.

Finally, the number arguments that featured simple reasoning negatively correlated with post-discussion performance on the transfer task. Elaborating on knowledge by including other evidence and explanations was associated with learning.

The elaboration of conceptual knowledge, another aspect of collaboration that seems to result in group construction of a shared understanding, was also identified by van Boxtel, van der Linden and Kanselaar (2000) as positively influencing learning in their study of collaborating physics students.

van Boxtel et al. studied 20 pairs of high school physics students in two different schools. The researchers wanted to investigate how collaborative tasks would elicit verbal elaborations, and in what frequency. They wanted to find out if the amount of elaboration during collaboration was related to individual learning outcomes, as well.

The dyads were same-gender, and randomly assigned. Each student was given a pretest testing their knowledge in using and communicating the meaning of scientific concepts about electrical circuits. One week after the pretest, randomly assigned dyads were asked to make either a concept map or a poster about the same scientific concepts. The concept map, a more abstract project, was a network in which concepts, symbols, formulas, and graphs are joined with lines that explain the links between them. The poster was a drawing (of an electric torch) in which each part had to be explained using the concepts. The researchers thought the poster project would elicit more talk about relationships of concepts in a more concrete way. 
Another variable in the study was individual preparation. Twenty students, comprising ten dyads, were asked to prepare individual designs for their concept map or their poster in advance. They were given only five minutes of time for this individual preparation. Each dyad, whether they were assigned to the individual preparation condition or not, was given 45 minutes to complete the task. The interactions were videotaped and coded for episodes of utterances of propositions (making a statement about the meaning of a concept), questions (followed by no answer, short answer, or elaborated answer), and conflicts (non-confirmations, counter-arguments, and critical questions). The students were then given a post-test, very similar to the pre-test, the next day.

Among the findings was that individual preparation time had no effect on the amount and types of conflict utterances, nor on the post-test scores. The preparation did increase the amount of questions in both groups. The designs prepared individually also gave the students another tool with which to explain and support their own ideas for the dyad's collaboration. Dyads who worked on the concept map discussed the relations of concepts more intensely; dyads working on the poster spent longer periods of time writing and drawing.

In the post-test, students in both groups (concept map and poster) gave better and more complete explanations of the electrical concepts. There was a positive correlation between the amount of elaborative discussion in the dyads and scores on the post-test. Even though the task lasted only 45 minutes, and students had only one another for resources, answers on the post-test were more thorough; students were better able to recognize, explain, and apply concepts. 
The researchers concluded that elaborative and collaborative interactions are helpful for learning concepts. Elaborative discussion, when students not only reflected upon and elaborated their own understanding but also integrated or elaborated the input of their partners, helped the students focus on understanding the concepts in question and co-constructing a shared understanding.

This study makes a helpful distinction between the elaboration of conceptual knowledge and possible convergence that may occur with collaboration, rather than the effects of collaboration, as might be measured in cognitive, social, or affective outcomes. These researchers distinguished between collaborative learning as a method, marked by an attempt to facilitate learning with a collaborative task (and measured by individual scores afterward), and the process of collaboration. The researchers found that the process of collaboration is highly impacted by the open-endedness of questions asked, and that while some tasks stimulate most students to talk about and elaborate on the concepts, there is no guarantee that all students given the opportunity to collaborate will participate in "high-quality discourse" (p. 328) leading towards conceptual convergence. However, they also stated that "it is not the shared understanding that is the most important for learning, but the effort to realize it" (p. 328). As a result of this study, the researchers recommend further study into the nature and quality of student interaction in dyads, as the elaborative, collaborative interactions seemed particularly promising for actively stimulating learning.

Collaboration does not necessarily entail working in small groups. Entire classes can be guided in collaborative endeavors, and from a social constructivist perspective, it 
is the quality and substance of the nature of these interactions that may stimulate students' higher-order thinking (Palincsar, 1998).

Forman, et al. (1995) discuss the role of the teacher in creating a whole-class collaborative discussion environment, one which goes beyond initiation-responseevaluation patterns. Forman et al. analyzed two hours of interaction in a middle school mathematics class. Small group work accounted for $55 \%$ of the time, $15 \%$ of the time was spent in student presentations, and $29 \%$ of the time was devoted to what appeared to be teacher-centered discussion. However upon further analysis of the teacher-led direction, Forman et al. determined that the teacher's interactions with students were facilitative, not directive.

The interplay between a classroom environment, task, and student capability is complex and subsequent researchers have attempted to define ways in which to foster student engagement and learning within participatory, collaborative classroom environments. Kovalainen and Kumpulainen (2007) studied a 17-student elementary school classroom in Finland, aged 9 to 10 years. The researchers videotaped nine hours of classroom interaction, three hours each from math, science, and philosophy lessons. They completed a micro-level analysis on each episode that featured whole-class interaction. In total, the researchers identified 1,675 "message unit," or the smallest unit of conversation. In a larger-level analysis, the researchers identified "interaction moves," or bigger chunks comprised of thematically linked message units. These interaction moves were labeled teacher- or student-initiated, bilateral (between two students or student and teacher) or multilateral (between several students and the teacher). 
The researchers' analysis indicated the presence of four distinct modes of participation. Vocal participants (four of the 17 students) were authoritative and took initiative in starting and responding to discussions. They enthusiastically created the framework and direction for classroom interactions. Responsive participants (five of the 17) participated in dialogue, and provided substance for classroom discussions by responding to other's arguments and thoughts. Bilateral participants (five of the 17) responded to the teacher or one other student. Their contribution to whole-class dialogue was significant in providing elements of evaluation and reflection to the discussion. They rarely joined in multilateral engagements, even though their participation as initiators of new topics was significant. Silent participants (3 of the 17) contributed only when prompted by the teacher; however, their short and often informative responses helped to either clarify discussion or open up new topics for the other three types. After initiating the dialogue with the silent participants, the teacher would rarely engage in bilateral discussion; instead, she would quickly open the discussion to the entire class.

Kovalainen and Kumpulainen found that these roles generally stayed consistent for each student, through each subject. They assert that in order for classroom communities to be vibrant, truly collaborative entities, classroom communities should provide and encourage participation across various modes. For example, bilateral participants could be supported to participate more spontaneously and generally, perhaps by working in a smaller group first. Responsive students could be helped to participate in more analytical ways, and coached in how to initiate dialogue. Silent students' social skills were seemingly related to their lack of frequent participation. Silent students' participation in collaborative discussion could be enhanced gradually by a thoughtful 
teacher or peer coach. Vocal students could be made aware of the diverse modes of participation and how each contribute to the good of the classroom community.

The teacher would also interact differently with students of various modes. She would remove herself from discussions that were student-led (usually by the vocal participants). She provided intense scaffolding for bilateral and silent students to enter the discussion, but did not provide help for them to maintain a collaborative presence in the dialogue. She also regulated turns in speaking more when bilateral participants were entering the discussion.

The researchers summarized their findings by stating that engaging students in meaningful collaborative communities "requires more than creating a friendly, safe environment for learning" (p. 156). The teacher's role in working with students of various participatory modes is to provide "analytical and social support...taking into account [students'] personal characteristics" (p. 156). The researchers explained that these scaffolding efforts are positive but that:

[t]eachers should be all along aware of the changing nature of the instructional context by carefully monitoring the social interactions of the learning community, recognizing students' diverse needs on the moment-by-moment basis and adjusting their scaffolding...in order to provide flexibility and room for possible role change. (p. 156)

Summary. Several researchers in this section discussed the need for further classroom applications of collaboration. The idea that social conflicts, and resistance to working together, may hamper student collaboration — as in Kynigos and Theodossopoulou (2001) and Palincsar and Herrenkohl (2002) — is important information to know about structuring collaborative activities. 
The characteristics and definitions of collaboration —including concepts such as convergent conceptual change, joint problem space construction, shared task alignment, mutuality, and engagement-may translate into the music classroom from the disciplines that dominate this body of literature (math, science, and reading). The characteristics and definitions of collaboration that emerged in the CTSG discussions are compared with this literature in Chapter Six.

The above category of research also helps define types of collaboration: dyad or triad activity, to small group problem-solving, projects and tasks, to whole-class activities. Music teachers often have the challenge of instructing large populations, which requires a different mindset regarding how to determine and scaffold individual students' participation mode as in Kovalainen \& Kupalainen (2007). The next section of the paper describes research on the applications of collaboration in the music education environment.

\section{Collaboration in Music Education}

Research on collaboration in the music education setting has focused on children's collaboration during the compositional process, and on the way students construct shared meaning of a problem solving task. Each of the six studies in this section observe in some way the sociocultural phenomenon of students constructing a shared meaning of a piece of music as they compose, arrange, or improvise.

Few music education researchers have studied the nature and type of collaboration that occurs in other types of student music-making in an effort to understand the value of structuring collaborative experiences for students, or to examine the sort of learning outcomes that might accrue as a result. St. John's (2006) is the only 
study in this review that looks at collaboration within the context of an entire music classroom, and which does not involve a specific composition or improvisation project. Other studies in this section involve student chamber music environments.

DeLorenzo (1989) studied the creative problem-solving processes of students in four different sixth-grade general music classes. The classes, which she videotaped one to three times in a single school year, contained 25, 12, 28, and 17 students, respectively. The researcher did not ask the music teachers at each school to arrange a specific activity for videotaping, rather, she asked the teachers to contact her when the class would be doing a "creative project" (p. 192). She ended up with data from eight projects, including videotapes, written accounts of each class, teacher-made handouts or assignments, and transcriptions of musical responses.

DeLorenzo's primary area of investigation was the problem-solving of individual students. Although seven of the creative projects in her data set involved small group interaction, DeLorenzo's main unit of analysis was the individual, not the group. She studied the exploratory improvisations, elaborations, and manipulation of motives by individual students within the groups.

However, in corollary findings, DeLorenzo found that students involved in a group problem-solving process used analysis, synthesis, evaluation and inquiry. Of particular importance in DeLorenzo's findings was that students made a series of musical choices to solve interesting, motivating, and level-appropriate musical problems. Successful students placed a premium on musical expressivity in their solutions. However, students who were not successful and lacked evidence of meaningful involvement in the process seemed to DeLorenzo to need additional, structured practice 
in the domain of musical thinking and problem solving. This seems to echo van Boxtel, van der Linden, and Kanselaar's (2000) finding cited above: that while the learning environment and student capacity affect the collaborative process, the quality and type problem to be solved is of great importance as well.

For her study of shared musical understanding, Wiggins (2000) returned to data gathered for previous studies between 1990 to 1998, as well as 600 audio- and videotapes from her years as a general music teacher. For this particular investigation, she selected data from six instances of elementary school group composition and improvisation. She examined it for evidence of "shared understanding," defined in this context as "a group's vision of a work in progress" (p. 85). She also sought negative evidence. Negative evidence occurred in two ways: instances where a group's composition or improvisation appeared to be successful but there was no indication of shared understanding, and instances of unsuccessful products, but high indicators of shared understanding. Wiggins concluded that in "particularly successful student work, the elements and nature of shared understanding were particularly clear" (p. 66).

Of Wiggins' six data sets in this study, four involve group work: (a) whole-class (25 students) song composition project, (b) a duo blues improvisation, (c) a group of three students composing a recorder piece in ABA form, and (d) a group of five students composing an original song. The remaining two are a student-teacher activity, and a student composing alone at the keyboard.

Wiggins found that students engaged in successful collaborative interaction, within large or small groups, seemed able to come to shared understanding as reflected in their processes and products. Wiggins found several commonalities among the groups. 
Students shared, extended, varied, and answered one another's ideas verbally, nonverbally, and musically. They drew upon the community musical knowledge for ideas, and kept track of ideas by singing and playing. Like students in Roschelle and Teasley's (1995) study, they expressed ideas in joint musical statements: begun by one student and finished by another. Several groups seemed to establish a safe, friendly atmosphere for creating by engaging in what seemed like off-task talking. Students received immediate feedback from others, causing them to "restate, justify, defend, or alter their ideas...generat[ing] within the individual a sense of possibilities" (p. 86).

Wiggins asserts that collaboration can broaden an individual's musical knowledge base and strengthen her ability to initiate musical ideas. In her conclusion, Wiggins makes a strong case for teachers to plan instruction that will "create opportunities for students to share musical ideas...to both accept and encourage the verbal and musical conversations that occur among students as a productive part of their music learning experience" (p. 87). Wiggins' recommendation for teachers is very similar to Barron's (2000) call to help teachers create space for collaborative work.

Burnard (2002) observed 18 twelve-year-olds in a weekly improvisation club. The club met 21 times during the lunch hour of a middle school in London. Burnard's intent was to study the musical and verbal communication of the students, and to determine what meaning the children ascribed to their communication. To that end, her data collection incorporated focus group interviews to encourage student reflection, as well as videotapes of the club meetings. She also notated improvisations in order to compare what was said or reflected upon, with what was done musically. 
Burnard's analysis centers around six chronological improvisations: one solo, four whole-group, and two trios. After the first student broke the ice, the rest of the students joined in. They, variously, made recommendations to either coordinate their entrances and regulate the improvisation, or to "just get in when we are ready to play something" (p. 162). The third improvisation featured students negotiating leadership and supporting roles; the resulting music was oriented around a leader's loud, simple drum beat that made it easy for others to join in.

Burnard noticed a "new experiment in musical interaction" (p. 163) in the fourth improvisation: "a series of free and spontaneous exchanges" (p. 164) between players. The first three improvisations incorporated discussion about how to start, continue, and stop, according to a leader. In the fourth, the process changed. The leader continued to try to assert himself by getting the group to stay with his regular beat and stop on command, but the rest of the group wanted to carry on and dissolved his leadership by freely interacting and exploring ametrical rhythms. The dissolution of leadership was followed by explicit negotiation of the students would take turns starting and stopping, and how ideas could be better heard and incorporated that way.

In improvisation five and six, "a flurry of players seeking, attaching and settling into new groups" (p. 164) resulted in two trios being formed. Interestingly the new groups followed the negotiated conventions agreed upon in improvisation four.

In subsequent reflection, Burnard found the students described their improvisation as incorporating shifts in focus, as they attended to one another's ideas. They reported conveying musical information through "communicative intention" (p. 166), meaning 
that they affected one another by making adjustments to timing and blend so that their parts would coordinate.

Burnard found that the students' verbal discussion during and after the improvisation was as interactive as the music-making, and characterized the interactions as spontaneous and fluid. She relates another powerful example of sociocultural influence when she says that the meaning of each improvisation was not "generated within one mind, but rather, constructed between people" (p. 168).

Burnard relays three points important for practical application of this research. First, the instrument choice was important for the students, as they selected comfortable and physically easy media — in most cases, percussion and recorder — with which to express themselves readily and fluidly. She states that teacher intervention is necessary at the point where the group or any member of the group is unable to contribute with continuity and ease.

Second, Burnard emphasizes the importance of trust and empathy in the classroom. For Burnard, the teacher can achieve a safe environment of spontaneity by stepping back, intervening through questions not commands, and lead in a way that empowers student responsibility.

Burnard also recommends that the students have regular, specific venues with which to discuss the improvisation and the interaction. In this study, the reflective focus groups gave the students needed opportunities to talk about and process their own and others' views. Effective teacher questioning can empower students with freedom in this arena as well. 
For Burnard, the group improvisation gave the children a unique way to give meaning to their creativity; the intensity and immediacy of their expressivity was enhanced and made tangible by the social interaction with the group. She argues for an inclusion of group improvisation and musical interaction within the curriculum because children experience "thought as a function of action" (p. 169) and only by allowing them to make music together will children be fully able to explore the richness of musical experience.

Dillon (2003) examined the type of dialogue when students collaborated in groups of two and three on open-ended music composition activities. She observed 42 high school students in a secondary school formal music class and at a community center during after-school club meetings. 30 pairs and 12 triads were asked to compose a piece of music using a sampling and sequencing software program.

Videotapes were made of all interactions, transcribed, and coded according to their functions. The four most common utterances, in both the formal and informal settings, were musical suggestions, musical extensions, positive support, and questions. Dillon concludes that the absence of explanatory dialogue and conflict is evidence of shared understanding.

Dillon found that collaboration in a creative task is characterized by these elements: students making suggestions, extending previous ideas, voicing support for and agreement with ideas, and questioning. The discovery and exploratory aspect of a student composition activity created an atmosphere in which students quickly established shared understanding of their goal and engaged in a cyclical process of trying ideas and evaluating them. In this task, co-construction of meaning is described by Dillon as absent 
of "detailed engagement in extended argument, counter argument, justification sequences, where opinions and solutions have to be made explicit as the partners try to solve a problem that is already defined" (p. 895).

Dillon's conclusions are different from the findings pointing to the need for divergence and discrepancy repairing (Roschelle, 1992; Roschelle \& Teasley, 1995) and positive arguing (Chinn, O’Donnell, \& Jinks, 2000). She attributed the lack of conflict to the fact that the ill-defined "problems" in music composition do not lend themselves to "the problem solving closure activities typical of well-defined tasks" (p. 895). In other words, collaborating students seem to quickly establish shared meaning and stay in an idea-generating/evaluation phase when the task has no right or wrong answer.

The lack of a definitive "correct answer" is one way in which the research on musical collaboration differs from research on students who are working on physics, mathematics, or reading tasks. The matter of "problem-solving" in music, when the "problems" are not readily identified nor neatly completed, makes the research in music composition and improvisation somewhat difficult to compare with more categorical endeavors.

The following two studies are a look at collaboration from the standpoint of student interaction to identify, solve, and evaluate musical problems. Both studies also contain discussion on the role of conflict and disruption in this arena. Younker and Burnard (2004, 2008) have studied collaborative composing and collaborative arranging with a focus on the interaction between students. In the 2004 paper, the researchers describe two cases: an elementary school classroom (students aged 9 and 10) in the United States, and a secondary school classroom (students aged 12) in the United 
Kingdom. The level of teacher involvement with the composition activities in the two settings varied. Some activities were teacher directed, but the students were able to establish their own parameters within the creative process. Some activities were completely student-led. Younker and Burnard collected verbal reports from students and teachers in each setting. They also, in multiple observations, recorded musical utterances, and student perceptions through think-aloud protocols and unstructured interview techniques.

An analysis of student talk reveals a sequence of problem identification, solution generation, and solution evaluation. Within this sequence, the forms of interaction were characterized in the rules, roles, and focus self-defined by students. These interactions were verbal and non-verbal.

For example, in terms of rules, verbal interactions governed assignments of parts. Those with more musical knowledge were more verbal. However, students playing instruments, or even disrupting the talk with instrumental sounds, also contributed to the negotiation of who would play what part. In terms of roles, those students who took leadership roles would often verbally dominate, and in some cases, argue over which musical parts should be included. They found evidence also that students would peermediate these conflicts. Non-verbal establishment of roles was characterized by dominating playing and conducting gestures. Focus was verbally evident through arguing, peer-teaching, and problem-solving. Focus was non-verbally evident through exploring musical parts, practicing, and musical modeling.

Another important element of these interactions was the nature of the teacher interventions. The teacher would guide students in a learning process, through observing 
and describing, asking students to describe and reflect, and probing for clarity or to resolve discrepancies. The researchers found that through this process, the teachers helped the students learn reflective thinking. The teachers would also teach non-verbally through modeling, playing, and singing.

In these collaborative processes, self- and group-identities as composers were formed and students gained insights about composing. The researchers found that future studies of collaboration are needed to better inform pedagogy in areas of facilitating musical growth, musical identity, and creativity. Specifically, the researchers suggest that the nature of feedback and reflection in student interaction be made explicit, and the roles of teacher as facilitator, observer, and teacher further identified and understood.

Younker and Burnard's (2004) work is important in the context of this study because it sets a precedent for examining student-student and teacher-student interactions, both verbal and non-verbal. It provides valuable information on the verbal and non-verbal ways students and teachers take on various guises within collaborative interaction, which was an important part of the CTSG's work as we examined classroom video.

Younker and Burnard's (2008) study applies Engestrom's (1996) Activity Theory (AT) as a lens for interpreting the systems of interaction in fifth and eighth graders who were collaboratively composing and arranging. Using data collected for previous studies, in this work the researchers analyzed two different classroom musical activities, one in the United States and one in the United Kingdom. The United States group was comprised of eight participants, aged 10 and 11, composing collaboratively in samegender pairs. The United Kingdom participants were aged 12 and 13, arranging 
collaboratively in four groups of four students. Only one of the four groups was mixedgender.

Engestrom's (1996) theory is used in group-level units of analysis. The theory provides a way to understand how an entire group works on an activity, while socially and culturally mediating the processes as an entire "activity system." An activity is seen as a task in which subjects work on objects in order to obtain a desired outcome. In order to do this, the subjects employ tools. In addition to subject, object, and tool, three other interdependent elements make up the activity system: community, division of labor, and rules, or conventions.

The researchers were the first to apply AT as an analytical framework to music educational settings. They found that AT was a valuable theory for understanding differences in peer collaboration activity systems in different contexts. AT allowed the researchers to examine the dynamics of interaction between subjects (the students), tools (instruments), and object (task of composing or arranging). They also were able to look at the entire system's community, division of labor, and rules.

Students' prior knowledge figured strongly in aspects of subject-related collaboration. In the composition task, pupils with more musical knowledge were more verbal, and their prior knowledge was linked to their choice of instruments. In the arranging task, group work varied depending on the musical training of its members and the group's prior social knowledge of each other, as peers.

In tool-related aspects of composing, the researchers found the group played and modeled on their own and others' instruments. They used gestures as tools to cue tempo, beginnings and endings, and entrances. In arranging, the researcher found tool use 
determined how they mediated the task, and the ways instruments were used as tools depended on how much training the members had.

The researchers found object-related aspects of collaborative composition to be singing, playing, conducting, and moving; also, the students would act on the object (the task of composing) verbally by talking about music theory. In arranging, the object was acted upon by episodes of playing, practicing, drilling, rehearsing. The object was strongly acted on by the technical ability of the subjects.

Community aspects of composition involved the group making understandings about music, participation, and cooperation explicit. The community aspects of arranging were more focused around the way the group dealt with values, social, and working relationships. In the arranging task relationships faltered due to disagreements and lack of support for learners from their peers in the activity system.

The researchers defined several ways labor was defined in the composition activity system. Choice of instrument determined role. The labor was divided between evaluator (observer), mediator (teacher or pupils defusing tension and refocusing attention on the task), and teacher (peer teaching, modeling). The labor of describing, clarifying, disagreeing, agreeing, negotiation, problem-solving, and evaluation was also divided and taken on by various subjects. Within the arranging system, the division of labor acted to created a playable result (each person taking on a certain part). This resulted in cooperation or conflict.

In the composition task, rules (conventions) centered around the relationship between student roles. Rules were adapted for each participant. In the arranging task, rules were communicated through action and talk and reinforced through transactive talk 
between friends. A convention or rule for the groups made up of friendly peers involved all of them using their instruments as tools for thinking, not just one dominant person.

In this study the activity was the unit of analysis, not the group. The researchers were primarily interested in comparing actions and discourses between two activity systems: collaborative composing, and collaborative arranging. They were also investigating how Engestrom's AT could be applied to the study of activity systems in the music classroom.

However, in their findings are several elements that were helpful in the findings for this study. Collaboration happened more frequently when negotiation was present and the group was task directed. Their findings concur with Dillon's (2003) regarding the value of mutual questioning, supporting, and elaborating on peer's ideas. Collaboration involved "cumulative rather than disputational types of talk, where participants engaged with each other's ideas, extended them, and established shared goals and meaning." (Younker \& Burnard, 2008, p. 13). In both systems, the most experience tool-user was allowed to dominate, implying that levels of musical knowledge should be considered when creating collaborative groups: "[w]orkloads can turn asynchronous when unevenly divided between players" (p. 15). Also, the researchers pointed out that levels of friendship and interdependence in the group highly influenced the interactions.

St. John's (2006) study is a look at the way preschool-aged children (ages four and five) might acquire, and benefit from, the skills of musical collaboration. It also is the only study in this review that looks at collaboration within the context of an entire music classroom, and which does not involve a specific composition or improvisation project. In this research, St. John defines collaboration as "collective musical engagement" and 
finds examples of it in groups of all sizes, engaging in many different types of musicmaking.

St. John states that social aspects of the classroom community play a crucial in how children learn music, and in fact, that the type of communication necessitated in a musical environment is an ideal microcosm for learning collaboration:

Awareness of others and how each relates to the others in the dynamic interplay that occurs when singing together, performing in an ensemble, or improvising collectively, suggests that the music classroom holds within it the possibility of an optimal learning environment. (p. 239)

St. John studied how children in this study influenced each other's musical learning experience through collaboration, and to clarify what collaboration looks like in early childhood. She examined collaborative activities from six 75-minute videotaped sessions of a music class consisting of 12 children aged 4-5 years. The ten collaborative events she chose to focus on featured collective musical engagement by at least two children.

Four of the events were singing, four were movement-oriented, and two featured instrumental play. St. John's video analysis concentrated on three elements: (a) the children's cycle of exchanges (verbal, physical, and musical); (b) if and how these interactions facilitated the children's engagement; and (c) how the children's collaboration changed the music material, possibly intensifying their experience.

St. John analyzed the data based on a music education application of "flow theory (Custadero, 2002), searching for occurrences of flow. She then closely examined the details of their interactions and coded the behaviors as challenging, or transforming. "Challenging" meant that the students either challenged one another, or themselves, to different levels of interaction with the music and each other. "Transforming" meant the 
students used teacher-given material and revised it for new purposes. St John found that children personalized their learning through interactive play, through challenging and transforming behaviors, such as self-agency, self-correction, expansion, and extension. She found considerable support for the idea that children helped each other change and re-use material, as well as the idea that children naturally collaborate and use one another's strength to intensify the experience (scaffolding): "Ideas traveled around the community of learners culminating in a rich quality of experience that was the result of many contributed efforts" (p. 252).

As a result of her research, St. John advocates for teachers to provide temporal space for students to create collaborative interactions:

As agents of their own learning, children seek out who can best facilitate their understanding. Children use peers as a resource for scaffolding ideas, creativity and enjoyment. Their interactions scaffold the learning experience. Music activities that promote collective experiences and encourage collaborative endeavors lead to unimagined possibilities in growth, development and creativity for all involved. (p. 256)

\section{Collaboration in Student Chamber Music Groups}

Most of the extant research on student collaboration, outside the student composition studies discussed in the previous section, is in high school and university settings (Berg, 1997; Davidson \& Good, 2002; King, 2006), with little information on student collaboration in elementary and middle school music making.

Berg (1997) looked at two high school chamber ensembles in an qualitative case study. Over a period of five months, Berg observed 33 independent group rehearsals or coaching sessions. Using an ethnographic analysis of interaction procedure, she evaluated the videotapes of the rehearsals and conducted formal and informal interviews with the students. 
Examining verbal, non-verbal, and musical interaction, she found intersubjectivity was created through discussion and performance, and students took turn assisting one another through the Vygotskyian zone of proximal development (ZPD). Interestingly, Berg found that instances of intersubjectivity occurred most often when quartet members arrived at decisions on more objective matters — rhythm, tempo and articulation — rather than interpretation of phrasing, dynamics, and tone color. In some cases, she found quartet members seemed not to resolve differences, but to "settle" for a peer or a coach's suggested interpretation. Berg characterized this as a reluctance to engage in conflict and a premature end to consideration of all the musical options. She reported these as possible negative characteristics of peer collaboration, which seems to parallel the findings in the literature outside music education (e.g. Barron, 2000; Palincsar \& Herrenkohl, 2002; Kynigos \& Theodossopoulou, 2001).

Berg also found that non-musical, social participation structures stemming from students' roles in larger socio-cultural systems (the school orchestra, the high school, the community) also had an impact on students' ability to engage together in music-making. Berg recommends that future study include regular viewing and discussion of rehearsal video by each individual musician, in order to focus attention on the participants' voice and experience.

Davidson and Good (2002) found a strong relationship between social and musical factors when they studied a string quartet comprised of British university students. Three members were female; the second violinist was the only male and the only non-music major. All players were considered by their chamber music coach to be at roughly equal playing ability, although the second violinist was regarded by the coach as 
very slightly beneath the others. The quartet worked together for six months, rehearsing two times per week. They socialized together regularly and the first violinist and violist were roommates.

The players were videotaped for one rehearsal, one concert, and one informal meeting post-concert during which they watched and commented on a tape of their performance. During the three-hour post-concert meeting, the researchers conducted a semi-structured group interview. They asked the quartet broad questions about repertoire, rehearsal interpersonal dynamics, and group goals. They also asked specific questions oriented toward certain moments in the performance videotape, related to the group's alignment and coordination.

The researchers examined the data in two ways. First, they looked for evidence of broad socio-cultural and socio-emotional issues in the group interaction, such as gender roles and performance anxiety. Davidson and Good found issues of concern were performance anxiety, the quality of the performance, and interpersonal relationships between quartet members. Performance anxiety emerged in the post-performance interviews: the performers reported a high level of distraction due to nerves. This affected the quality of the performance. All performers were concerned about their ability to meet the technical demands of the piece.

The researchers characterize the non-musical personal dynamics within the group as "striking" (p. 192). For instance, video and interview evidence supports the idea that the quartet members adopted certain, fixed roles. The first violinist was technical advisor and supporter/encourager of the second violinist and violist. The cellist was usually silent, independent, and isolated. The violist spoke quietly, and usually only to the second 
violinist. The second violinist, the male member, dominated the group by joking and opinionated comments.

The second way researchers examined the data was in an analysis of the groups' moment-to-moment musical interactions. The group focused their rehearsals on tuning, entries and exits, expression, and dynamics. The researchers identify "constraints derived from performer idiosyncrasies" (p. 196) as a large theme affecting rehearsals. The first violinist tended to drag in tempo, and the others had to compensate frequently for this. The second violinist was given rhythmic space to play tricky passages - the others would slow down slightly to help him — but he was still highly and consistently critical of the first violinist's tempo.

The researchers found a second large theme in the performers' gestures. These aided in marking dynamics, entrances and exits. The gestures involved "directions of glance," arm and head movements, and "circular body sway" (p. 197).

The players never discussed issues of musical meaning, and the researchers theorized that this was because the group had only worked together for six months. They were working at a technical level, focusing on tuning and rhythm, most of the time.

This study also considered the works performed, their relative difficulty, and the interaction of the static score with the living presence of the performers. The researchers theorized that because the players are focused around a fixed score, the coordination of musical content and process will be more controllable and predictable than the socioemotional issues that affected the group's results. They also questioned whether a longerterm analysis would still reveal the group working on technique first and saving the musical decisions for a future date. For future study, the researchers point to the need for 
a theoretical framework to be developed which will encompass the sociocultural, emotional, and musical issues which seem to envelop this sort of small group collaboration: "the musical work (in the Western canon, at least) is not only a product of socio-cultural factors but is also influenced by the interpersonal dynamics of the musicians rehearsing and presenting the work" (p. 200).

King (2006) studied the assumption of team role identity in her work with three pre-formed university student quartets: woodwind, saxophone, and string. She videotaped each group for three, one-hour rehearsals over the course of a month. Then she conducted semi-structured interviews with each member of the group to gather his or her insights about the nature of the collaboration within the rehearsals. The groups performed short (3-5 minute) excerpts at the close of the study for two university music faculty members, to be evaluated on the university ensemble rubric. The assessment evaluated their ensemble and group communication skills as well as their musical competence and technique.

King sought to answer the following two research questions: What team roles are manifested by student musicians in quartet rehearsal, and how does team-role behavior impact upon group work, including rehearsal progress, group dynamics and performance? She transcribed each rehearsal and coded events to observe the team roles assumed by individuals, the group dynamics for the rehearsal, and the musical progress evidenced by the group during the course of the rehearsal.

In this study, the typical roles assumed by the musicians were deputy-leader, contributor, inquirer, fidget, joker, distracter and quiet one. The students would change roles during and between rehearsals, usually to compensate or adjust for other members' 
mood changes. King had hypothesized that quartets with regular, more fixed roles and leadership would display more consistent musical progress and have a better end result; indeed she found that the string quartet, which exhibited more stable team-role behavior, a focused rehearsal attitudes and better progress than the others, made better musical progress. For King, this points out the effect of leadership on non-leadership roles on the musical success of a group. She advocates that students receive better guidance and training on how to take and yield leadership within a collaborative ensemble:

The students sometimes faced the problem of 'what to do' to improve pieces besides running through them several times, and their relative lack of experience in working together in a small group context exposed issues of 'how to get on' effectively to enable goals to be achieved. These concerns highlight the need for students to obtain both further musical training and greater skills in social collaboration. (p. 280)

King's research is a call for both more experience and more training in musical collaboration in the years preceding university.

Summary. The literature on collaboration in music education is concentrated on small-group composition and chamber music, with little research on the sorts of collective engagement that might occur — spontaneously or encouraged — within the larger elementary school music classroom. St. John's (2006) study locates collective music engagement within the larger social community of the classroom; however her research takes place at the preschool level. Other research on collaboration examines the interactions of high school, college, and adult musicians.

The sociocultural, emotional and musical issues which plagued the groups in the above studies seem to be inevitable. How might a teacher intervene to refocus or guide students in changing role or social dynamics? The role of the teacher in the collaborative musical interactions of young children is likely to be influential, but is little-studied. 


\section{Conclusion}

Research on collaboration indicates many variables influence its power to facilitate learning, in groups of teachers as well as in groups of children. The research reviewed in this chapter situates collaboration as a mechanism which instigates learning as shared understanding, not learning defined as one individual's cognitive change. In this view learning occurs within the shared, negotiated space among members of a group.

The above take on collaboration helped me study collaboration within the CTSG in terms of how we tried to construct and maintain shared understanding. The dynamics of teacher collaboration documented in the literature helped me understand and interpret the events of the CTSG, as I compared our experiences with past research. These connections are made explicit in Chapter Five.

The dynamics of student collaboration provided a useful backdrop to our discussions within the CTSG. While I did not bring this past research into our meetings, my knowledge of it helped me in the analysis phase to understand our emerging definitions of collaboration in elementary music, and underpin our principles of collaboration in Chapter Six.

The social constructivist stance of this study was appropriate for helping me understand the mediated reality_-"what is collaboration?"-co-constructed by each member and then held in common by the group. Chapter Three explains how the study was conceptualized and carried out using social constructivist principles. 


\section{Chapter 3}

\section{THE CONSTRUCTION: METHODOLOGY}

In this chapter, I recount in detail how this study was constructed and carried out as a social constructivist inquiry. Again, the purpose of this study was to examine the experiences of three elementary music teachers and me, the researcher, involved in a collaborative teacher study group (CTSG) designed specifically to focus on student collaboration in elementary music classrooms. The research questions were:

1. How do the participants describe their experience in the CTSG?

2. How has the focus on collaboration in the CTSG changed the teaching practice of these teachers?

3. What can these music teachers tell other music educators about collaboration?

\section{Participants and Site}

Participants were chosen for the CTSG based on the principle of intensity sampling, which means finding "information-rich cases - cases from which one can learn a great deal about matters of importance and therefore worthy of in-depth study" (Patton, 2002). However Patton is careful to note that intensity sampling does not imply the use of extreme cases: "one seeks excellent or rich examples of the phenomenon of interest, but not highly unusual cases" (p. 234). 
In locating information-rich cases, I considered Barnes' (2000) description of the type of teacher who would benefit from and bring energy to this type of group:

Teacher inquiry sustains extraordinarily busy people who are hungry for intellectual exchange, for time to analyze what they are doing, to share ideas, observations, and articles, even as they balance an astonishing range of demands on their energies and talents. (Barnes, 2000, p. 42)

Seizing on Barnes' well-chosen word, sustains — which means provides with the necessities of life, or keeps going with emotional or moral support-I sought teachers who would not only enjoy the process of collaboration within the study group but who might be enriched, or indeed, kept going, through it. Through recommendations from colleagues and my own knowledge of the music teacher community, I identified three teachers in the upstate New York area: busy professionals who seem to consider the sharing of ideas and observations around teaching practice a necessity of life. These teachers might be considered somewhat atypical in the sense that they wanted to add weekly meetings to their already busy schedule. They are not unusual or extreme cases in any other sense. However, their teaching situations, outlooks, and commentary on music teaching and collaboration provide a rich, informative, and I believe, characteristic, look into the world of elementary music teaching.

The three elementary music teacher participants in this study have public school teaching jobs. I am the researcher, but yet, it was important to me that I be a participating member of the CTSG as well. My actions and perceptions as collaborative teacher study group facilitator are part of the data; for a social constructivist inquiry it is appropriate for me to take an insider's view when I reconstruct the stories and findings of our group. The participants are briefly introduced here; more details on each individual are found in Chapter Four. 
Marlene is a flutist and a teacher. She teaches at three elementary schools in an upstate New York urban school district, Ridgeland (all places have been given pseudonyms). In 2007-2008, she was a first-year teacher in Ridgeland. She has twelve years of experience in other states and in Puerto Rico. Marlene has taken all three levels of Kodàly certification and all three levels of Orff-Schulwerk certification. She also earned a master's degree in music education with a Kodàly focus. During this study, Marlene did not have her own classroom in any school and taught music from a traveling cart. When we began the study, she felt that teacher-student collaboration is difficult in her setting, due to the fact she is not at any one school long enough to develop easy, friendly relationships. However, she had a strong interest in establishing the collaboration and community necessary for the Orff-Schulwerk improvisatory arrangements she likes. Andrea is a violinist and a teacher in a wealthy suburb, Pleasantford, where she teaches music to grades one through five. This district is generally supposed to have some of the best schools in New York due to the consistently high national ranking of its high school, and the high standardized test scores at the other schools. Andrea has bachelors (2005) and master's degrees (2007) in music education. She is committed to teacher professional development; in fact, her master's thesis was written on the topic of successful mentoring and development of beginning music teachers. At the beginning of this study, Andrea said classroom management is her strength, but she wanted to include more aspects of student collaboration in her teaching. Andrea's presence in the group helped to connect all of us; she went to school with Karly, and she took her OrffSchulwerk coursework with Marlene. 
Karly is a harpist who obtained a dual degree in performance and education in 2005, and a master's degree in 2008. During this study she taught in Edgeview school district, which abuts Ridgeland. Edgeview has many urban school district characteristics. In 2007-2008, Karly taught kindergarten, first, and second grade general music in one elementary school. Karly was interested in the CTSG because she continually seeks ways to give her students independent musicianship. She felt that more student-to-student collaboration might facilitate their musical growth, and was interested in finding ways to incorporating more collaborative projects within her demanding schedule of ten half-hour classes per day.

Researcher. I have seven years full time elementary school teaching experience and am a doctoral candidate in music education. I am currently an assistant professor of music education in upstate New York. I teach general music methods courses, music technology courses, curriculum, and music education history and philosophy.

From 2002 to 2004, I worked in music teacher professional development for The California Arts Project (TCAP). I was a staff member at three Collaborative Design Institutes, two-week summer professional development for arts teachers centering around Understanding by Design unit planning (Wiggins \& McTighe, 2005). These were substantial development opportunities for music teachers, as they featured intensive curriculum design work during 80 hours in the summer, and three follow-up weekends during the school year. I also conducted professional development series in six school districts, which involved weekly meetings with a music teacher cohort over the course of one semester. Finally, I was also employed by TCAP to deliver one-day inservice workshops on music education curriculum planning. 
My work with TCAP was the beginning of my belief that teacher collaboration is at the root of much teacher learning and change in teaching practice. I became convinced of the value of extended, sustained professional development, versus the one-day workshop. This interest propelled me into graduate study in 2004. My long-standing concern with professional development and its possibilities has not waned; this interest, and my belief that collaboration results in deeper learning and understanding, made me a natural fit to conduct this study.

\section{The CTSG Community}

The CTSG met on Thursdays for two hours, after school. We had seven meetings between April 1 and June 11, 2008. More detail regarding the dates of this study is found in the Timeline and Procedures section.

Our meetings were organized according to a protocol from the "Looking At Student Work" (LASW) movement in teacher professional development (Allen \& Blythe, 2004; see also www.lasw.org). LASW centers around structured conversation about student work: “[c]ollegial conversation, particularly when guided by protocols, offers a learning environment with unique features, and therefore unique opportunities, for the growth of understanding and professional skill and judgment" (Allen \& Blythe, 2004, p. 26). The CTSG's work was structured according to my adaptation of the protocols from The facilitator's book of questions: Tools for looking together at student and teacher work (Allen \& Blythe, 2004). (See Appendices A and B for the protocols).

I wanted the CTSG to be a collaborative experience, with symmetry in range of action and power between all members. I needed to make it clear from the first meeting that I was merely the organizer, not the one with all the answers. I tried to equalize power 
by asking participants to take turns leading the protocol discussions. I intended to participate in the same ways as Marlene, Andrea, and Karly.

I thought I might contribute theoretical knowledge I have of student musical collaboration. However, I ended up bringing very little theoretical or research perspective to our meetings. The topic of collaboration generated fruitful dialogue, and our protocolbased discussions kept us grounded in aspects of collaboration that we could see on the videos. Later, when I used the data from meeting transcripts to reconstruct the participants' stories and our socially shared definitions of collaboration, I was glad I had not inserted other researchers' definitions of collaboration into our discussions. We were free to create our own definitions based on our own views.

I did intend to use my role of university outsider to provide a safe and encouraging community where experimentation is encouraged. There is evidence (e.g. Zeek, Foote, \& Walker, 2001) that interaction with university faculty is empowering to teachers, as a recognition that their viewpoints are valuable to knowledgeable others. I was able to establish an environment of appreciation of mutual views in the CTSG. I believe that Marlene, Andrea, and Karly felt in my organization and facilitation the right balance between CTSG co-member and helpful outsider.

CTSG meeting description, as planned. Our meetings lasted over two hours. I established the following outline as a general plan. We did deviate from this schedule but not in any significant ways.

1. First 30 minutes: have snacks and beverages, and informally chat. This was intended to establish an atmosphere of collegiality, and connection on 
a personal level. This time of social interaction and relaxation was be a break from the CTSG members' busy schedule.

2. Proceed with discussion, following the protocol for a Collaborative Conference or a Dilemma Consultancy (adapted from Allen \& Blythe, 2004, p. 90). (see Appendixes A and B)

3. Decide who will host the next meeting, agree on who will provide the student work for discussion, and who will facilitate the next meeting.

\section{Study Design}

This study is a social constructivist inquiry. According to Guba and Lincoln (2005) the researcher in social constructivist inquiry is best described as a "passionate participant...a facilitator of multivoice reconstruction" (p. 196): one who attempts to understand the nature of knowledge through "individual or collective reconstructions coalescing around consensus" (p. 194). Control, in this type of study, is shared between the inquirer and the participants, which makes it especially appropriate for a study of a collaborative, non-hierarchical group of teachers.

Patton (2002) defines social constructivist inquiry as research with "the emphasis on the socially constructed nature of reality" (p. 99). The foundational questions for a study of social constructivism are:

How have the people in this setting constructed reality? What are their reported perceptions, "truths," explanations, beliefs, and worldview? What are the consequences of their constructions for their behaviors, and for those with whom they interact? (p. 96)

This study teases out how, and in what ways, the participants experienced and were affected by the CTSG. To find this out, I attended to the perceptions and views of all participants. Each perception and view was (a) affected by the social interactions 
among participants; and (b) highly dependent on the individual context of each participant's lived experience. The phenomena of teacher collaboration, teacher learning, student collaboration, and student learning are not "facts." They can be best understood within the context in which they occur. Social constructivism allows researchers to recognize the social and personal aspects of learning, and how meaning is constructed and appropriated within the group.

The challenge in research from this perspective is how to study the language and artifacts from the CTSG in a way that enables me to claim that I understand this "appropriation process" on the part of others. Schwandt (2000) calls this one of the "perdurable issues" that dog constructivist inquiry: "how to define what 'understanding' actually means and how to justify claims 'to understand"' (p. 200).

The CTSG in this research project is an environment of collaboration, one which I have made every effort to understand jointly and severally. Each participant perceived her learning, and the learning of others - the fellow participants, and the studentsdifferently. The transcripts of our semi-structured interviews and meetings allowed me to deconstruct each participant's experience in the CTSG, and vicariously reconstruct an understanding of her particular and socially constructed reality. I used the evidence from our meetings to trace the changes that occurred over time in the CTSG participants' viewpoints and perceptions, regarding teacher learning, professional development, and collaboration between teachers and between students. I used their words to tell each participant's story, and to find the commonalities and differences between their experiences. 
I talked with the participants about my interpretations of what was learned in the CTSG meetings. We agreed that we ended up with a shared understanding about collaboration in elementary school music developed throughout the course of the study. I was able to let the shaping process unfold naturally, and document it as we worked together on creating knowledge of collaboration.

Pilot

After the study was approved I met with a group of four elementary music teachers who were not involved in this study to test the proposed structure of the CTSG meetings. The pilot participants and I took part in a discussion based on the protocols adapted for this study. Because I had slightly changed the protocols from the Allen and Blythe (2004) versions to better reflect a discussion of student musical work on video, I wanted to see how the discussion would go.

On the evening of March 25, 2008, the four teachers and I gathered in a room at the local university. I had pizza and sodas for them. Two teachers, Jessie and Trisha (pseudonyms) brought 10-minute videos from their elementary classrooms. They said their videos showed students collaborating in various projects. Two other local teachers, Brian and Carl, participated in the discussion, which I facilitated.

Jessie was the first presenter. I had asked her to try the "Fine-Tuning Protocol" which requires the presenting teacher to share the context of the video first, including any pertinent information about the class, the setting, the students, her objectives, and the task. Jessie told us this was a fifth grade drum circle, working on African drumming, chants, and percussion on two different tunes. 
The protocol calls next for the presenter to frame a focusing question for us to think about while we watch the video and give feedback on later. Jessie asked us to watch for how well the students were collaborating by "getting into a groove": monitoring the steadiness of their individual part while still listening and adjusting to be with the others, and if she was doing everything she could to support that goal. Next the group is to ask clarifying questions, but we did not have any so we moved into watching the video.

After the video, we silently prepared feedback. Warm feedback, according to the protocol, points to strengths in the work and how it meets the goals of the students or teacher. Cool feedback might be questions, and defines possible disconnects between the work and what the teacher said her goals were. Jessie was to sit silently, taking notes on the feedback, and in the next section, respond to the comments or questions she chose.

Throughout the protocol, the facilitator is to clarify what is being said if necessary, and continue to draw the attention of the group back to the presenting teacher's initial focusing questions. At the end, the facilitator is to tie up any unfinished dialogue, and lead a talk about the process — not the content—of the protocol conversation and how it supported teacher learning.

I noticed we had difficulty giving warm and cool feedback on how well Jessie's students were maintaining musical ensemble. We did not say much about what the students were doing. Instead much of our comments were suggestions for Jessie, such as, "you could play a metronome in the cowbell part" or "you should stop them about 30 seconds after they start to get off."

In the warm feedback category we did say a few things that we had noticed, like, "the students watching from the floor were practicing drumming on their knees" and "the 
students are enthusiastic." However, very little of our discussion centered on the student work. We were anxious to help Jessie fix her self-professed problem of students who do not listen, and rush ahead in an ensemble. We did not talk much about what the students were actually doing but in the cool feedback portion of the meeting, moved into problemsolving mode.

Jessie's response to the feedback was a litany of things she had already tried, many of which had just been suggested. When she responded with comments such as, "I tried playing the cowbell and they just played louder to be over it," Brent and Casey chimed in with empathy. Casey said, "Oh, I've got one class like that" and Brent said, "I had the same problem; using the agogo kind of helped, but some classes don't listen to that either."

As facilitator I tried to bring the discussion back to looking at the student work, but I was unsuccessful. I finally ended up writing everyone's suggestions down on a big piece of chart paper and giving it to Jessie. She seemed happy to get their ideas, but I was not happy with the way that we had not uncovered anything about student collaboration in our discussion. It seemed to me that framing our discussion with a question had immediately led us to try to answer that question. The protocol is oriented toward "fine tuning" but it was easy to switch gears into strategizing and problem-solving.

For Trisha's video we used the "collaborative consultancy" protocol. It calls for the group to watch the video with no pre-conceived impressions, and write down what we noticed. Trisha's video showed small group of fifth graders teaching one another songs on the electric keyboard. I wrote down everything that people said they saw on tape. The list included things such as, "I saw children working together non-verbally to 
demonstrate the notes on the keyboard," "I saw students learning the tune and immediately turning around to teach some one else," and "Students were working so hard to master the song."

We then wrote a series of questions for Trisha. Our questions were about the process of teaching the students to teach each other. We wanted to know how she structured the small groups, how they stayed productive, and what she felt students got out of the peer teaching. We speculated she had probably found students to be better teachers than her in this scenario, and we wanted to know how the students had learned to be such effective non-verbal modelers.

When Trisha got to respond, she answered many of our questions. We found out she has a system in which she teaches three students a song at recess, and those three are responsible for teaching three more, and so forth. She said by the end of the next class period, nearly everyone will know the song. She likes to videotape the students teaching each other and watch to see how they do it; hearing that made us want to go back and watch several of the groups again so we could see their verbal and non-verbal interactions. We did that, and marveled as we noticed more of students' unconventional strategies this time. For instance, we noticed how one girl went around to the wrong side of the piano and taught the tune upside down, pointing to the keys one-by-one that her pupil was to play. She was a real stickler for accuracy, which made us laugh.

I was happier with the level of discourse about collaboration in this protocol. I felt we were really able to uncover new and exciting things about the small groups, especially when we went back and watched for the second time. We gave Trisha no suggestions. All 
our conversation focused on what the students were doing, and how it was fun to have a window on students' collaboration in peer-teaching.

After it was over, I asked the teachers what they thought of the experience. They were all positive. Carl asked if I could start a CTSG on improvisation, because he would attend. Jessie said she had gotten great ideas, and it was fun to watch Trisha's class on videotape. Brian and Carl agreed, and everyone talked about how they would like to see more video from each other's teaching. I wondered how unusual it was that four teachers in the same metropolitan area, who all studied music education at the same university around the same time, had no idea how one another teaches. They knew a lot about one another's general philosophy of music education, but had few specifics about how these philosophies were enacted in class.

I asked the teachers which protocol they liked better and they unanimously said the collaborative consultancy protocol. Trisha said she enjoyed sitting back and hearing what everyone noticed on the video. She said, "I think if I told you what to watch for it would bias you." Brian said he loved the "emergent, constructivist aspects" of the discussion; everyone laughed at his words because they sounded so aligned with the way I sometimes talk about teacher learning. However, I believe Brian did appreciate the way the collaborative consultancy protocol allowed a fuller picture of student collaboration to come forth.

After the meeting, I went back and watched Jessie's video again. I wanted to know why we had made so many suggestions to her, and speculated that perhaps she had been more of a teacher presence on her video than Trisha. While this was slightly true, the difference was not striking. There was plenty of evidence in the students' actions to 
give us a hint about why they were rushing; I saw students looking away from one another, and students who were not close enough to the group, for example.

I did not want to give up on the fine-tuning protocol. I felt it would have value in the CTSG if a teacher wanted to have us look for something specific, and if I were able to facilitate the discussion in a way that would keep the talk on "fine-tuning" and not "how to teach better to avoid a certain problem." As it turned out, the CTSG teachers all felt definitively that the collaborative consultancy protocol facilitated more interesting, thought-provoking discussions.

\section{Sources of Data}

Semi-structured interviews. I planned to interview the participants once before, once during, and once after the course of the CTSG, based on Seidman's (2006) tripartite structure for phenomenological interviewing. However, after the first two interviews were held, I did not feel that a third individual interview would yield much new information on the meaning of this experience. In the course of the CTSG meetings, I realized that our understanding of collaboration was being constructed and added to at each meeting. At each of our meetings, Marlene, Andrea, and Karly were talking about what they learned from each other and what they had tried out in the classroom in the ensuing weeks. Because this was a social constructivist inquiry, I was most interested in what the collective convergence was around meanings of collaboration. In June, I decided to suspend the third interview, with the idea that I would come back to it if I needed more data in the fall.

I went back to Seidman (2006) to read what he recommends in the event the researcher feels a change in the three-interview structure is needed. He states that the 
point of the structure is to uncover how participants "make meaning of their experience. If the interview structure works to allow them to make sense to themselves as well as to the interviewer, then it has gone a long way toward validity" (p. 24). Seidman says an altered interview schedule is appropriate, providing participants are interviewed over a number of weeks, their comments placed in context, and connected with other participants' experiences, to “account for idiosyncratic days" (p. 24).

Rossman and Rallis (2003) state that while new data can always be generated and new discoveries can always be made, ongoing analysis of emerging patterns during data collection can signal the researcher that there is enough data: "At some point you become aware that you can make sense of the people or settings you have been studying-you can tell a coherent story about what you have learned... the strange has become familiar" (Rossman \& Rallis, 2003, p. 264). I felt that after two interviews, my knowledge of Marlene, Andrea, and Karly's stories was deep.

After meeting seven times with the CTSG and a certain intimacy was established as a result, I also felt that creating another interview scenario might taint the collaborative, sharing relationship we had created as a group. I was concerned that even a semi-structured interview or conversation would feel artificial. In the fall, personal communication with the participants, a series of member checks of the participant stories, and my evaluation that much of the data was confirming or even redundant, established to my satisfaction that I had made the right decision to stop after two interviews.

In this study, the first interview was designed to find out as much as possible about what Marlene, Andrea, and Karly think about professional development and collaboration. Their words helped me understand their history with and views about 
professional development and collaboration, both in terms of teacher and elementary school music student learning. I did not use many structured or preset interview questions, favoring instead open-ended questions that enabled me to build upon the participants' responses. However, representative questions for both dialogues appear in Appendix C.

The second interview in this study, conducted after the fourth meeting, allowed Marlene, Andrea, and Karly to reconstruct concrete details of their participation in the CTSG, and tell about any effect on their teaching practice or student achievement. In an effort to tease out the sociocultural nature of learning in the CTSG, I asked them questions about working in groups and how they perceive its effect on their own learning. I asked questions about how they view student collaboration in their classrooms, and how they perceive collaboration's effect on student learning.

Videorecords of CTSG meetings. Digital videotape was used to record the events of each meeting of the collaborative teacher study group. I used a Sony digital camcorder which I placed on an elevated tripod, capturing a wide angle view of our meetings. All seven meetings were transcribed, verbatim, within Microsoft Word. I made a note of laughter, and significant facial expressions or other non-verbal cues as they occurred. Artifacts from CTSG meetings. The protocols used to structure the study group's examination of student work had written components. Participants made written notes on their copy of the protocols as they watched the videos. Participants emailed me with comments or suggestions for subsequent meetings. All these written components were potential sources of data, however most of them were duplicative when considered alongside the transcripts of our meetings so I used them just to confirm words or 
statements as I coded the data from the interviews and transcripts. The exception was the

personal emails from participants: these provided new information which was used to add to or triangulate findings.

Participant-researcher log. I kept a log detailing the events of the CTSG meetings. The log included all types of communication I had with the participants, including emails. The participant- researcher log also contained meeting protocols and agendas, and my meeting notes. I did not code this log in the analysis procedure; rather I used it to confirm timeline and location details.

\section{Timeline and Procedures}

Institutional Review Board Approval. The University of Michigan IRB Behavioral Sciences reviewed this study and determined on March 21, 2008 that it is exempt from ongoing IRB review, because it is included within the following federal exemption category: research involving the use of educational tests (cognitive, diagnostic, aptitude, achievement), survey procedures, interview procedures or observation of public behavior.

Initial semi-structured interviews. Initial semi-structured interviews were individually conducted with all three participants the week of March 24, 2008. I digitally recorded all interviews using GarageBand software on my MacBook laptop, with a builtin microphone. See Appendix C for representative interview questions.

CTSG meetings. The CTSG meetings took place on Thursdays, from 4:00-6:00 pm. The dates of the meetings were April 4, April 24, May 1, May 9, May 22, May 29, and June 11, 2008. We initially met in a conference room at the local university; subsequent meetings were held at one another's homes. 
Midpoint interviews. The second individual semi-structured interviews were conducted the week of May 6,2008, around or immediately after our fourth CTSG meeting. I followed the same digital recording procedures.

Analysis

I began the analysis with transcription. Using Microsoft Word, I transcribed all three of the initial interviews myself, which helped me prepare for the midpoint interviews. Two graduate assistants (whom I paid personally) transcribed the three midpoint interviews. I transcribed the first two CTSG meetings, and the same graduate assistants transcribed the remaining five meetings. As I watched the video and read the transcripts, I reflected on the research questions.

Social constructivist inquiry requires a purposeful attendance to the participants' worldviews, particularly to the interactions through which these views are created, altered, or reinforced. Throughout the transcription process, my re-reading of the transcripts, and watching the videorecords, I was aware that my job was to be "a facilitator of multivoice reconstruction" (Guba \& Lincoln, 2005, p. 196). As researcher, I assumed a "posture of indwelling" (Maykut \& Morehouse, 1994, p. 25) to immerse myself in each participant's experience. I searched their words for data to help me recount each person's particular narrative. I looked for information that would let me appreciate how Marlene, Andrea, and Karly understood their world as teachers and learners. I wanted to see if individual processes of knowledge generation could be traced in their words, and interpret what, if any, changes occurred as a function of belonging to the CTSG. 
First I looked for details about how Marlene, Andrea, and Karly felt about their job, their collaboration with other teachers, their professional knowledge and learning, and student collaboration. These are the broad categories I used to organize their stories. I then analyzed their words for changes in how they described these perceptions: changes that could be attributed to the social creation and exchange of knowledge within the CTSG in the months of April, May, and June, 2008. I cross-checked the interview data with the meeting data, and I looked through the transcripts for attitudes and ideas that stayed the same, as well. This process helped me answer research questions one and two in Chapter Four: how do the participants describe their experience in the CTSG, and how has the focus on collaboration in the CTSG changed the teaching practice of these teachers?

For a deeper look into research question one, I searched the data for consensus as well as individual distinctions. For consensus, I looked for descriptions of the CTSG experience, in terms of the strands that crisscrossed statements made by all four of us in interviews and meetings. I found two large categories of agreement. First, the CTSG was a professional development experience that, for us, supported teacher learning and change in practice. Second, the CTSG was an experience that addressed our individual and varying degrees of isolation.

I also sought to find individual distinctions within the data: specific evidence that would help me characterize and re-tell each teacher's unique experience. Statements from the three teachers and myself nicely converge around the idea that the CTSG supported teacher learning, and connected us with other music teachers in needed and meaningful ways. But for each teacher, those two important qualities of the CTSG manifest 
themselves differently. Each participant speaks personally about the CTSG experience, describing how teacher learning and teacher connection affected her.

For research question three-What can these music teachers tell other music educators about collaboration?-I combed the data for evidence allowing me to trace, chronologically and thematically, definitions of collaboration as they were socially constructed by the CTSG. Our group's understanding of collaboration was an evolutionary process, as the definitions converged and diverged over the course of our meetings. I looked for consensus and disagreement throughout between participants; I looked for consensus and disagreement between interview and meeting data as well.

For a complete list of my coding categories, see Appendix D.

\section{Goodness Criteria}

Guba and Lincoln (2005) state that the authenticity in the retelling or reconstruction natural to a social constructivist inquiry is the source of its trustworthiness. They describe the construction of validity as an extended look at a study's "goodness criteria" (p. 199). For Guba and Lincoln, the level of validity in interpretation is determined by the following two questions: "Are we interpretively rigorous? Can our cocreated constructions be trusted to provide some purchase on some important phenomenon?” (p. 205, italics in original).

In this case, the phrase "cocreated constructions" is particularly appropriate as the participants' voices were my source for an authentic reconstruction and analysis of the events of the CTSG. For rigor in interpretation of this data, I turned to the work of Lather (2003) who offers four types of validity guidelines as "data credibility checks to protect our research and theory construction from our enthusiasms" (p.190). 
The first of Lather's guidelines is triangulation. This means using multiple data sources, methods, and theoretical schemes. She cautions researchers search the data for non-confirming patterns as well as convergences. In this study, the data were analyzed for recurring themes, and for what Lather calls "counter-patterns" (p. 198): notable discrepancies or disagreements with theory, assertion, or assumption. The triangulation of method is sturdy, as built into the research design are elements of phenomenology (participant observation, and the incorporation of individual participant voice from the meeting transcripts), interviewing as qualitative research (interviews), and textual analysis (collective participant voice, from meeting transcripts).

Triangulating multiple theoretical schemes was more difficult. As befits a social constructivist inquiry, I was most interested in the thoughts and perceptions of the participants as they were freely created within or affected by our group. The social constructivist viewpoint (e.g. Palincsar, 1998; Rogoff, 1990) is the primary theory on which this study is based. The social constructivist view situates learning as shared understanding, not learning defined as one individual's cognitive change. In this view our learning occurred within the shared, negotiated space of the CTSG.

I obtained additional theoretical support for the idea that learning occurs in groups, through interaction, from the literature about community and learning (see Chapter Two for a full accounting). These previous studies provided a framework for understanding the signs I unearthed: the evidence that Marlene, Andrea, Karly, and me were indeed learning from and with one another in the CTSG.

Lather's (2003) second guideline is construct validity, defined as a constant and reflexive examination of how a priori theory may be changed by the data. For Lather, 
this process is "illuminating and change-enhancing" (p. 191) and has its roots in emancipatory social theory: "a ceaseless confrontation with the experiences of people in their daily lives in order to stymie the tendency to theoretical imposition which is inherent in theoretically guided empirical work" (p. 191). In this study, I constantly compared theory — the ways learning has been made visible and defined in previous literature - and the way evidence of learning by the participants in this study emerged out of their language and actions.

The third of Lather's guidelines is face validity. Face validity is achieved through member checks, which Lather describes as recycling analytical categories and emerging conclusions back through the participants for refinement. In this study, because of the nature of the CTSG meetings, we together constructed meanings and definitions of collaboration. As I began to draw a broad-stroke picture of the importance and value of collaboration in both teacher and student groups, I clarified my ideas and insights with the other three participants. The participant read and checked their stories, and the analyses I made of the data in Chapters Five and Six.

The fourth of Lather's checks is catalytic validity. As Guba and Lincoln (2005) remind us, "reality" and "validity" are not absolute: they come from "community consensus regarding what is 'real,' what is useful, and what has meaning (especially meaning for action and further steps)" (p. 197). Validity as "catalyst" is assessed through the value of the findings for instigating action and further steps. Lather defines catalytic validity as "the degree to which the research process re-orients, focuses, and energizes participants...respondents gain self-understanding, and, ideally, self-determination through research participation" (p. 191). 
The participant stories in Chapter Four were a final check for catalytic validity. I searched the data to find out: Were Marlene, Andrea, and Karly energized to take further steps or action after this professional development? Did this study have value for these teachers, in terms of real impact on their teaching process? What was the degree of “ontological authenticity” (Rodwell \& Byers, 1997), verified by asking: "what happened to the participants as a result of their involvement? Was their consciousness raised to a higher level of sophistication?” (p. 117). I would answer yes: each of the participants in their own way cited a greater awareness of collaboration in the elementary classroom, a larger degree of comfort with facilitating collaboration, and an increased sense of her personal, professional knowledge and capability.

\section{Organization of Findings}

In this multi-voice analysis of the overall experience, I have organized the findings into three chapters. In Chapter Four, "Chronicles," I present findings for research questions one and two in terms of each participant's story. Each story is written in three parts, which are Part One: At the Beginning, Part Two: CTSG Midpoint, and Part Three: At the End of the CTSG.

Part One for each participant begins with a portrait of each participant called the Starting Point. Within Part One I detail the participant's viewpoints into four sections: (a) the participant's job; (b) the participant's collaboration with other teachers; (c) the participant's view of student collaboration; and (d) the participant's professional development experiences, followed by a brief summary.

Part Two for each participant contains the same four sections as the first. My interpretation of the overall differences between the two interviews follows, called 
Changing Views. Part Three is a discussion of the changes throughout the course of the CTSG, followed by a summary.

After answering research questions one and two in terms of each participant, I identified two broad themes that recurred across Marlene, Andrea, and Karly's experiences in the CTSG. The extent of findings within these two themes substantiated for me the necessity of further exploring research question one in its own chapter. In Chapter Five, "The CTSG," I relate the two themes in common to research question one, and draw connections with previous literature.

The first theme is how the CTSG was professional development supporting teacher learning and change in practice for these teachers. In this section, I begin with a discussion of the CTSG members' previous experience with professional development, followed by six elements of the CTSG that affected its capacity to be an effective professional development experience: (a) the collectively generated knowledge in the CTSG; (b) the lack of development of teachers' musical content knowledge; (c) the discussion of students' musical content knowledge; (d) the necessity of video; (e) the importance of the protocol in effecting change in teachers' analysis of practice; and (f) defining and observing student work, versus teacher work.

The second broad theme in common is how the CTSG was a form of professional development that combated isolation for these teachers. I present the unique challenges for isolated music teachers, how isolation affected the CTSG teachers, and how the CTSG was in part a remedy for this isolation.

In Chapter Six, "Collaboration," I present the findings for research question three: what can these teachers tell other music educators about collaboration? Chapter Six 
includes a summary of the definitions of collaboration socially constructed by the CTSG over the course of our meetings, followed by four vignettes which exemplify these definitions in action. These vignettes illustrate principles of collaboration, drawn from my interpretation of the CTSG definitions, and which I connect to past research. 


\section{Chapter 4}

\section{CHRONICLES: PARTICIPANT PROFILES}

At the heart of social constructivist inquiry is a purposeful attendance to the participants' worldviews, particularly to the generative processes through which these views are created, altered, or reinforced. Social constructivist research can center around identifying and tracing a group's collective generation of these views. For example, I document the evolution of our shared understanding of elementary school collaboration in Chapter Six, "Collaboration."

However, to fully tell the CTSG story, I needed to pay attention to its collective and individual dimensions. In addition to uncovering and telling about our group learning, I needed to know what it was like for each participant to experience the CTSG. Therefore, for this chapter, I examined the participants' verbal representations of their views individually to answer two of my research questions: How do the participants describe their experience in the CTSG? How has the focus on collaboration in the CTSG changed their teaching practice? (In Chapter Five, "The CTSG," common views and issues between participants are grouped together thematically.) As I am an empathetic insider who had lengthy conversations with the participants, and participated in the CTSG alongside them, I feel confident in chronicling their stories here. 


\section{Marlene's Story: Introduction}

This is my retelling of Marlene's experiences throughout the course of the CTSG experience. I have written it as a story in three parts to reflect the changes in Marlene's views and practice as they evolved over time.

Part One: At the Beginning is material drawn from a 90-minute semi-structured interview Marlene and I had in early March, 2008. I interpret the findings from this first interview to create a portrait of Marlene just before the CTSG meetings started. This section is called Marlene's Starting Point. Themes that arose in our first interview are divided into a four-section discussion: a) Marlene's job; b) collaboration with other teachers; c) student collaboration; and d) professional development, followed by a brief summary.

Part Two: CTSG Midpoint is a snapshot of Marlene's views and teaching situation after the CTSG met four times. Marlene and I had another 90-minute interview at that time, in mid-May, 2008. We talked about the same four large topics as at our first conversation; what emerged was my sense that Marlene's views were changing. I have divided this portion of the paper into the same four sections as Part One. My interpretation of the overall differences in Marlene between the two interviews follows, called Marlene's Changing Views.

Part Three: At the End of the CTSG is drawn from Marlene's remarks at the last few CTSG meetings. Themes that arose in these meetings did not divide into the same categories as in the previous two. Rather, this portion of Marlene's story relates her own descriptions of successful changes in her teaching practice, as well as her continued 
challenges. In a summary, I discuss these changes, concluding that parts of Marlene's teaching are outside the realm of the CTSG influence, and explain why. Marlene's Story, Part One: At The Beginning

Marlene's starting point. Marlene's views on collaboration as we began this project relate to her tumultuous teaching conditions, and the lack of consistency around teaching and learning inherent in her schedule. Her take on student collaboration centered on observable behaviors: cooperating with the teacher and fellow students, listening, doing one's part, or simply allowing others to learn. Her views on teacher collaboration were similarly straightforward. For Marlene, collaboration with other teachers involved cooperation in dealing with students, collegial niceties—-such as sharing equipment, a room, or ideas - and working together in professional ways. The next four sections illustrate these points.

Marlene's job: "It's not your dream, I'm pretty sure." Marlene is an experienced music educator I first met in late fall, 2007. She was in her first year of teaching in Ridgeland Unified, a large urban district, but she had previously taught elementary music for twelve years in other states and in her native Puerto Rico. She has taken all three levels of Orff-Schulwerk training and has a master's degree in music education, with a Kodàly focus.

Marlene is an itinerant music teacher in Ridgeland. She travels to three different schools weekly and teaches from a cart because there is no room at any of the schools to house her in a music classroom. At each of the three schools, there is a full-time music teacher on site who does have a classroom; Marlene's job is to teach the classes that cannot fit into the schedule of the full-time resident teacher. This makes for an irregular 
schedule. For example, at one school she has two third grade classes, a sixth grade, and two kindergarten classes. Some classes are taught once a week by Marlene, and once a week by the full-time resident teacher: a situation which decreases the consistency of the students' musical education.

Marlene said she feels most at home at School 100; she's there almost two full days per week. Her colleagues at 100 are helpful and friendly. She feels like a "second category music teacher" at the schools she visits intermittently throughout the week.

I go to my other school and people still don't know who I am: "Are you a sub?" It's hard. Even the kids you know well, they barely see you during the week. They seem like, "Oh you're not good enough to be in the room?" (interview one)

Marlene is stoic about her working conditions, but keeps her sense of humor. At the first CTSG meeting, she introduced herself as a K-6 music teacher. Karly got excited, because she is a K-2 teacher who wants a K-6 job: "That's cool, that's my dream... to see the progression, that is so cool" (Karly, CTSG meeting one).

I said Marlene teaches at three different schools. With great comic timing Marlene said drily, "It's not your dream, I'm pretty sure!” We laughed, knowing that what Karly meant was the dream of working in one school, having the same students for seven years.

Marlene is positive but realistic about the challenges of teaching in an urban environment:

Some people criticize the district, and the teachers. But you'd have to be here to understand that you get some students that are disrespectful and rude and don't want to do anything, and you have to deal with that. And then you have a classroom with 23 or 25 students and, okay, maybe only four or five are having some serious problem. But it can make it impossible for you to teach the other students. (interview one) 
Marlene said planning instruction for multiple grade levels, at three schoolseach with different materials, space constraints, and environments - made it difficult to stay organized and meet the needs of all students: "You cannot please everybody but then you still have to deal with everybody" (interview one).

In the first CTSG meeting, she added that lack of communication within the district, especially between teachers and specialists, kept her unaware of some students' specific special needs or IEP requirements. A self-contained autistic class is a particular challenge for Marlene: "my mentor came to this class one day and said 'forget it! I can't even say anything about this!' Three schools. An autistic class. It is just too much" (CTSG meeting one).

Marlene feels somewhat ill-equipped for parts of this teaching situation. Despite Marlene's education, expertise, and district mentoring, at our meeting she spoke poignantly of how much she still needs to learn:

I still feel like I don't know anything. I still see situations that I don't know how to handle because they are very tough kids. Today I found out one of my student's mothers was killed over the weekend. That happens a lot! Too much! I don't know, nobody tells you how to deal with that. (CTSG meeting one)

Collaboration with other teachers: "We don't get to sit down and share." When I asked Marlene to describe teacher collaboration, much of what she said related to collegiality. Marlene describes collaboration as a sort of helpful, friendly cooperation: a necessary quality when teachers work in close quarters, sharing equipment, storage space, materials, and even students.

She described her relationship with the full-time music teacher at School 100 as a model. It is relaxed and easy, despite the fact that their interactions happen haphazardly: 
Sometimes I drop in just to see what she's doing and watch her... we share things. She has books that I have used, I have scarves that she used, it's really nice that we get along so well...she doesn't mind if I take something, like if I borrow the sticks she'll say, 'oh, okay. I'll use something else.' It's not like that everywhere, I know. I'm quite aware of that! [laughs] So I feel lucky. (interview one)

Ideally, Marlene wants to help students by working with the classroom teacher on the needs of students: "[Classroom teachers] know the kids better...they know their parents better, and...some of them get involved with me and music and some of them don't" (interview one). She likes to incorporate ideas from the classroom teachers whenever possible and said she learns from them. However, despite her desire to work with classroom teachers, their interactions are strained, or nonexistent, because she doesn't have the time or opportunity to get to know them: "At [School 100], I have twelve classrooms that I teach. So I know those twelve teachers, at least I know them better. The other ones I barely know!" (interview one). With good humor, Marlene related an incident when professional collegiality was lacking:

I had a teacher who [laughs] treated me like another student! And finally I had to stand up for myself, and she threatened to call the principal and tell him that I was not doing my job, which wasn't a job I was supposed to be doing... I'm not a five-year-old, I know what I'm supposed to be doing here! It got to a point where I had to tell the principal that "I'm trying to be a professional here, and she's not helping." (interview one)

Marlene said each teacher portrays the importance of music to their students in different ways; these various approaches manifest themselves in the behavior of the students. One sixth grade teacher has become an ally because she stays in the classroom while Marlene teaches music. "She says, 'That's interesting and that's so much fun' and that has made a huge difference...their attitude, it's completely different...If the teacher acts like she doesn't care, it makes it worse. (interview one) 
Pushing into the classrooms to teach music in non-soundproof rooms is also problematic in the establishment of collegial relations:

They can hear everything, from one classroom to another. 'It's too noisy! We cannot bear it!' My first week here, I was so upset, because I had just moved, and in a new environment, with a new language, but I was so happy I was here, and that was the welcome. [laughs] (interview one)

Marlene's relationship with other music teachers at her schools is constrained by lack of time and opportunity: "We get five minutes in the morning. But we don't get to sit down and share. If we had our breaks, or planning time together, we could maybe sit down and talk about what we're doing" (interview one). The lack of coordination between teachers is especially troubling to Marlene because of classes they share. At the first CTSG meeting Marlene told us:

At two of my schools, one teacher has a class for one music class a week and I have the other 30 minutes per week. Same class, two teachers! This happens at two of my schools. We [teachers] never get to sit down and say, "okay, we are going to do this." (CTSG meeting one)

However, Marlene said the situation is improving. She also said that in a way she likes being itinerant: less responsibility for concerts, committee service, and school-wide choirs.

Marlene is an experienced teacher, but being new to Ridgeland, she was assigned a mentor music teacher. Marlene is grateful for the mentor's assistance navigating a job in a large, urban district: 'I don't know what I'm going to do next year, when I don't have her anymore. It [this district] is a whole different world, with different materials, and different standards, expectations, observations" (interview one).

Her mentor's view on strict classroom management created some conflict for Marlene throughout the CTSG experience. Marlene's mentor told her to be "really tough, 
really strong, you know, not let anything slip by, and I'm still working on that" (interview one). But a dissonance between Marlene's ideas for incorporating collaboration, and those of her mentor and administrators, was illustrated in an anecdote Marlene told at the second CTSG meeting:

The administrators expect order...She [principal] tells me, "I want to see classroom management and integration of language." If they walk in, they want to see a classroom that is in order: they're engaged, they're doing what they're supposed to be doing. I'm afraid they're going to walk in and they're going to find one group here, one group there with the scarves, and everybody's dancing, and they will think, "What is she doing, just playing? [laughs]" (CTSG meeting one)

Student Collaboration: "Collaborating with the teacher." In our first interview as well as at the first CTSG meeting, Marlene related specific challenges of working in Ridgeland. Many of these challenges revolved around getting her students to collaborate, which Marlene defined as "everybody doing something, even just letting other people listen, letting other people learn" (interview one).

She also questioned whether her students were getting all they should be out of their music education. The following statement was prompted by watching the video of Karly's second graders:

They [Karly's students on video] are doing a lot, for second grade?! I am not sure my second graders would be able to hear, the chords and improvise on it like that? You know? They are thinking a lot. They are singing! A lot of times, my students, they don't want to sing, they're afraid to sing, they make fun of each other, especially as they get older. (CTSG meeting one)

As an example of their recalcitrance, Marlene related an example of the musical taste of her third graders: "My third graders are like pre-adolescents. They're so cool: 'can you play some hip-hop?' things like that" (CTSG meeting one). She said they 
would rather play recorders than sing, but that recorder instruction sometimes made her students work against one another. She tries to help them couch criticism more tactfully :

I'm trying to tell them, "if someone is doing the wrong thing, show them how to do it right, don't point fingers, don't just say, 'it was you! It was him who played the wrong note! It was him who was blowing too hard.' Show them how to do it...you take all this time telling me that someone is doing something wrong, and it's a waste of time. We could be playing instead of arguing." (interview one)

Marlene told me she wanted her students to spend less time fighting, and criticizing. She wants her students to become: "compassionate, understanding, know that everyone makes mistakes. We're here to learn, to become better musicians, better students, better people...good citizens" (interview one).

I asked her, "What one thing could be changed to make that happen, if I had a magic wand to wave to transform something?" She laughed, and said, "talking when the teacher is talking or singing. That's a big one... for me, that's the hardest part. Because I have to stop and make them listen again, and I don't like to stop too much, I like to do!" (interview one).

She told me that a successful strategy for her is to just continue with the lesson. Her district mentor doesn't approve of that, and wants her to wait for quiet before recommencing instruction: "my mentor doesn't like it, but it works. Because she wants me to be really strict" (interview one).

Marlene reiterated her view on the difference between what music teachers need to do, and what administrators might want to see:

They're not familiar with what we do and so they compare us to the regular teachers and, you know, we have to move, we need to sing and play and be loud sometimes and so it's hard...getting them to understand how it's supposed to be like. Sometimes a little noise and chaos is okay. (CTSG meeting two). 
Marlene said for her, collaboration would involve "collaborating with the teacher" (interview one) by being patient, listening, and following directions: "Everyone should sing when it's time to sing, everyone should do what they are supposed to be doing. And then everybody can learn and you can be more successful"' (interview one).

In the second CTSG meeting, after watching the video of Andrea's extraordinarily well-behaved class, Marlene said:

They were so nice. They would do everything that you asked them to do. And when you stop talking they don't start talking among themselves, you know? It's like they wait for your instructions, um..., yeah, and you get it done. It's like what you're trying to get done, they get it done. (CTSG meeting two)

Then Marlene looked at me and said, "I'm scared about tomorrow" (CTSG meeting two). The next day I was scheduled to videotape Marlene's class for presentation to the CTSG on May 1, 2008, meeting three. Marlene knew that her students were not as overtly "nice" as Andrea's; she may have been nervous about what would be recorded on tape. Indeed, the next day when I was leaving the school after videotaping, Marlene made a side comment to me that she did not think the students were very "good," i.e. well-behaved, on the video. I noticed that Marlene had spent a good part of the class asking for cooperation, direction-following, and collaboration. She also told the students they needed to collaborate and help each other. My perception was that quite a few of the students were doing what she asked, but that the overall progress of the class was delayed by a few. I told Marlene she might be surprised when she watched the video; even if some in the class were not well-behaved, she could take note of the number of students who were participating positively. At our meeting, Marlene indicated this class was not unusual, in remarks such as, "Well, it's real. It's real," "That's my class," and "typical!" (Marlene, CTSG, meeting three). 
Marlene's professional development: "I want more." Marlene said her district

offers professional development, but she finds it lacking in quality and quantity:

"I would like to see more, because I look all the time to see what's coming, what they are offering, and it's not that much... One time things, an hour maybe!

Because they have different little workshops. I want more" (interview one).

Marlene's need for context-relevant professional development was apparent. She

said sometimes courses were advertised by her district as appropriate for music teachers, but were only marginally connected to music. Although most of her professional development is provided by Ridgeland and ostensibly appropriate for this local context, she still finds it lacking. She said she wants:

Things to do with the kids, I mean things to do with this population that we have here...things that keep them active, that keep them doing something, keep them busy...How to do the whole lesson, a lesson that is engaging, that has all the components it's supposed to have and that the principal is asking for. (interview one)

She said she goes to workshops and thinks, "I cannot try that. I cannot do that, my kids are not going to handle that." (interview one) She also said that some sessions offered by the district emphasized the need to buy certain materials, or lacked important follow-up resources necessary for classroom application:

Maybe the explanation that is given is not enough for you to remember, and then they are selling a video, so it's like, they want you to buy the video....then there was a guy, who did really good African drumming, and we said, "we need you to write down the process of how to teach it" and we never got that. So I'm not going to be able to do it. (interview one)

She also described professional development as being "bombarded with a lot of information" (interview two) and typically focusing on lessons that, albeit cute and creative, require undue preparation: 
One was about literacy and music, and she used all these books...also all these props, like a Twister game [laughs], and mittens to make little fishes for the rainbow fish story, and I said, oh yeah, before the winter's over I'm going to go to Wal-Mart, I'm going to buy 25 little mittens and I'm going to make fish - yeah right! (interview two)

Summary of Marlene's starting point. Marlene's job, even by music teacher standards, is unusually difficult. When I first talked to Marlene the portrait that emerged was one of a good teacher struggling in a challenging environment.

It seemed that Marlene was fairly pleased with the support systems in place in Ridgeland, and she was optimistic about the capacity for improvement. However I thought for Marlene to feel successful in her difficult assignment, she would need more substantial and site-specific help, however. Even if the CTSG turned out to be a powerful learning opportunity for Marlene, I wondered if Marlene's schedule and teaching environment would prohibit her implementation of new ideas.

\section{Marlene's Story, Part Two: CTSG Midpoint}

In our second conversation, I was actually amazed at the difference in Marlene's attitude about her students, her job, and her teacher knowledge. Marlene described a general sense of well-being brought about by being able to talk to the CTSG teachers. She was also able to pinpoint specific suggestions made by CTSG members, targeted toward her precise situation, that had been successfully implemented in her class.

Marlene's job: "It always feels better the next day." In a mid-May interview with Marlene, I asked her what she thought of the CTSG experience. She said, "Good. Good. It always feels better the next day." She said coming to the meetings is a "relief:"

You know, it's like wow! I can talk about this [teaching] and I can see what's good. The other teachers, you guys, make me see what's good in it. And not just focusing on, you know, how difficult it is to work in the city schools and all that. (interview two) 
I asked her what specifically about the meetings made her feel the best. She said, "It's about giving," and explained:

Nothing could prepare me for this, how it's about trying to help other people. Trying to help teachers, to give them ideas. It's a two-way street. You know, they give you ideas, you give them ideas, and you hope it becomes better, and you feel better about your profession. (interview two)

Marlene's idea, that the CTSG was about giving, seemed to indicate that in the six weeks or so since I had first spoken with her she had gained some confidence in her professional knowledge and in the fact that she has expertise to offer. She said, “Sharing's just giving, it makes you feel good. I don't mind giving someone my ideas of what I do. Do you know how many ideas I've got from other people? It's like, I think it's my turn, to give" (interview two).

Marlene said the meetings were "like therapy" (interview two). I asked her if that was because of the particular make up of our group, or because of the structure of the CTSG. What she told me underlined the importance of a simple concept: teacher talk.

Oh my goodness, I think it would be good for any group of teachers, even if they are not as understanding and as supportive as our group is right now. But it will be good for any teacher to get to talk, because, well, I know I barely get to talk to anyone. Teachers never get to talk! I think every teacher could benefit from just talking and you know, letting things out. (interview two)

Collaboration with teachers: "In this school, it's pretty good." The quote above highlights Marlene's continuing desire to talk to fellow teachers. The CTSG was giving Marlene one outlet for this need, but I was not sure that was enough. However I did not expect the CTSG could have any impact on Marlene's ability to achieve this dialogue with school personnel, other than to perhaps provide her a model for how teacher-teacher interactions could be. 
In our second conversation, I thought Marlene seemed more confident and positive about relationships with colleagues. She did not complain about problems with collegial relationships and said she wanted to stay at School 100, where things are good. Here again, when defining collaboration, she emphasized the importance of helpful colleagues who support her efforts:

In [School 100], now, it's pretty good. I get a lot of help; students are always ready for me when I come in, and I try to leave them ready for [the teacher]. Teachers are helpful, very helpful. There's one teacher who's staying in the class, actually two teachers who will stay during music even though it's their break. You know, I haven't had any problems in this school...I'm hoping I'm just here next year. (interview two)

In our second conversation, Marlene did not give me any clues that she might have expanded her definition of what teacher collaboration could be. Despite her professed happiness with the detailed talk about teaching practice in the CTSG, she still seemed like she would be content if collaboration at work just involved friendly sharing. She did not say that she had a need to reproduce the CTSG-type dialogue with teachers at school.

Student Collaboration: "I think they're getting it." Marlene said she thought about planning for student collaboration in the weeks in between CTSG meetings: "There were a couple of meetings when people asked, ‘so what are you going to work on next?' I think about that collaboration thing all the time, in planning, and in teaching, I'm telling them more: let's help each other" (interview two).

Marlene's remarks made me think the CTSG focus on collaboration had given her another lens through which to view her students: a lens through which enthusiastic discussion is not seen as negative. For example, Marlene said the CTSG enabled her to look at her students' chatter in a more positive light. I recalled that in our first 
conversation, Marlene said that if I had a magic wand, the most important thing she wanted was to get her students to stop talking. At that time, she mentioned a difference between her mentor's practice (insistence on silence) and her own practice (continuing with the lesson in the hopes that the extra talking would stop eventually). However she seemed dissatisfied with both alternatives, and bothered by having to talk loudly so she could be heard over the students' chatter. She said it was her biggest problem.

However, in this conversation, she framed the situation differently:

I was so afraid when I came [to Ridgeland]. And I was taught to be tough, be fastpaced, and never miss a beat. I was taught, when you stop playing you need to say something right away, and not let them start talking. Now, I know you don't want to them to talk and "dah dah dah" and make comments - but, if you're telling the kids what to do all the time and talking at them they will never learn how to lead! How to have the initiative to do things, how to have the creativity, and so, I guess, the talking helps! (interview two)

Marlene now discussed her students' lively discussion as a more desirable attribute.

When she described her students' newfound interest in working in self-facilitated groups, she said, "They get really excited! They think it's a competition! [laughs]" (interview two). She explained that while her students still wanted to know who, or which group, was "the best," there seemed to be more excitement inherent in the nature of a group effort: "It’s like, "everybody did their jobs!” You know, they did great, everybody did great, everybody won! I like hearing that" (interview two).

Also, in this conversation, Marlene was more positive about her ability to communicate to students the value of collaborating with the teacher:

Today when I had the second grade I told them, "we are helping each other, you and me. And you need to help each other, too." They're saying, "Oh, we are here to help Ms. P?" And I think they understand that better than just, "sit down, be quiet and listen" and telling them with an attitude! (interview two). 
In our first conversation, she expressed frustration with the competitive nature of her students who blamed each other for missed notes or squawks marring their performance. At that time, she said she would tell her students, "If it's wrong, show them how to do it right, tell them how to do it, don't spend all this time arguing" (interview one). In our May conversation, Marlene said she had tried recently to instigate a less directive, more collaborative effort between students to eliminate missed notes.

Instead of looking at the person next to you and telling him to play it different, it's, "what can you do to help that person not make the mistake? Can you let him watch you? Can you play it for him? Can he play it for you?" (interview two)

I asked her if her students were learning how to collaborate. She replied, "I think they're getting it, they're starting to get it. I think they will get it, eventually. It takes a long time. I'm just now getting it" (interview two). I asked her what she meant, by "getting it." She said:

Well, I was the kind of teacher that stood there and, [sing-song voice] "Look at the board," "Look at what we're doing," "Sing together now," "Play together now." But I never divided the class in groups! I never let them really work on their own in groups! I don't know why. And I have the Orff training, it makes sense to divide them in groups. But then there's the issue of the space. But, wherever I can do it, I've tried it. Like, I've tried it with the two second grades, I've tried it with the third grade, that you saw on video, And I'm planning to do the composition groups with the fifth and sixth. (interview two)

I thought perhaps she did not previously do group activities because of her fear that observers would not understand the attendant chaos. I said that she seemed happy and excited to try some small group collaboration now. Marlene said:

I am! It's great to have something else to think about, to concentrate on now. before I was all, "these kids are making faces, talking, whatever" but you made me realize that they were getting something. Even if they don't look like they are [laughs] ultimately they are understanding something, or processing this information. And eventually they do try, and they'll get it. Seeing the video, I can see, I cannot expect everybody to get everything at the same time, of course! They are different kinds of learners. (interview two) 
The CTSG as professional development: "Yeah, I can try that." Marlene said the suggestions from the CTSG teachers are more relevant to her classroom than those she might get from others who have not seen her teach. She said, "It's like that a-ha moment. Yeah, I can try that. And what Andrea told me, I did try, and it was great. It did work" (interview two).

I asked her specifically what suggestion Andrea offered. In response Marlene related this success story:

[Andrea] said that I could have them [third graders] teach each other the song on the recorder. And I didn't do it exactly the way she told me, but I adapted it. And they were in groups, which was what I was afraid of, and it worked! And they wanted to do more. Then there was a second grade that did it with the rhythmic composition. They wanted to do it again. It was, "We want to do this more." They asked for more! More working in groups together. (interview two)

I asked her, "How are the ideas presented at the CTSG differed from other

professional development?" She said "the suggestions presented are more real, and you can do it right away. You don't have to wait until you get the book or get the materials. It's what they [other teachers] are doing" (interview two).

The video played a large role in Marlene's understanding of her own teaching:

As I told you before, when I'm teaching, it's like I see chaos! It's like, "oh my God, this kid is not playing, this kid is talking, this one is making a face." And then, you made me realize that they were getting something. It's [the video's] the real thing. So you can see it. It's like, video doesn't lie! [laughs]. Oh my goodness, it's much better to see it, to see what really happened. (interview two)

The way we talked about the video was important to Marlene. She appreciated the way the collaborative consultancy protocol allowed us to talk about whatever we saw in the video. She seemed relieved that the protocol would not require 
her to pinpoint for solution just one of what she saw as many difficulties in her classroom.

I couldn't pick a problem. I couldn't choose. It's like, oh my goodness! Everything is happening. I couldn't think about who's playing, who's not playing, how can I make everybody participate. The kid who's making the faces, the funny faces and making everybody laugh, what can I do with him? There's a lot of problems going on, a lot of situations. So it's hard to pick one, and it's still very tough to look at the whole thing, and look at the lesson as a whole, so, but I think it's good. It's good that we observe it all, and then talk, and then suggest...it's complete, it's like it takes care of every part, every aspect. (interview one)

Marlene also indicated that she enjoyed and learned from watching what the students did on the videos that Karly and Andrea presented. In our second conversation, she confided that she was still amazed by the second graders in Karly's video, shown at the first CTSG meeting on April 4, 2008, who were vocally improvising over chord changes. In our second CTSG meeting, Marlene said that because she and Andrea had taken Orff-Schulwerk level one together, she was happy to see how an Orff lesson played out with students.

Marlene's changing views. The value she had initially placed on quiet, cooperative students had shifted. While her core value of collaboration —as a way to help the teacher, and to help others learn - remained the same, the way in which she saw her students embodying that value had expanded. She was now more willing to accept talking and competition as possibly necessary side effects of student learning processes.

She said seeing her students on video, through the eyes of her fellow CTSG teachers, had made her realize that not everyone needed to appear as if they were learning in the same way. Categorizing the ways collaboration might look in the classroom gave Marlene other vocabulary to analyze and describe what her students were doing: a way of 
looking at her students that went beyond their behavior and outward manifestations of attention and focus.

Marlene also spoke about her own professional knowledge in a more confident way. I was interested in the way she described the CTSG as being about giving. She appreciated the opportunity to not only talk, but to share, and to give ideas to others. In our second conversation, she talked about the CTSG in terms of its immediate relevance and application to teaching practice. She credited the CTSG format for changes she made in activities she did with her students and for changes in ways she saw her students.

\section{Marlene's Story, Part Three: At the End of the CTSG}

In the last meetings of the CTSG, Marlene told the group about new things she had tried in her teaching, and how she felt more at ease with new strategies. While she is more aware of students collaborating, she experiences continuing difficulties in implementing the learning environment she wants.

Success. At meeting four Marlene told us a success story about having her second and third graders write compositions in groups. This was an idea she had gotten from Andrea at our May 1 meeting.

They went into the groups and they took turns to do one measure each and then they had to practice it, after they wrote it, and perform it for everybody. And some of them did dances and motions with it. And then I tried it with third graders, the class you know [from the video]. They practiced their piece in groups, without their recorders, singing, and then with their recorders, and then they played for each other and the class. This worked so well and was so easy, I had time left over! And it was so nice, to just go around and help them, as groups. (Marlene, CTSG meeting four)

Marlene said her mentor saw her teach this lesson: "I warned her, I'm going to try something different, and I know she was wondering... She didn't interfere. She loved it. I 
was so excited about it. I told her about you guys. Thank you guys for inspiring me." (Marlene, CTSG meeting four).

We applauded and cheered. Marlene went on, "I should do this more often. Because I felt relaxed. To be all the time in charge? All the time trying to control what happens? It's too stressful. So it was somewhat of a break for me" (Marlene, CTSG, meeting four).

To the May 28, 2008 CTSG meeting (meeting six) Marlene brought a video of students doing a similar small group project: learning to read and write the notes to "Hot Cross Buns." (More details about this lesson are found in Chapter Six.) She told us:

I knew I wanted to something that's in groups... I decided I wanted to try and record it so you could see how it went. It's the first time I've done [this lesson] with a class so I was a little scared and it looked messy to me, but I wanted to use the group work idea. (Marlene, CTSG meeting six)

We observed that in this lesson, Marlene was less concerned with controlling student noise and behavior. We thought it looked like more learning was going on.

Karly described the video:

If [an administrator] walked in for one second they might be like, "What's going on? It's noisy in here!" but if they walked in for five minutes they would see meaningful learning. So things got a little messy but they needed to, otherwise they would not have learned. (Karly, CTSG meeting six)

Marlene's use of a more collaborative activity and her courage to face her fear of letting the students talk freely as they worked together were two relatively new developments. Marlene also told us about another milestone in this lesson. She said she felt good about this lesson because of the way she was able to use some of her Kodàly pedagogy:

Teaching reading. The staff, musical notes, CDEFG, and not just saying it, but singing it and listening to it: the relationship between the sounds. What the 
Kodàly is about method is about, so it's hard to do. It's very hard to do. I understand why [other teachers] don't do it. Even my mentor didn't want me to do it. I try to once in a while get something out there to them anyway. (Marlene, CTSG meeting six)

Trying activities that fit into her overall philosophy of teaching music, although out of her comfort zone, or possibly eyebrow-raising for her administrator or mentor, seemed to give Marlene confidence in her professional expertise. She spoke convincingly about how students needed the skills they got out of this lesson. When Karly asked her if she had the goal of producing competent, musically literate children, she replied:

If they want to be musicians, or even if they don't choose to be musicians, they should have the opportunity. They should have a music class that trains their ear and develops them as musicians. They give math and science class to all kids, not just future engineers and doctors! (Marlene, CTSG meeting six)

Another piece of change evidence came from our wrap-up meeting on June 11, 2008. Marlene said as she continued to use CTSG members' suggestions about elementary music collaboration, she felt more at ease. She read aloud from the handout I made, which said, "the more you use these strategies, the more comfortable students will be." She added, in her own words: “And yourself, the teacher! It's definitely new for me, and I'm trying to do it more, get used to collaboration, and get used to the chit-chat" (Marlene, CTSG wrap-up meeting).

Marlene said she was also more aware of student collaboration. She told us a story of an "invisible" child, who barely speaks, and doesn't ever want to go out on a limb to participate in music class. But in collaborative activities she believes he is more relaxed: he doesn't have to perform alone, and other children can help him more. She said when his class was working on a folk dance, she was afraid he would not come out of his shell enough to hold hands with a partner: "He did it. I was afraid that he would 
freak out, because there was a lot of turning, with different partners, but they [other students] collaborated with him. They helped him do what he had to do" (Marlene, CTSG wrap-up meeting).

Marlene also said she noticed kids were often interacting positively with each other even when their eyes were not on their teacher. "I try not to tell them to watch me, now. And you can see them looking around, some of them trying to help somebody else, so that's good" (Marlene, CTSG wrap-up meeting).

Continued difficulties. One area in which I did not see much change in Marlene's situation is in the amount of confidence she had in her ability to get students to cooperate. While she had great success with new techniques and lessons that let students interact and work together, she was still feeling a great deal of resistance from students who did not respect her or each other.

At the fifth meeting, May 22, 2008, Marlene told us about trouble she was continuing to have in getting students to trust her. Her comments reinforced my belief that Marlene's teaching situation - the itinerancy, teaching in a new culture and in a second language with little daily support from colleagues — could result in a lack of confidence in her abilities. Meaningful change in this situation seemed beyond the CTSG's capacity to affect.

"I think my kids still don't have a lot of trust, because of everything that's happened to them, maybe?" she said. "I feel like it has taken me longer than ever, this year. Finally, mine are understanding more, but you can tell yours really trust and understand you, Andrea. They don't ever question what you're doing" (Marlene, CTSG meeting five). 
Karly reassured Marlene that her students as well are very opinionated. Marlene said she wasn't just talking about opinionated students, but students who did not see her as having expert knowledge:

This week, one of the students was questioning me, a sixth grader. He was sure the right hand goes on top of the recorder. He waited until his regular teacher was gone, he had a substitute and I think it was a day he thought he could say anything. He was telling me, "You're wrong. I'm going to ask the other music teacher." I guess he thought, I'm the itinerant music teacher, I must not know anything, on top of that, I'm the one who speaks Spanish, so you know they don't believe me. He said, "I want to go ask the other teacher, now." (Marlene, CTSG meeting five)

We asked Marlene how she dealt with that. She said, "I don't know, it's not good."

Karly encouraged Marlene by telling her, "well, on days when you want to crylike me, today, five times!-you just remember there's a bigger thing going on, you've got to teach them those life skills before you can even get to the music" (Karly, CTSG meeting five). Marlene agreed, and said she would think about that.

At the CTSG wrap-up meeting on June 11, 2008, Marlene told us about a new problem. In using more small-group projects in music class she had run into the issue of students being rude and inflexible as they chose their groups. Students refused to work in certain groups, and the students who were rejected felt ostracized. She brought this up as a difficulty in having a collaborative classroom.

As we talked to her about this, Karly and Andrea gave her a number of coping strategies: have pre-assigned groups, create positive consequences for students who are friendly and caring, and let students work alone if they wanted. They then tried to tease out if this was a problem for children in other scenarios at the school, or only in Marlene's music room. 
Marlene said she felt the students who had trouble functioning socially in music had problems throughout the day: "There's one girl: no one likes her, kids talk about her, and they don't want her in their group! But I can understand why, she stands up, she's loud, she puts her hands on them and gets in their face" (Marlene, CTSG wrap-up meeting). She added, "I can only do so much in 30 minutes to change her."

Also, Marlene was stymied by not knowing the students well enough to head off potential group problems. She said, “I have so many students! I'm not so sure who’s going to work together well. I'm not sure I can know them that well. There's no time!'” (Marlene, CTSG wrap-up meeting).

\section{Summary of Marlene's Story}

While Marlene had success with several techniques and ideas garnered from the CTSG, the lack of regular encouragement and support inherent in her situation are obvious in her wrap-up meeting remarks. The amount of institutional support in place for teachers can drastically affect their ability to make meaningful change. Her comments made me think that without substantial practical assistance in implementation, Marlene might struggle in putting what she had learned in the CTSG into practice.

In Marlene's case, the constraints of her teaching context make it hard for her to rise above certain concerns. Her role in the district as first year bilingual teacher, working in a new culture, and itinerant, affects the way she is seen by colleagues and students and the way she daily carries out her job.

First, the way she is seen by students and colleagues presents a constant tension. This is partially due to language and cultural differences, and partially due to the fact that she is unable to spend enough time at any one school to build the solid relationships that 
will preclude misunderstandings and breed respect. At least four times, she referred to students seeing her as not being "the real teacher." Marlene feels students need a relationship with her characterized by mutual respect and trust. However she says the small amount of time she has to get to know each student makes such a relationship almost impossible.

A number of times she mentioned things her mentor, colleagues and administrators wanted her to do, everything from not teaching Kodàly methods, to keeping children quiet. It is no wonder that Marlene's teaching expertise and instincts were finding little outlet in these circumstances, as she was frequently asked to carry out teaching that she was not wholeheartedly in favor of. The CTSG was influential in giving Marlene more confidence to institute some teaching methods she wanted to try, but without people on-site to reinforce the value of her ideas, it is hard to imagine whether these methods will become a permanent part of her teaching.

Second, Marlene's strengths as bilingual speaker and bicultural mediator are underutilized, and contribute to how she feels she is seen by students. She told the CTSG that she is the most comfortable in bilingual classes, because she can speak Spanish freely. She said, "Sometimes when I'm in an all-English speaking class and [the word] doesn't come to my mind, I look like I'm stumbling" (Marlene, CTSG meeting six). She also attributed some of her teaching success with collaborative activities to the fact she tried them with a bilingual class, "which means I feel more comfortable because I feel like I understand [those students] better. I know where they come from. Most of them are Puerto Rican. It's amazing, I didn't know it was going to be like that!" (Marlene, CTSG meeting six). 
She has a number of ambitious goals to meet, perhaps the biggest being her desire to know enough about the personalities and needs of her many students to be able to differentiate instruction effectively. Without opportunities to plan thoughtfully, consult with and get respect from colleagues, and reflect on how to create functional, collaborative learning environments for various groups of students, it is possible that Marlene will have trouble sustaining some of what she learned in the CTSG about establishing collaboration in elementary music.

At our last meeting Marlene said she did not think she or any other music teacher would ever want to stay at certain schools, "and not just because of the types of problems the kids have. It's because you don't get support to deal with what you get" (Marlene, CTSG wrap-up meeting). This frank assessment of one reason for music teacher attrition points to the need for more substantial assistance than a CTSG can provide.

However, Marlene experienced success in facilitating collaboration in ways suggested by CTSG members. The enthusiasm with which we greeted her positive story about collaboration at our fourth meeting encouraged her not only to try other collaborative projects, but to film one and submit it for our analysis at meeting six.

Marlene was even able to laugh about a foible caught on camera in her meeting six video. The fact that she was able to maintain composure and improvise during a complicated lesson — paired with an enticing student distraction—says a lot about her ease and confidence.

First she said that it was remarkable to watch the video with other teachers and see what we noticed: “I don't at the time see the things you see! It's like I feel blind, even 
though I'm right there! But did you hear when someone was yelling, "I'm bleeding! I'm bleeding!" (Marlene, CTSG meeting six).

She explained that her last minute preparation, made anxious by her mentor standing there asking about the lesson, led her to accidentally grab a pack of red permanent markers for students to write with. The extra degree of smudginess and the staying power of the blurred marks on fingers and hands gave the students a lot to exclaim about as some of them smeared "blood' on themselves accidentally-on-purpose.

She told us at that meeting:

I was in the flow. Ideas were just coming to me and I was just going with them, flowing with the energy. It's a lot less stressful, and if it didn't work, well, I made that mistake and then okay, what did I learn? A lot! But at the least, don't ever bring permanent markers to class. (Marlene, CTSG meeting six).

\section{Connection to Andrea's Story}

Marlene's story depicts the efforts of a teacher who wants to make changes in her situation, but is stymied by elements outside her control. However, the CTSG did give Marlene new ideas, confidence, and energy. Andrea's story depicts a teacher who got something different out of the CTSG experience. Whereas Marlene got new ideas, courage, and positive reinforcement for her efforts, Andrea got a new, more analytical and reflective way to look at her teaching and a wider perspective on learning.

\section{Andrea's Story: Introduction}

This is the story of Andrea, a participant in the CTSG. I have written it as a story in three parts to reflect changes in Andrea's views and practice over the time she was involved with the CTSG.

Part One: At the Beginning is material drawn from a 90-minute interview Andrea and I had in early March 2008. I interpret the findings from this first interview in a 
portrait of Andrea at the time we began meeting as a group. This section is called Andrea's Starting Point. Themes that arose in that interview are divided here into a foursection discussion: a) Andrea's job; b) collaboration with other teachers; c) student collaboration; and d) Andrea's professional development needs, followed by a brief summary.

Part Two: CTSG Midpoint is a snapshot of Andrea's views and teaching situation after the CTSG met four times. Andrea and I had another 90-minute interview at that time. We talked about the same four large topics as at our first interview; what emerged was my sense that Andrea's views on collaboration had expanded. I have divided this portion of the paper into the same four sections as Part One. My interpretation of differences in Andrea's outlook between the two interviews follows, called Andrea's Changing Views.

Part Three: At the End of the CTSG is drawn from Andrea's remarks at the last few CTSG meetings. Themes that arose in these meetings did not divide neatly into the same categories as in the previous two. Rather, this portion of Andrea's story details how the CTSG helped deepen her knowledge of her teaching practice, by illuminating and defining some of the most admirable features of the learning environment she creates for students. Quotes from Andrea in this section depict her as a teacher who has a sophisticated grasp on collaboration in the elementary classroom, and has good ideas about how to help other teachers learn more about it.

Finally, this section concludes with my interpretation of Andrea's still unrealized desire to have a more collaborative relationship with her Pleasantford colleagues. The 
CTSG was a model, but in Andrea's eyes not a replacement, for collegial communities of teacher-learners structured within one district.

Part One: At the Beginning

Andrea's starting point. In our first interview, Andrea seemed ready and excited to be able to share her thoughts about elementary student collaboration with Marlene and Karly, and to find out what they had to say about it. She said she envisioned collaboration looking different in each of their classrooms, and envisioned collaboration as a phenomenon that could exist in elementary music at a low or high-level.

Andrea was also enthusiastic about meeting and talking with us. Her previous overtures toward to other music teachers were for the most part unanswered. My sense was that her district's admirable efforts to strengthen teaching practice did little for the music department in terms of teacher learning or teacher collaboration.

Andrea's job: “They're Pleasantford kids." Andrea teaches K-5 music at one elementary school. This is her third year teaching, and her third year working for a wealthy, homogenous suburban school district situated in one of Ridgeland's most elite suburbs, Pleasantford. Andrea's school is enriched with all the extras one might suppose a highly funded elementary school would have: art, dance, theatre, integrated field trips, languages, clubs, and small class sizes. It is a very desirable place to work, by all accounts, and Andrea considers herself lucky to have landed a full time elementary music teaching job there right out of undergraduate school. Immediately after graduating with a bachelor's degree in music, she began a summer master's degree program at the same local university. She completed the master's degree in 2007. 
One thing that I noticed in talking with Andrea is the level of professional demands placed upon Pleasantford teachers. The demands may not be any more onerous than any other school district's, but different types of teaching improvement initiatives kept coming up in our first conversation.

For example, Andrea mentioned the "effective teaching" class that all new Pleasantford teachers are required to take for their professional development hours. It was not music-related, but she found it helpful nonetheless. Professional development hours are offered by her district and required for all teachers; one area for development targeted by the district this year is a new lesson plan format, which Andrea called "super intricate."

Another Pleasantford initiative for teacher development is the "target." Andrea and her principal devised three goals for her teaching; her observations focus on these targets for improvement. Andrea is conscientious about meeting her targets. One of them this year is "foster more student learning together," which she said dovetailed nicely with the focus on collaboration in the CTSG.

Andrea said one of her documented targets for last school year was "increase the love of music...to have fun, experience music, and just to love it: to enjoy coming to music class" (interview one). Increasing student enjoyment emerged for her as an important goal, because before she arrived, music class was perceived as boring mandatory work. Andrea worked hard last year to increase voluntary participation in chorus, to make the music classes engaging and fun, and to establish herself as a teacher with high standards who worked hard to create confident musicians.

Andrea said before she arrived: 
[Students] didn't learn anything. They didn't have any musical terminology at all, they didn't know how to dance, they didn't know how to move, even a simple game...Things they should know and be able to do, like moving to the music, walk to the beat. Oh, they could find the beat, but they weren't enjoying it! (interview one)

In Andrea's talk about the last two years and her attempts to build the program, she gave me the impression that her students were still not as advanced as they should be, which surprised me given Pleasantford's excellent reputation. However, other things she said made me wonder if that impression was completely accurate. For example, she told me this story of her favorite day in first grade music class:

We were learning so-mi, how to read it on the staff. Because they were doing so's and $m i$ 's and they had already learned $l a$, I decided to put a $l a$ up. I had it on the board and asked, "What do you think this is?" and Marcy sang it perfectly, with her handsigns and everything. I said, "Oh my goodness, my life is complete! They're finally getting it!" She was able to apply all this previous knowledge to a pattern she'd never seen before, and sing the pattern, and the handsigns, and all the other kids were like, "Oh, yeah, that's that pattern." And it was like, "click!" Everything worked perfectly.

I said, "Wow! That was a good day! Do you think the other kids learned more from seeing Marcy model her thinking?" She said Marcy pushed them harder to read the music: "It's nice to have those specific students in each class that really push everybody else to go a little further" (interview one).

I intuited from this exchange that Andrea had plenty of students who were highachieving. She said, "Sure. There are tons of kids who are eager to learn. They're always at the edge of their seats to read, to sing the song, or to learn the rhythm or do the instruments. And then the other kids in the class feed off of it! Which is nice! (interview one)

Besides the number of high-achieving, motivated students, another good thing about Andrea's job is her ability to connect with students. She knows all of the kids and 
many of their parents by name, and is aware of each student's general level of musical achievement. She also knows a great deal about students' non-musical academic needs from serving on the Instructional Support Team, which meets weekly to address the needs of struggling students. This role gives Andrea new and sometimes surprising information on students; she said she frequently gets the weekly list of the three students on the agenda and thinks, "wow! I never knew such-and-such was having trouble in the classroom, because when they're in the music room they're the star!" (interview one). Being able to be the bright spot in a student's academic life pleases Andrea, and she likes telling parents that music is an area in which their child excels.

As another positive thing about the school, Andrea pointed to the posters in her room defining five "Character Traits." She said the entire school works on traits, like respect, or responsibility, and holds monthly assemblies talking about those traits. She mentioned that her students transfer the knowledge about character into the music room; she likes being able to point out when students are caring about each other, being trustworthy, et cetera.

The overall picture I got from these remarks is of a school where caring staff members, parents, and administrators work together to improve teaching and learning of all students. Teachers in the building understand students and their individual needs. Teamwork ensures that no one falls through the cracks. Teachers work in conjunction with the administration to set mutual goals for improving instruction, and the students try hard:

They're Pleasantford kids. They want to please the teacher so most of them will go ahead and do it. Which is nice, I am not complaining at all! There are those one or two kids who are like, 'whatever', but they mostly just want to please you, which is just, you know, because it's Pleasantford. (Andrea, CTSG meeting one) 
Collaboration with other teachers: "What, here? No!" Andrea is not as glowing

about Pleasantford's opportunities for collaboration with other music or classroom teachers. I asked her if she is able to share ideas with the teachers in her building. She said:

What, here? No! The teachers don't bother. Like, I hate to say it, but music's just, and it's sad, viewed as a break time. I can't say it's all of them, because there are a couple of teachers who will come in the last five minutes to watch. I'll say to the others though, "We'd love to have you come in the last five minutes so you can see what we've done," and they'll say, "Oh, I'll see if I can come early." And they never do. (interview one)

I asked her if she ever attempted to integrate the music curriculum with the other subjects her students were learning. Given the holistic, child-centered view of educational community her school seems to value, I thought teachers would be willing to participate in connecting music with other subjects. But Andrea described a one-sided effort:

I try to find out from the younger kids, "what are you guys learning in the classroom right now?" And then I can find a song that's about penguins, or whatever. I try to do some of that cross-curricular thing, but it's hard. Just because, the teachers don't really support it. They think, honestly, music is a break time for them. Which it is, contractually. (interview one)

Andrea said occasionally the primary age teachers ask her to find songs on certain topics. However, it frustrates her that the teachers expect her to teach the song, once she finds it: "It's nothing like, 'we're doing a unit on Asia. Can you sing these songs, or can you give us songs that we can sing in our classroom?' They've never come to me and asked for songs for them to sing" (interview one).

Andrea told me that only once in three years has a teacher asked to trade lesson plans with her, so that they would both know what the other was doing. Andrea taught 
songs that would fit with this kindergarten teacher's curriculum, and the teacher sang the songs with the students all week. "That was a great example of how it works," Andrea said, "or, how it can work."

Andrea said that she wishes for more cross-curricular integration, but she sympathizes with the classroom teachers. She knows they are too busy to want to add musical integration to their job. However, in the first CTSG meeting on April 4, 2008, Andrea told a discouraging anecdote about the lack of support for integration.

Her principal had asked her to teach the entire school a song with lyrics reciting all 50 states, and their capitols, to a breakneck version of "Turkey in the Straw." She told the principal that she was "up to the challenge" but would need to recruit the classroom teachers to reinforce the words.

She said, "there was no way I could spend a month or more doing nothing but teach this song, which is what it would take, seeing each class two times a week." Consequently Andrea sent an email to the teachers, saying the principal wanted all students to learn the song so she would really appreciate it if the teachers would have their students sing with a recording, during transition times or as they walked to music.

I got so much heat for it! They [classroom teachers] said, "We're not going to spend any extra time on this!" I said, "It's five minutes. It's not even five minutes, it's two minutes, thirty-one seconds! Can you just have it playing as they come in, or as they leave?" They said no. But one first grade teacher, she totally played it with them, sang it with them, and they knew it so well...I liked it so much that she did that! (Andrea, CTSG meeting one)

After finding out about Andrea's relationship with the classroom teachers and their curricula, I asked Andrea about her collaboration with other music teachers in the district. She said: 
Music teachers? There's really no collaboration whatsoever. Our district is kind of sad that way. I think everybody just gets stuck in their own ways and they don't really care. Honestly, it's almost like they're burned out and they don't want to change. It's really sad. (interview one)

Andrea said that she and another relatively new teacher had tried to initiate more sharing among the elementary music teachers in the district, and got nowhere: "It was a "this is what I'm doing, this is what I'm always doing, I don't want to change' kinda thing" (interview one).

Andrea did say that a bright spot in her year was the Chorus Extravaganza: a week of joint rehearsals and a 400-voice concert with her chorus and another elementary chorus taught by her friend Tammy. She said:

We tried to include other teachers and they were so against it: "I don't have enough time." And they just put up such a fuss. Which sucks, because the kids, they loved it! To be part of it, to hear all those voices, was so much fun for them. Fun for us, to see their faces light up, and fun for them to show off. (interview one)

Her first year in Pleasantford, Andrea and Tammy worked very closely: "That was great collaboration...Tammy and I, we got along really well and we were able to talk about what we were teaching" (interview one).

Andrea explained that collaboration requires more than working at the same school or even sharing a room. "You have to be open-minded to do it" (interview one). She said she felt her efforts to collaborate with one colleague at her own school were squashed regularly: “the more I try, the more he's like, ehhhh [squashing sound in palm]. Anyway. Doesn't matter" (interview one).

I always assume that a reason music teachers don't get to collaborate with one another is because they're isolated, usually by being the only one in the building. 
I said, "So you can have someone nearby, and still not be able to collaborate, is what I'm getting from you?"

Andrea reiterated, "Yes. It's based on open-mindedness, and if your personalities don't get along, how can you collaborate?" (interview one). She added she does collaborate minimally with the band director in her building: they use the same language and posters for describing melodic patterns, so students can share common knowledge and vocabulary. Other than those two experiences, Andrea said she had no experience with music teacher collaboration.

Student collaboration: "There are different levels." I asked Andrea if she ever saw collaboration among her students. She told me a story about how she has a student who can never remember that the left hand goes on top when holding a recorder. She said the students in the class constantly whisper, in a kind way, "George, left hand goes on top!"

Interestingly, though, Andrea backed away from that story as an example of collaboration per se, by saying, "So that's a really cute little collaboration story, well, not collaboration really, but more like them just liking to work together, and care about each other. It's not really collaboration I guess" (interview one).

I told her that I did not have a set definition of collaboration in Andrea's classroom in mind: "I kind of wanted to get to what you thought of collaboration." Andrea rethought the last story and concluded, "Okay, then you could say that's collaboration." She added:

I guess to me, in my mind, collaboration is: they're playing together, or they're singing together in an ensemble, they're listening to each other. That to me is collaboration. I'm constantly asking, "Are you listening to someone who's singing next to you? Are you singing the same part? Oh no, you're not? Okay 
then how are the two parts matching together?" So, I guess when I think about it, I'm telling them how to collaborate and they do it. So I guess they're showing me that they collaborate, but I could say honestly I've never seen them do it on their own. (interview one)

She followed up her definition of collaboration by voicing the idea that perhaps collaborative activities fall along a continuum: "I mean, maybe there are different levels of collaboration. Like the lowest level is something as simple as, "George, left hand on top [laughs]".

I asked her to tell me more about the levels of collaboration. She told me about how her principal told her in her first observation of 2006 that she was doing too much in the classroom, and that she needed to let the students teach themselves more. She said she tried it, and the following year the principal said, "You're kind of doing it, but just try starting off with something as simple as 'turn to your next-door neighbor and tell them what you learned."'

Andrea said that in response to her principal's suggestions, she's been allowing more interaction: "I’ve been doing a lot more of that, pair up with a partner, or think-pairshare. Simple, low-low-low level collaboration. In my term's that's pretty low." I asked her for more examples of "low-level collaboration." She told me:

We were doing note names in third grade a couple weeks ago. I put six notes up and said, "Think of these names to yourself, and then find a partner and whisper to them and see if you got the same ones." That's a good way of quote-unquote collaboration, because sharing the answers, they get to talk about, "oh how do you know that's an A or a G?" (interview one).

I asked her how often her students talked and shared answers. She told me she has been making a point to include it in all her lessons. When I asked her if it worked, she laughed: 
They could totally not be talking about what you're asking them to talk about! But I trust them and they know I trust them. And I respect them and they know I respect them. So they're willing to do the work that I ask them to do. Honestly, I've never had a negative collaboration on that level. (interview one).

I asked her how she would advise another teacher to create that atmosphere of mutual trust and respect, so that productive collaboration might occur. She said the teacher needs to let go of some control, so that students can be corrected and therefore experience the limits of acceptable behavior.

I don't think the kids would feel comfortable collaborating with their classmates if the teacher was always, "Shhhh. No talking. No, stop. Stop talking." And then if she were to say, "Tell your next-door neighbor what you just learned," the kids would say, "are we supposed to talk now? Huh?" I don't think that they would be willing, I don't think they would feel comfortable, and I don't think they would really know what to do. (interview one)

I asked Andrea to describe her classroom environment, so I could better understand the atmosphere of respect and freedom within limits she was describing.

She brought the discussion back to mutual respect:

I'm not saying I'm a super strict teacher, but I'm not saying I'm a lax teacher. They know what my expectations are and they respect that. And they know I respect them as well...that's a huge, huge thing. And having the students care about each other is probably even more important... But having the kids know each other really well and be comfortable talking to the person they're next to, that's key. (interview one)

Andrea said she helps her students strive for a polished team effort: "I never let it go. If it's not good enough I say, I think you can do better, if you listen you can all end together, and have that nice sound at the end, working for that perfect ending." (interview one).

Andrea told me a story to explain how the "perfect ending" concept could lead to student collaboration. It was Hanukah, and the third graders were working on a 
complicated arrangement of a song with two xylophone parts, drums, rhythm sticks, and singing. "Thinking back to it, probably not a smart idea," Andrea laughed, recalling:

We were working on trying to get this perfect ending. And they just could not. They weren't listening, and the singing was "la la la" [makes tuneless rushing sounds] and ending was [pounds desk] in the drums, and it was just not working!

Andrea said she asked her students to come up with their own ideas for how to end together. She said they made funny and serious suggestions: "One of them was 'we could count 1-2-3-4' and we all stop', one was, 'let's say hi-ya, and one was 'how about the sticks people give us our cue to cut off?"”

Andrea said her students loved the last idea:

That ended up being their way to work together...it was because we had that nice, warm environment that they were willing to share. It was nice for them to come up with things themselves instead of me leading them... they had a discussion among themselves: "What could we do?" and "Let's try this." (interview one).

I asked Andrea if she would describe the above scenario as collaboration. She said, "Yeah! For them to be able to have a discussion for themselves, by themselves, without me having to say, No that won't work, try this. That was collaboration."

I said I sometimes hear teachers say that having students solve the problems is too timeconsuming. She said:

Time is valuable, that's for sure. But those are the learning moments I try for, for them to take ownership. I want them to take control. I want to teach, of course, I love teaching. But I also want them to teach themselves and say, 'Mom, guess what we did in music class today? I came up with a great idea to have us all cue together, so we could end perfectly!' I mean, that is something they will remember, hopefully, for a long, long while. (interview one)

In this talk with Andrea, I did not learn how she had established the respectful, congenial atmosphere she deems necessary for effective student collaboration. Andrea is still new in her career. She has only been full-time at the same school two years, and she 
admitted the first year was difficult. How did she develop such caring and respect among her students in a short time? I wondered how much of it was her, the students' eager-toplease attitude, or the overall school climate.

Andrea's professional development needs: New ideas that work. Out of the discussion of Andreas's views on collaboration came talk about professional development. I asked what she might learn about collaboration in a collaborative teacher study group:

It would be great to get some new ideas. I'm always open to different ways, but I'm kind of used to my ways of collaborating. It would be great to see how Marlene and Karly do it in their classrooms... "George your right hand's on top" is collaboration. Singing together is collaboration. Group work is collaboration. There's so many ways of classifying collaboration, so, I guess we'll get to see them in the videos. (interview one)

I asked her if her past experiences with professional development were positive.

She said, "it hasn't been great...most of the courses that are offered are really unrelated to music" (interview one). So she enrolls in any district offerings for the required six hours per year. She said the best professional development experiences she had had were interactive workshops she took for her own growth, not for district credit. Specifically, she mentioned the Saturday meetings of the Greater Ridgeland Orff-Shulwerk Association:

You get to try songs, actually play the parts so you really experience it. That helps your teaching. To experience it and to say, "this is fun for me and I know it's going to be fun for my students" rather than saying, "oh this song on this handout looks good" [pantomimes filing it away]. (interview one)

Andrea said professional development should be grade level- and subject-specific, "something that's meaningful to everybody so you're not just sitting there going, this doesn't apply to me" (interview one). She also said professional development should "let 
teachers talk about what we are doing in the classroom. What are we studying right now? What are our focuses? The more sharing you can do, you can always use new ideas, new outlook, different ideas" (interview one).

However, Andrea said the new ideas have to translate to her own practice:

That's how you know that you got something out of a course: you can take something you learned and put it into what you're teaching. If you're like, "that's a great idea," but you don't want to do it, then it doesn't mean anything to you! (interview one).

I said, "Do you think the CTSG will fulfill that need for sharing specific, applicable knowledge?" She said:

I think it will be really good just to talk to them [Marlene and Karly]. About what are they experiencing in the classroom, or what are the struggles that they're having. Just to have teachers be able to talk to each other and say, "Oh, I'm having a similar problem with my students. Wonder how we can come up with a way to solve both our problems." There isn't a place in my life, really, where I could sit down and say, 'I'm having trouble with this. What do you do about it?'” (interview one).

Summary of Andrea's starting point. At the beginning, Andrea had three fairly concrete views of collaboration in elementary music. One was that collaborative activities may be envisioned as falling along a continuum from low-level ("think-pair-share") to higher-level (students leading themselves and making independent decisions). Another was that a specific classroom environment—one of caring, respect, and secure expectations - should be in place for effective student collaboration to occur. The third was that Andrea said she tells her students how to collaborate, and gives them space to do it, but that she has never seen them collaborate on their own. I wondered if watching her own, and other, classrooms on videos would alter that conception.

Regarding teacher collaboration, I was saddened that Andrea has so little opportunity to work with the teachers in her building and district. She has initiated 
collaboration: she makes an effort to find music that aligns with what the students are learning, she created an annual joint concert with another school, and she has shared musical teaching ideas with other teachers who wanted to listen. She has a lack of support in these endeavors, however, and I wonder how long she can continue to optimistically ask for sharing opportunities from teachers who decline, before she stops asking.

I hoped that the CTSG would partially fulfill her need for teacher collaboration at this point in her career. Perhaps the structure of the CTSG would be effective professional development for Andrea, if it gave her the chance to talk realistically with open-minded teachers.

\section{Part Two: The CTSG Midpoint}

When I met Andrea in mid-May for another 90 minute conversation, the biggest changes I heard in the way Andrea talked about herself and her teaching involved an expanded awareness of student collaboration, and an expanded awareness of her own role in facilitating student collaboration. She felt the way the CTSG members examined specific elements of elementary music learning had given her a new way to examine her own teaching. Andrea also became more conscious of things that are positive about her job and students, knowledge that came through hearing how her own students and teaching looked to others.

Andrea's job. If one component of professional development is to improve job satisfaction, the CTSG worked for Andrea. Andrea was stunned by watching video from Marlene's classes: "It's mind blowing, how difficult her teaching situation is. It's opened 
up my mind and my thinking to say, 'wow, I'm so lucky, I have a room, I have great kids, I have instruments"' (interview two).

In an email Andrea sent me four days after our second meeting, she wrote she was gratified to see on video how cooperative her students were, something she had taken for granted: "I was honestly pleased with the way they work so well together and really are so well-behaved. I guess I didn't ever really stop to realize that” (Andrea, email correspondence, 4/29/08).

The CTSG meetings also helped Andrea see that communication with classroom teachers is not just a Pleasantford issue, it is a challenge for other music teachers. She made comments throughout the meetings that served to commiserate with Karly and Marlene's difficulties in this area. For example, at the May 1, 2008 meeting at which Andrea acted as facilitator, Marlene told us about how frustrated she was by not knowing which of her students had special needs and health problems. Andrea said, "Oh, that just opens up a whole can of worms. The communication between us and the classroom teachers is just like this [holds thumb and forefinger together]. It's so frustrating. I hate it" (Andrea, CTSG meeting three).

While nothing in Andrea's environment had changed, it is possible that she became more content in her position when the CTSG allowed her to step back and think about her teaching from an outsider perspective. She also may have felt support from the other music teachers in the CTSG who dealt with some of the same issues of colleague relationships.

The CTSG as Professional development: "Analyzing my teaching, and the 
results of my teaching. In our second conversation, Andrea praised the videos as being worth "a thousand words" when it came to making teaching situations available for group discussion. to Marlene and the way her videos gave a tangible look at teaching realities:

I had these images of my mind of what it was like to teach in the city school district. And it blew me away, just how serious her situation is, where she doesn't have a music room. She could have just said, "I teach on a cart and the kids are sitting around at their desks." And I would have been, "okay, I get it." But to see how those kids react to sitting in those chairs, versus being able to walk around the room, or do a dance...And she gets a ton of stuff out of her kids! (interview two)

I asked her if she had seen other teachers at work, either in person or on video.

She said that in her undergraduate music methods course, they had videotaped one another peer teaching, and that during her student teaching she had gone to visit Karly for a day, but never in her professional life. She gave another reason why the CTSG experience was different from those:

It wasn't in this detail, where we [CTSG] are actually looking to see what students are doing. This [the CTSG] gave us the chance to not say, "ok, Karly, you're doing this wrong." It's to say, "this kid's really getting it, this kid's working together." So we're watching but with a different focus than usually we do. (interview two)

I asked her if this was a different concept than most professional development. She said, "Definitely. 'Cause I think all professional development's all about teacher doing this, teacher doing this, teacher doing this." Andrea said the topics discussed in the CTSG are different than other workshops she had attended, which she also described as "all about the teacher:" "It's always about 'good questioning technique' or 'good classroom management.' It's always about 'what are we doing as teachers?' We never really talk about what the students are doing" (interview two). 
Andrea gave more evidence about how the CTSG is different than the workshop

above. She had been worried about that in the beginning:

I remember when you [Ann Marie] told me that we weren't going to be critiquing how we teach, that it was going to be talking about student collaboration, and I was like, "Uh, I don't know about that!" [laughs] You could probably tell I was kinda iffy on that. I thought, "how are three teachers not going to actually think about how Marlene teaches, or how Karly teaches, or how I teach?” But seriously, you were right. It has not come up in the sense of "What are you doing wrong?" or "What are you doing right?" It is more towards "what are the students doing?" (interview two).

Andrea said talking about the students' musical behaviors had been effective in

helping her learn about teaching. I asked her how we could possibly learn to improve our own instruction, if we were watching our students and not our teaching? I wanted to know if, and how, watching students on video had translated into a change in teaching practice or the musical achievement of students. Andrea said:

In the group, we're not talking about what I should be changing in my teaching. We're talking about "how are these students learning?" and that leads me into planning a little bit differently. So, we're not saying, "Andrea, you should be doing XYZ tomorrow." It's more like, "Oh, look at these students. They're doing this so well already, how can you extend this?" And that, in turn, affects my teaching. (interview two)

Andrea described how she assessed her students' musical achievement, and how that fostered analytical thinking about teaching:

It's not necessarily changes in my teaching, but my eyes are more open to what the students are doing, and noticing, "oh, they really are starting to interact" or "they're really working together in this way." More like analyzing my teaching, and the results of my teaching. (interview two)

I asked if analyzing her teaching was a form of professional development, and she used the word reflection:

Sort of like reflection, I think. I mean, that's developed me professionally. Going back to my lesson plans, seeing what I've done, realizing the steps I've done to 
get to this point, and just being able to observe more. I wouldn't have thought about it, had we not been talking.

One thing that Andrea liked about the CTSG is that we labeled some of the good things she was doing in organizing instruction. She said Karly had praised her "scaffolding" in the April 24 meeting:

There was a clear direction...let's start with the familiar and then we'll branch into the unfamiliar, and that's, they say that's a best practice, you know, building on what they know. From a teacher's point of view, that takes a lot of planning, and a lot of confidence within your own ability to keep the class going. (Karly, CTSG meeting two)

Andrea said, 'She gave the term, 'scaffolding.' I never really realized that's

what I was doing, going from class to class, year to year, and I never really gave that a term" (interview two). I asked her what she thought about her scaffolding process now, and if Karly labeling it had made her more conscious of it.

I was going through my lesson plans, planning for the next month, and, wow! I do it without knowing! And now I can name it: I am scaffolding. It's really cool! And I went back in my lesson plans, just to see, and I noticed where I brought this and this back from last year, or from earlier this year. So that's been really neat to see. (interview two)

Andrea also liked the protocol. She said, "I love this [collaborative consultancy] protocol. How much stuff did you guys get out of my teaching that I didn't even think about? Tons" (interview two). Andrea then described a situation where we might have been limited by defining a problem before the discussion:

If we had said, "In this video my students are rushing, I need you to help me solve it" we would have said, "These are the ways to solve it." But we wouldn't have noticed Colin for example, doing his shoulder motions to help his group ... You would really only focus on, "This student's the one that's rushing" as opposed to, "What are the students doing..." Maybe we wouldn't even have noticed it on the video, rushing, if I hadn't said anything. So I love that protocol. It broadens us just to talk about whatever we see. (interview two) 
Student collaboration: "I notice it a lot more now." Andrea said she was more

aware of what her students were doing. I asked her if she noticed evidence of

collaboration and she said she notices it more now. She gave me an example of what the

rest of the class was doing while two third graders played a recorder duet:

I was telling the kids to finger along while others were playing the song, so they could practice. I noticed that there were some kids that were grouping together, and they were just watching each others' fingers, and then one of the kids was pointing out, how to finger a specific note, and stuff. It's little stuff that I've noticed a bit more obviously. (interview two)

I asked Andrea what she did when she noticed this peer interaction. She laughed:

Before I probably would have said, "Are you talking when I'm talking? or when they're playing?" I've been more lax about, okay, if they're talking it could be because they actually are collaborating and it's not because they're goofing off. Maybe I should give them the benefit of the doubt! (interview two)

I laughed too, saying, "I've really seen on the videos that some of what we, as a teacher scanning around the room in the moment, think is off-task behavior..."

Andrea interrupted and finished my sentence: "Right! Actually is not! They're actually really learning and teaching each other" (interview two). She went on to say that collaboration is "constantly in the back of my brain...' oh, so-and-so's not just talking, they're talking about something that's musical, and they're interacting.' It's changed the way I observe my students, definitely" (interview two).

I asked if that constant observation and thinking had altered her definition of collaboration at all. She said, "I don't know if I would define it differently, but I see it differently" (interview two):

I still feel as though collaboration is when students are working together and they're singing together and they're listening to each other and all that stuff. But in my mind, before, collaboration could only look one way. Having watched the videos, I can see that collaboration can look completely different in Marlene's scenario and Karly's scenario and my scenario. It can be different, but it's still 
collaboration. So I think my definitions haven't changed, but examples of collaboration I think have changed in my mind. (interview two)

I asked her for an example of collaboration that may have "changed in her mind." She said, "what Karly said one time, that collaboration doesn’t necessarily have to be just within the students. It could be the students collaborating with the teacher as well. And I think that was like an "a-ha" moment" (interview two).

I reminded her that in our first conversation, she could not pinpoint a time when students collaborated on their own, without being asked to, or told how. She said:

It was happening before, probably, I think. But I think now because I'm aware of it and watching the students more, I try to almost make them do it! I create scenarios where it almost forces them to be collaborative without me telling them and without them really knowing it! (interview two)

She described a situation in which she tries to give them as many chances as possible just to interact, "because I think interactions, no matter what, increase learning" (interview two). She said for them to be able to teach, or to remind, another student that, say, "put down second finger for A" means they had to have that information in their brain, and then they had to solidify it by telling someone else.

At the CTSG midpoint, At the May 9, 2008 meeting, Marlene asked the group how students might learn to work together. Andrea said, "The more you do [collaboration], the more they're just, 'Okay, this is what happens.' That's my expectation, so therefore it's their expectation. You have to do it so much that they're just used to it that they don't question" (Andrea, CTSG meeting four). She was clear that these interactions were successful in her classroom because she expected the students to collaborate productively, and because they were accustomed to being asked. 
Teacher collaboration. I asked Andrea what it had been like collaborating with

other teachers. She immediately said, "I think it's been really, really fun" (interview two):

I loved hearing what Karly and Marlene had to say about the video. How much they got out of it was just phenomenal. And I liked being able to do the same for them, and then for them to say, "Wow, I totally didn't see that!" (interview two)

I asked her if it would be just as fun or productive with a different group of teachers, remembering what she had said in our first interview about how collaborating teachers needed to have compatible personalities. She said, "I think it would work with any personality, as long as they're willing to step back and not be nitpicky and very critical of the actual teachers." Then she reiterated how well the CTSG worked because "the fact that we're there to watch the students, just made it so much better." She summed it up by saying:

So I think that is probably the most important thing when putting a group together, is to make sure that it's clear that, "we're not critical of your teaching technique." It's more to see what we can learn from watching the students. (interview two)

She said if teachers were in a CTSG just to fulfill their professional development credits, they wouldn't get as much out of it. She contrasted that attitude with her perception of Karly and Marlene:

The fact that we were willing to do this and we wanted to do this, I think that's why we're so much more willing to really talk. So the attitude, or the reasoning behind why you're there I think has a lot to do with it too. (interview two)

She said the food, the relaxed atmosphere in one another's homes, and our comfortable interactions made the two-hour meeting length perfect, even from 4 to $6 \mathrm{pm}$ time after a full day teaching. She said that after four meetings, we are more comfortable and real: "now we're just more willing to speak what's on our minds and things like that, which is nice. We're much more open" (interview two). 
She volunteered, "I'm just glad that you asked me to do this. 'Cause this has been really fun. In the beginning, I was a little skeptical if we were really going to be able to not be critical of each other" (interview two). I asked her if the fact that we were not critical has stopped us from having meaningful conversations. She said:

Mmmm. I guess you could think that. But, it's not like we've held back in saying anything. I don't think we're being overly, like, polite. The question [on the protocol] is "what did you see?" You could go anywhere with that response. Like you could say, "I saw you messing up on that." You could go both ways. You could be really ultra-critical or you could just say, "that was good." But I think what's worked well is 'cause we were able to give specific feedback of what we did see, and that's helped us take that conversation further. (interview two)

Andrea's changing views. The biggest changes I heard in the way Andrea talked about herself and her teaching involved an expanded awareness of student collaboration, and an expanded awareness of her own role in facilitating student collaboration.

The CTSG experience gave Andrea new ways to look at and describe everyday phenomena in her classroom. One of these we labeled "scaffolding:" her ability to organize instruction and motivate students toward reaching higher levels of musical achievement. Another was the way students adopted her goals and worked toward them in a motivated way: we called that "buy-in." These phenomena are no less remarkable because they are commonplace in Andrea's classroom; rather, seeing them on video, working so well in a natural context, inspired the other CTSG members to try to recreate Andrea's learning environment in their own rooms. I believe Andrea was unaware of how exciting it would be for us to witness her helping the students in her classroom move step-by-step through progressively more difficult musical tasks and develop an understanding of ensemble. Hearing our positive comments about this process made 
Andrea more conscious of what she was doing well, and to strengthen aspects of this type of lesson.

Seeing the phenomena on tape also led Andrea to a more habitual reflective examination of her teaching and the learning environment she helps to create. After talking about scaffolding at a meeting, she noticed evidence of scaffolding student learning within her lesson planning. She said that she was able, even in-the-moment, to think less about her teaching, and more about learning, in an increasingly analytical way. She now watches for evidence of learning through collaboration; she talked about how she was able to give students opportunities to collaborate and have the powerful experience of achieving a musical goal in ensemble.

In our first interview she said that she had not seen students collaborating on their own. In our second interview she said that she was constantly thinking about collaboration, and noticing the potential of student interactions to be collaborative. She cited examples of student behavior that seemed collaborative; examples that she said she only noticed because of her newfound attention to the different ways collaboration might look in the classroom.

Part Three: At the End of the CTSG

At the end of the CTSG, three main themes arose that underscore Andrea's experience throughout. First, Andrea and the group continued our process of dissecting and understanding elements of Andrea's effective teaching: the sequencing and collaborative leadership we so admired. Second, Andrea continued to enlarge her awareness of collaboration, seeing it in new circumstances and environs. Last, despite the depth of Andrea's professional knowledge about music teaching and the impact she could 
have if able to share with other teachers in her district, Andrea continued to describe herself as not a full-fledged member of her school or district's learning team. The bounty of her knowledge, made so visible in the CTSG, was hidden to others who work so closely with her.

Unpacking Andrea's teaching. In the last few meetings of the CTSG, we extended the process of trying to understand specific elements that contributed to the collaborative character of Andrea's classroom. This effort helped us think about how we might incorporate some of the same elements in other contexts. It also had the effect of making Andrea more aware of what she and her students were doing. The video made teaching and learning more visible to all of us; the subsequent discussion refined our understanding.

On the video at the May 22, 2008 meeting, we saw an example of the way Andrea's expectations make it almost a given that her students will collaborate and work together. I said, "Andrea's got them totally buying in with her idea of 'the perfect ending"” (Ann Marie, CTSG meeting five). Karly described what she saw:

You've given them so many strategies. That's the cool thing: the ideas they're coming up with now, like, "Let's think it in our heads together. Let's mouth the words and think together" have obviously come from you in the past, but it's their idea now, on not just how to audiate but also on how to collaborate. You've scaffolded it for them... they're learning how to collaborate because you're modeling it for them, each and every step. (Karly, CTSG meeting five).

Andrea was amazed. She said:

This is amazing to see, what's on [students'] minds. When I'm doing this, I hope they're thinking the song, during the audiation time, but you don't really know if that will keep them together! The "thinking in their head together" thing, it really worked. It shows. Honestly, I never saw it this obviously that it works. Seeing the video: wow. (Andrea, CTSG meeting five). 
Marlene asked Andrea to tell us how she got the students to the point of internalizing ideas on how to collaborate. Andrea said, "I think of it as tiny hints: things that they might be thinking of, but they don't know they're thinking. But if I say it, they may think, "Oh, that's what I meant." That's how I help them process" (Andrea, CTSG meeting five).

At the same meeting, Andrea talked three times about how she wished she asked the students more questions. For example, she said: "seeing it on tape, I wish I had asked more to see what they noticed, because then they could give me feedback, on how it felt for them" (Andrea, CTSG meeting five). This is an example of how Andrea used the tape playback to learn more about student learning, and by extension, how she might change her teaching. She saw on the tape a missed opportunity to find out more about what students understood, and translated that into an idea she could incorporate into future teaching. Andrea reiterated the same idea at the last CTSG meeting: "Video's made me think a lot more about the students and what they're actually doing in class, since talking about it here, definitely" (Andrea, CTSG wrap-up meeting).

Range of collaboration. At the end of the CTSG, Andrea talked about her progress toward one of her school district-mandated learning goals: "foster more student learning together" (interview one). Marlene said what a relief she felt when her students were working together in small groups. Andrea agreed, saying, "You're not 'on' all the time." She added:

That's exactly what my principal said to me this year. He observed me and he said, "You need to let the kids teach themselves more." So that's why one of my targets this year was to do more collaboration, so this fit perfectly in. I step back: let them learn more. Instead of me always being up there and saying "You need to learn this and you need to learn this and this." There's a difference! (Andrea, CTSG wrap-up meeting). 
Andrea also said that focusing on collaboration in elementary music had given her a new way to teach. She said: "No one really ever mentioned how we could further than rote teaching, that we could actually go way beyond that into having the kids teach themselves. I've gotten that just this year!' (Andrea, CTSG wrap-up meeting).

I asked the CTSG how I could convey to other teachers in a workshop or clinic what they had learned. Andrea said:

In the workshop, let them experience it. Have the teachers themselves experience collaboration. Say, "Look! You might not have noticed it, but you were just collaborating!" And they'll say, "What? I didn't understand that was collaboration!"...Have the teachers themselves experience so they know how it feels. (Andrea, CTSG wrap-up meeting)

The idea of collaborative practices being placed along a continuum from low- to high-level emerged in Andrea's first interview with me, and in one of her comments at the last CTSG meeting. Here she talked about teachers' facilitation of collaboration as something that could occur in a wide range, although she cautioned that it was not an incremental process of development for teachers:

That's important for teachers to realize. It's not like, 'oh I've graduated from think-pair-share, I can't do that anymore with my class, I'm on to harder collaborative things"... It should be if you're, like we are, really comfortable with collaborating it's still okay to do a think-pair-share! (Andrea, CTSG wrap-up meeting)

The above quote is important because it illustrates Andrea's emerging view of herself as someone "really comfortable with collaborating." In contrast, at our March conversation, she had described her efforts at having students collaborate as "low level:" sharing answers, or talking to a partner. Here Andrea positions herself as a teacher with more expertise and an array of collaborative ideas to choose from. 
CTSG: not a substitute. I am certain that Andrea was gratified by her CTSG experience. However, the CTSG was not an effective substitute for Andrea's need to collaborate with Pleasantford colleagues.

In our first interview I was disappointed in Andrea's district. Pleasantford, while progressive and well-funded, does not seem to place a premium on music teacher collaboration, learning, or music integration. Andrea was not particularly glowing on the relationship between music and classroom teacher, either; early on, at the April 24, 2008 meeting, Andrea described some teachers as "great," versus others who she said "don't care, who don't show respect to me, and I know the kids pick up on that" (Andrea, CTSG, meeting two).

She said teachers at her school are caring and friendly, but do not to develop relationships that cross grade or subject boundaries: "It's not to say [teachers] don't talk, but I wouldn't see a first grade teacher going to the fourth grade wing without an ulterior motive...it's rare. It's not, "I hate you,' it's more like, 'I'd just rather sit with her.' " (Andrea, CTSG, meeting two).

At the last CTSG meeting I asked the teachers if they saw themselves as fullfledged elementary school teachers in the sense of being encouraged to work together and plan with others. They all shook their heads no, and Andrea explained, "There's no way we are even close to being able to work together like classroom teachers."

She then told an anecdote about how she requested that music classes not be scheduled on some Friday mornings, when the Instructional Support Team (IST) meets. Because she is a member of the IST, Andrea frequently has to get a substitute for Friday morning music classes and feels that those classes get shortchanged. 
I emailed the team leader and asked if I can really pack my Friday afternoons to have Friday mornings free on those weeks. She said, no, that I have to teach a fourth grade class on Friday morning so that all of the fourth grade teachers can have common planning time on Friday mornings! So perfect example: All of them get common planning time. I'm the only music teacher plus one traveling teacher and we never even get to talk! We're not given that time. Do they think we don't need it? (Andrea, CTSG wrap-up meeting)

Andrea said as great as the CTSG experience had been, she wished she could work on a similar project with teachers in her own district.

She said, "It's [the CTSG] beneficial but not as beneficial as if it was, say, a Pleasantford elementary music teachers group.” (Andrea, CTSG wrap-up meeting). This indicated to me that Andrea still has a need for meaningful discussions with her nearby colleagues to improve music education in her district.

\section{Summary of Andrea's Story}

The CTSG helped Andrea identify and understand some of the outstanding elements of her teaching. Our overwhelmingly positive responses to her videos probably gave her a boost, and we all benefited from the ability to dissect the collaborative practices in place in Andrea's classroom. In Fall 2008, Andrea sent me an email that reminded me how much she was still thinking about the CTSG:

CTSG has changed how I teach a lot - I am constantly looking for examples of collaboration during the class period, and I've changed how I teach things specifically to include more chances for students to work together. I've also tried to be less "strict" with what I do with the students, and let them teach themselves more (a la Karly). (Andrea, personal communication, September 2008).

Andrea's story to me is that of an outstanding teacher who has mastered many of the techniques she feels she needs to be an effective teacher, and who is now moving into a phase of her career in which she can be more reflective and critical. She is aware of 
many of the wonderful things she does to enhance her students' learning; the CTSG helped make her even more conscious of the positive outcomes of those efforts.

Andrea is not supported by other teachers in critically examining the teaching and learning of students in Pleasantford. Andrea is at a point in her teaching career when coordinated discussion and planning with other teachers would be mutually beneficial. She has much to offer beginning and veteran teachers in terms of creating positive collaborative learning environments; she has much to gain from further interview with colleagues who will continue to help her refine her ideas.

\section{Connection to Karly's Story}

The reflective, analytical quality with which Andrea was able to think about and discuss her teaching in the CTSG resonates with Karly's experience as well. Karly had many of the same frustrations with a lack of communication within her district as Andrea, however, some of Karly's frustration targets the music education profession in a larger sense. Karly presents her view of collaboration as quite different pre- and post-CTSG. One change is the expansion of her views of collaboration to include more social and emotional elements; in her story she is able to pinpoint what she learned from other CTSG members that helped to alter her views.

\section{Karly's Story: Introduction}

This is a story of Karly's experiences throughout the course of the CTSG. I have written it as a story in three parts to reflect the changes in Karly's views and practice as they may have evolved over her time of participation in the CTSG.

Part One: At the Beginning is material drawn from a 90-minute interview Karly and I had in early March, 2008. I interpret the findings from this first interview to create a 
portrait of Karly at the time the CTSG began meeting. This section is called Karly's Starting Point. Next, themes from our interview are divided into a four-section discussion: a) Karly's job; b) collaboration with other teachers; c) student collaboration; and d) professional development.

Part Two: CTSG Midpoint is a snapshot of Karly's views and teaching situation after the CTSG met four times. Karly and I had another 90-minute interview at that time, in mid-May. We talked about the same four large topics as at our first interview; I have divided this portion of the paper into the same four sections as Part One. My interpretation of the overall differences in Karly between the two interviews follows, called Karly's Changing Views.

Part Three: At the End of the CTSG is drawn from statements Karly made at the last few CTSG meetings. Themes that arose in these meetings did not divide into the same categories as in the previous two. Rather, this portion of Karly's story is a look at the way she expanded her definition of collaboration to include social and emotional facets, its role in assessment, and the need for careful sequencing for optimum results. At the end of the CTSG Karly also made some powerful statements about the group's role in helping her become a better teacher. In a summary, I discuss these changes, concluding Karly benefited greatly from the CTSG. Karly's need to have substantial serious talk about teaching had been met; also, she had taken ideas we discussed and applied them to her own teaching practice.

Part One: At the Beginning

When I had my first 90-minute with Karly in mid-March, 2008, I was impressed with her concrete examples of student music collaboration. She had considered 
everything from repertoire, sequencing, and room set-up in terms of its effect on collaboration, and defined collaboration in multifaceted ways that encompassed everything from whole-class to small-group.

Karly said it is important for students to collaborate because it stimulates their creativity and gives them a way to express themselves in partnership with others. Collaboration, to Karly, always involves a meaningful combination of musical ideas; she did not talk about collaboration in the terms of respect and caring I had heard from Marlene and Andrea. For her, music is the most important element of collaboration.

Likewise, Karly wants professional development that places musicianship at the forefront, and collaboration with teachers that enables honest appraisal of student musical achievement. She is aware that Edgeville teachers of other subject areas have rigorous standards in place, and they meet regularly to talk about student progress toward those standards. She would like the music department to attend to standards and benchmarks as well.

Karly's job: “We don't want to just sit back." Karly is in her third year teaching music. All three years have been spent at a K-2 elementary school in Edgeville, a large, suburban district just outside the city with a high proportion of English Language learners and a fairly low socioeconomic population. Karly’s job is fast-paced; some days she teaches ten classes of music in a row, and all her students are under age seven. Karly graduated with Andrea from the local university in 2005 and promptly got hired by Edgeville. She immediately started her master's degree in music education, working part time and in the summers; she completed it in May, 2008. 
Karly described many of her students as "very low: not all of them, but a lot of them, as far as their socio-economic base and everything" (interview one). She praised her colleagues, and said that one thing she had learned from the elementary teachers and administrators at her school is "they have systems that work. They are helping these kids."

To help students succeed, Karly said there are a number of initiatives at work: school-wide teams monitoring groups of students' progress, a school-wide focus on the integration of literacy across the curriculum, and support for teachers and administrators to meet and talk about interventions.

Karly appreciates the Edgeville structures in place that enable professional discourse among teachers. She values professional knowledge, wants to be a better teacher, and wants to help all her students. One way Karly helps her students is by serving on the School Improvement Team, which gives her a window into the strategic focus on English Language Acquisition (ELA) methods espoused by her school. Karly has adopted the school-wide push to incorporate literacy into all subjects in the building, adapting the concepts for music, because she believes in the school-wide language goals and methods:

It [literacy push] has nothing to do with music, obviously. And so what I've learned to do is to kind of flip that around, look at the positive side of that and say, "wow, they're doing this in ELA: are we really behind, in music? What active learning strategies are they using in ELA, and how can I incorporate that in music?" (interview one)

Karly has a positive attitude about translating these initiatives into music.

She said, "That's something I did on my own. I talk to a lot of special area teachers, and they say, 'oh this doesn't apply to me.' But you really have to make it your own” 
(interview one). She said she had tried to model classroom teachers' professionalism and create benchmarks, "just like they do. You know, 'my kids are at this benchmark, how do I get 'em to this benchmark'?" (interview one)

She admires the way the teachers in Edgeville pull together to reach common goals: "They meet together, and they work on specific strategies." She said she also admires the teacher collegiality in committees and meetings about helping the high-need population in Edgeville: “They have certain committees, like 'Looking at Student Work,' like 'Book Club,' all these things where they're coming together to say 'we want to help our kids out...we're going to help this kid"” (interview one).

Collaboration with other teachers: "I don't really have anyone to talk to. ” In our interview Karly expressed puzzlement and frustration about why there were formal mechanisms in place in other curricular areas, but not in music, for teachers to meet, assess instruction, and improve student musical achievement of. She said after she created benchmarks for her students' musical achievement, she realized she had no one to talk to about the realism and practicality of these goals, or how to help her students meet them: "I don't really have anyone to talk to about, them but I talk to myself! [laughs]" (interview one).

In our discussion, I referred to that feeling as isolation, but she corrected me: "Um, I'd say frustration. Frustration because I want to be the best teacher I can be" (interview one). She wants to be on a team that improves the education of students in all areas, but feels stymied at the way music is left out of these professional conversations. She said that the lack of common curricula and standard vocabulary made comparisons between music programs at different schools difficult. 
I asked her what she liked about the way other teachers in her building worked together, and if those methods could translate into working together with other music teachers in the Edgeville District.

"Well, the main thing that I really admire about them is that they have a set curriculum, first of all, and they're all dealing with the same curriculum. So the language is the same," she said.

“Apples to apples?” I asked.

"Exactly," she said. "And when you're comparing music programs, obviously, it's apples to, you know, grapes. Or candy! [laughs]".

She explained, "They're [classroom teachers] are able to discuss things in a very professional way, without any arrogance, and without any idea of, "my program is better than yours.' And I know we're not there yet [in music]" (interview one).

I asked Karly why, in a district like Edgeville that has mechanisms in place for teachers to consult with other teachers on non-musical curricular and student issues, should it be so different for music? She said attempts are made to discuss music curricular alignment, but when department meetings move from superficial topics to more important issues, things get uncomfortable:

It's been, "oh I have a cute idea!" "Oh, I have a cute idea too!" "Oh I made these really cute hearts, do you wanna see 'em?" And you know, when we finally talk about something great, we end up in a catfight, and so we keep them about "cute" (interview one).

I asked Karly to put aside for a moment the sorts of discussions she would like to have with other music teachers, and to tell me more about the teacher collaboration that does exist in her building. She said the "special areas" teachers (music, art, P.E.) at her school are "really, truly amazing...We want to really be the best we can be. We don't 
want to just sit back in our jobs" (interview one). At Karly's Edgeville school, they have ten minutes of "consult time" at the end of each day to talk with other teachers about student learning issues, and that is when she communicates with the other special area teachers:

I talk to the art teacher. I talk to the phys ed teacher, and we talk about students, their intellect, their growth, but we can't get specific. And that's the frustrating part: we can never get specific, like the other teachers do, and get to the nitty gritty! (interview one)

I clarified: “So, you're talking with them about general things, like behavior, larger issues of child development, things like, 'Johnny seems behind in motor skills.' But nothing about thinking in music?"

Karly said:

Yes. My team's great, they have some of the music vocabulary, they've learned from hearing my classes sing on morning announcements, and I've learned so much vocabulary from them. We're all willing to learn. But it's still not the same as talking to another music teacher. (interview one)

Karly displays an admirable desire to be a supportive team member, and she makes adaptations to school-wide improvement efforts so that they can be applied to music instruction. She chooses not to belong to MENC (The National Association for Music Education) in favor of the less-subject-specific NAEYC (National Association for Education of Young Children).

But Karly needs more connection with the classroom teachers. In the first CTSG meeting, she told us she has one class whose teacher sings with them everyday, and she notices a difference when they come to music: “They're already warmed up, they're in their head voices, they're ready to go. They're hearing stuff, they're thinking...the classes that sing a lot are always ahead" (CTSG meeting one). In the same meeting, Karly 
described her efforts to integrate music within the life of the school as one of her biggest challenges: "One of my struggles is thinking about how to talk to teachers about 'hey, how can we reinforce this in the classroom?"' (CTSG meeting one).

Karly's professional development: “We're not keeping up with the kids.” Karly has strong views on what she sees as our profession's failure to hold itself accountable to high standards. She is frustrated with the many in-service days and professional conferences she's attended; time spent in training that failed to equip her with the advanced skills and knowledge she needs to teach music better. Consequently, she feels our profession fails in its ability to help students go further, musically: "We're lacking skill, as a profession. Unfortunately, we are. We are not keeping up with these kids, and that's why we're not pushing them" (interview one.)

Also in our first conversation, she described a typical elementary school music workshop: "So many times it ends up as, 'This is so great! It's so cute! This is such a cute idea.' If I hear cute one more time, I'm gonna puke! [laughs]" (interview one). I asked her what would be an example of something important, and not merely "cute," that she would like to talk about, or learn, in a professional development setting. She said Edgeville had done a good thing last year in bringing in a nationally known music education expert, from the local university faculty, to give the teachers musicianship classes to build their own skills:

Starting there, once you have the musicianship in place, then you can really start having conversations about, "What content do I need to be teaching the kids? What is appropriate to teach these kids? You're doing what in kindergarten? How does that work? What does it look like? Why are you choosing to present a variety of tonalities, a variety of meters? What's your reasoning? And most importantly, how are you doing that, and how are they building that skill?" That's when you really start talking about curriculum. (interview one) 
I asked her if the musicianship classes had indeed resulted in having conversations like the one she depicted above. "That's just a very frustrating thing," she admitted, speculating that some teachers were afraid of exposing a lack of musicianship to their colleagues and therefore resisted the classes. "I think there's an element of fear and that gets worse as you get older...I don't even know that it's the case that they don't have the musical skills. A lot of them probably do, they probably improvise at home!"

Karly explained that transferring personal musicianship into classroom teaching of musicianship is a roadblock for some: "people love making music at home... then they get into the teaching setting and they're like [in robotic voice] "This is a whole note." And there's no connection. It's like they lost all the musicality!" (interview one) Karly is frustrated about the lack of common language and understanding of musicality in her district. She feels there is little meaningful talk about the content of music class: "There is just no conversation about music, speaking in music." She added, "Let me clarify: We talk about music. We talk about, 'That is a march. That is a this.' It's the musical part of music we don't talk about I guess. It's kept very separate," she said. She explained:

It's very easy to say to a teacher, "how do you teach kids about a march?" And they'll tell you. They have a million reasons how, and why, and they're very proud of those reasons... But there is not conversation about "how do we help kids really speak music?" (interview one)

I wondered how a conversation would go in which she would talk to someone about how to help kids really speak music. She said:

Ideally, it would be me going to another music teacher and asking, "My class is really ready for formal learning, and there's this whole class, ready, and Johnny is still not able to hear a cadential pattern" or something like that. "What other tools can I use? Give me some other tunes" or "What are you doing to bring them into 
formal learning?" Things like that. So, to me, ideally I would be on the phone talking about stuff like this every day! [laughs] (interview one)

I asked Karly what would make conversations with other music teachers as

productive and fulfilling as she clearly wants them to be. She said:

Basically finding out, what does the curriculum look like. The what, the when, the why and the how. "How do I enrich this experience [for students]? What does that look like in your building? How can we push each other to go to that next level?" And really challenge each other, I guess. (interview one)

I suspected Marlene, Andrea, and Karly hold different views of music education, its goals, and their priorities. I thought that the topic of collaboration would bind us together, and perhaps provide a neutral ground for discussion. I asked Karly, "What if another teacher had different goals from you? What would that conversation be like?" Karly answered:

That's a great, great point. One thing that I've really learned, in my youthful arrogance as a teacher [laughs] is that speaking musically about stuff is never a bad idea. Getting really set in your ways is a bad idea. So maybe, not using certain, loaded, terms? [laughs] Keeping things really open? And always basing the talk on actual work. Not saying things like, "my kids can do this!" but saying, "Okay, I tried this and it really worked, and here's what it looked like. And has anybody else tried that, and what did it look like?" When you approach things like that, you have smiles, as opposed to "grrr!" (interview one)

I was glad to hear Karly talk about basing discussion on actual work, as I had picked the "Looking at Student Work" protocol as a basis for keeping our meetings productive. I asked her what she thought the examination of classroom video would do for our meetings, especially considering her strong desire to have meaningful talk about music teaching.

"Video gives you a specific thing to talk about. You can say, 'okay, there, in the second minute, this was going on.' You can refer to it." Karly added she thought the protocol was a great idea to keep the group structured: "focusing on one specific aspect 
of teaching or content that probably someone else is thinking about, too. When you work together collaboratively you probably can touch on a lot of aspects that way" (interview one).

Student collaboration: “A meaningful combination of ideas.” In our first conversation, Karly expressed specific and tangible ideas about student musical collaboration. Her definition of collaboration was notable to me because she did not describe what the students did to collaborate. I thought she might define it as "students working together, helping each other, listening to each other" and so forth. Instead, Karly defined collaboration not as student behavior, but a product resulting from the process of collaboration: "A meaningful combination of ideas.".

She went on: "I've thought about this a lot! Because, when you first talked to me about this project, I was like, 'um, do I even have the kids collaborate?' I'd never stepped back and thought about it." She added, "It [collaboration] is necessary in music. There is no getting around it. It is 100 percent necessary, but it must be meaningful. It has to lead to some kind of heightened understanding, or some kind of meaning." I asked her if she could give me an example, and she told me the following story:

A little girl today, Angie, stood up and she said to the other kids, "Okay! You're on the drum, and you're going to be on piano today. And you're going to be doing this." And she said "One, two, three, four" and you heard "bam bam bam bam" [makes noisy, chaotic sounds]. But then I said, "Guys, are you interested in creating some kind of a group? Should we do something with this?" And they said, "Yeah! Is it a rock group?" I said, "Okay. Well look up there at our songs." - because we have a "Songs We Know" board for kindergarten. "Can we choose a song that is really special to you?" And they voted, and they picked a song. "All right, let's sing that song. Now, who has a solo they can take on that song? An improvisation? How should we do it? Should we start with the melody?" Now, that's the collaboration I want to happen. It comes from them. (interview one) 
I was amazed by this anecdote, although I knew it was authentic. My

undergraduate music methods students and I had witnessed exchanges just like this many times in Karly's classroom. The scene she described seemed to me to be collaborative at its core: student-centered, teacher-facilitated, enabling student self-expression and creativity as everyone worked together to make something musical.

She went on to describe the progression of student collaboration in first and second grades:

First grade...now we're having the conversation, "do you want to do this in duple or triple?" "We wanna do it in triple!" "Okay, well what's a good rhythm pattern for that?" "What's a good improvisation for that?" "How should we put it together, what instruments should we use?" Second grade, they go that extra level. They're really arranging tunes. (interview one)

I wanted to find out, if collaboration is a meaningful combination of ideas, what an un-meaningful combination of ideas would look or sound like. She laughed: "I don't want to get myself in trouble here! Maybe a non-musical activity, of acting out a story to a song?" She said she gives her students credit for hearing, and knowing, when they did something musical, "as opposed to just playing whatever" (interview one).

I wondered what happens in a classroom, realistically, when only some students might be meaningfully combining ideas and music with others. Karly said she does a lot of "whole group collaboration," which she described as a group activity in which "each kid has a meaningful role:"

Tina comes to me a couple days ago and says, "I've got this" and she sings "Amazing Grace" for me, gospel style. We learned it based on Elvis' recording, they've learned melody, bass line, improvised over it, some of them have learned the bass line on piano. So Tina takes the melody somewhere else, she personalized it. She told it to the class and they started singing the bass line with a rhythmic variation. Then we asked, "okay, who wants to do what?" They decided, "Okay, I'm going to take this leadership role." We even had a girl decide, "I'm gonna give the cut-off, here." What I do not want to happen is them sitting there 
and saying "I don't know what I'm doing." Some classes work better, like that, as a large group, but each kid has an important part. How do I as a teacher, without freaking out, say, "maybe they have a better idea than I do" and they usually do, honestly! It makes me a better musician, because, now I have to come up with a new piano part for their new version of Amazing Grace! (interview one)

In the first CTSG meeting, Karly brought a video of a second grade class working on an arrangement of "Amazing Grace." A comment she made about that video confirms her goal to involve every student in the collaboration. She said, "I would say $92 \%$ of them [students on video] feel really like it's theirs. Now my challenge is ... how can I get everyone really, really into that?" (CTSG meeting one). Karly has thought about managing a classroom when doing these large-group collaborations. She said the secret is to be structured from the beginning, and create a social contract together, with music class expectations written by the students. Even her contracts imply a collaborative spirit; I could see the contracts posted around the room, handwritten by children in their language, implying that students have most of the input.

She said her students understand that if they goof around, there will be a consequence. Her students also understand the positive consequences of trying hard and doing their appointed jobs: "if they're learning the bass line, and trying, they're gonna get on the piano a lot quicker, and I'm going to give them a leadership role. They will work toward that" (interview one)

She had more to say about getting the students to work collaboratively toward a goal:

Some classes are more successful than others, if I'm going to be honest. But as far as collaboration, the energy is there first of all, and they want to do it. They're talked to all day long... So the fact that they have some ownership, I find that a lot of the behavioral stuff goes away immediately because they're like, "I get to do what I want. I get to make a choice in here!" Sometimes they get to sign up for what part they want. (interview one) 
I asked her what happened when she was less successful, and she said, "as far as behavioral management, when I'm doing collaboration, there is no behavioral management. My problem is when I'm teaching." When I asked Karly what the difference between "doing collaboration" and "teaching" is, she said:

I mean, when I'm teaching, as in presenting. How am I going to keep their interest? I find that saying, "This is going to lead to this! This is the product we're looking for," really gets them energized. Otherwise they're, "why are we talking about duple meter?" But really putting it in the context of collaboration is key. They want to do it. And they'll listen, and they're ready, just because they want to do it. They just need to want to do it (interview one).

Karly's clear distinction between teaching-as-presenting, versus collaboration, is unusual. The presentation mode is a persistent model in our profession: not just in music. Many teachers are most comfortable in teaching-as-presenting.

"It's a fear," Karly said. "Once you give up power, you put it in the hands of the kids. And that's scary! They're smart!" Karly spoke about the power issue again, in terms of how professional development might help give teachers confidence to do more collaborative activities like she does:

I really do honestly think it's a power thing. Not that you want to be in power. It's just, "the class could fall apart, because I don't really know how to get the class to collaborate, I haven't had any professional development, I haven't had any training in that. I have no idea what collaboration looks like, and I'm a little too afraid to ask the person down the street because they might think my program sucks." (interview one)

She presented the other end of the spectrum as equally ineffective. "You can't ever give kids complete control. You're the teacher, you were hired! [laughs]." She described the successful classroom as being balanced between teaching new material and reinforcing previously learned material through collaborative projects. 
One of Karly's goals in music education is to render herself unnecessary. At the first CTSG meeting, she told us that at her spring concert she plans to be "completely out of the equation, it will be completely theirs...My goal for them is really to be fluent and independent musicians" (CTSG meeting one).

Karly has her classroom physically arranged in a unique way. There is a large amount of open space, decorated with circles that mark a space for each student. There is a taped-off area around the piano, a tiled area with xylophones and drums, and several small nooks elsewhere in the room labeled "Composers' Corner," "Music Club," "Secret Agent Club." I found out that each of the distinct areas of the room were intentionally arranged to serve a purpose; the overall plan has helped her establish freedom within boundaries:

This is kind of a dualism, because you do want open space, but you also want it structured, or closed, I should say. So I have the tape on the floor... that's defining a space. While you need all that open space, each child needs to know their boundaries in that open space. I use those spots...I put them around the room, so each child has their own space. The middle area is where the content is presented to them. And they're allowed to move on the carpet, not on the tile: the tile is the place where we play the instruments. Having certain areas of boundary has really helped. (interview one)

I could tell Karly had given the spatial arrangement of her room a lot of thought, which made me wonder if the arrangement played any role in facilitating collaboration. I remembered when I had visited her once before that she had all the students grouped tightly around her, at the piano, sitting inside the taped lines. She said that collaboration and proximity were related, in her opinion:

They have very quiet voices. They're going to get a much better sound if they're quiet. In a circle, singing, you can't really hear the person next to you. When they're close together... they can hear each other better, and I think it creates an intimacy. Like a chamber music feeling, or an ensemble feeling, and they're more willing to collaborate, as opposed to like, staring at each other, across the room, in 
a distant land! I think it really enhances collaboration...this [inside taped-off piano area] is where all the collaboration takes place. All of it. They never even mention anything when we're in that circle! It's only when we move over here that they say, "I have an idea!" but it's only when we get here. (interview one)

Karly's starting point. Karly had organized her thoughts on student music collaboration to a remarkable degree, thinking about how collaboration is affected by almost every element in the learning environment. I thought that our discussions in the CTSG would benefit from her critical and thoughtful analysis.

Karly spoke about collaboration as incontrovertibly tied to its musical context. While she alluded to the social and emotional needs and challenges of students working together, Karly verbalized collaboration as, at its core, a combination of musical ideas.

I also understood that for Karly, student creativity is firmly situated in, and reliant upon, collaboration. Rather than collaboration being just students listening to one another to maintain ensemble, in Karly's class collaboration might be students listening to one another to maintain ensemble in an arrangement of their own creation. This expanded view, of creativity and self-expression as a function of collaboration, was exciting to me.

I was fascinated with Karly's refined ideas on facilitating whole-group collaboration. A theme that often arises in my discussions with teachers in clinics and workshops is the idea that collaboration happens in small groups only. I thought Karly's expertise in the area of "whole-group collaboration" would be a valuable thing to pass on to other teachers.

Karly's differentiation between different facets of collaboration-variously, "meaningful," "not meaningful," "whole-group," “ownership," “personalization”indicate she is a thoughtful teacher looking for fine shades of distinctive detail within her 
classroom. I wondered how the discussions in the CTSG would influence her thinking, and what sort of effect her ideas would have on our group's understanding of collaboration. I hoped the CTSG would fulfill her need to talk about music teaching.

I noted Karly works in a district that places value on teacher communication and school improvement. However, Karly said she feels music in Edgeville lags other curricular areas in organization and improvement efforts. Her ideas, vocabulary, and knowledge of general educational and curricular reform movements, would well-equip her to spearhead efforts in moving Edgeville's music to a more progressive place. I wondered how her career would play out there, and if she would be able to provide musical leadership to the district.

\section{Part Two: CTSG Midpoint}

At the midpoint of the CTSG, Karly's eyes seemed opened to the various ways collaboration might look in different music classrooms. She was excited by the possibilities she had seen on Andrea and Marlene's videos and had already tried incorporating some of their ideas into her teaching. She had also taken note of what was particularly important to Andrea and Marlene in student collaboration, and started watching for those elements in her own teaching. Karly also valued the opportunity to examine her own teaching on video and hear the opinions of the other members; she went so far as to say the CTSG had made her a better teacher, already.

Karly's job: “I feel just completely rejuvenated.” In our first interview I learned that Karly was disappointed in both the scarcity and tone of music teacher meetings. In our second conversation, I understood better just how sad and angry she had been. Karly 
also told me that while she had felt frustrated by this lack of connection for three years, it was in part a self-imposed isolation:

I've been so bitter, just so bitter, so not wanting to call people [in Edgeville] on the phone and ask them about things. I think I purposely isolated myself because, honestly, people were so [growling noise] about certain things. I didn't want to share anything about what my kids did, and I didn't care what they did. And that's really sad. There are many people in this district, which I loved in the beginning when I got here, and just, because of how things have gone, I just don't care anymore. (interview two)

Karly said the CTSG had helped: "The fabulous part of this is that I feel just completely rejuvenated" (interview two). Part of that, for Karly, is that the CTSG is not about personal ego: "It takes away the endless, 'Oh this is my program, that is your program' talk. This is about the kids, you know. How can we really help them become better musicians and better citizens?"

I was surprised to hear Karly talk about the capacity of a CTSG, using a protocol, to help the most contentious group come together :

I'm thinking of certain people, in my everyday dealings, that I just stay away from you know? And thinking, "Wow, we could find common ground with a group like this [the CTSG], and I could actually learn something from these people, within a guided setting. (interview two)

\section{Collaboration with other teachers: "You can be honest and start getting to the}

nitty-gritty." In our first conversation, Karly told me that she was frustrated by the lack of specificity in Edgeville's music teacher interactions, saying they never got to the nittygritty of tough teaching situations. I asked her how the CTSG compared.

She went on to say, "The nitty gritty is the whole point of this group. I think we're able to get to the nitty gritty as far as talking about collaboration, and also talking about music. I wasn't sure how that would work" (interview two). She explained that, although 
we could learn to be even more specific about teaching, we had come a long way toward that goal:

We're three, well, four different teachers teaching completely differently. That's the amazing thing. We have totally different teaching styles and we're teaching different content and, I mean, everything's different. But I think we've been able to find common ground and common language to get through that. (interview two).

In our first interview she had railed against what she saw as our profession's inability to come to any sort of unified goals. She said Edgeville conversations were meaningless as the teachers and classrooms were too dissimilar: like comparing apples to grapes to candy. In contrast, Karly said CTSG teachers' different perspectives had allowed them to see and highlight moments in her own videos that she would never have thought to look for, and that she was able to give the members new things to think about: "That was getting nitty-gritty, and we found really great common ground to get there I thought," she said.

You see that things are possible no matter where you are. Like, Marlene's kids, she is in the worst possible situation and those kids are singing beautifully. Kids in Pleasantford, they're not just robots. They're creating music. The neat thing is that anything's possible in those three different classrooms. We might need to approach it differently and we might need to teach certain skills. So anything musically is possible. And even with collaboration anything can happen across three completely different classrooms. And that boggles my mind! That's fantastic! (interview two)

Karly also had an opinion on the progress our group had made in getting to that common ground, a process that to her has involved a necessary amount of time and establishing comfort with one another:

The first [meeting] is okay, and the second time starts to get better, and the third time you become really collegial with each other. You really learn how to interact. It's like having friends. You pick up on nuances about people, and you know what they're thinking and things like that. And that's when you can really 
be honest and start getting to the nitty gritty, when you feel really comfortable. I love the group. I think it's the best feeling going to that group. (interview two)

Student collaboration: "It looks totally different in Marlene's classroom and Andreas's classroom than it does in mine." In our first conversation, Karly was detailed yet direct when she talked about her definition of collaboration. She called it "a meaningful combination of ideas." In this second conversation, I asked Karly to recall CTSG discussions we had in the last section of the protocol, where we consider "aspects of collaboration in the music class." I wondered if she had any new thoughts on collaboration. Her answer indicates an expanded view:

I would still stick with a combination of meaningful ideas I think, but those ideas I guess is what I would change. Because the idea doesn't necessarily need to be musical...they're turning towards each other and they're willing to participate together, they're blending their minds together... Idea could be anything from, "We're combining instrumentation" or, "We're combining people." (interview two)

I asked Karly to explain more about how a meaningful combination could be a defined as a combination of people.

I had Brandon in here today, and he said, "I really want to work with Colleen because we've been coming up with this tune and she really knows me," he said. She knows him better than I know him musically, like, they get each other. And so they look for each other, and they look for each others' strengths. So that word idea, I guess, it doesn't need to necessarily be musical. It could mean so much more than music. (interview two)

I was surprised by this development. Karly was so definite in our first interview that collaboration was a combination of musical ideas. I asked her, "It sounds like you've expanded your definition a little bit. Was that based on seeing other types of collaboration, in the videos?"

She said: 
Yeah. But also our talk. I mean, I just think of it sometimes so black and white: "It's got to be musical. It's got to be musical." And [Marlene and Andrea] were being musical when they were teaching, but it wasn't their musical content that I was necessarily listening for. I was watching for, "how are the students interacting with each other?" And that's where the collaboration comes in, but it looks totally different in Marlene's classroom and Andreas's classroom than it does in mine. (interview two)

Professional development: "I wake up at 3 am thinking about it." I asked if the

group met Karly's need for thoughtful talk about the improvement of music teaching. She said, "I wish I had it [CTSG] every day:"

It's so amazing to be around energetic teachers, who really understand that you want to do better and you want to be the best teacher you can be. And they're not critical, they're constructive and they're affirming. It's just a really happy way to spend your time. And it also makes you a better teacher. (interview two)

I wondered how four meetings could make anyone "a better teacher." Karly said one example was that she had experimented with changing the structure of her lessons, in response to what she had seen Andrea do on video at the April 24 meeting. Andrea was praised for her scaffolding abilities in that meeting and the direct way she helped the students advance. There was a sequence in which Andrea moved the students step-bystep through a process, adding levels of difficulty to the task; Karly labeled that the "intense" part of the lesson:

I asked Andrea, "When in the lesson did you do that intense step?" And she said that was toward the beginning. I thought, "Maybe I'm wasting too much time with frilly stuff and getting them warmed up." So now, having that intense ten minutes at the beginning where they know I'm really down to business ...it's a nice feeling. (interview two)

I thought the above comment indicated Karly had expanded her views of what constitutes a collaborative class. The "intense" portion of Andrea's lesson is what I had initially thought was too directive, the first time I saw it on video. It was the beginning of the lesson and she was standing in front of the class, starting them, 
correcting them, and directing their process. Andrea's demeanor started the students off with a strong idea about where they were going to be heading as a class. When I took my eyes off Andrea and watched the students' actions, I decided they were just as drawn in to getting the chant, percussion, and movements in sync as Andrea was, and shared her goals in a collaborative way. When Karly said she had altered her lessons to incorporate a more teacher-led portion at the beginning of class I knew she had learned from Andrea. Karly said:

We get right to business. I teach them the new content right away. We might review a tune ... then we get right to the new content. They're totally focused, they're enjoying it, they're engaged in it, and then it's easy. As opposed to bringing them in, letting them move around, picking their own things to do, and then have them saying "I wanna do what I wanna do!" (interview two)

Karly also said that she had started to notice things in her own teaching that were important to other members of the CTSG. She cited Andrea's catchphrase: "we're working for that perfect ending" and said she was paying attention to the endings of her students' work.

Karly liked watching her class on video: "Seeing the actual effect of what happens when you teach, that's a really positive thing... seeing the video and generating this list of ideas, it's been a fantastic experience that I want to keep going" (interview two). To tell me how much she learned from the group, she contrasted the CTSG with other professional development.

[In the CTSG] you can't sit around in a circle and just talk about how awesome you are, without any concrete evidence. And what's the point of that anyway, really? You know it's about getting better at teaching. And I felt by seeing Marlene's video and Andrea's video I'm becoming a better teacher. (interview two) 
I wanted to find about more about Karly's becoming a better teacher, so I

continued to ask her about how and what she had learned. She said the CTSG teachers

help her identify and fix problems and also give her other options to try:

It's just been a great feeling to sit down with people, to talk to people, to learn from them, and to realize that the way you do it is not perfect. There are many other ways out there that are acceptable and fantastic. How can I model parts of their teaching? How can I aspire to become a better teacher, fill the holes in my own teaching? Even if I think something's going great, what's an alternative to that particular way of teaching that can spice things up a little bit? (interview two)

She said she considers the CTSG learning "constantly:"

I wake up at 3 am thinking about it. I talk things out. That's how I learn. This talk has inspired me so much to keep going. I think about [the CTSG] every day, it's constantly on my mind. When I'm teaching, it's "how can I make this better? Did we talk about this?" 'Cause that's my job, to make things better, and you guys help me out with that. The best teacher doesn't have all the answers, and the best teacher is the best teacher because they've asked a million other people how to do things. (interview two)

Karly said the discussion of her own video, emerging out of the protocol, was

illuminating: "I would never, never, never, have come up with even three-quarters of the things that everyone has picked up. I mean it's truly incredible" (interview two).

She said the first time she presented, she felt nervous. But the second time:

I was loving sitting back, watching it, enjoying it with you guys, and picking out new stuff for myself to notice. It's really exciting, 'cause you're like, "What are they gonna say? What are they gonna pick up?" And you're intrigued to see! (interview two)

Karly added she was glad the videos were an unvarnished look at elementary music: "if you show the perfect stuff all the time, you're not helping yourself, you're not helping other people either" (interview two). As an example, she said that one of our best conversations in the CTSG was when she showed a video of students who were having difficulty collaborating: they seemed uncomfortable and were not blending their voices or 
looking at each other. She said, "When we talked about why they seemed uncomfortable, I learned the most out of that moment, as opposed to moments that were beautiful and perfect" (interview two).

Karly talked about how fun it was at our meetings to go back and re-watch a portion of the video to verify something a group member had noticed. Karly remembered the time when she thought a little girl was giving her a disrespectful stare, but on the second viewing, we all thought she was really just thinking hard and probably audiating the tune. Karly said, "I mean that is the nitty-gritty! When everyone watches one child or something like that. You don't get to do that as a teacher" (interview two).

I asked Karly if she felt the protocol was limiting. She said the protocol is open enough, but she credits our meetings' productivity to the CSTG teachers' astute observations and questioning: "I think we could possibly limit ourselves if we don't ask the right questions. But I think I we've asked many great questions, about process, about content, looking for specifics" (interview two).

Karly likes the collaborative consultancy protocol that calls for the presenting teacher to show video without first setting up a context or situation for the viewers. She said that if we watched the video to solve a specific problem for the teacher, "we would be looking specifically only for those things and I think we would miss a lot of stuff" (interview two). However, she was clear that watching the video with the mindset of "collaboration" was what prevented the discussion from becoming a free-for-all. "You know, we are looking for something specific within a general setting. So that kind of binds us together. We're the 'collaboration people' you know [laughs]" (interview two). 
Karly's changing views. At the midpoint of the CTSG, Karly's had a larger view of the ways collaboration happens in different music classrooms. She took Marlene and Andrea's ideas and tried them in her own classroom, and even started examining her own teaching for things that Marlene and Andrea found especially important.

The most notable change I noticed in Karly at the midpoint was her enthusiasm and gratitude for the significant conversations about teaching we were able to have in the CTSG. Karly emailed me on April 5, one day after our first meeting, saying she was inspired and energized to teach. She said, based on something we had talked about at our meeting, she had told a class that they all had an important part to play:

It created this amazing energy in the classroom, and seemed to help make the children aware of how much they need to depend on each other to create a beautiful piece of music. I noticed students encouraging each other more than I've seen before and saw a bit more pride on the faces of...the "invisibles."(Karly, personal email, 04/05/08)

Karly spoke positively about the character of our discussions, and credited the protocol for enabling meaningful discussion. Karly had been wary of the pitfalls in talking to teachers who do not see music education her way. She depicted her past interactions with teachers with growling sounds to illustrate their acrimony; the CTSG , in contrast, she called "rejuvenating" and "fantastic."

\section{Part Three: At the End of the CTSG}

Toward the end of the CTSG, I sensed Karly had absorbed some elements of the CTSG members' practice within her own. More than any of us, she made explicit what she had learned from the others. The biggest changes I heard from her in her views of collaboration involved her defining collaboration in an expanded way: in terms of 
sequencing, community, direct instruction, and assessment. She also was able to verbalize tangible ways in which the CTSG met her need for teacher talk and learning.

Expanded views of collaboration. Karly incorporated a number of strategic sequencing ideas to enhance her student's musical collaboration. When she presented her video on May 29, 2008, much of our discussion revolved around the steps through which Karly took her students, and if the outcome would have been different had she placed them in a different order. This sort of minute discussion about sequencing and order made me think that Karly was giving more thought to how she could effectively facilitate collaboration.

Karly said, "I am fascinated with the whole idea of when to have them turn to each other and improvise, the whole taxonomy idea. Should there be an order? And I have an answer now!" (Karly, CTSG wrap-up meeting) She said that watching and talking about the process on video made her notice one or two gaps in her sequence: gaps that if filled with intermediary steps would have helped her students be even more successful.

Karly said all her instructions were geared toward giving students experiences to successfully improvise over a slightly different chord progression than normal, and how collaboration and working together could support that. She credited Andrea for making her think about structuring the steps for the students, instead of waiting to see if they happened on their own. She said, "I took them through more sequential steps than I usually do. Thank you, Andrea, can you tell you're rubbing off on me?” (Karly, CTSG meeting six). 
In our last CTSG meeting, Karly mentioned something she learned from Marlene.

She said:

I have been thinking all week, Marlene, about how when the kids are so quiet, sitting there... how it looks so good for an administrator, but they're not learning a lot. It just blows me away that we try to do that. Seeing your video completely gave me a wake-up call on that.

Karly also verbalized a new definition of collaboration, going beyond her initial view of collaboration as meaningful combination of musical ideas. On June 11, she explained how she was currently thinking about collaboration:

"[W]orking together, in the sense of community. It's not just about learning as an individual. It's working as a team, helping one another learn, performing with others. That's kind of what I assumed it would be, but it's kind of neat to remember that it was the same, across all of the videos and across all of our thinking. (Karly, CTSG wrap-up meeting)

Karly had come to see attention to the emotional and social aspects of collaboration as almost equal to the musical part. She was pleased with the list of ideas I collected in the summary handout dealing with how to create a caring classroom in which kids would want to collaborate. She said:

It would be really neat to share this emotional and social collaboration list with regular classroom teachers. I bet a lot of these are their best practices, too. The musical stuff is the content, specific things that can really help our profession. But if you don't have that emotional-social part, you're not really a teacher. (Karly, CTSG wrap-up meeting)

In the June 11 meeting, Karly talked about how collaboration is not a panacea for every problem in music education, and how she is frustrated by inconsistencies between teachers in what students should able to do. She is also frustrated by balancing content knowledge with her desire to provide collaboration space. She said:

The hardest part of this for me is that as music educators, we are just so far behind. So when these kids come in, and can't sing any song, not one, you feel like you have to be 'on' more. They need a model, and who else will be one for 
them? For me it's hard to say, 'okay let's do a group activity and create' when they haven't had enough of that model. (Karly, CTSG wrap-up meeting).

I challenged Karly: "Don't you think that being that model, and teaching them a lot of music, is not necessarily being un-collaborative?" Karly's answer indicated another expanded view of collaboration, one in which even teacher modeling could be imbued with a collaborative quality:

I suppose not. Non-collaboration would be anything that does not involve interaction. I've heard that word in here a lot. If they kids are interacting with the teacher, and conversing, and learning, with her, they're collaborating" (Karly, CTSG wrap-up meeting).

I asked her to describe "conversing and learning with the teacher." She said, "That's a perfect example of 'we're here, we're going to go through a process and we're going to get to a better product, and it's not because I'm drilling you" (Karly, CTSG wrap-up meeting).

The above exchange is an example of how Karly expanded her definition of collaboration into an interaction that could happen even when the teacher was in a direct instruction mode. In our first conversation, Karly made a clear distinction between the "presenting" and "collaboration" phases of her teaching. She had described direct teaching as something that had to be gotten through before the more fulfilling collaborative arranging projects could happen.

Now, she was saying that even during teacher modeling — teaching songs and bass lines for example — she could be aware of a balance between student and teacher input. She said, 'I'm going to listen and take it in when they suggest something. I'm not going to just say, okay we're moving on. I'm being aware of children trying to interact with each other" (Karly, CTSG wrap-up meeting). This sense of sharing with students and 
awareness of collaboration even during direct instruction was, to me, embodied in Karly's first video (I describe it in Chapter Six as Karly's ability to "provide, guide, then fade away") but this was the first time I had heard Karly verbalize it.

\section{Summary of Karly's Story}

Karly was the most enthusiastic CTSG member in verbalizing how the group supported her need for frank discussion, evaluation, and change of teaching practice. Karly was also the most vehement in her calls to organize, align curriculum, and create transparency within music education. She wants teachers to be honest and specific about what is being accomplished. My overall sense of Karly is that she is an extremely talented, dedicated teacher, who is disappointed in the lack of reform she has been able to carry out; she sees little potential for support from much of the profession at large for her calls for change.

Of the three teachers, however, I view Karly as the most content of the participants with how the CTSG affected her own teaching life:

That's one thing about this group. You can't argue with video. I've sat in meetings and talked till we're blue in the face about what kids can or can't do. But you can't argue with the video of a process that leads to a great product. You can try. But you can't really. And that has been the best part about this group. There's been more than one way to skin a cat, big time! And I saw proof of it. It's just been a really wonderful way to approach learning and it made me want to become a better teacher. I saw that it worked. (Karly, CTSG wrap-up meeting)

I also find Karly's views of collaboration more expansive. She began in the CTSG with a sophisticated and refined view of collaboration and right away was able to show, in the first video at the first meeting, an exemplar of a collaborative classroom. Her skills at facilitating a whole-class collaborative arrangement were remarkable. However as the CTSG progressed, I observed Karly pulling from the group even more detailed strategies 
for improving collaboration in her classroom. She began talking about intentional sequencing, promoting community, and awareness of student input even during direct instruction. The quality and depth of her reflection on these changes makes me believe that Karly will continue her journey toward creating an extraordinarily collaborative classroom.

\section{Conclusion}

In examining the participant stories separately and collectively in relation to research question two- How has the focus on collaboration in the CTSG changed the teaching practice of these teachers?-I find evidence throughout that Marlene, Andrea and Karly changed elements of their teaching in subtle or dramatic, yet highly individual ways. Marlene bucked the trend in her district toward quiet, seated classrooms in favor of a livelier collaborative project, and felt good enough about the outcome to try it again and videotape it. Andrea identified and named important elements of her teaching, and continued to reflectively consider their use in her classroom. She also provided more ways for students to collaborate and become independent. Karly continued to expand her horizons of collaborative possibilities, and made others' ideas her own as she incorporated them into her unique teaching practice. Because change in teaching practice is so personal, and so elusive to document, I have chosen to leave research question two answered only in the reconstruction here of each participant's story. No new truths will be discovered, or other findings illuminated, by trying to draw artificial connections between, and compare, the changes in each participant's practice.

However, in examining the three stories individually and collectively in relation to research question one- How do the participants describe their experiences in the 
CTSG? - I identified two major themes in common that will be elaborated upon in Chapter Five in terms of how they connect the CTSG experiences of Marlene, Andrea, and Karly.

First, the CTSG gave the teachers an opportunity to examine their own teaching practice in an organized way: an erstwhile infrequent happening in their hectic, often fragmented teaching lives. The thoughtful consideration of teaching practice was richer because of the valuable input and sharing by each member. Each of the teachers said the process of examination and talking contributed to their learning. The precise elements of the CTSG structure and format that enabled or disabled productive analysis, sharing, and resultant learning are detailed in the next chapter.

Second, each of the CTSG teachers is cut off from others in some critical way. Marlene, Andrea and Karly each spoke insightfully about people with whom they wish they could establish or strengthen vital communication: classroom teachers, music teachers, mentors, administrators, even students. In Chapter Five, I delineate the elements of the CTSG that partially combated the teachers' feelings that they were doing solitary, little-understood work. I also discuss the factors that prevented it from being an adequate substitute for conversations of consequence within a school or district. 


\section{Chapter 5}

\section{THE CTSG}

Guba and Lincoln (2005) describe the researcher in social constructivist inquiry as "a facilitator of multivoice reconstruction" (p. 196). In Chapter Four, I presented a reconstruction of each participant's voice: the findings in terms of each participant's story. Here, I combine themes from each participant into a cross-analysis of the three teachers' experiences, balancing between themes in common and individual perspectives I connect the voices of the participants with my own interpretations and conclusions for a collective reconstruction of the CTSG experience. In this analysis, I have organized the findings according to two broad categories that emerged: (a) CTSG as professional development that supports teacher learning and change in practice; and (b) CTSG as professional development that combats isolation.

Guba and Lincoln (2005) stipulate that social constructivist research rely on a discussion of "individual or collective reconstructions coalescing around consensus" ( $p$. 194). Patton (2002) likewise describes a search for consensus on a subjective truth within a community of learners. He paraphrases Niemeyer (1993) to say truth is "a matter of consensus among informed and sophisticated constructors, not of correspondence with an objective reality" (Patton, 2002, p. 96).

As I examined the data for common themes and consensus, it became clear to me that consensus arrived at in the CTSG could not exist in a vacuum. We are not an isolated 
community of learners, but rather one operating within a larger whole of the teaching profession. I decided in this chapter to broaden the sense of consensus by linking to this larger community. Thus, within the discussion of each finding, I relate it to previous literature. Information from past research provides a basis for my interpretation of these findings, and helps connect new information from this study with previously studied phenomena.

CTSG: Professional Development That Supports Teacher Learning and Change in Practice

In this section, six elements of the CTSG are discussed that positively or negatively affected the way the teachers and I saw it as a professional development experience that supports teacher learning and change in practice. These six elements are (a) the collectively generated knowledge in the CTSG; (b) the lack of development of teachers' musical content knowledge; (c) the discussion of students' musical content knowledge; (d) the necessity of video; (e) the importance of the protocol in effecting change in teachers' analysis of practice; and (f) defining and observing student work, versus teacher work. To frame the discussion, I begin by recounting the CTSG members' previous experiences with professional development, and how their experiences mirror recent depictions of professional development in the literature.

CTSG participants' past history of professional development. Professional development is defined by Feiman-Nemser (2001) as opportunities with the capacity to transform "teachers' knowledge, understandings, skills, and commitments in what they know and what they are able to do" (p. 1038). The CTSG participants did not describe their prior professional development experiences as transformative. In fact, they 
discussed their professional development experiences as mandatory, haphazard, and sometimes ill-suited to the improvement of teaching practice.

In our first interviews, I gave each teacher the same broad prompt: “Tell me about your experiences with professional development." Their responses depict professional development much as Conway, Albert, Hibbard, and Hourigan assessed it (2005a): "token days or hours of sharing good ideas" lacking "meaningful experiences where the voice of the teacher and the effects on students are being discussed and felt" (p. 8).

The CTSG teachers described professional development as irrelevant, a required event to be arbitrarily logged, and a district's clumsy attempt to serve too many teachers, too broadly.

\section{Karly said:}

Well, professional development in a school setting has been, basically, you come in during a professional development day and you sit down and you fall asleep and the reason for that is, because usually it has nothing to do with music. (interview one)

Andrea said:

We're required to do, I think it's six hours of professional development for our district. Meaning you have to be somewhere for six hours: clock in, clock out. And then once you're done with the 6 hours, you're done with your professional development. (interview one)

Marlene said:

The problem is that most of the professional development that they offer is not meant for music teachers. It is computers, a lot of computer training, but never a p.d. for music teachers. They even had aerobics. I remember going to aerobics! All kinds of things! (interview one)

Friedrichs (2001), in a survey of music teachers' professional development in California found that teachers listed as some of the most ineffective professional development that which was created for non-music teachers and which did not address 
music education. The CTSG participants wanted professional development relevant to music teachers. Marlene, Andrea, and Karly all talked about the frustration that arises when they have to go to non-musical training sessions: "You're just sitting there going, 'this doesn't apply to me"” (Andrea, interview one).

Although each CTSG member described trying to apply the non-musical professional development topics to her own classroom to some extent, something was definitely lost in translation. Hammel (2007), in a review of professional development literature, found music teachers were regularly asked to implement non-musical, schoolor district-wide initiatives. Andrea gave an example of her only partially successful effort to apply material from a non-music professional development course:

There were different strategies that they gave us that I thought, this doesn't make sense as explained in science terms, but I could do it when I'm teaching [music]. Those things were transferable, but some things are, some things aren't. We teach a different...I don't know what the word is... style. (interview one)

Bauer (2007) states the music education profession assumes certain forms of musical professional development, such as music conferences and workshops, will be effective. However, there is a lack of research bearing out their efficacy. Bauer's assertion was confirmed by the CTSG members, who said even the music-specific professional development experiences they had were not necessarily context-appropriate. Each participant said the music-oriented professional development offered by local and state organizations was not always applicable, musical, or pertinent.

Marlene wanted workshops that would give her material for immediate use: "things that you can use in the classroom. Something that I can say, 'okay, tomorrow I'm going to try that"' (Marlene, interview one). Instead she was disappointed in the way workshops offered by her district often required purchased materials or elaborate set-ups. 
She also found a lack of follow-through: promised handouts detailing the content of the sessions never materialized, and subsequent follow-up sessions were canceled or never scheduled at all.

Karly felt the "cute ideas" (interview one) from workshops are not as important as developing musical content knowledge: "Once you have the musicianship in place, then you can start having conversations... but those conversations, I really believe, can't happen unless everyone has a basis of musicality" (interview one).

Andrea said music professional development was not sufficiently targeted toward specific grades and learning environments, nor did it honor the ideas and expertise of attendees. She advocated for "more sharing" (interview one), as she wants to know what teachers are actually doing: "Let's talk about what we are doing in the classroom. What are we studying right now? What are our focuses?" (interview one). For her this talk would be more valuable and pertinent than advice from outside experts.

In fact, each CTSG member mentioned that she would like to share her own ideas with others and see how they would work in other settings. There seemed a need for CTSG teachers to know how what they do relates to what other music teachers do. Karly wanted to ask the question, "What does this look like in your building?" She envisioned a scenario where teachers would be fully aware of what students in other buildings were accomplishing, musically. She felt this would lead teachers to "really push each other...challenge each other" (interview one) toward higher levels of student achievement.

The idea of a two-way street between idea-gathering and idea-sharing was important to all three participants. Feiman-Nemser (2001) refers to the "problematic 
view of learning in which teachers 'get' knowledge or skills from outside experts which they somehow 'apply' in their work" (p. 1041). Feiman-Nemser goes on to describe an empowering, teacher-driven style of professional development, which can "tap local expertise and the collective wisdom that thoughtful teachers can generate by working together" (p. 1042).

The learning in the CTSG experience appears to differ from the "problematic view" above, and from the participants' previous professional development. The CTSG seemed to meet the self-avowed need of these participants to have relevant professional development that instigated learning through collective generation of ideas. The next section describes how the learning in the CTSG utilized Feiman-Nemser's "local expertise" and was therefore more locally applicable as well.

Collectively generated knowledge in the CTSG. The learning in the CTSG was generated by the group. Quotes from the participants indicate the entwined nature of the way they learned: from each other, about each other, and about themselves. We analytically examined Marlene, Andrea, and Karly's classrooms. As a result, everyone seemed to learn more about their own students and their own teaching, as well as others' teaching.

Andrea said, "How much have we learned about ourselves just by watching our students? So much" (Andrea, interview two). She also credited the CTSG for assistance in "analyzing my teaching, and the results of my teaching" (Andrea, interview two): “How much stuff did you guys get out of my teaching that I didn’t even think about? Tons!” 
Karly said, after the fourth CTSG meeting, "We've asked many questions so far. I'd like to even go back and look at all the questions, and write them up, and share them with other teachers" (interview two).

Marlene said watching her class through others' eyes, at the meeting, and hearing what others had to say about it gave her new things to think about: "you guys make me see what's good in it [my video]" (Marlene, interview two).

Karly also said she loved to watch her own video and compare what she saw with what the CTSG members brought up for discussion:

That's been actually the best part, going in thinking, here's what I do. And then picking up things, and realizing, from what you all are saying, oh! maybe they [students] do have a sense of meter! That's a really positive thing. (Karly, interview two).

I asked Marlene to compare the CTSG to other professional development opportunities she had had. She said the ideas suggested at CTSG meetings are immediately applicable and reliable by virtue of being vetted by practicing teachers:

The suggestions that you get from the other teachers are more relevant to what we're doing in the classroom, 'cause they have the same, or probably they have the same, situations, and not only that, they've been music teachers so they know what else you can do" (Marlene, interview two).

Marlene also said the CTSG teachers had a knack for finding creative, surprising solutions that differ from what her colleagues might suggest.

Andrea said: "I know I want to be a better teacher, and I know I've learned so much from Karly, and Marlene, and from you" (Andrea, interview two). In fact, the list of ideas collectively generated by the CTSG was a point of pride. In the May 29, 2008 meeting, I was planning the agenda for our final meeting on June 11, 2008. Everyone had 
presented video twice, so I asked if anyone would like to bring a third video. Andrea said she would rather see a compilation of everything we had talked about in our meetings:

You know all the ideas and suggestions and comments you've been writing down so far in our meetings? Could you collect all of those from the chart paper and the videos and make a handout we could go over and keep? I really want to remember all the ideas and suggestions we talked about. (Andrea, CTSG meeting six)

Her suggestion was greeted with enthusiasm, so I spent the next two weeks

compiling the questions and answers from our meetings into a five-page handout, categorizing the ideas and suggestions made.

At the last meeting, while we talked about the handout, the CTSG members made a number of references to helping other teachers learn what we now knew. I interpret this as a feeling of accomplishment: we collectively created a substantial body of information important enough to share with other teachers. The following quote from Karly illustrates the excitement the CTSG members felt about using their knowledge to help others:

If I were really new to collaboration, and wondering, "how do I know if I'm on the right track?" and I had [our] handout saying "oh you'll see these emerging behaviors," it would help a ton. (Karly, CTSG wrap-up meeting)

The lack of development of musical content knowledge for teachers. Professional development research highlights the importance of the interaction between content knowledge and pedagogy. Grossman, Wineburg, and Woolworth (2000) refer to difficulty in combining "two angles on teacher development—one focused explicitly on improvement of student learning, the other focused on teacher as student of subject matter" (p. 14). They found that intellectual development in the content area was necessary to promote teachers' views of themselves as not only experts but lifelong learners themselves, excited about the subject matter. 
The data from the CTSG meetings do not support claims that musical content knowledge was a factor in the learning of these teachers, or even a notable presence in our meetings. However, in analyzing the transcripts of my six semi-structured interviews with participants, I found evidence that all three participants might have welcomed more musical experiences at our meetings.

Karly asked, "If I can't hear Dorian, how am I going to expose my kids to Dorian? Honestly, if I can't improvise, how am I going to hold them accountable?" (interview one). She went on to talk about her view of strengthening content knowledge in becoming a better teacher: "Building our own musicianship first is a key. So it's almost like a top-down model" (interview one).

Marlene talked about Ridgeland's drumming sessions in which the learning curve was too steep for a one-time workshop. She said she didn't remember the rhythmic patterns, and that the notation was never sent to the teachers following the workshop. She tried to enroll in the same workshop again, to solidify her skills, but that attempt failed: "they had it [scheduled] again, and I wanted to do it again! And then when I got there, they didn't show up" (interview one).

Andrea explained that the music-making portion of the Greater Ridgeland Orff Schulwerk Association workshops was positive, as it helped her own musical growth and let her experience music as a learner again: "You get to try songs, actually play the parts so you really experience it...If you sing it, you're like, 'Oh! this is a cool tune and rhythm!' but if you just look at it, you think 'that's too hard!'” (Andrea, interview one).

I wonder if the CTSG would have been more effective with the inclusion of a focus on musical content knowledge. How might this musical content have been 
incorporated? As a facilitator and organizer of the meetings, did I fail the members in not providing an avenue to explore the really musical parts of teaching music? Junda (1994) found the balance between pedagogy, musical sight-reading, and increased song repertoire was one factor contributing to the positive outcome of a year-long music teacher professional development course. Should we have sung more songs in our meeting, improvised, or played our instruments together? For a group meeting just seven times, it would have been difficult to fit in other musical activities. The time in our group was well-spent investigating aspects of collaboration, but would the neglect of musical skill building have become more obvious if the group had stayed together longer? There are no data supporting answers to these questions.

The discussion of musical content knowledge for students. As discussed in the previous section, I found we spent little time talking about what musical content knowledge is most important for teachers. There was also little precise talk about what student musical content knowledge is important. While we spent a large amount of time talking about ways to identify and facilitate student musical collaboration, the specific musical content taught through collaboration was not discussed. Karly taught arranging and improvisation, Andrea taught round-singing, body percussion, and movement, and Marlene taught singing, beginning recorder, and beginning music literacy.

I was surprised that the type or quality of musical content taught by our members was not talked about more, or challenged. However, I do not see a lack of challenging talk or disagreement as necessarily negative. Each teacher's approaches and goals for her students were different, and a wider view of the possibilities in elementary music teaching was one of the positive results talked about by the participants. 
For instance, in the videos, I noticed that each teacher had different ways to organize and sequence a musical activity. In our second conversation, I asked Andrea if she now had an opinion on the best way to sequence instruction. She said, "All three of us have it [ability to sequence]... the underlying thing that's coming out is how different people sequence, but it all works" (Andrea, interview two). Arguments about what sort of musical content was sequenced by each teacher would not have helped us get to this sort of expanded view any faster, or perhaps at all.

Karly said she was aware of our lack of discussion about each teacher's musical choices, and chalked it up to a view that we trusted one another as professionals. She said that before participating in the CTSG, she saw good, musical teaching as a matter of "black and white" but the CTSG had expanded her horizon of what is acceptable and beneficial musical content:

[Marlene and Andrea] were being musical when they were teaching, but it wasn't their musical content that I was necessarily listening for... we're all good music teachers, and you trust that. The content's going to be there. (Karly, interview two)

I feel that Karly's students, extraordinarily advanced in their ability to improvise meaningfully with awareness of harmonic structures, may have intimidated us from some conversation about Karly's goals. It was hard to question her approach when her videos portrayed such musically facile students.

Marlene said her students could not do what Karly's did, and indeed, Marlene's videos depicted students who were beautiful, tuneful singers, but who did not appear to be musically independent. We never talked about how Marlene's students might move toward internalizing and improvising over bass lines, like Karly's do, and it was never intimated that they should. Instead we talked to Marlene about how to do what she 
already does with students more collaboratively, and how to empower more students to use their energy toward positive, class-wide goals. Her musical goals were never questioned.

Another example of a lack of outward disagreement about specific musical content followed Andrea's video presentation at the April 24, 2008 CTSG meeting. Andrea's students were advanced in their ability to create and maintain a musical ensemble. However, Karly's comments obliquely alluded to the idea that Andrea should have her students improvising, reacting to others, and engaging in a more musical conversation. She seemed to be viewing Andrea's video in light of her own definition of collaboration — a collection of meaningful musical ideas, put together in musical conversation - and subtly questioned how Andrea's students might go further in this regard:

That activity seemed so familiar to them, so I'm wondering if you could bring them that extra step musically and have them improvise a different rhythm pattern ...listening to each other and engaging with each other and conversing with each other and going to that next step of collaboration... if someone says "bah-bahbah-bah-bah-bup-bup-bah" [scat-sings an antecedent phrase] I have to process that in my brain and then say, "bah-bah-bah-bah-bah-bup-bup-bah" "[scat-sings a consequent phrase] back to them.... what would that look like? Would that strengthen their performance of future tunes if they hear what it's not in their head when they're performing it? (Karly, CTSG meeting two)

In this long statement, Karly was asking us if Andrea's students can really hear question-and-answer phrases in music, and furthermore, can they formulate and answer them, independently? This is a skill valued highly by Karly, and I believe part of Karly's black-and-white view of music education: either students are taught to hear chords, changes, and improvise, or they merely imitate the teacher who does. For Karly, and 
many music teachers, the former promotes musicality and independence; the latter does not.

Karly's idea was not explored further by the CTSG at this time. It is not clear if we habitually shied away from serious topics about organizing musical content for our students: topics about which we might disagree substantially. I searched the data for other examples of conflict and found little. This lack might be disappointing if viewed from the perspective of Achinstein (2002), who said this about conflict in teacher learning communities:

Conflict generates opportunities to strengthen communities, for in the conflict lies an occasion to examine difference of beliefs, solicit alternative voices, bridge across differences to find common ground, and seek opportunities for change and growth. (p. 449)

Achinstein, who studied interactions in whole-school communities, states that a "simplified and overly optimistic vision of collaborative reforms" permeates much research in teacher communities. She says that current research has left "the dilemma that is at the heart of community — how members really manage conflict amid unityunderexplored" (p. 422). Achinstein acknowledges, however, that what some educational researchers call moments of creativity and change through conflict, teachers call painful and frustrating. Repeated disagreements can result in teachers leaving communities, fragmentation of groups, and further isolation.

Achinstein studied entire school faculties operating in community; these groups through necessity communicate on a daily basis, if only superficially. The CTSG did not, nor did its members have much opportunity for daily communication with any other teachers. It is my sense that the CTSG members had enough fragmentation, argumentation, and isolation in their everyday teaching assignments. They may not have 
wanted to explore issues of great disagreement in our meetings. The CTSG was for us a place of support where understanding, communication, and realistic evidence of classroom practice were valued highly. Theoretical or methodological arguments did not seem to have a place in our protocol-oriented analysis of video.

Also, the topic of the CTSG may have lessened the need for conflict to break down barriers and then build bridges. Collaboration was a new subject of study for all of us so we approached with little preconceived ideas; most of our work was based on constructing definitions of collaboration in different teaching scenarios. Had the topic of the CTSG been "improvisation," or "the benefits of Orff-Schulwerk versus MLT versus Kodàly," the conversations might have forced more uncomfortable reconsideration of beliefs: perhaps productive, perhaps not.

The necessity of video. The analysis of classroom video contributed to the way the CTSG supported teacher learning. Little (2003) states, "accounts of classroom practice are generally opaque by comparison to lived or observed classroom practice" consisting of "condensed narratives" (p. 936). In contrast, our videos provided a transparent window into classroom practice. Having a tangible, visual referent provided a basis for substantial conversation about teaching realities.

Little's "condensed narratives" were related frequently in our meetings. Usually stories or anecdotes, these accounts were subject to the personal, possibly narrow, interpretations of the listeners. However, the video presentations widened our interpretations. They enhanced the presenting teacher's view of her own classroom and helped to correct viewer misconceptions. 
An episode that demonstrates the value of video in widening the group's view and eliminating preconceived notions happened at the CTSG meeting on May 1, 2008. Without the video she brought, Marlene might have told us about what it was like to push a cart of instruments into a crowded classroom, and try to teach singing and recorder to a group of children sitting at desks. In just hearing about this scenario, we might not have been able to make pertinent, relevant observations. We might have thought there was just no way learning could take place under those conditions, and advocated for Marlene to spend her valuable instructional time pushing desks out of the way. We might have dismissed the situation as impossible, and spent time strategizing with Marlene about how she could find a new job, or at least find a closet to make into a classroom.

Instead we watched her video and acknowledged the difficulty of the situation. Then as was our practice, we followed the protocol for describing the student behavior we saw on tape. Our viewing of the videotape led us to notice the number of students trying hard, watching each other, listening to one another, fingering along to practice, and singing beautifully. A large percentage of her students were behaving like musicians, despite the crowded, noisy circumstances. Marlene found our observations encouraging: "You guys make me see what's good in it... not just focusing on how difficult it is to work in the city schools" (interview two). Karly described the experience of watching for musicianship in Marlene's video: “Marlene's kids sing, I mean, holy moly, they sing. The kids sing really well. And it's like, she is in the worst possible situation and those kids are singing beautifully" (interview two).

The CTSG members thought our conversation would have deteriorated into anecdotal stories and tips without the video and the LASW protocols. The video elevated 
the conversation to high-level analyses of teaching and learning, of which the participants were proud.

That's one thing about this group. You can't argue with video. I've sat in meetings and talked till we're blue in the face about what kids can or can't do. But you can't argue with the video of a process that leads to a great product. You can try. But you can't really. And that has been the best part about this group. There's been more than one way to skin a cat, big time! And I saw proof of it. It's just been a really wonderful way to approach learning and it made me want to become a better teacher. (Karly, wrap-up meeting)

The participants found the assessment of their video illuminating, and eagerly anticipated hearing what the CTSG members would say about aspects of collaboration in the lesson. The comments made in the protocol enabled participants to analyze their own teaching and the results of their teaching. Because the discussion revolved around referential details related to the video, the analysis and suggestions were pertinent; as Karly put it, it was the difference between "seeing those videos and not just hearing about it" (interview two).

In Horn's (2005) case study analysis of informal communities of math teachers at two high schools, she found teachers' systems for talking about and categorizing students provides information on their beliefs about student learning. She explains, "Teachers' ways of rendering classroom events in conversation established norms of sharing or privacy, and when made more visible, provided windows into classroom practice" ( $\mathrm{p}$. 229).

Marlene struggled to reconcile her need to manage boisterous children with the sort of creative, musically collaborative lessons she wanted to teach. In my first interview with her she said that ideas from previous professional development courses often seemed like they wouldn't work for "this [Ridgeland] population" (interview one.) When 
I pressed her on what sort of ideas would work for Ridgeland students, she was vague, saying "things that are active" (interview one). She also referred to her job as "kind of hard" as and her students as "very tough kids" (CTSG meeting, meeting one).

These brief verbal categorizations of her job and her students gave me a sense of Marlene's beliefs; she has a hard job and tough students that need a kind of particular, yet undefined, learning environment that will work for them. As the sympathetic observer, I adopted these beliefs as my own about Marlene's situation.

After the CTSG May 1, 2008 meeting at which we watched and talked about her video, Marlene went back and tried some of our ideas. We had geared our suggestions toward Marlene's exact situation and students, a specificity we were only able to attain because we had seen her environment on videotape.

As Andrea put it, before seeing the video of Marlene's classroom, her ideas and impressions of teaching in Ridgeland were vague and fictionalized: "I told Marlene this: I could seriously never picture myself in her position... Like I had these images in my mind of like what it was like to teach in the city school district" (interview one). After seeing the video Andrea had a much deeper understanding of Marlene's exact situation. She was able to suggest harnessing the students' enthusiasm, energy, and competitiveness by having them work in groups to teach one another. At the May 9, 2008 meeting, Marlene reported that Andrea's suggestions, slightly adapted, worked. She was proud and happy.

Andrea's video presentation helped us understand the reality of her teaching circumstances in a way that her descriptions did not. In the first CTSG meeting, she described her students as compliant and obedient: "Pleasantford kids...they mostly just 
want to please you" (Andrea, CTSG meeting one). I was skeptical about this description, never having experienced anything like "Pleasantford kids" in my own teaching. I

prompted Andrea to imagine a student who might be outwardly cooperative but inwardly disengaged, saying, "I could envision some kids taking the path of least resistance. In their head they're not really into it, they're going to just mouth along” (Ann Marie, CTSG meeting one). Andrea explained that no, the most rebellious her students get is refusing to use their singing voice: "For me, it's the ones who don't have a singing voice and don't care that they don't get a singing voice, it's just like, 'whatever, I don't have it."'

This time, rather than adopting Andrea's beliefs about her students as I had done with Marlene, I remember thinking that we should all be so lucky to have chest-voice singing as our biggest teaching problem. I thought skeptically we would probably see a few bigger problems than that in Andrea's video.

However once again the reality of video expanded my preconceived ideas in a way that Andrea's condensed narrative about "Pleasantford kids" had not. All of us in the CTSG commented on how outstandingly cooperative they were.

Marlene said, “They [Andreas's students] would do everything that you asked them to do" (CTSG meeting two).

Karly added, "Most of them were really engaged most of the time. They were right with each other, they were listening, their ears were on and that's a really hard thing to have a class stay fully engaged the entire time."

I said the students were "amazingly attentive" collaborators. I saw students watching each other, leading, following, and in general, completely drawn into the whole 
activity. I was able to understand more about the sort of population Andrea worked with, and realized that at this school, blatant use of chest voice might indeed be a rebellion.

The participants also appreciated the opportunity to watch their own class on video and see different things than they had in the moment. Marlene said, "when I'm teaching, it's like I see chaos! It's much better to see it here, to see what really happened" (interview two). Andrea said watching her students gave her a way to understand her own teaching: "When you're watching the video, you're not watching yourself, you're watching to see what the students are getting out of it" (Andrea, interview two). Karly described it as, "Seeing the actual effect of what happens when you teach-that's a really positive thing" (interview two).

Borko (2004), in a review of professional development literature, states records of practice — lesson videos, student work samples, lesson plans — are powerful for teacher learning. Records help to “bring teachers' classrooms into the professional development setting. Such records of practice enable teachers to examine one another's instructional strategies and student learning, and to discuss ideas for improvement" (p. 7). Using video as a record of practice in the CTSG enabled such examination and discussion.

A final example of the profound influence of video lies in what I thought my reaction would be to the participants' teaching, and how that clashed with what I actually saw. Because Karly orients her teaching around Music Learning Theory, I had the notion that Karly's class would be more focused on improving and evaluating student achievement. I thought it would feature a great deal of repetition to improve skills in audiation and answering tonal patterns. Because Andrea orients her teaching around OrffSchulwerk, I had the notion that Andrea's class would be playfully creative and 
improvisatory. Because Marlene has taken advanced coursework in Orff-Schulwerk as well as Kodàly, I thought her classroom would be a combination of those methods.

None of these preconceived ideas turned out to be completely correct. In fact, some of my preconceived ideas were completely inaccurate. This made me realize how teachers' practice can be evaluated and judged based on shorthand verbal descriptions that are freely bandied about. Video can provide a more complete picture of a teacher's orientation and style.

The importance of the protocol as effecting change in teachers' analysis of practice. The protocol anchored the conversation and kept us dialoguing productively. Researchers in professional development have determined the importance of what teachers are able to bring forth or make visible for discussion, and how subsequent conversations may be constrained or encouraged. For example, a teacher's problem will necessarily be framed by how she depicts it. The way she talks about the problem may lead others down a certain path. What the teacher chooses to share is up to her; Horn (2005) states that teachers define what is sharable by "the rendering of practice in conversation" (p. 211).

The collaborative consultancy protocol partially eliminated the need for a verbal rendering of practice. A logical first step in looking at classroom video might be to have the presenting teacher set the context and explain what we were about to see. Rather, the protocol calls for a viewing of the video first, and for the non-presenting teachers to merely describe everything they noticed. By using the protocol, the CTSG sidestepped the potential problem of being limited by what teachers decide will be sharable. We 
praised the collaborative consultancy protocol for enabling forthright, objective classroom views.

Andrea said about the collaborative consultancy before her April 24, 2008 presentation of video: "I think it [the protocol] fosters more conversation. 'Cause you guys will see things that I won't see.” Karly agreed: “I was just amazed, too, how dead on you guys were [at the last meeting]. I mean, with a lot of the stuff that you were figuring out before I even had a chance to talk" (CTSG meeting two). Karly acknowledged how difficult it is to talk about teaching with others without prejudicing them with too much background information, and recommended instead to let the work stand on its own: "Let the work speak. Instead of saying, 'my kids can do this' it's much more powerful when the kids actually do it. So this [protocol] is really cool" (Karly, CTSG, meeting one).

The participants felt that had they predicated their video presentation with a specific problem, the other members would have been constrained to looking only for examples of, and causes for, that problem. Because the collaborative consultancy protocol requires the presenting teacher to show the video with no introduction, and to refrain from comment as the other group members describe it, the subjects available for discussion are wide-open. As Karly put it, if the presenting teacher asked the group to watch the video with an eye to solving a particular problem or analyzing a specific element of student behavior, "you would be looking specifically for those things and I think we would miss a lot of stuff" (interview two).

Andrea gave an example, from the May 22, 2008 CTSG meeting. In our second conversation, she reminded me of how fascinated we were by the rhythmic motions of a 
couple of students in the background of Karly's video. As a group, we were interested in their collaborative interactions: they seemed to be helping each other feel the beat during a time in class when Karly's attention was on solo improvisers in the front of the room:

Karly had the two kids in the background who were moving around to the beat when the other people were improvising. What if her problem was, "this one girl can't end on do." And we would have focused on that, figuring out why the girl in the front can't end on $d o$, and probably would have completely blown past the kids in the background who were really interacting with each other and feeling the beat, and that was the real collaboration. (Andrea, interview two)

In a study of six teacher communities called "Critical Friends Groups (CFG)," Curry (2008) addressed positive and negative facets of the use of protocols. She found protocols enabled groups to "engage in focused conversations about practice that ran counter to traditional occupational norms of teaching, like privacy, noninterference, conservatism, and congeniality" (p. 764). In Curry's research, participants favored the use of protocols, stating their usage "significantly enhanced the level of discourse and meaning constructed in CFG meetings" (p. 764).

However the protocol also limited the teachers' discussion. Curry found that teachers following a protocol often dropped lines of inquiry in order to go on with the next step. She contends this "weakened their capacity to deeply and collectively push on critical and commonly shared matters of practice" (Curry, 2008, p. 767)

I found no evidence that the protocol stopped our discussion on critical topics. None of the CTSG members said the protocol limited them. Andrea said: "if anything, [the protocol] broadens us just to talk about whatever we see" (interview two). Little (2003) defined "affordances" (p. 939): the ways in which the practices of the group either enable opportunities for wide-open discussion, narrow them, or shut them down altogether. Our group practice - using the collaborative consultancy protocol to organize 
a discussion of classroom video — afforded us the opportunity for far-ranging discussion of music teaching and learning. Nothing was limited, other than that we were trying to concentrate on aspects of student collaboration.

Defining and observing student work, versus teacher work. The classroom videos usually showed a wide-angle view incorporating teacher and students. However, transcripts of the meetings indicate less conversation about teacher behavior than about student actions and musicianship. This finding indicates the CTSG concentrated more on how students learn music, rather than on finding "better" ways to teach or sharing lesson plans. The opportunity to study students at work in a music classroom also fostered discussion of what student work in elementary music really is: process or product?

Our practice of studying students working in music class dates to our first meeting. Karly helped us establish important norms through the choice of content in her video. The "Looking at Student Work" protocols (Allen \& Blythe, 2004) call for the observation and study of student-generated paperwork and artifacts, not video. To apply the protocol to video documentation of students in a music class, we needed to decide for ourselves what would constitute student work for the purposes of our CTSG. Karly's video served to provide our initial definition of "student work" in an elementary music classroom. It showed children at work, singing, playing instruments, and engaging with songs through improvisation and bass line singing. The video culminated in an informal performance of a class-created arrangement.

We did not make the definition of student work explicit at any point throughout our meetings; in fact, we did not even try to define it until our very last meeting. The teachers were free to bring any video that portrayed students in music class, and the films 
varied. However, as the meetings continued, we talked consistently about the same elements that came up in our first discussion: student behaviors (musical and nonmusical), interactions among students, and student interaction with the teacher. It became a group understanding that for us, "student work" in music class meant "students working:" the process of students engaging with musical material. At our last meeting Karly summarized why, given our focus in collaboration in the CTSG, we needed to see the whole process:

The neat thing about this was that we were not looking for a specific product, we were looking for collaboration, so, bringing in the product would have been inappropriate for our purposes, completely inappropriate. (Karly, CTSG wrap-up meeting)

In our last meeting I asked the CTSG teachers to define "student work." They were clear that in music, student work is "whatever the students are doing to work to make music in class" (Andrea, CTSG wrap-up meeting). I asked if it is a performance, and Andrea said no, it is participation:

It's how are they participating? How are they singing? How much are they listening? That to me is work. They can't just sing to sing. They have to be working at singing: making sure they are matching other people, matching pitch, making sure they're coming in at the right time. That's constant work that they are doing. That's what we videotape and look at: their work. (Andrea, CTSG wrap-up meeting)

Marlene agreed that when she thought about student work, she was looking at students in the process of doing something, and not the result of that process:

When they are singing, that's their work. They have to come in at the right place, they have to stop together and listen to the other voices, or listen to the accompaniment...Y You have to listen, and think and be aware. (Marlene, CTSG wrap-up meeting)

I said that in the LASW guidebooks, student work is something that can be placed on the table and talked about, like a persuasive essay, or a piece of artwork. Karly 
explained why looking at a finished product like that would always raise the same questions for teachers; questions that we always had answered for us because our videotapes depicted classroom happenings:

It's the product that's within the process. You could technically record five kids singing the same thing, and we could analyze and compare those, but looking at the process of how they got there is more important. 'Cause one of the things that always came up in the LASW meetings I was in before was "what was your process? how did you get here?" and teachers would have to explain it. But we got to see the process AND we got the product. We got both. We got the student work, we heard the student work, in this music class, as it advanced. (Karly, CTSG wrap-up meeting)

The "Looking at Student Work" protocol also led us to more discussion about student work than teacher work. While I cannot say if the CTSG members watched the teacher or the students more on the video, I can relate an incident from the April 24, 2008 meeting which illustrates the difference. In watching Andrea's video, I changed my opinion of what was happening when I purposely switched my focus away from Andrea and toward watching the students at work. This resulted in my characterizing Andrea's classroom in a vastly changed way.

Andrea had videotaped a lesson in which the students learned a rhythmic chant and accompanying motions, transferred the chant text to body percussion, and then performed it using movement, in canon, and in variation. It seemed to be a textbook OrffSchulwerk lesson. I was frankly astounded by how compliant and eager the students were. I remember wondering if they were the world's most obedient class!

I formed an opinion in the first moment of the lesson. I saw Andrea leading the students through a chant, call-and-response style. She was standing in front of the class, starting them, correcting them, and directly shaping the process of transferring from chanting to body percussion. My knee-jerk reaction was that this is direct instruction, not 
the process of student-initiated discovery that I would expect to see in a collaborative lesson.

About two minutes later, my opinion started to change. I started noticing students were every bit as involved in getting the chant, percussion, and movements correct as Andrea was in teaching it to them. I thought, "This is more symmetrical than I saw at first." I noticed Andrea asking students for their ideas, and I decided that they were actually partners with her in this project. I saw students watching each other and working together happily.

I decided I needed to be open-minded. The power of watching student behaviors on video had given me a new look at collaboration, one in which I examined the students' actions for evidence of attempts to construct and maintain shared understandings. I had been fixated on Andrea, but I realized concentrating on the students at work would yield a more complete understanding of the phenomenon of collaboration.

Andrea seemed to share my realization:

I remember when you [Ann Marie] told me that we weren't going to be critiquing how we teach, that it was going to be talking about student collaboration, and I was like, "Uh, I don't know about that!" [laughs] You could probably tell I was kinda iffy on that. I thought, "how are three teachers not going to actually think about how Marlene teaches, or how Karly teaches, or how I teach?" But seriously, you were right. It has not come up in the sense of "What are you doing wrong?" or "What are you doing right?" It is more towards "what are the students doing?" (Andrea, interview two)

This finding does not mean we never talked about the teacher's ways of instigating or facilitating collaboration. We talked a great deal about how the teacher can organize collaborative experiences for her students, and came to important conclusions about the role of the teacher. The point is that some professional development experiences in the CTSG members' experience had been solely devoted to discussion of 
what the teacher does or can do better; the CTSG enabled a look at the role and work of both teacher and student.

\section{CTSG as Professional Development That Combats Isolation}

Each teacher in the CTSG had a different outlook on her ability to connect with colleagues, and a unique way of describing her role within the larger communities of her school and district. The particularly distinguishing features of each participant's interaction with colleagues are presented in detail as a part of each story in Chapter Four. However, themes related to the connection and support available within the CTSG, but absent from local district scenarios, are described in this section. First I will summarize recent research that depicts the isolation of many music teachers' work environments.

Unique challenges for isolated music teachers. Music teachers have cited isolation and a lack of access to meaningful discourse in music education as two longterm challenges to the quality of their teaching (Conway, 2003). This isolation compounds the inefficacy of the short in-service or seminar professional development model, as chances are music teachers will not able to confer or work with another teacher on whatever new ideas might have been presented.

Music educators' jobs, when compared with their colleagues' in other subjects, are faced with unusual "logistical and locational differences and difficulties" (Haack, 2003, p. 10). Music teachers are often the only one in the school, and may be itinerant. " $[\mathrm{N}] \mathrm{o}$ other career usually presents its aspirants with the challenges of working at two or three different levels, under two or three different principals, in differing schools and classroom settings, with two or three different faculties and staffs" (p. 10). 
Even within one school music teachers may not feel a strong association with colleagues. Scheib (2006) states music teachers are prone to isolation, because they have few colleagues with similar histories and experiences. He contends that many fine arts teachers come to teaching through their love of the subject matter, and suffer a loss of identity when no longer surrounded by others who are actively creating in the arts. Scheib concludes that fine arts teachers need support through socialization into their roleidentities, and suggests that collaborating with colleagues is a valid professional development activity.

For music educators, opportunities to come together and have ongoing conversations about music education are rare, but can be powerful. A teacher in Conway's (2003) examination of music teacher professional development said:

Professional development for me now is the same as it's always been - trusted advice of colleagues whom I admire. The most I tend to get out of any conference is the collaboration with colleagues... the conversation with colleagues is and always has been the most helpful professional development. (p. 155)

The CTSG members and isolation. The CTSG members cited a large degree of isolation within their schools and districts: a sense of not being "real" teachers, not fullfledged members of their school communities. Each participant had a story to tell about how classroom teachers saw them as "break time" providers (Andrea, interview one), intruders who are "too noisy" (Marlene, interview one), or "completely separate... 'special' area teachers" (Karly, interview one).

Connections within the music education community were no stronger. The participants had not formally observed other music classrooms since student teaching and felt cut off from what other music teachers and students really do. District-wide meetings of teachers did not serve these teachers' need for community as meetings were 
infrequent, irrelevant, or contentious. Participants stressed that meaningful talk with music teachers rarely happened outside the CTSG.

After the meetings were underway, the CTSG members spoke glowingly about the connection with the other teachers. Karly said, "I have been isolated for three and a half years... it is so amazing to be around energetic teachers" (interview two). Marlene said the meetings were "like therapy" and that she always felt better the next day (interview two). Andrea described the experience as "really, really fun" (interview two).

CTSG members felt inspired by and thankful for our meetings, and expressed a wish to meet more often. I received an email from Karly the day after our first meeting which opened, “I can't say enough how much I enjoyed Thursday's meeting. I was inspired by our conversations and energized to teach yesterday... a couple of points made which were particularly inspirational." After some discussion about what she had learned from the meeting, the email closed: "I just wanted to let you know how meaningful Thursday's meeting was for me and how much it affected my teaching even in the course of a few hours!” (Karly, personal e-mail correspondence, April 5, 2008).

I was stunned to get this e-mail. I had no idea that one meeting could make such a difference to one teacher. I did recall that Karly had told me in our first interview she was so desperate to talk music education, she would talk to herself. A few weeks after this email, in our second conversation, Karly told me that she was so jazzed up about the CTSG that she felt like talking to the wall, or to her husband in the middle of the night.

However, it is important to note that as enjoyable and beneficial as the CTSG was for all of us, the participants were clear that it was not an acceptable replacement for meaningful discussions within their own districts. At our last meeting, I directly asked 
them if the CTSG was a substitute for the kind of local teacher collaboration they wanted. There was a dead silence. Andrea finally said, "Well, it's beneficial but not as beneficial as if it was, say, a Pleasantford elementary music teachers group. It has been great to get perspectives from other teachers in other districts though" (Andrea, CTSG wrap-up meeting).

Marlene agreed with Andrea's assertion, saying that a CTSG of teachers from the same district would provide locally pertinent advice: "yeah, because if someone's been in your exact same situation, then they can tell you what to do in that situation" (Marlene, CTSG wrap-up meeting).

Andrea picked up on that statement and theorized to Marlene that it could be more helpful for her to get advice from other Ridgeland teachers, teachers more intimately acquainted with the unique challenges of that district: "It's hard for me coming from Pleasantford to say 'I really know what you should do in your classroom' because honestly there are some things where I don't know how to help you" (Andrea, CTSG wrap-up meeting).

However, Marlene pointed out that just teaching in the same district is not a guarantee that someone will be helpful:

Yeah, but I see the other music teacher [at school 100] teaching her class and she has the same problems, with the same really tough classes, and I don't know what to tell her either! And she can't help me that much. (Marlene, CTSG wrap-up meeting)

I interjected my opinion next: that to study music teaching and learning it was not necessary to be working in the same district, and perhaps it was better for us that we represented diverse situations. However, an intradistrict CTSG would undoubtedly reap 
benefits in terms of community and goodwill. I asked what else would be good about having a CTSG with members of their own district.

The CTSG teachers brought up the lack of curricular alignment they perceived within their districts, and how ineffective attempts toward unification had been. They thought the CTSG could provide a forum for realistic talk with other teachers about what is actually happening in the classroom, talk which might move beyond yet another rehashing of a curriculum document.

Karly said the lack of honest conversation about the elementary music curriculum was a “joke” in Edgeville: “There's a curriculum there but I don't follow it. And I get away with it. Like in math you would never get away with that" (Karly, CTSG wrap-up meeting). Andrea agreed: "In Pleasantford we have a curriculum but I guarantee no one follows it. Like I do my yearly planning, I structure my year around the curriculum... I don't think any other teachers in the district do that. They just kinda do their own thing" (Andrea, CTSG wrap-up meeting). Marlene said when she came to Ridgeland, she became aware of a tacit agreement that instructional design ideas from the central office were not mandatory for music teachers: "She [head of department] gave us these long lesson plans during orientation, and being a new person, I think I'm supposed to make these elaborated plans, and [mentors] said 'no, just forget about what she says"' (Marlene, CTSG wrap-up meeting).

Karly said, "See, that freedom kind of screws us over in a way." I asked her what she meant and she told us that different teachers teaching autonomously resulted in a disjointed experience for all:

I sympathize with the $3^{\text {rd }}$ grade teacher next year my kids are going to because she's not going to know what they can do-although I've called her a million 
times, so I can explain what they can do, and she's never returned my phone calls. They're going to go in with these skills and the teacher's going to say "What did you learn?" cause they're not going to exhibit the right skills... The communication's just really lacking, I can't give her the full curriculum that I do, because we don't meet, so she has no idea what they do, and then they go to the middle school, and then the high school, and then they graduate not being musically literate because no one knows what's going on. (Karly, CTSG wrap-up meeting)

This exchange indicates that while the CTSG members were personally inspired, rejuvenated, and educated by our meetings, the CTSG did not fulfill their need to be productive members of a district team. The CTSG teachers are frustrated by their districts' endless discussion about curriculum with little implementation. The lack of honesty about curriculum contributes to their sense that they are on their own, left to work toward their own goals, alone.

Research in teacher professional development highlights the importance of teacher support on the local level. Garet, Porter, Desimone, Birman, \& Yoon (2001) found, in a sample of 1,027 math and science teachers, effective professional development featured coherence between learning opportunities and teachers' actual classroom practice. Coherence is a direct link between professional development and teachers' daily experiences, with an effort to align outcomes with local, state, and national standards.

Penuel, Fishman, Yamaguchi, and Gallagher (2007) built upon and augmented Garet's (2001) construct of coherence and collective participation by stipulating that effective teacher learning occurs through "professional development in which teachers participate alongside colleagues from their school and district” (p. 929). Penuel, Riel, Frank, and Krause (in press) found professional development involving multiple 
participants from a school builds trust, relationships, and support for implementing new ideas.

The CTSG in one sense had a direct link with the participants' classrooms: we talked about individual students and particular schools. However there was a lack of alignment with district objectives, and no school-specific support in place for the CTSG participants. While the CTSG participants said the experience opened their eyes to new ways of looking for and enhancing student collaboration, I think being able to talk with their colleagues about these new ideas might better solidify their enactment in practice.

\section{Conclusion}

The CTSG was an effective professional development experience for these teachers. The CTSG is a structure that supported teacher learning through conversation and significant discussions. Our talks helped meet the teachers' need to belong to a profession, in a larger sense. The body of professional knowledge we codified in our meetings contributed to their sense of growth.

The CTSG also remedied feelings of isolation for these teachers. Meeting with colleagues energized the teachers and gave them new ideas appropriate for their context. However, the CTSG did not substitute for the teachers' desire to have on-going conversations with their school and district colleagues. The CTSG teachers were consistently frustrated in attempts at the school and district level to have any sort of conversation at all about teaching and learning, much less accomplish curricular alignment or reform.

These two themes - the CTSG as effective professional development and as remedy for isolation - are symbiotic. In reflecting on the close connection between these 
two findings, I can surmise that a completely fulfilling experience for teachers who want to learn and grow will contain elements related to both. Professional conversation, highquality, in-depth ways to analyze teaching and learning, and community are all necessary factors that combine and work together to strengthen and improve teacher development.

Chapter Five was a collective reconstruction of the CTSG experiences. I combined themes from each participant into a cross-analysis of the three teachers' experiences, balancing between themes in common and individual perspectives.

I begin Chapter Six still dwelling in a recounting of the CTSG's collective beliefs. However, rather than looking for themes in common as in Chapter Five, in Six I take a chronological, or evolutionary tack. I trace the emergence and development of our socially constructed definitions of collaboration. During the course of the CTSG meetings, our group's understanding of collaboration evolved as our definitions converged and diverged throughout. We negotiated a conceptual understanding of what student musical collaboration is: achieving an understanding held in common, marked by shared language, and reparations of divergences.

From my interpretive vantage point as researcher and group member, I then draw three principles of collaboration from our socially shared definitions. I reconstruct three vignettes from our meetings which illustrate our principles. The list of practical applications for collaboration in the classroom that accumulated throughout our work in the CTSG is found in Appendix E. 


\section{Chapter 6}

\section{COLLABORATION}

From the beginning, the CTSG members were conscious of two dimensions of this study: we were collaborating in a teacher study group to talk about collaboration in the classroom. Other than making this dual purpose clear, when beginning this project, I did not try to define student collaboration in elementary music for the CTSG members. Although I had opinions and thoughts garnered from past literature, my previous research (Stanley, 2007), and my experience teaching elementary music, I wanted to leave the concept of collaboration wide-open. My first interviews in March 2008 with Marlene, Andrea, and Karly centered around collaboration in a general sense; we did not establish specific criteria for identifying collaboration in elementary music.

Collaboration is a broad word with varied usage in educational literature. Music teachers may not have a clear picture of what it is and why it should have a place in elementary general music classrooms. The characteristics of collaboration overlap those of many other pedagogical schools of thought: constructivism, active learning, studentcentered learning, project-based learning, and communities of learners, just to name a few. The ambiguity of "collaboration", and the many images the term evokes in teachers' minds, might have made it an unwieldy topic of study for the CTSG. However, as the CTSG meetings unfolded and we discussed aspects of collaboration we saw on tape, we noticed many ways collaboration is made manifest in the classroom. We talked 
about its purpose, function, and defining characteristics. The complex character of musical collaboration emerged as a rich element of elementary school music: something we believed worthy of study and analysis. We eventually understood a great deal more about collaboration, and why it matters for elementary music. This understanding is what the teachers in the CTSG can share with others, in answer to research question three: What can these music teachers tell other music educators about collaboration?

Our definition of collaboration did not stay static. We talked about numerous types of collaboration, from musical to social to emotional. Despite the different ways we used the word, as the project proceeded, we were able to relatively quickly converge on shared understandings of collaboration. I credit that accomplishment to our thoughtful discussion of video evidence. We continually brought our discussion back to aspects of collaboration we could see: aspects that were visible on video and that we could talk about in terms of classroom events.

At the CTSG meetings, we did not refer to previous research to create our definitions. We analyzed the videos according to a protocol that called for us to identify and examine elements of student collaboration on film, not to determine if our views coincided with collaboration as previously delineated in the literature. As I paired my recreation and analysis of our discussion with a more theoretical, research-based component, insights about elementary music collaboration emerged that were not immediately apparent at our meetings. Therefore, the discussion of findings in this chapter is based on the collective wisdom of the CTSG, my interpretations of our understandings, and my knowledge of research on collaboration. As I found links between our definitions and past research, what is most interesting is how closely the two 
coincide despite the lack of preconceived ideas we began with as a group. This alignment with prior thinking and writing about collaboration provides further support for our definitions and principles.

\section{Structure of the Chapter}

I begin the chapter by tracing, chronologically and thematically, definitions of collaboration as they were socially constructed by the CTSG. Our group's understanding of collaboration was an evolutionary process; the definitions converged and diverged throughout our meetings.

In our discussions, we tended to sum up multiple aspects of collaboration into larger, unifying phrases. These broad statements about musical collaboration, such as “everyone stays musically involved" and "students take ownership," recurred throughout our talks. They became our tenets of the collaborative elementary school music classroom. From both elements - our definitions of collaboration and the recurrent broader phrases - I have distilled our three principles of collaboration:

1. Collaboration facilitates student self-expression and independence.

2. Students who are collaborating share goals. The teacher allows space for, or guides students in creating, productive student-student interactions.

3. A teacher collaborating with her students facilitates their movement toward a shared goal. Teacher provides necessary background skills, creates student buy-in for the goal, and then fades away to allow students to take ownership.

In the second section of this chapter I present our principles in conjunction with three vignettes. The vignettes, which relate to classroom videos presented at CTSG meetings by Marlene, Andrea, and Karly, exemplify our principles. In the vignettes I 
describe the events we saw on tape, and I recount our efforts to understand the collaboration enacted in each video. I unpack our principles of collaboration within each vignette, connecting our principles with past research as well as with what we learned about collaboration in the CTSG.

An additional body of knowledge constructed by the CTSG is a set of practical considerations for the application of collaboration in the classroom. The intense discussion in our meetings about what really happens in the elementary music classroom was reinforced by the realism of video evidence. Our discussion was not theoretical. We identified and described considerations for collaborative teaching and learning based on how we saw it enacted in elementary music classrooms. These considerations are summarized in Appendix E, as a way to convey our ideas about the implications and possibilities inherent in the collaborative elementary music classroom.

\section{Definitions of Collaboration Constructed by the CTSG: Introduction}

In this section, I describe the CTSG's collective definitions of collaboration as they emerged throughout the course of our meetings. (The evolution of each teacher's individual views on collaboration is documented in detail in Chapter Four.) The process of constructing our understanding of a collaborative elementary music classroom can be described as eventually converging on shared conceptual understandings.

Roschelle and Teasley (1995) describe an environment where people working together might come to shared conceptual understandings: "collaborative problem solving takes place in a negotiated and shared conceptual space, constructed through the external meditational framework of shared language, situation, and activity" (p. 70). The CTSG pursued a definition of collaboration in such an environment. Through our 
discussions we negotiated a conceptual understanding of collaboration, held in common, marked by shared language, and continually modified and revised as a group.

\section{Meeting One}

In our first meeting we discussed Karly's video. Collaboration was defined as involving all students in the mutual ownership of a musical effort, with minimal teacher presence, and an atmosphere of freedom to create without fear. These three components of our first definition are discussed below with examples and quotes.

We talked about how Karly asked all her students to stay musically connected. Andrea said, "she wants everyone to be involved, doing different things, but everyone's involved." We noticed that Karly accomplished large-group involvement by having the class sing the bass line while others sang solos, even though the end result was that the bass line was not as secure as if Karly had played it on the piano.

Karly brought up “ownership,” stating that a large percentage of students really felt that the class arrangement was "theirs." She said she wants, by the end of the year, to remove herself from the conductor role so students can be in charge.

We considered the social and emotional aspects of collaboration when Marlene wanted to know how students were inspired and motivated to do their best singing in memorized tunes and in on-the-spot improvisations. Andrea and Karly both suggested the key to this sort of freedom is an atmosphere free from fear of criticism by peers and teacher.

The last aspect of collaboration we discussed at our first meeting was how to engage the students Karly called "invisibles" in this atmosphere of involvement, ownership, and freedom. We defined invisible students as those who might not yet feel 
the ownership and pride of the rest of the class, but are not overtly disruptive. We agreed these students, who Andrea described as "scary" because they are just "sliding along," need to be gathered into the fold to participate in the rich collaborative experience. Based on our last comments of the meeting, I left with the sense that, as a group, we feel it is important to identify those students who seem truly musical involved and attached to the group effort, and try to increase the participation of those who are uninvolved. Meeting Two

At our next meeting on April 24, 2008, the topic of student engagement came up again when we watched Andrea's video. This time the full-group participation was facilitated quite differently. The goal was a student performance of a layered rhythmic canon in which the various parts were created and assigned by Andrea, not instigated by the students, as in Karly's video. We noticed the students stayed fully engaged, but instead of the minimal teacher presence Karly represented, Andrea actively directed the group step-by-step toward a positive end product. Marlene said, "[students] were amazingly attentive and there's a lot of excitement about each step... there's no dead time either." Karly said in this sort of collaboration, the role of the teacher is to keep the class going in a clear direction toward a musical goal.

This video depicted a different collaboration than we had defined the meeting before, when we saw students making up their own parts and eventually a class-created arrangement. Instead, Andrea taught the children their parts. She gave them specific directions about how to perform them. Here, the collaboration occurred when she elicited strategies from students about how to be musically in sync. She asked questions such as, "How do you stay together?" and "What are you thinking of to help you be with the 
group?" Students emerged as leaders and took on the role of conductor to help the groups end at the same time: "the perfect ending" was a goal urged by Andrea which the students pursued eagerly.

Karly characterized the collaboration in this scenario in terms of the children's eagerness to share what they thought. She said, "they were excited to share their strategies and what they heard." Marlene agreed, saying, "the students had good ideas, and Andrea used them."

The idea of social and emotional collaboration arose, as we observed an atmosphere of friendly helpfulness among the students. Karly commented on how Andrea "made sure to make the students feel as if they're in a community." Marlene said that was the strongest aspect of collaboration in the video: the students' ability to listen and be friendly. She said, “They just listen to each other. By not talking when she's talking, but not complaining, or whining...they follow directions...they're just together."

We agreed that while Andrea was definitely a leading presence in the class, the atmosphere seemed collaborative because the students shared her goals. Andrea said:

They want to get to the next step, and you wanted to know how I got them excited to get to that next level? It's how I present it... I can get them excited, and they want those challenges, they love it, they want to make each time through better and they know I can help them do it, and then they say, "Can we try something else? Harder?" (Andrea, CTSG meeting two)

I said I had learned from this video that a classroom could still be collaborative even if the teacher creates the arrangement, and is actively directing the students through the steps to get there. The collaboration results from the students sharing the goal and continually persevering in the process of reaching the goal. 
This definition of a collaborative classroom was a change in my conception from meeting one. I left the first meeting feeling students collaborate best when the directions from the teacher are minimized and the students create their own parts. However, Karly helped bridge the views of collaboration in meetings one and two for me when she summarized what she had learned. She said that collaboration can happen naturally when the end goal is ultimately a musical product, and that perhaps the teacher does not need to try so hard to purposefully facilitate it if the musical product is appropriate:

At the beginning of this project I was thinking, okay I have to have the kids collaborate. What strategy can I use to have them collaborate? How can I make them collaborate? But now I realize, when you make music, you collaborate! You automatically do. And if you foster musicality in your classroom, if your end goal is something really beautiful and musical, then you are using collaboration, and the kids are naturally collaborating. I just think it's so cool that [in Andrea's video] it wasn't a big deal, it was just class, and you were just making music, and that was the most important thing. (Karly, CTSG meeting two)

So when the students worked to make their music better, they collaborated, even though the teacher was highly involved in getting them to each next stage. Marlene agreed, defining natural musical collaboration in terms of the demands of musical parts:

“They're working together. And you have to, in order to keep those two ostinati together so it doesn't become a train wreck. You have to be listening to each other." However, Marlene also pointed out that merely putting students in an environment where they have to combine two parts was no guarantee that successful musical collaboration could occur. She cautioned, “it doesn't just happen. And Andrea was saying a poem on top of [the ostinati] so it could be confusing. But they were really listening." Beginnings of a Socially Constructed Definition

In these two meetings, a socially constructed definition of collaboration was starting to emerge. Out of the four separate conceptions we brought to the first meeting, 
we converged on the idea that collaboration in the elementary music room involves all students in the musical process, and that students need to feel free to offer input.

At the first meeting, one area in which my definition may have diverged from the other members is in the role of the teacher. Neither Andrea's nor Marlene's remarks indicated they believed, as I did, that the teacher in a collaborative classroom does less direct instruction and more gentle facilitation. I believe Karly shared this idea, as that is how she enacted her role of teacher in the video she brought. She also made statements throughout the meeting that confirmed that viewpoint.

In our second meeting the four of us agreed again. We all marveled at Andrea's remarkably engaged students, and asked a number of times how she established such strong student buy-in. We admired Andrea's manner with the class; the way she took students seamlessly from one step to the next challenge implied planning, management, and effective leadership. We did not, however, find her dictatorial. In fact, I said, "it was great classroom management but I didn't get the sense that the kids were being managed: your management was of the time and the activity."

The positive comments we made about Andrea's video point to our growing understanding that the teacher may be quite directive in a collaborative music classroom. In the April 24, 2008 meeting, after watching Andrea's video, we defined collaboration as a joint student-teacher effort to move toward mutually shared musical goals. We saw that a teacher's strong presence in the front of the room, leading the group, does not preclude a collaborative atmosphere. If students participate in making the end product musical, their contributions toward that end will likely be collaborative. We all perceived the students' excitement on video. We attributed that enthusiasm to a goal shared with 
their teacher: to make their rhythmic canon crisply accurate and end with a concerted flourish.

One divergence in our understanding of student musical collaboration concerned the amount of student creativity in the lesson. Karly said the activity on video seemed familiar to the students, so she wondered if Andrea could next try letting students improvise. She speculated this would involve students in "conversing with each other and going to the next step of collaboration." Karly also wondered if adding a student-created element could enhance "buy-in:" “Then it's really their tune, it's like they're creating it together so they have that personalization, their stamp on it...that piece can be so powerful to kids." From these comments, I understand that Karly perceived student creativity as an important part of collaboration: creativity was not a salient part of Andrea's lesson.

\section{Meeting Three}

In our third meeting, we talked about the collaboration that seemed to be happening among the students in Marlene's class. Because Marlene has to go into the students' classrooms to teach them music, the video showed her teaching students sitting at desks, arranged into groups of three to five.

We identified collaboration in students turning their bodies, heads, and ears to listen and play together, compensating for a less-than-ideal room arrangement that didn't let them easily look at or hear each other. We called this "working together," a phenomenon which was notable to us because initially we had not seen much possibility for collaboration in that room. 
I was surprised how often students on the video turned in their chairs to look at each other. Karly said, "I noticed that too, and I wondered if they were listening to each other, like the kid in the front row, he turned his ear more than his body. He was trying to hear what the other people were playing and blend his sound and play with the others."

Andrea was the first to call this "working together:"

We all noticed when two recorder players were playing [a duet] that they were watching each other, they were really making sure they did the same fingering at the same time and were playing right together. They are working together and working together to listen...bouncing to the beat, doing whatever they needed to stay working together. (Andrea, CTSG meeting three)

Karly pointed out that the "working together" phenomenon happened even when students were far across the room from one another. Andrea said that often students were looking around "to see who else was putting their fingers down with them, or if they were completely off or wrong...to see what the others were doing and they were bobbing along together." We noticed in addition to turning in their chairs, students who were working together with others across the room also tilted their heads, to turn their ears in a certain direction. Karly was the first to notice that behavior: "I love that because that's what musicians do, you don't always look right at the person. Drummers in their ensembles turn their heads like this."

Students turning their bodies, heads, and eyes to watch and listen to each other seemed to indicate their strong desire to be together and in sync. While they might not actually be able to hear other students playing recorder across an acoustically dead classroom with many desks and bodies in the way, they could certainly look at one another to make sure their physical movements were coordinated. We defined this aspect of collaboration as similar to the natural collaboration we had witnessed in Andrea's 
room: collaboration that evolves naturally from students' strong desire to make a musical ensemble work.

We pointed out a number of times on video when students were working together. We noticed Marlene's positively reinforcing the collaborative behaviors she wanted to see. However, Marlene said it was difficult to foster student perseverance toward a musical goal:

They want to get it the first time, and they want it to be easy, and if they don't they start saying, "This is hard, I cannot do it, it's too hard" and the whole chemistry of the room changes because the others start thinking, "They're right, this is too hard" and they just stop doing, they don't keep trying, and they don't interact anymore, they just go into their own spaces. (Marlene, CTSG meeting three)

The CSTG members identified another form of collaboration on the videostudent interaction in their table groups - that Marlene could encourage. We saw students watching and working side-by-side. We speculated that harnessing that small group dynamic would help more students work toward a musical goal and stay musically involved.

Karly said:

Maybe use the strength of [students at] each table. Someone who's got one part or the whole tune down, that child can take on the role of leader or teacher in the whole group, and have everyone finger along or sing along so they stay engaged. (Karly, CTSG meeting three)

We agreed that students should turn to one another, to talk and process information together. We also decided that if students were allowed and prompted to talk to each other, more of them would stay engaged. This portion of our discussion highlights the continued emergence of a shared view: collaboration equals whole-class involvement. 
For the first time in our meetings, we connected whole-class involvement with the possibility of an outwardly chaotic environment. Marlene said she felt most comfortable and effective when students were playing and she could just sing along. At several points in the lesson she was even singing the instructions to the students, to keep the starting pitch in their minds. They laughed and joined in on the pitches with her. Even though there was talking and off-task behavior, she was happy, saying,

If half of them are playing the melody, that's an accomplishment, if I can hear the melody over the [crowd noises] that's an accomplishment, if I don't have to waste time managing and disciplining, that's an accomplishment. It's crazy sometimes but I can see that they're learning, it's their style. They need to talk, they need to enjoy it. It should be about them, not me. (Marlene, CTSG meeting three)

However she told us that her mentor and principal were interested in crowd control, a concept she found difficult to realize in action. Marlene said she wants a classroom with more student choice, which means "sometimes you're going to have a mess... where you give them freedom, to do more creating, there's going to going to be talking." Andrea agreed, saying that working together is loud:

Just put them in pairs and say, "You're the teacher, and you're the student." The kids take such ownership of that! Yeah it gets noisy and yeah it's another thing where your principal might come in and ask, "Why is it so noisy in here?" But that's when they're working together! That's when they're creating their stuff! (Andrea, CTSG meeting three)

Marlene said she wanted to try lessons in which students could create more freely. She reminded us she felt uneasy about her students' ability to work together appropriately. We acknowledged what we had seen on video: this class did have a tendency to be distracted. Marlene said the distracting behavior sometimes turned hurtful and counter-productive; she had to figure out ways to get a happy medium between 
control and freedom. We all understood this delicate balance in elementary music. We spent a few moments wondering if and how productive collaboration could be taught.

One aspect of Marlene's classroom that did embody freedom and choice was the solo and duet performance portion. Students in her class were eager to perform. Not only were they enthusiastic soloists in the front of the room, they loved picking partners from across the room. We decided this was a matter of enabling student musical choice: they picked students they were on the same playing level with, students they admired, or their close friends.

The solo and duets on video brought to the forefront of our discussion the idea of student freedom and risk-taking. We told Marlene it was obvious she has established an atmosphere in which students can rely on each other and are not afraid to play for one another. Karly said students were motivated to work together well in this instance because Marlene gave them space to show off their hard work.

In this meeting, we continued to refine two previously identified conceptions of collaboration as (a) encompassing whole-group involvement, and (b) naturally occurring when students work toward a goal of musical ensemble. We confirmed our notion that collaboration between students is aided by an atmosphere encouraging freedom, choice, and risk-taking.

We added two components to our definition. The first is the idea that students' eye contact, body positioning and body language are markers of collaboration. This evidence might be overlooked by teachers, especially when we insist that students face forward and watch the teacher. 
Second, we wrestled with the idea that collaboration in the music classroom might necessitate chaos and noise: side effects that other teachers, administrators, and even students might be uncomfortable with. Our language in this part of the discussion reflects ambivalence; no one wanted Marlene to manage her class to the extent that there was no freedom of self-expression and student choice left. On the other hand, we understood the difficulties that teachers face when letting go and allowing students to collaborate more freely. We wondered if skills for peaceful, productive collaboration could be taught.

\section{Meeting Four}

At the fourth CTSG meeting on May 9, 2008, Karly presented again. We tried to answer the question that was asked the last week: how can students be taught to collaborate productively?

Karly's video featured students going through a sequence to reinforce the underlying harmonies of "Hava Nagila" in order to create improvisations on the song. We noticed that Karly had a specific order for these steps: first she gave students practice time to sing and audiate the tune, which they knew well. Then students got with a partner, sitting knee-to-knee, and sang the song to each other in unison, blending their voices. Next they were instructed to take turns singing the bass line while their partner sang the melody. Finally Karly told them to take turns improvising four-bar phrases to their partner, question-answer style, while she sang the bass line. The students then took up drums and tambourines so they could dance and sing together.

We noticed elements similar to Andrea and Marlene's teaching. Karly was moving her students along in a directed, stepwise way. I said she kept everyone focused on the goal of playing and singing all together at the end, saying things like, "the next 
thing we're going to do is," "the next level is," and "we're going to get to the dance at the end." Previewing upcoming steps was a technique we had seen clearly from Andrea. Andrea said, "She highlighted the next step for them. It was definitely sequential: first the song, then the bass line, then the improv, then they added the dance and the instruments. It was very stepwise and sequential, which was nice.” In directing her students, Karly had adopted Marlene's practice of singing the directions on the starting pitch or on tonic. This had the effect of pulling the students along quickly, with less space in which students would talk. When we saw these elements, we said, "Oh, that's Andrea!" and "Oh, that's Marlene!"

We commented on how smooth and rhythmic the dance was at the end; we had all had problems with students pulling roughly against the beat in this sort of circle dance. I said the students were collaborating to make the dance work, but only because of the steps they had gone through to get there:

Part of the reason that circle happened so rhythmically was because of the work done originally. (Andrea: Yes. the process). The process with the audiation, and the feeling the phrases in the improvisations: it was clear everybody was subdividing the beat all over their body, so I don't think they had a tendency to pull in the circle. (Ann Marie, CTSG meeting four)

We also noticed how some of the partner-pairs on video were more successful than others at working together. We wondered if they were assigned partners, how they learned to work together, and if the intimacy of singing face-to-face helped them or intimidated them. We wanted to know what tools the partners used to decide who would go first. We wanted to know if there was a "taxonomy" (my word) of collaboration: a specific order in which collaborative activities might be organized for optimum success. 
These questions signaled a change in our dialogue. In this meeting there was less talk related to constructing and negotiating our group's definitions of collaboration. We came to an unspoken conclusion that what we had seen on video was indeed collaboration, and proceeded to unpack Karly's steps in teaching it. It was clear the students were accomplishing things we admired, and we wanted to take apart the elements to understand better. At the same time, our discussion reinforced our previous understandings about the characteristics of elementary music collaboration: full-group involvement, freedom of choice, risk-taking in a safe atmosphere, and musical goal setting and sharing.

Karly said having partners audiate together first, blend their voices in unison, and then improvise back-and-forth is an important aspect of learning collaboration. She said it was important for students to understand how to stay together mentally, when they needed to match one another, and when they were free to personalize the music, so she starts these practices in kindergarten. The developmental continuum of collaboration is something Karly likes to watch unfold:

In second grade they're just starting to get out of that mindset, "it's mine, it's mine" and they're able to share and listen more. In kindergarten, "it's all about me" but in second grade they really love creating and sharing it. Just because they're singing a tune, in tune, doesn't mean they really understand the tune, so I'm trying to really get at what they know in these types of activities. (Karly, CTSG meeting four)

Andrea agreed blending voices and improvising with partners contributes to the safe atmosphere of learning. However, both Andrea and Marlene said that partners seemed more self-conscious blending than in taking turns. We thought they needed more experiences singing all together as a group, so that singing in unison with only one other person would not frighten them. Andrea said: 
I would have thought that singing together would be like [snaps] done! but you're [Marlene] right, that seemed to be difficult. I wonder if they weren't with partners and just sang it together first, would that have made things more in a comfort level? Maybe in improvising they had a chance to think while the other person sang, but then when they were blending they were, "Oh geez, we're singing the exact same thing at the exact same time." (Andrea, CTSG meeting four)

Marlene agreed, saying in the "blending voices" portion, students might feel like it's more obvious if they don't know their part. She was also concerned about what happens if one of them doesn't get it right: "The other one maybe says, 'He messed up!' or 'he's messing me up!'”

Karly said she always wants active learning, which for her involves the students turning to each other, checking for understanding, and engaging all students, often in small groups. Karly said that in collaborative pairs, students will remember their partner's ideas: "I could tell them things a million times, but in this case, their friend told them, so they'll remember it."

We decided the optimal sequence would be to have the students sing in unison first, learning the song and instrumental parts well and experimenting to find their preferred part. The next step could be working in pairs to blend and practice improvising. Later they could work in small groups to perform parts of the dance and arrangement, ending with a whole-group performance.

Our discussion returned to several common themes. First we discussed student choice. We had talked about student choice in meeting one, in terms of how students selected their own instruments and made up their own parts. In meeting three, we identified situations when students chose who to perform with. Now, in this meeting, student choice was student self-determination, leading to a real feeling of ownership. Students themselves determined that they were good at certain parts, and gravitated there: 
a process of ownership Karly encouraged as being akin to what adult musicians might do.

She said:

They all learn all the parts. But some of the classes just take things upon themselves to assign. They have set parts for certain tunes, that instrument's theirs. If everyone in the class feels like Joey's part is the maracas, then he will really own that. He is an instrumentalist, a professional maracas player right then! In this case, I said I need three tambourine players and some said, "Okay that's my part." But others said, "I'm really good at singing for this tune. That's my strength." And I tell them that too, "Anthony, you're like a real drummer, good at playing off-beats, you should do tambourine on this tune." (Karly, CTSG meeting four)

Karly knows her students' strengths, and part of collaboration for her is allowing

for enough experimentation to find them. We noticed that Karly doesn't seem to try hard to control this process, she lets it happen naturally, and lets students feel they have responsibility for an important part of the puzzle. However, Marlene pointed out the difficulty of finding time for equitable amounts of experimentation, and in helping students learn a part they want to play but is hard for them. First she asked, "Do you ever get one kid that wants to do something that he cannot do?"

Karly said that she reassures students they'll get to rotate through all the parts. Marlene reminded us how challenging that is to manage: "In 30 minutes? So hard to do! And then remembering who didn't get to play the drum, and give that person a chance for next time?" She said she had been told to keep track in writing of who got to try what part each week.

I pointed out that I did not like the view of teacher as the all-powerful one who has the ability to dole out the parts utterly fairly; that this was an unrealistic expectation for students to have of their teacher, and one likely to be unfulfilled. Andrea said that she wants students to understand that making music is something that involves working 
together for the greater good, and that everyone shares responsibility for helping students learn and experience a variety of parts.

This shared responsibility, working together, was defined by Andrea for the first time in our meetings as "community:"

That's just the reality of music. Music is community. Unless you're a piano soloist, you need to work together, you need to have that community feel. The nature of our classes should be that everybody has a part, like the dance, the tambourine. Kids need to know everyone has a part in class, everyone has a part in music, they all fit together and one being missing is a big chunk that's gone. (Andrea, CTSG meeting four)

Our definition of collaboration was definitely converging around a few key points. After this meeting, we seemed to agree that collaboration in elementary school music could be taught, and that sequencing of steps matters. We agreed students should find their strengths and feel part of a community.

I felt there was a divergence in views that might or might not be repaired at our next meeting. I thought everyone understood the value of group ownership, student choice, and the goal of a music-maker community. But I sensed Marlene was still grappling with how to implement those values in her current context. I felt her questions in this meeting were much more in the "How do I do collaboration?" vein, as opposed to "What does collaboration look like, for you and for me?"

However, at this meeting, Marlene shared a success story about implementing a small-group collaboration lesson in her classroom this week. (This anecdote is detailed in Marlene's Story, Chapter Four.) Marlene's confidence and pride in the community she was able to establish made me think that she was having success with many of our group's ideas, even if she was wondering how to do more. 


\section{Meeting Five}

At the May 22, 2008 meeting, Andrea brought a tape of students learning to sing and move to a round. As we watched her film, we commented on the way she sequenced the events of the lesson, much like she had shown us on April 24. We noticed a fine shade of distinction, one perhaps only obvious to teachers like us striving to understand collaboration in detail.

Andrea was still scaffolding the lesson in the sense that she took the students to increasingly more complicated levels: a two-part round, then three parts, then four, with accompanying movements in concentric circles changing directions at phrase endings. Karly described a difference in Andrea's manner on this video: "I saw a gradual release of responsibility on her part: she stopped singing and started snapping, then she stopped snapping, saying, 'I'm done leading it, you don't need me'" (Karly, CTSG meeting five). Rather than controlling the performance more when the level of musical challenge was highest, Andrea did the opposite and removed herself. We said this increased the students' self-reliance in achieving a shared goal of musical ensemble. Karly said, "[students] kind of scaffolded their own learning."

In the first meeting we defined the collaborative classroom as one with a large percentage of students who buy into the process. In this meeting we continued to refine our ideas about student ownership, this time connecting it to students' ability to give input and feedback. Andrea listed two things she wished she had done in the class segment on video:

The feedback process: that's one thing I wish I could change. After they were rushing like mad, I stopped and told them: you guys were rushing! I should have asked them what happened...I should have had them turn to their neighbor and 
talk about what was happening and what would help them make it better. (Andrea, CTSG meeting five)

We seized upon the idea of students offering self-evaluation as a vital part of collaboration. We theorized that one positive aspect of this type of collaboration is the window into students' musical thinking that might open up for teachers. For instance, we talked about one boy on the video who had the chance to ask that the groups stop stomping loudly to stay together. He appeared to be bothered by the loud noise. In fact, when the groups performed the round softly or were silently audiating, the ensemble was much better.

Karly said, "He was really trying to help out the group but also help himself out. He was suggesting a different strategy for having them all collaborate in a creative, good performance, a strategy that made sense." Marlene pointed out that this event gave Andrea important information about him: "We may have to investigate, what is his background? He may have an issue where really loud noises bother him."

Sure enough, Andrea told us later this student was sensitive to noise, and that his ability to make that suggestion led the class to consider how louder was not always better. It led her to think about allowing more students to self-determine the best ways to learn: "for him, specifically, it really helped him to be able to sing it in his head without any excess noise, to focus on what was in his head."

In this meeting we asked six different times about how to incorporate student selfassessment. We asked questions like, "How can we get kids involved in the feedback process?" (Ann Marie) and "what is going on in their minds and do they have feedback to offer to the group or themselves?" (Karly). Andrea said that getting her students to 
verbalize or demonstrate their own questions and problems would be a future goal of hers.

We continued to converge around the idea that student musical collaboration can be taught. We talked the ways Andrea taught good ensemble and collaborating behaviors. We listed things we heard her say, from tangible suggestions like "if you watch your classmates you can walk to the beat they are" to larger ideas about collaboration: "if we all do it, if we all think it together, we'll have success." Karly defined the resulting collaboration among students as, by the end, "looking like everyone was on a more even playing field: thinking less about themselves and more about everyone around them, through the use of their own tools."

Marlene continued to define collaboration through her own lens, one that led her to fix upon the students' cooperation, kindness, and respect of the teacher. I believe this line of questioning persisted for Marlene because of her continued concern that her students simply do not function as a group, even on a basic level. She said:

I hate to ask this week, again! [laughs] But how long does it take to get there? To have a group of kids doing what you're asking? They're collaborating with each other now, for sure. Does it take a year, two years, I wonder? Maybe it depends on the class? (Marlene, CTSG meeting five)

She highlighted the importance of trust, saying:

My kids still don't have a lot of trust. I feel like it has taken me longer than ever, this year, but finally they are trusting and understanding me more, but still, you can tell they trust you, they understand you, Andrea. They don't ever question "why are we doing this?" (Marlene, CTSG meeting five)

Karly reassured Marlene that her students question her as well, sometimes obnoxiously. This took us into a discussion of collaboration as an important life skill. Marlene said she felt if she could teach her students to collaborate she would feel like she 
did something very important. Karly and Andrea agreed, saying that it is important for music teachers to realize that they are teaching young children, not just the content of music.

In this meeting, our definition of collaboration expanded in two ways. First, we described collaboration marked by student input and self evaluation as a tool: one that gives teachers new information about students. Second, we labeled collaboration as a life skill that in some cases might supersede musical content in importance.

I feel this second expanded view of collaboration finally helped us converge around an understanding of collaboration that better encompassed Marlene's particular situation. Marlene asked questions about how to establish an atmosphere where collaboration could occur, and she consistently identified positive student social behavior as collaboration. Including more than just musical elements in our definition of collaboration made it more complete. If collaboration is a life skill, we might see evidence of it in students sharing, being respectful, caring, listening, and not hurting themselves or others.

We also maintained and strengthened our shared idea that students working toward a musical goal would probably naturally collaborate. We saw that Andrea's gradual retreat from the director role resulted in students rising to the occasion to do harder musical tasks, better. The idea of ownership came up again, this time with the caveat that students asked to identify problems and suggest solutions may be more attached to the process. Finally, we confirmed our previous conception that collaboration may be taught, and identified more possibilities for doing so. 


\section{Meeting Six}

On May 29, 2008 the CTSG met to watch a video from Marlene's classroom.

Andrea was unable to attend due to an emergency.

We saw Marlene had the students working in groups of four or five, using the grouped desks to separate the teams. We noticed the students were all engaged, pointing and gesturing over the paper. We noticed excitement in the groups, organized turntaking, and students watching closely even when it was not their turn to write. We heard a lot of talking but identified it as on-task, musical chatter. We saw groups figuring out various ways to decide who would present their work to the whole class: one group armwrestled for the honor while two others played rock-paper-scissors. Karly said, "They worked it out themselves in a pretty mature way. There were many other options they could have taken, including fighting, and they didn't."

We noticed the students quieted down when the groups came forward to present their work. I said it was probably crucial in this collaborative environment to have the students come up and explain their product. Karly agreed, saying, "Yes, as opposed to just Marlene standing up there, or posting them as the kids leave."

We observed the physical evidence of student engagement in the groups.

I said the students moved themselves around to be more than bystanders: "They could've all just been sitting there in their seats but they made the extra physical effort to stand, and peer over, or even just to get up and walk over so that they could see [the paper]."

Marlene said she had a revelation over the last week:

I think these kids are more relaxed when they're in groups. I think it's selfish of me to think that they should be quiet all the time because if someone walks in, it's going to look good on me. That's just ego, that "I want this to look perfect if someone walks in and sees it." I decided I can take the noise and the mess if 
they're learning and if it's a valuable thing they're doing. (Marlene, CTSG meeting six)

I said Marlene looked more relaxed on this video. She said, "Oh my. That's how it looked, but inside I was anxious, like, oh my God, it's a mess." We talked about the difference between learning and a mess. Karly said the end result was proof enough: "They were having fun and they were learning a ton! And they now know how to read, write, and play that tune, and they were in control the whole time. If you needed to get their attention, you could."

In this meeting Marlene, Karly and I reached an understanding of collaboration in Marlene's class. Instead of asking the other teachers how they established a collaborative atmosphere, Marlene described how collaboration looked for her students. She acknowledged the need to look like she had the perfect, well-behaved class, but stated that she would do what it took to keep her students relaxed and learning. We converged around the idea that three elements of collaboration are crucial for Marlene's setting: students' ability to talk and move around, model their thinking for their peers, and share their accomplishment with the class. We supported Marlene in the idea that although these processes might not look tidy to outsiders, she was doing the right thing to foster collaboration.

\section{Wrap-Up Meeting}

For our wrap-up meeting on June 11, I pulled together a handout from the transcripts and notes from each meeting. The CTSG teachers wanted a summary of things we had talked about for their future reference. Making the handout was a good exercise for me; I took a holistic look back over the course of our discussions and made an initial foray into writing what we learned as a group. 
At this meeting we did not change anything in our socially shared understanding of collaboration. Instead, we summarized what we had learned about collaboration in elementary music. Our conversation centered around five topics: (a) community; (b) the role of the teacher; (c) what collaboration is not; (d) how to explain collaboration to other teachers; and (e) the role of collaboration in learning.

Community. The first heading on the handout was "What forms does collaboration take within the elementary school classroom?" To answer this question, I listed three points which had recurred at each meeting: (a) making music together in a musical way that requires listening and interaction; (b) working together with peers and teacher on a task; and (c) learning the sensitivity and awareness necessary to function as a musical entity.

Under these broad headings I listed the musical activities from all the videos that seemed to illustrate these concepts: things like creating arrangements as a class, improvising and elaborating over known parts when peers are maintaining the known part, playing recorder duets to help each other learn, and movements performed with others in small and large groups. Karly identified a commonality in this section of the handout:

Every bullet [under the first heading] talks about working together, in the sense of community. It's not just about learning as an individual. It's working as a team, helping one another learn, performing with others. That's kind of what I assumed it would be, but it's kind of neat to remember that it was the same, across all of the videos and across all of our thinking. (Karly, CTSG wrap-up meeting)

Other than the above quote, collaboration as defined by community was not mentioned. We talked about specific collaborative activities seen on video, such as confirming how various songs went, and sharing what we remembered from particular 
classes. The CTSG members accepted my handout examples, and spoke a little about how these activities could be practically carried out.

The role of the teacher. Upon reading the handout, Marlene said she was trying to use more strategies to facilitate collaboration. She said an important one for her was stepping back to let the students have more control, which she described as "taking a breath" (Marlene, CTSG wrap-up meeting). Andrea asked what had happened in meeting six to give Marlene this idea, and we all helped fill her in on how we had decided last week that collaboration can be facilitated by Marlene in her own classroom. Andrea was pleased to hear about the last week's discussion and she congratulated Marlene on trying the "Hot Cross Buns" lesson.

Karly also alluded to the ease of the teacher in facilitating a collaborative classroom. She said, “it's the comfort level of the teacher in how we're all kind of taking a chance, when you let the students collaborate. You have to remain in control in a way, then you step back" (Karly, CTSG wrap-up meeting).

Andrea agreed, reminding us that her principal had advised her to let the students teach themselves more, and ask them open-ended questions with no predictable answer. For Karly the teacher should listen and allow collaboration: "I'm going to listen and take it in when they suggest something. I'm not going to just say, okay we're moving on. I'm being aware of children trying to interact with each other" (Karly, CTSG wrap-up meeting).

Again, in this portion of our meeting, we did not talk about defining collaboration more specifically. We talked about how teachers provide space for it and how questioning and openness seem to instigate collaborative interactions. 
What collaboration is not. I asked what is not collaborative in elementary music classrooms. Andrea said sitting at desks doing worksheets, although even that activity could be altered to be more collaborative: "I guess it could be, if the teacher said "okay, share your answers with your next door neighbor," that could be termed collaborative. But if you have your own packet and are writing down all your own answers, no" (Andrea, CTSG wrap-up meeting).

Karly defined non-collaboration as "anything that does not involve interaction. I've heard that word in here a lot. If they kids are interacting with the teacher, and conversing, and learning, with her, they're collaborating...But drilling something? That's not collaborative in any way" (Karly, CTSG wrap-up meeting).

We discussed the pressure of conducting an ensemble and preparing for concerts, and how those forces often made it hard to be collaborative. Andrea said due to schedule pressure in her chorus rehearsals, she was likely to tell students they were wrong and how to fix it: "I don't think I ever stopped to ask them. I don't think I opened it up to the floor, to ask, "What is a way we could make that better?" (Andrea, CTSG wrap-up meeting).

Explaining collaboration to others. We talked about how teachers might be wary of collaboration for a number of reasons, especially if they have not thought much about all the things collaboration might imply for elementary music. Karly said, "I can imagine not having this intensive study, the CTSG, and you're [Ann Marie] giving a workshop, and you say these things to them and people go, [sharp intake of breath] 'No way!'”

Andrea said teachers would first wonder, "Collaboration? What does that even mean?" She said that giving other teachers examples of small collaborative efforts, like "think-pair-share" would be helpful. She said teachers might be fearful, because they 
haven't been taught to do collaborative projects; she likened fear of collaboration to the fear of musical improvisation some teachers have. I disagreed that collaboration would be scary to teachers. I reminded Andrea of her student-teacher collaboration video at our May 22 meeting:

You were facilitating decisions by stepping in when you need to. But you were not a dictator! You asked the kids so many times, "how can we do this differently?" or "how do you hear the people on the other parts?" But there was never a minute in looking at your video when I think even the most controloriented teacher would get freaked out. So it's almost like a mentality, more than it is a drastic change. (Ann Marie, CTSG wrap-up meeting)

The CTSG teachers agreed with that idea. Marlene said the "collaborative mentality" can simply mean "putting [students] in a place where they might collaborate, but not needing to run it, as the boss" (Marlene, CTSG wrap-up meeting).

Andrea suggested portraying collaboration as occurring across a continuum. Marlene agreed, saying that small steps can make teachers more comfortable. Karly pointed out that teachers could be told that once they have under their belts strategies for facilitating collaboration, they could try new ones. However Andrea pointed out that there is not a hierarchy of easy and hard collaboration:

That's important for teachers to realize. It's not like, 'oh I've graduated from think-pair-share, I can't do that anymore with my class, I'm on to harder collaborative things"... It should be if you're, like we are, really comfortable with collaborating, it's still okay to do a think-pair-share! (Andrea, CTSG wrap-up meeting)

Collaboration and learning. I asked the CTSG members if we had evidence that collaboration enhances learning. They all talked about how collaboration gives teachers a fresh look at what their students might be thinking. Marlene said through collaborative activities, she saw her students relax: "There's less pressure. They might feel like they can learn better if they don't have to perform in the typical way, alone, unsupported" 
(Marlene, CTSG wrap-up meeting). Karly said collaboration may not enhance learning, but it is one way to find evidence of learning. She said:

Hearing what they say, watching what they do, independent of you but with each other, it's really a way of understanding what's going on in the environment, and being able to respond to it better than if you just heard one kid alone. (Karly, CTSG wrap-up meeting)

Summary of wrap-up meeting. I cannot identify data from this meeting that confirm my premise going in: that this summary meeting would be the final step in solidifying a definition of collaboration constructed and collectively held by us. Instead I identified two larger characteristics of collaboration we summed up.

First, it is clear that we have a mutual belief in the importance of the teacher in creating space for collaboration. We identified a number of ways teachers might be encouraged or led toward establishing this space in their classroom, but we agreed that teachers' comfort level with these ideas might vary. Placing collaborative classroom strategies on a continuum might help teachers.

Second, we re-stated the idea that collaboration in the classroom can be a valuable way to examine the learning of students. We identified a number of situations in which collaboration would not occur; chief among these are rehearsals in which the director does not ask for student input, and individual activities like worksheets.

\section{A Socially Constructed and Shared Definition of Collaboration}

Figure 6.1 summarizes how the group's shared understanding of collaboration materialized. 
Figure 6.1

Characteristics of Elementary Music Student Collaboration as Defined by CTSG

\begin{tabular}{|c|c|c|c|c|}
\hline \multirow[t]{2}{*}{ Date } & \multirow[t]{2}{*}{ Presenter } & \multirow[t]{2}{*}{ Theme } & \multicolumn{2}{|c|}{ Defining Characteristics } \\
\hline & & & Students & Teachers \\
\hline \multirow[t]{2}{*}{$\begin{array}{l}\text { April 4, } \\
2008\end{array}$} & Karly & $\begin{array}{l}\text { Students } \\
\text { Collaborate }\end{array}$ & $\begin{array}{l}\text { Create, share, join } \\
\text { their musical ideas }\end{array}$ & Facilitates student creativity \\
\hline & & & $\begin{array}{l}\text { - All students } \\
\text { musically involved } \\
\text { - Feel ownership of } \\
\text { product }\end{array}$ & $\begin{array}{l}\text {-Teacher direction is minimized } \\
\text { in favor of student freedom and } \\
\text { suggestions } \\
\text { - Atmosphere of creativity, } \\
\text { verbalization of personal choice } \\
\text { without fear } \\
\text { - "Invisible students" identified } \\
\text { and connected with }\end{array}$ \\
\hline \multirow[t]{2}{*}{$\begin{array}{l}\text { April } \\
24, \\
2008\end{array}$} & Andrea & $\begin{array}{l}\text { Teacher } \\
\text { Directs } \\
\text { Collaboration } \\
\text { Toward }\end{array}$ & $\begin{array}{l}\text { Listen, help, and } \\
\text { share }\end{array}$ & $\begin{array}{l}\text { Teaches parts, elicits student } \\
\text { ideas for improvement, and } \\
\text { offers a musical experience }\end{array}$ \\
\hline & & Musical Goal & $\begin{array}{l}\text { - Are musically } \\
\text { involved at all times } \\
\text {-Share end goal with } \\
\text { teacher } \\
\text { - Make suggestions } \\
\text { for improvement in } \\
\text { friendly and helpful } \\
\text { ways }\end{array}$ & $\begin{array}{l}\text {-Explicitly instructs ensemble } \\
\text { behaviors } \\
\text { - Facilitates student movement } \\
\text { toward end goal } \\
\text { - Provides space for music- } \\
\text { making in which collaboration } \\
\text { might happen naturally }\end{array}$ \\
\hline \multirow[t]{2}{*}{$\begin{array}{l}\text { May 1, } \\
2008\end{array}$} & Marlene & $\begin{array}{l}\text { Students } \\
\text { Regulate Own } \\
\text { Collaboration }\end{array}$ & $\begin{array}{l}\text { Interact socially and } \\
\text { musically }\end{array}$ & Allows natural collaboration \\
\hline & & & $\begin{array}{l}\text {-Are musically } \\
\text { involved at all times } \\
\text { - Become visually } \\
\text { aware of each other } \\
\text { - Turn heads and } \\
\text { ears toward each } \\
\text { other to listen better } \\
\text { - Choose partners for } \\
\text { duets based on social } \\
\text { and musical factors }\end{array}$ & $\begin{array}{l}\text { - Reinforces collaboration by } \\
\text { allowing turning in seats and } \\
\text { student choice in interaction } \\
\text { - Teacher allows chaos and noise } \\
\text { in the service of collaboration } \\
\text { - Teacher provides space for } \\
\text { music-making in which } \\
\text { collaboration might happen } \\
\text { naturally }\end{array}$ \\
\hline
\end{tabular}


Figure 6.1 (continued)

\begin{tabular}{|c|c|c|c|c|}
\hline \multirow{2}{*}{$\begin{array}{l}\text { Date } \\
\text { May 9, } \\
2008\end{array}$} & Presenter & Theme & \multicolumn{2}{|c|}{ Defining Characteristics } \\
\hline & Karly & $\begin{array}{l}\text { Teaching } \\
\text { Collaboration }\end{array}$ & \begin{tabular}{l}
\multicolumn{1}{c}{ Students } \\
Interact socially and \\
musically
\end{tabular} & $\begin{array}{l}\text { Teachers } \\
\text { Consciously teaches collaborative } \\
\text { skills }\end{array}$ \\
\hline & & & $\begin{array}{l}\text { - All students are } \\
\text { musically involved at } \\
\text { all times } \\
\text { - Interact with partners, } \\
\text { small groups, and } \\
\text { whole class } \\
\text { - Identify own and } \\
\text { others' strengths } \\
\text { - Feel part of a musical } \\
\text { community }\end{array}$ & $\begin{array}{l}\text { - Enables collaboration through } \\
\text { logical steps } \\
\text { - Enables collaboration in full } \\
\text { group unison, then pairs, then full } \\
\text { group performing different parts } \\
\text { (whole-part-whole) } \\
\text {-Allows time for students to } \\
\text { experiment and find strengths }\end{array}$ \\
\hline $\begin{array}{l}\text { May } \\
22 \\
2008\end{array}$ & Andrea & $\begin{array}{l}\text { Collaboration } \\
\text { and Self- } \\
\text { Assessment }\end{array}$ & $\begin{array}{l}\text { As a group are self- } \\
\text { reliant } \\
\text { - Rely on each other to } \\
\text { meet shared musical } \\
\text { goals } \\
\text { - Give input and } \\
\text { feedback to fix } \\
\text { problems and evaluate } \\
\text { progress }\end{array}$ & $\begin{array}{l}\text { Steps back and listens to students } \\
\text { self-assessing } \\
\text { - Releases responsibility as tasks } \\
\text { get harder } \\
\text { - Gets important information on } \\
\text { students by listening to them self- } \\
\text { evaluate } \\
\text { - Views collaboration as a life skill }\end{array}$ \\
\hline $\begin{array}{l}\text { May } \\
29 \\
2008\end{array}$ & Marlene & $\begin{array}{l}\text { Student } \\
\text { Freedom }\end{array}$ & $\begin{array}{l}\text { Freedom } \\
\text { - Work in groups on a } \\
\text { project } \\
\text { - Can envision } \\
\text { themselves doing the } \\
\text { task by seeing } \\
\text { classmates as models at } \\
\text { the beginning } \\
\text { - Can move freely, talk, } \\
\text { and make own } \\
\text { decisions in group } \\
\text { - Share results with the } \\
\text { class at the end and all } \\
\text { discuss the task }\end{array}$ & $\begin{array}{l}\text { Relaxed demeanor } \\
\text { - Allows movement and talking } \\
\text { - Creates an authentic musical task } \\
\text { that highly interests students } \\
\text { - Uses student models to think } \\
\text { aloud for the class, instead of } \\
\text { giving strict direction } \\
\text { - Rejects goal of perfect, quiet } \\
\text { class in favor of messier evidence } \\
\text { of learning }\end{array}$ \\
\hline $\begin{array}{l}\text { June } \\
11, \\
2008\end{array}$ & & Wrap-Up & $\begin{array}{l}\text { Large } \\
\text { - Collaboration is ce } \\
\text { - The teacher's role } \\
\text { - What collaboratio }\end{array}$ & $\begin{array}{l}\text { oncepts Discussed } \\
\text { amunity } \\
\text { collaboration } \\
\text { is not }\end{array}$ \\
\hline
\end{tabular}


Our definitions took shape as ways to make the characteristics of collaboration distinct and meaningful for our group. In looking holistically at the emergence of these definitions, I determined that throughout the CTSG, we chunked recurrent characteristics of our definitions together. These essentials of collaboration, extracted from our definitions, became principles. While these principles are derived from our work in the CTSG, they do have applicability beyond these teachers' practice.

Cochran-Smith and Donnell (2006) summarized some thorny issues surrounding making teachers' study public or more widely accessible. They warned that some researchers see the "wisdom of practice" resulting from teacher research as only situated, personal, and relational: that it cannot be a substantial contribution to the body of formal teaching knowledge. While this is not a teacher or action research study, per se, our definitions were socially constructed by a group of three teachers and myself. We used evidence from the participants' classrooms and teaching practice. To prevent the findings in this chapter from seeming too anecdotal or applicable only to the CTSG members, I sought to codify them in three robust principles that would apply to many elementary music settings.

These are principles, not examples, or models. That is a significant difference; principles remain relevant and consistent in their application to a number of teaching settings. Condensing the definitions of collaboration into principles elevates these findings beyond mere anecdotes about collaboration in certain classrooms.

Vignettes and Principles of Collaboration

Our three principles of collaboration are:

1. Collaboration facilitates student self-expression and independence. 
2. Students who are collaborating share goals. The teacher allows space for, or guides students in creating, productive student-student interactions.

3. A teacher collaborating with her students facilitates their movement toward a shared goal. Teacher provides necessary background skills, creates student buy-in for the goal, and then fades away to allow students to take ownership.

The three vignettes in this section exemplify our principles of collaboration. These vignettes feature collaboration as enacted within the elementary classroom: in small groups (four to five students), larger groups (nine), and among a whole class. The varied numbers of students collaborating help to illustrate how our principles might look in several different settings. Within each vignette, I draw from selected literature on collaboration to provide support for our principles.

Vignette one: Karly's class and "Amazing Grace." Karly brought an 11-minute video of her classroom to our first CTSG meeting, which culminated in a class arrangement of "Amazing Grace." The process on video exemplified our principles of collaboration one (collaboration facilitates student self-expression and independence) and three (a teacher collaborating with her students facilitates their movement toward a shared goal. Teacher provides necessary background skills, creates student buy-in for the goal, and then fades away to allow students to take ownership.)

To describe how the nuances of these principles were enacted in Karly's class I will describe the events on video. The narrative is followed by a discussion of principles one and three at work. 
The video began with footage of about nine children making music together. The students on tape were members of the Recorder Club, a lunchtime group made up of some of Karly's highest-achieving second graders. Karly told the CTSG members, "I take the time at lunch to work with my highest and my lowest kids. I spend once a week with the highest ones, and once a week with my strugglers."

She said the class had been working this year on hearing the tonic-dominantsubdominant functions in "Amazing Grace" and improvising over those chord changes. In this video, the Recorder Club students were practicing the song, improvising variations on the tune and bass line. First, about three students danced rhythmically in the background, tapping the steady beat on their bodies, while others sang "Amazing Grace" with Karly at the piano. Next, all nine sang the bass line while Karly played the chords on the piano. The following time through, about four students sang the melody while the others held firm on the bass line.

In ensuing repetitions, children chose which part to sing: bass line or melody. Karly stayed at the piano, and made sure that students were given the opportunity to alter the melodies as well as create rhythmic variations on the bass line. Throughout this rehearsal, students left the piano to choose drums or other non-pitched percussion to accompany themselves. Several students on camera suggested their own twists on the song - usually little tags on the ending of sections — which Karly duplicated in the piano and then taught to the others.

All of these variations were elements of what turned out to be a whole-class arrangement of "Amazing Grace." The Recorder Club ideas for arranging and varying "Amazing Grace" were the seeds of a larger, longer version that was practiced and 
performed by the entire class. Karly's video ended with about five minutes of footage of this whole- class performance of the arrangement. Their arrangement featured student soloists, off-beat clapping, gospel- and rock-style segments, and a student conductor, with Karly playing chords and bass lines on the piano.

In the whole-class version, about six students came forward to sing solos, which were in most cases a variation on the bass line or melody. These variations showed various degrees of rhythmic and harmonic understanding. One student merely sang the bass line with a little rhythmic variation; one student sang the melody with very little change or personalization. Two others added a bit more improvisation as they sang the melody. Two students' improvisations were particularly musical: they showed an awareness of the chord progression as they outlined the tonic and dominant triads within their improvisations.

Karly described the progression on video as a depiction of the method she uses to help her students create an arrangement with her guidance. First, the small group worked with her on solo improvisations and solidifying the bass line singing. In the small lunchtime group, they had freedom to make up other parts vocally or on instruments. With a small number of students, Karly had the freedom to ask them open-ended questions. At one point a girl sat by Karly on the piano bench and sang an eight-bar phrase, performing her own rendition of part of the tune. Karly stopped the piano and asked her, "What inspired this version?" to which the girl replied, "the Elvis recording." The small group atmosphere fostered a non-judgmental atmosphere of creativity and collaboration as the children experimented with ideas that would the next day be 
incorporated within a class arrangement. At the CTSG meeting, Karly explained her take on the Recorder Club events we watched on videotape:

A girl [in the lunchtime club] sang her improvisation on "Amazing Grace." A boy was listening and added a part, so they collaborated together. I asked them for a bass line and got an improvisation, so my question to them was, "How can we bridge this together?" I was at the piano, and they said, "Oh we can do this" and they just went over and got instruments. I never allow them to do that during class, because you can't with a lot of kids around [laughs]. They kind of started putting things together by themselves. (Karly, CTSG meeting one)

At the CTSG meeting we talked about this arrangement sequence after we saw it unfold on videotape. I labeled the Recorder Club members "ringers," or knowledgeable students planted within the group to provide support when the whole class got together.

Karly next described how, when the full class got together, the whole group went along with the basic arrangement of the tune as structured by the small group. "They [the Recorder Club] were ringers in the sense that they had created a lot of that arrangement...but the whole class did agree with the arrangement and were very supportive."

I asked her to say more about how exactly that happened. Jokingly, she said, "it's magic!" The CTSG members laughed, acknowledging the difficulty of getting twentyplus seven-year-olds to agree on creative decisions. However Karly could not explain precisely how the collaborative spirit arose in the creation of this class arrangement. Instead she agreed with our assessment of the difficulty:

I know! It's 'I wanna do my version!' That's what I got a lot. It's really hard for little kids. Even at the second grade level. How can little kids take ownership of this arrangement? I really work on that by giving everyone a chance to be heard. (Karly, CTSG meeting one)

Karly said that the full class agreed on the elements of the arrangement on Monday, April 1, and that on April 3, the day before the CTSG meeting, they had put it 
all together on film. She explained that the arrangement looked and sounded rough on video: "Everything in that video needs to now be fine-tuned. Everything was a little notquite-there...my priority at the time was arranging it, putting it all together." I clarified: "So, the important thing for you was the arranging process, and figuring out how to get input? Not the product. Is that it?" Karly answered by summarizing her belief in the importance of students taking ownership in their own product, rather than the perfection of the final product:

I've done [arranging] before in a small group, going into a big group. Most of them really took ownership... That was five minutes, forty-eight seconds, that arrangement, which I can't believe, for second graders. But it was theirs. I would say $92 \%$ of them really feel like it's theirs. Now my challenge is how can I get everyone really, really into that. To me it was really cool to see that I've had these children for three years now, and they can use their knowledge to create something really meaningful to them. Who cares if it's not perfect? (Karly, CTSG meeting one)

At the end of the video, Karly pointed out how her self-appointed student "choir director" gave the cut-off. With that cut-off as an example, she described her goal of student ownership of work, framing it in terms of gradually minimizing teacher power:

What I'm really trying to do with them is take myself out of it completely, so that, they're having a concert in June... and I will be basically sitting down. So I will be completely out of the equation, it will be completely theirs. So I'm trying to step back slowly. My goal for them is really to be fluent and independent musicians. Obviously they're not independent, at first. But it is theirs. Mostly. [laughs] (Karly, CTSG meeting one)

In this vignette, Karly provides her students with multiple opportunities to acquire the knowledge and skills they need to improvise and put parts together successfully. She does not leave this learning to chance. On the video it is clear that the students have gotten the tools they need in order to enjoy this activity: personalizing the music to make 
a class arrangement. The students seemed secure and confident. At the meeting, she talked about how this learning began back in kindergarten:

Now that I've been there [in Edgeville schools] three years and have seen one class go all the way through, they're a lot more musically fluent. I really try to find strategies starting in kindergarten to have them all love singing and improvising. If they slip through kindergarten without singing, I find they think they're going to keep sliding through! (Karly, CTSG meeting one)

She said she might spend all year in second grade reinforcing the tonic-dominant bass lines of certain songs. Karly told us she tries to create an atmosphere of immersion in the language of music, so students will become fluent. On the video, we saw Karly guiding the students in the independent, creative use of their musical skills. In our meeting, she talked about two facets of that process: risk-taking, and ownership.

When Andrea asked her how she helped more students become comfortable with their own creativity, Karly said her goal is "to help those kids get up, take a risk, by putting them in a safe environment, and by modeling with kids who are willing to [improvise], and remembering them." (Karly, CTSG meeting one). When I asked her what she meant by "remembering them," she said:

The invisible kids, those kids are the scariest to me, the kids that just sit there: "no one's paying attention to me, I'm singing so quietly no one will really care, I'm not causing a problem you know." It's very easy not to pay attention, to forget those kids - they're not causing problems. (Karly, CTSG meeting one).

In this scenario, the CTSG members noticed Karly's commitment to helping each child find an opportunity to express themselves musically. We noted that while the Recorder Club students created some of the patterns that emerged in the whole-class arrangement, the soloists in the arrangement were mostly other students from the class who had not had the small-group tutelage. 
We noticed that having students sing the bass line while others improvised resulted in a shakier rendition than if Karly had played the bass line on the piano for the improvisers. However we identified this as a strategy to keep every child involved and musically "speaking up." Andrea said, "If you're talking about collaboration, that's pretty neat. Some teachers wouldn't have a problem with kids waiting quietly during the solos, but she wants everyone to speak up, to stay musically involved" (Andrea, CTSG meeting one).

Karly also talked about strategies to create a feeling of student ownership. She explained that when students create improvisations, she often writes them down and posts them. "And now they'll say, 'Oh, who wrote that one? Sabrina? Oh Sabrina can we sing your tune? Oh Sabrina we love your tune where you did this!'” (Karly, CTSG meeting one).

After providing students with skills, then guiding them in the creative use of those skills, Karly faded away, by ceding some power to the students in the final product. Karly engaged students in a collaborative effort, but did not seem to fully control the outcome. At the CTSG meeting, we noticed Karly's lack of intervention in the final arrangement; Marlene said, "You can’t even see her half the time!" (Marlene, CTSG meeting one). Dillenbourg (1999) includes "negotiability" (p. 8) as one criterion for defining collaborative interactions. Negotiability is defined in terms of the way it differs from hierarchical situations: "a main difference between collaborative interactions and an hierarchical situation is that one partner will not impose his view on the sole basis of his authority, but will... justify, negotiate, attempt to convince" (Dillenbourg, 1999, p. 9). 
The interaction between Karly and her students, collectively, had a character of negotiation. However negotiation is only necessary when there are real decisions to make. Both she and the students - operating individually, in a small group, and in the whole class - had the ability to contribute to the end product. Suggestions made by students were incorporated. Karly did not dictate who would sing what part, when. In fact, she said that she and the students had only a rough idea of what would happen when they turned on the video camera to record their emerging arrangement project.

Dillenbourg (1999) summarized extant research regarding different ways to set up interactions in which collaboration might reasonably be expected to result in learning.

One important feature of these interactions is the role of monitor or teacher:

This role is often named 'facilitator' instead of 'tutor', because the point is not to provide the right answer or to say which group members is right, but to perform a minimal pedagogical intervention (e.g. provide some hint) in order to redirect the group work in a productive direction or to monitor which members are left out of the interaction. (Dillenbourg, 1999, p. 6).

Acting as monitor, Karly was able to facilitate the second-graders emergent musical ideas as she provided "minimal pedagogical intervention." The intervention she did provide was encouraging but specifically praised students who were good role models. In our meeting, Andrea described Karly's learning environment as she observed it on tape:

An environment in the class that it's okay to make mistakes, you'll get it, you'll develop it, as long as you just keep trying...to use kids as peer role models but not to say, "she's better than you," but to say, "thank you for showing me a great example of a good singing voice" and moving on. (Andrea, CTSG meeting one)

In the small group, Karly listened to the creative musical ideas of the students as they emerged, and then tried to duplicate them on the piano so that the others could hear and remember the phrases. She asked the students for their input and clarified their ideas 
when necessary, by asking, "did you mean this? or this?" We observed on video that Karly spent most of the time on video playing with the students: making music with them from the piano in a gently directive fashion.

Summary. Our first principle of collaboration holds that collaboration facilitates student self-expression and independence. In Karly’s “Amazing Grace” lesson, students began by working together to devise new variations. By creating together, and relating their new snippets of music to others' new ideas, students displayed confidence in a way they probably would not if working in isolation. The freely expressive mood within the lunchtime group of improvisers solidified students' view that they, alongside their friends, can personalize music for their own use.

The sharing, open quality to their creative brainstorming reinforced a valuable lesson: musical ideas can be independently created and then collectively bandied about to make a sum of parts that is amazingly unique. The subsequent dissemination of small group ideas into the larger class-wide arrangement let students trace the journey of their own ideas and creativity from an individual to a collective product. The movement between small- and larger-group collaboration highlights Dillenbourg's (1999) assertion that collaboration is not just for small groups only; a whole class is collaborative when it is peer interactions which are influencing the students' learning processes, and as long as there is a degree of negotiation and synchronous action.

Collaborative principle three states that collaboration is not just among students. Students may collaborate with their teacher, who provides, guides, and fades away. This sort of collaboration occurs when there is space for negotiation, and minimal pedagogical intervention. 
However collaboration between students and teacher, as embodied in this vignette, is not a free-for-all, nor an excuse for lowering musical standards. What made Karly's collaboration with her students successful was the "provide, guide, then fade away" sequence. Karly has worked hard since kindergarten to provide her students with fluency in certain musical activities: awareness of harmonies, improvisation, and being able to sing bass lines as well as melodies to a number of different tunes.

Karly then guided students in using these skills to create a classroom arrangement. A notable feature of this facilitation was Karly's strategy to work with a small group first: guiding nine or so students through the creative process made it easier for her to add another eleven students in the full group later in the week.

Finally, Karly faded away from controlling the performance of their arrangement. She wanted to wean the students from needing her to be a conductor or leader, stating that her long-term goal is to have her students perform without her in concert. In collaborative efforts between classmates, it is easy to imagine an ebb-and-flow as different students take turns assuming more and then less control in a task. While that may have happened among the students in this scenario, more notable was the way Karly's presence faded from view as the students took more initiative in the run-through of the arrangement.

Vignette two: Marlene's class and "Hot Cross Buns." The CTSG's first principle of collaboration states that student self-expression and independence are facilitated by collaboration. When students collaborate they have more opportunities to view themselves as creative individuals who are not dependent on others for modes of expression. 
The CTSG's second principle of collaboration connects student-student collaboration with the presence of shared goals and productive interactions. Teachers can guide these interactions through creating situations in which students are mutually responsible for making progress toward an end, and in which they have the opportunity for positive, reciprocal communication and action with others.

Marlene's "Hot Cross Buns" project, presented at the sixth CTSG meeting, provides vivid examples of the first and second principle of collaboration. Marlene's video began with a small group of students in the front of the room modeling an activity for their classmates. Students were figuring out the notes and the notation to a known song, "Hot Cross Buns" and sharing in the responsibility of writing the notation on a large piece of paper to be shown to the class.

Karly said that Marlene immediately had the students' attention because she was using a group to in the front of the room, fish-bowl style, to model the task. She said, "it was almost like a think-aloud for the class to see." Using students as a model immediately set the activity up as something students could do, and illustrated to the class how a positive group dynamic might look. This is an example of a teacher guiding productive interactions.

In Marlene's video, we noticed that all students seemed completely invested in the project. An outward physical manifestation of the group's shared motivation was the fact that that students were up and out of their seats, leaning over each other to talk about what was being written, singing the correct pitches into one another's ears, helping and even arguing. I said:

It's a real positive that Marlene did not make them sit in their seats. That completely changed the whole tenor of the lesson. If I'm thinking about 
collaboration, I'm also thinking about teachers who might get antsy if kids are up and moving around, who might say, "you're going to work in a group, but don't move around the table. Pass the paper around instead."...That movement and positioning: really important. (Ann Marie, CTSG meeting six)

Allowing students the space to organize their own interactions, figuratively and literally, seemed to result here in productive interactions. But we wondered how Marlene had been able to instill such a sense of pride and caring about the work, and where that motivation came from. I asked, "Why were they standing up and watching? Why were they monitoring? Why was it that they cared? That it was meticulous? That sort of "buy in," that we keep calling it, I really felt it here." Karly said this keen interest in and observation of the group efforts manifested itself in meticulous work: "Did you see how much they cared? How much they helped each other so it could be right? And it had great meaning for them."

As we worked through our analysis of Marlene's video, Karly and I came to two conclusions. One was that the collaboration we saw here was a result of students having a clear goal in mind, that of being able to present their work to the class. The other was that the ability of the students to talk and physically move around the room was crucial to the their collaboration.

Karly said, "They were positioning themselves to see the music, they were collaborating, there was absolute buy-in, they saw a need and they got themselves over there to work." She said seeing their classmates model the task at the beginning made students aware that this was an authentic task, not busywork: "it promoted contextual learning...they weren't saying, 'I'm the B person, I'm going to put my note down and not look at anything else today. That was huge for this class." 
Marlene agreed, saying that she now realized that giving students opportunities that varied the amount and types of interactions they had seemed to result in more cooperation and deeper learning:

I learned that I don't want them to do the same thing, always. We've been playing recorder for a few weeks now and I don't want to just sit down, play, and watch me. Different things, different ideas, working in different groups, is better. (Marlene, CTSG meeting six).

Summary. I see the first collaborative principle at work in this vignette, particularly in terms of the way the collaboration generated independence. Students were encouraged to come to their own conclusions about the notation, seeking input from fellow students but were not closely monitored by the teacher to ensure the right answers. Rogoff (1990) states that children strengthen understanding of concepts when they are given the freedom to consult with others, independently of the teacher. She says, as students "collaborate and argue with others, they consider new alternatives and recast their ideas to communicate or to convince" (p. 195).

Students were given a task that allowed them to regulate their own participation in the group and to have plenty of opportunities to convince or be convinced. There are a number of ways Marlene could have taught her students the notation for "Hot Cross Buns," including just passing out the sheet music. However, she created an activity in which students had to derive the notation themselves, trusting that the act of collaborating with others would take her students the first step down the path of being independent music-readers. The collaboration facilitated student independence as students had to 
come up with their own ideas in autonomous groups, and then present their product to the whole class in a convincing way.

Dillenbourg (1999) states that for a learning situation to be collaborative, peers should be at approximately the same level, having symmetry of action, knowledge, and status (p. 7). If students have the same range of actions available to them, similar opportunities to contribute to a project, and feel a sense of equality within the standings of members of the group, students are likely to have productive interactions. Our second principle goes a step further. Not only do teachers have the ability to allow students the opportunity to participate on an equal level, but they can take steps to guide and promote these interactions.

Unpacking this vignette, I find that Marlene guided her students in productive interactions in two ways. First, she allowed them space to move, talk, and share ideas. Marlene stated in several contexts throughout this study that she was often concerned that students up out of their seats and talking would somehow indicate a lack of learning, and reflect poorly on her. But in this situation, less control over their talking and movements actually ended up paying dividends in the students' ability to construct their own productive interactions.

Second, Marlene was able to equalize the status and symmetry of action of students in the class. By using student models to demonstrate the task, she made it clear that this was something everyone could do, and in which everyone could have a role. The task had enough components that everyone was important and needed. She also built in a shared goal by telling the class that they could share their written product at the end, and then decide what was good about each group's endeavor. She guided positive 
interactions by motivating students to assist one another in the groups not for the competitive result of coming up with the right answer, but instead, to do their best work and share with their classmates a representation of their best thinking.

Vignette three: Andrea's class and "Sally Go 'Round the Sun." The way Andrea led her class through progressively more difficult iterations of a performance of a round with accompanying movements is an example of collaborative principle three. Much like Karly did with "Amazing Grace," Andrea provided her students with the knowledge of the song and movements, guided them in practicing the round with fewer and fewer students on a part, and then faded away as students began to fully take charge of their performance.

However, the differences between Karly's lesson and Andrea's lesson illustrates two very different enactments of our second collaborative principle. In the "Amazing Grace" vignette, Karly provided minimal pedagogical intervention throughout. In contrast, Andrea monitored and structured every step of the "Sally Go Round the Sun" lesson. The experience was still collaborative for the students, however, because of two main elements Andrea built into the following sequence: providing information, then creating student buy-in toward the goal, followed by student taking full ownership.

First, during the "provide" portion of the sequence, Andrea gave numerous suggestions for how to create musical ensemble. Calling them "tiny things," she gave very specific strategies students could draw upon. However, she did not tell the students to do these things.

For example, Andrea said, "If the circle is getting smaller, you can take a step to the right to help everyone make it bigger again." She did not say, "Class, the circle is 
getting too small. Everyone take a step to the right to make it bigger." She suggested, "When I am singing a round, I like to sing softly so I can hear the other parts along with mine." She did not say, "Sing softer and listen to the other groups' part."

Andrea provided the students with the information they would need to make the ensemble better, but left it up to them when to use it.

Second, during the "student buy-in" portion, Andrea elicited student input through use of open-ended questioning techniques. Andrea was guiding the students in figuring out how to make the performance more solid with fewer on each part. She put the onus on them to identify what was wrong and how it was affecting their ability to have a clean, tight performance with a perfect ending. She repeatedly asked students questions like, "How can we make this better?" and "What do you think happened here?" She would then take their suggestions, reword them to make sure she had it correct, and help the students incorporate them into the next run-through.

For example, students suggested stepping louder to hear the steady beat in their feet as they sang, thinking that would help them align the parts of the round. Andrea said, "So you're saying that you are having trouble feeling and hearing the steady beat when we're singing? Okay let's try it with a beat." After several times through the round, the stepping had become counterproductive stomping. Instead of telling the class to stop stomping, Andrea wisely chose a particular student—who had been visibly disturbed by the stomping noise - to make the next suggestion. When he said the class should tone down the stomping and even try thinking the round, another child chimed in, "Now I have to put it [the beat] in myself!" The round was much more secure after that. 
Summary. Andrea's language throughout, especially her use of "we," suggested shared goals; she made remarks such as, "If we're singing the song in our head, we'll have success" and "as we're going along, we're getting more together, but our ending was not. Now can we work on our ending?" The goal of a great ensemble performance was gradually transferred from being Andrea's lesson objective to being the students' desire. This transfer is an example of collaborative principle three, as Andrea gradually moved the students away from needing her as a director, and put the onus on them to stay together. This happened in a sequential way.

Karly said:

By the end I felt like there were less sheep and more leaders. But by that I mean they were each taking responsibility. They were listening to each other and trying to blend their own voices, not just going mindlessly along with the crowd. They seemed to think, "We have to be really responsible here now that it's getting really hard." (Karly, CTSG meeting five).

Collaborative principle one was also made visible in this lesson. When describing her goal for the lesson, Andrea alluded to musical independence: "I wanted to see if they could not rely on my leading the singing, if they could really figure it out for themselves and have it internalized" (Andrea, CTSG meeting five). Andrea used a collaborative atmosphere to help generate musical independence; through the students' working together, making suggestions, and interacting they were able to wean themselves away from her direction and stand on their own to perform a round with a perfect ending. Connection of Principles to Past Research

To verify the sturdiness of our principles, I went back into the literature and, with our CTSG principles of collaboration in mind, read several studies documenting student collaboration. I found that our principles apply to situations other researchers have 
deemed collaborative, and corroborate some theories of collaborations previous researchers espoused.

Roschelle (1992) found that students may jointly change their concept of something — in his study, certain laws of physics — as they collaborate. The process of changing, and agreeing on the changed conceptual understanding, is convergence. One of the ways in which convergence happens is when students talk in ways that allow them to explain their own knowledge, understand others' ideas, and fix any misunderstandings or discrepancies.

This aligns with our principle two of collaboration. In the CTSG, we found that in order for students to converge on a new meaning, in other words, learn something about music, they need to be able to talk. Teachers need to allow space for this talk to happen, and provide mechanisms and situations for the talk to be productive.

Roschelle and Teasley (1995) studied a pair of students solving physics problems. While in general the pair was productive, and generated new knowledge, the degree of collaboration within their interactions ranged from high to almost non-existent at one point. The lesson from that study which I am apply here regards the elusive quality of collaboration. The researchers state that for collaboration to happen, "individuals must make a conscious, continued effort to coordinate their language and activity with respect to shared knowledge" (p. 94).

Principles two and three of collaboration relate to shared goals: goals shared among students, or between teacher and students, or both. In the CTSG videos, the situations which we deemed productive, effective for student learning, and collaborative featured a visible, tangible effort to share goals. But for goals to truly be held in common, 
everyone must accept them. The CTSG teachers portrayed a number of ways to instigate group ownership of goals, and regardless of the teaching or learning situation, it is clear that collaboration required a great deal of Roschelle and Teasley's "conscious, continued effort." Therefore, merely working alongside one another is not enough, the target must be shared by all, which is why principles two and three specify "shared goals" along with space or teacher facilitation to create and maintain them.

The CTSG members consistently defined collaboration as a phenomenon that is not an end to itself, but that starts something bigger and more worthwhile. For example, in our evolving definition of collaboration throughout the CTSG we credited collaboration with enabling personalization of musical choice, freedom from reliance on the teacher, creativity, and ownership of new ideas, among other things. I found that summing up the power of collaboration in collaborative principle one was helpful, as all our ideas could be encompassed within an umbrella statement: collaboration facilitates student musical self-expression and independence.

This principle one dovetails with Wiggins' (2000) findings in a longitudinal study of classroom collaboration, which combined findings from eight years of studies and additional data from her elementary classroom. She found that successful classroom musical collaboration results in a process that "generates within the individual a sense of possibilities" (p. 49). As students shared ideas and reshaped them in response to interactions with others, they became confident about their own creations and were more likely to change and play with musical ideas in response to others' input.

Wiggins also searched her data for positive and negative evidence of shared understanding, or "a group's vision of a work in progress" (p. 85). She concluded that 
when the vision was shared clearly, interactions were collaborative and resulted in successful work. This finding also connects with principles two and three of collaboration, as the CTSG teachers' videos demonstrate that true collaboration requires students to share a vision or desired outcome with each other and/or with the teacher. Principle three describes a sort of collaboration involving a sequence in which a teacher facilitates her students' progress toward a shared goal. Younker and Burnard (2004) found a similar pattern in the teachers' interventions within groups of students collaborating to compose and arrange. They found that teachers taught by modeling, and then guided students in a learning process by asking them to describe, clarify, and reflect. The researchers state that the role of teacher as facilitator could be refined in further research on student collaboration.

We talked often in the CTSG about the role of teacher as facilitator. Our definitions of collaboration included much language about how teachers enable, allow, reinforce, and provide space for collaboration. Principle three summarizes our multifaceted views of the teacher's role into a shorter and more direct idea: one way collaboration happens is when teachers facilitate it through a process.

In Barron's (2000) study of sixth-grade small group collaboration, she suggests that a worthy goal for education is to develop teachers' ability to allow their students more opportunities for productive collaboration. I had this study in the back of my mind as I began this project. I assumed that teachers would characterize collaboration in elementary music as difficult to engineer, because student interactions are apt to turn problematic, and student input is too unpredictable to be accorded extensive class time. I 
thought the CTSG would develop Marlene's, Andrea's and Karly's abilities in finding opportunities for their students to collaborate.

The CTSG did work as I thought it might. The teachers learned about collaboration from one another; each participant cites greater comfort with and competence in facilitating collaboration in their classrooms. I believe they are all finding more opportunities for their students to collaborate.

The teachers' acquisition of increasingly refined knowledge of how to facilitate collaboration is important. However, the analysis of the extant aspects of collaboration in their classrooms is probably just as important. The dissection and analysis of the videos both at the meetings, and in my subsequent examination of the data, illuminate a number of ideas and suggestions that the CTSG members were already incorporating in their practice. Taking apart the lessons on video helped us better understand and name some of these excellent strategies. This paper would be incomplete without a complete listing of all the practical considerations and ideas for the collaborative elementary music classroom. They are included in Chapter Seven, under implications for practice. 


\section{Chapter 7}

\section{A CONTINUATION}

The story of the CTSG is a continuing one. In summarizing the findings from this project, I am aware that our look at the many aspects of collaboration was fulfilling and illuminating, but still too brief. What we learned about this collaborative teacher study group and collaboration in elementary music has implications for future practice in both areas, but there is still much more to learn. I have more questions, plans for continued projects with these same three teachers, and ideas for future CTSG's. I have suggestions for future research on collaboration that stem from my continued curiosity.

In this chapter I summarize the design of the study and the findings. Next I present implications for practice in teacher collaboration and professional development, followed by implications for practice in elementary music student collaboration. I make suggestions for continued research in teacher and student collaboration. Finally, in the form of a personal letter, I bring our attention back to the women who continue daily to work for collaboration and community: Marlene, Andrea, and Karly. Summary of the Study

The purpose of this study was to examine the experiences of three elementary music teachers and a researcher involved in a collaborative teacher study group (CTSG) designed specifically to focus on student collaboration in elementary music classrooms. The research questions were: 
1. How do the participants describe their experience in the CTSG?

2. How has the focus on collaboration in the CTSG changed the teaching practice of these teachers?

3. What can these music teachers tell other music educators about collaboration?

Definition of a Collaborative Teacher Study Group (CTSG), Participants, and Site

The collaborative teacher study group (CTSG) was a group of three elementary school music educators and me. We met together regularly to support each other in two goals: the participants' own professional development, and greater knowledge about collaboration in the elementary music classroom.

The three elementary music teacher participants in this study have public school teaching jobs. Marlene teaches at three elementary schools in an urban school district, Ridgeland, in upstate New York. In 2007-2008, she was a first-year teacher in Ridgeland. She has twelve years of teaching experience. Andrea is a teacher in one school in the suburban district of Pleasantford, where she teaches music to grades one through five. Karly taught kindergarten, first, and second grade general music in one elementary school in a suburban school district, Edgeview, next to an urban school district in upstate New York. Andrea and Karly are both third year teachers. I am the researcher, and also a member of the CTSG. My actions and perceptions as collaborative teacher study group facilitator are part of the study.

The CTSG met seven times after school on Thursdays, for two hours. Our work was structured according to my adaptation of the protocols from The facilitator's book of 
questions: Tools for looking together at student and teacher work (Allen \& Blythe, 2003). At each meeting one teacher presented a 10- to 12-minute video from her classroom which we would watch and discuss. We took turns hosting the meetings in our homes and leading the discussion according to the protocol.

Related Literature in Teacher Community

Site-based or local teacher work groups, inquiry groups, and communities of practice are increasingly used as models for teacher professional development: models that provide a place for shared, reflective inquiry around problems and issues of teaching.

The literature on teacher professional development communities—inside and outside of music — defines a number of characteristics that seem to contribute to teacher learning. These are (a) the length of commitment to the community; (b) the tension between the goals of improving content area knowledge and pedagogical skills; (c) the way teachers with varied goals for development participated, and in different roles; (d) the avoidance of or enabling of honest examination of teaching practice; and (e) the ways teachers brought issues forward for discussion. Selected studies illustrate these five characteristics of teacher community to different degrees, and provided a basis for this study (Dolloff, 1996; Grossman, Wineburg, \& Woolworth, 2000; Horn, 2005; Junda, 1994; Little, 2003; Robbins, 1995; Roulston, Legette, DeLoach, Buckhalter-Pittman, Cory, \& Grenier, 2005; Rousseau, 2004).

\section{Collaboration in Education}

Within educational research in the last half-century, collaboration has been examined to better understand the processes of group interaction and how they contribute to student learning. Researchers have looked at variables such as children's expertise, 
developmental level, style of interaction, social skills, type of interactive tasks, and instruction in collaboration. A number of studies of student collaboration and the factors that affect the success of collaborating groups framed my work in this study (e.g. Azmitia \& Perlmutter, 1989; Barron, 2000; Burnard, 2002; Chinn, O’Donnell, \& Jinks, 2000; DeLorenzo, 1989; Dillenbourg, 1999; Dillon, 2003; Fischer \& Mandl, 2005; Palincsar, 1998; Palincsar \& Herrenkohl, 2002; Perret-Clermont, Perret, \& Bell, 1991; Roschelle, 1992; Roschelle \& Teasley, 1995; van Boxtel, van der Linden \& Kanselaar, 2000; St. John, 2006; Wiggins, 2000; Younker \& Burnard, 2004, 2008).

\section{Definition of Collaboration}

There is a lack of consensus in the literature about what constitutes collaboration.

I use Roschelle and Teasley's (1995) definition:

Collaboration is a process by which individuals negotiate and share meanings relevant to the problem-solving task at hand...Collaboration is a coordinated, synchronous activity that is the result of a continued attempt to construct and maintain a shared conception. (p. 70)

I also draw from the work of Dillenbourg (1999) to explain cooperation versus collaboration, symmetry, division of labor, size of collaborating group, negotiation, and the role of the teacher in the context of this study.

\section{Methodology}

This study is a social constructivist inquiry, meaning I was primarily interested in the beliefs and viewpoints of the participants as they were socially constructed in the CTSG. The CTSG is an environment of collaboration which I tried to understand from the inside out. I used evidence from our meetings and interviews to trace the changes that occurred over time in the CTSG participants' individual and collective viewpoints and perceptions, regarding teacher learning, professional development, and collaboration 
between teachers and between students. I used their words to tell each participant's story and to find the commonalities and differences between their experiences. I talked with the participants about my interpretations of what was learned in the CTSG meetings: we ended with a shared understanding about collaboration in elementary school music developed throughout the course of the study.

\section{Sources of Data}

Semi-structured interviews. I had 90-minute interviews with each of the participants before and during the course of the CTSG, during the weeks of March 24 and May 6, 2008.

The first interview was designed to find out as much as possible about what Karly, Marlene, and Andrea think about professional development and collaboration. The second interview in this study, conducted after the fourth meeting, allowed Karly, Marlene, and Andrea to reconstruct details of their participation in the CTSG, and tell about any effect on their teaching practice or student achievement. All interviews were transcribed verbatim.

Videotape of CTSG meetings. Digital videotape was used to record the events of each meeting of the collaborative teacher study group. All seven meetings were transcribed verbatim.

Artifacts from CTSG meetings. I used the notes we wrote on the protocols to confirm words or statements as I coded the data from the interviews and transcripts. Personal emails from participants: these provided new information, used to add to or triangulate findings. 
Participant-researcher log. I kept a log detailing the events of the CTSG meetings, including communication I had with the participants, meeting protocols and agendas, and my meeting notes. I did not code this $\log$ in the analysis procedure; rather I used it to confirm timeline and location details. Analysis

As I watched the video and read the transcripts, I reflected on the research questions. I searched their words for data to help me recount each person's particular narrative. I looked for information that would let me appreciate how Marlene, Andrea, and Karly understood their world as teachers and learners. I wanted to see if individual processes of knowledge generation could be traced in their words, and interpret what, if any, changes occurred as a function of belonging to the CTSG. Because this was a social constructivist inquiry, I was also interested in what the collective convergence was around our meanings of collaboration.

Summary of Findings

Research questions one and two, by participant. Research questions one and two are closely entwined. In searching the data to answer how the participants describe their experience in the CTSG, I also found evidence that the focus on collaboration in the CTSG changed the teaching practice of these teachers. Often the participants talked about their experience in the CTSG in terms of its impact on teaching practice. The findings regarding the members' CTSG experiences are presented below for each participant.

For Marlene, the experience of being in CTSG boosted her confidence in her professional knowledge and gave her an opportunity to share her considerable expertise with others. At the beginning of the CTSG, she questioned her ability to connect with 
students and wondered if her organization of the learning environment was effective. At the end, she spoke about her own professional knowledge in a more confident way. Marlene said the CTSG had immediate relevance to teaching practice. The CTSG members helped her identify strategies for particular situations as well as identify episodes of her own teaching that, when viewed through the lens of how they facilitated student collaboration, turned out to be outstanding examples.

Marlene credited the CTSG format for not only changes she made in activities she did with her students, but for changes in the ways she saw her students. She said seeing her students on video, through the eyes of her fellow CTSG teachers, had made her realize that not everyone needed to appear as if they were learning in the same way. Categorizing the ways collaboration might look in the classroom gave Marlene other vocabulary to analyze and describe what her students were doing.

Andrea experienced the CTSG as a powerful encounter with reflective teaching. Seeing and discussing her classes on tape led Andrea to a more habitual reflective examination of her teaching and the learning environment she helps to create. She was able to think less about her teaching, and more about learning, in an increasingly analytical way. The CTSG experience also helped Andrea develop an expanded awareness of student collaboration, and an expanded awareness of her own role in facilitating student collaboration. She paid new attention to the different ways collaboration might look in the classroom.

The experience of the CTSG profoundly affected Karly's outlook on teacher collaboration. She came into the group isolated and frustrated with what passed for serious discussion of music education in other groups of teachers. In contrast she said the 
CTSG was a wonderful way to approach learning, which made her want to become a better teacher. She was grateful for the significant conversations about teaching we had. Karly absorbed elements of the other CTSG members' practice within her own, and made explicit what she had learned from them. Her views of collaboration expanded; she talked about sequencing, promoting community, and awareness of student input in direct instruction. The quality and depth of her reflection on these changes makes me believe the CTSG experience had a substantial effect on Karly's teaching practice.

Research question one, across all participants. After answering research questions one and two in terms of each participant, I identified two broad themes that recurred across all of their experiences. The extent of findings within these two themes for me substantiated the necessity of further exploring research question one in its own chapter.

The first theme is how the CTSG was professional development that supported teacher learning and change in practice for these teachers. I further divided this theme into a discussion of six elements of the CTSG that positively or negatively affected its capacity as an effective professional development experience: (a) the collectively generated knowledge in the CTSG; (b) the lack of development of teachers' musical content knowledge; (c) the discussion of students' musical content knowledge; (d) the necessity of video; (e) the importance of the protocol in effecting change in teachers' analysis of practice; and (f) defining and observing student work, versus teacher work.

The second broad theme in common is how the CTSG was a form of professional development that partially combated isolation for these teachers. The CTSG members had a sense of not being full-fledged members of their school communities. Connections 
within the music education community were no stronger. The participants felt cut off from what other music teachers do. Our meetings energized the teachers, gave them new ideas appropriate for their context, and increased their feelings of belonging to a professional, intellectual community. However, the CTSG did not substitute for the teachers' desire to have on-going conversations with their school and district colleagues.

Research question three. The third research question I asked was what can these teachers tell other music educators about collaboration? The first thing the CTSG can tell other music educators is how we defined collaboration in elementary school music, which we socially constructed over the course of our meetings. From these definitions I have extracted our three principles of collaboration; these principles are the second part of what the CTSG teachers can tell other music educators. Our principles of collaboration are:

1. Collaboration facilitates student self-expression and independence.

2. Students who are collaborating share goals. The teacher allows space for, or guides students in creating, productive student-student interactions.

3. A teacher collaborating with her students facilitates their movement toward a shared goal. Teacher provides necessary background skills, creates student buy-in for the goal, and then fades away to allow students to take ownership. Implications for Practice in Teacher Collaboration and Professional Development

This study contributes to our understanding of one form of music teacher professional development: a collaborative teacher study group that generated teacher learning. In the CTSG, Marlene, Andrea, and Karly learned more about themselves, their teaching practice, and their students. The literature on teacher community has 
consistently reported that teacher talk and conversation with colleagues is invaluable for teacher learning; this study joins others in providing a rationale for including mechanisms for collaboration in teacher professional development.

I have summarized selected research on professional development in teacher community (Dolloff, 1996; Grossman, Wineburg, \& Woolworth, 2000; Horn, 2005; Junda, 1994; Little, 2003; Robbins, 1995; Roulston, Legette, DeLoach, BuckhalterPittman, Cory, \& Grenier, 2005; Rousseau, 2004) in terms of four specific actions within communities that foster more productive talk and result in the growth of teacher knowledge. These actions are (a) having ways to make teaching practice visible to colleagues for realistic discussion, (b) being able to participate within the community by taking on various roles, (c) honoring the strengths and expertise of each member in the community, and (d) providing ways to have honest, sustained talk that gets to real issues of teaching and learning. I will address the implications for practice inherent in each of these in terms of findings from this study, and ask several related questions for each.

Making teaching practice visible. It is clear the CTSG members benefited from watching one another's classrooms on video. Sessions where other teachers or clinicians simply talked about teaching left them wondering skeptically about what really went on in other locales, and how to translate ideas into their own context. To see teaching practice on film eliminated doubt; the CTSG teachers know how these strategies work in real-life situations. They studied how strategies were applied in different settings.

In music teaching there are very strong norms of privacy and autonomy; norms inadvertently reinforced by the literal and figurative isolation many music teachers face. I recommend that professional development experiences find a way to transcend these 
tendencies. Inviting others into the classroom, either in real time or through video, may be scary and threatening. But seeing real teachers instructing real students is the only basis for pragmatic, rational conversations about music education. Any other discussion becomes vaguely fictionalized; teaching practice is almost always made fuzzy by the language of its retelling.

What further benefits might accrue to teachers allowed to actually visit one another's classrooms on a regular basis? I believe teachers will be eventually relieved and reassured by breaking down the strong barrier of privacy and allowing others in. At a recent workshop I conducted, a young teacher told me afterward she feels like "a fraud" because others assume she is doing brilliantly in the classroom, by virtue of her resume. However, alone with the students, she sometimes struggles. She said she has told others about her problems but the quick fixes suggested by others did not work, which further compounded her feelings of inadequacy. If sharing of video or real-time visits were a regular feature in a professional community for this teacher, she would understand that not only do the same problems she faces recur everywhere, they have been dealt with in many effective ways. She might feel relief from not having to hide her uncertainty.

However, I stress that this sort of relief can only be obtained through the use of video or visits; merely getting together in collegial groups to talk about realities of teaching will not have the same impact. Only by seeing what actually goes on in music classes will we strengthen our basis of professional knowledge on which to build improvements in teaching and learning for all. I hope that supervisors and leaders in our profession will see the necessity for looking into music classrooms in ways that are not onerous, not connected to formal evaluations, but rather, are intended to develop and 
enhance teaching practice in a way only possible through transparency and openness. The only other alternative is to not share and to not talk. This alternative sustains a view of music education as a series of individual programs, built by individual teachers, which range from unsuccessful to unremarkable to outstanding; the reasons for their success or failure are often only theoretical, rumored, or legendary. We often do not know why a teacher is said to have an outstanding program, because usually we see only the product - the concerts, the musicals, the graduates - and never glimpse the daily progress in the classroom. I believe transparency and openness across schools and programs would help build our understanding of music teaching and learning for all.

Taking on various roles in the community. Members of the CTSG were able to take on varied roles within the group: host, facilitator, presenter, note-taker. However members of the CTSG were also able to take less-formalized roles as well: suggestiongiver, idea-needer, supporter, cheerleader, vulnerable teacher, authority, novice. The ways in which we were all able to participate shifted throughout depending on the topic under discussion or the mood of the participants. These constant shifts equalized the power within the group and created a feeling that no one had to be "on" all the time. I also believe that the collaborative, democratic, symmetrical nature of our meetings helped each of us invest in the process; we all seemed to feel ownership of our group and its collective bank of ideas.

In future practice, I recommend that while a CTSG needs an administrator to be the clearinghouse for messages, scheduling, and emails, a permanent facilitator is not necessary. I could have easily been replaced in this group by another elementary music teacher, as I shifted between roles just as fluidly as everyone else. They did not need me 
to stay in place as facilitator; these teachers were fully capable of reading the protocol and running the meeting themselves. In fact, I wonder if the group would have been more effective with a fourth video-sharing member. I never brought video myself because I am no longer in the elementary school classroom; perhaps even that distinction solidified my role as along a different track than the other three. It is most advantageous for all participants to take turns in all roles.

My next question for future practice regards the identities the teachers come in with, and how those related to our ability to participate along multiple roles. I am a university general music methods professor; they were elementary music teachers. While we took on different roles within the group, we did not have to stretch or change our identities at all to be able to fully participate in discussions about elementary music.

However, I wonder if this limited our perspective. Had I brought video from, say, my elementary general music methods course, I believe I could have learned valuable things about my own teaching and my university students. I think the CTSG teachers would have been able to analyze my video for aspects of collaboration. The stretching necessary to apply concepts across age levels might have actually facilitated our thinking about transfer. We might have drawn more connections between the nature of our own collaboration in the CTSG and the students' collaboration; an extension of thought which did not occur in the CTSG despite my idea that it might. We could have in a sense practiced applying collaborative concepts in other ages and settings.

Also, had there been an elementary classroom teacher in the group, she might have provided new insight into what the students were doing on video. She might have noticed something we missed because of our shared, but perhaps myopic, music teacher 
viewpoint. Also, this teacher might have changed our discussions about how classroom teachers use music as break time and never seem to want to meaningfully integrate music. I do not know if these feelings would have been glossed over in the presence of a classroom teacher, or if she would have been able to explain the classroom teacher viewpoint. She might have been able to make suggestions about how to better connect with classroom teachers, or perhaps think of ways classroom teachers might better support music teachers.

In future CTSGs, it would be interesting to have two participants from each school represented: a music teacher and a classroom teacher. I believe this would enable us to stretch our minds to participate beyond our music teacher roles. We would need to look at teaching and learning even more broadly. However the presence of enough music teachers would also provide the nitty-gritty specificity that Karly, for example, always wanted. The participation of an additional staff member from a school would also help to answer the dilemma posed by recent research in teacher professional development (e.g. Penuel, Fishman, Yamaguchi, and Gallagher, 2007): the amount of institutional support in place for teachers can drastically affect their ability to make meaningful change. Having another member participating from their site might help teachers have conversations throughout the week and share reflection on the CTSG informally throughout. Two members from the same site would also be a small gesture toward institutional support; while not a full-fledged, school-wide implementation it would still be helpful to have two people on staff considering the same issues.

Also, a CTSG might be very effective with the inclusion of music teachers from other grade levels. While the CTSG teachers said they appreciated professional 
development that targeted their own specialty, I think a vertically aligned CTSG would be powerful in terms of strengthening the intradistrict communication and curriculum organization so often found lacking by these three teachers. If, for example, an elementary general music teacher was in a CTSG with beginning, middle, and high school band teachers in her district, the discussion might prove to be different than other music department meetings. The CTSG focus on structured, video-based discussion might clarify the similarities and differences in music education P-12 and provide the honest examination of student learning that might work toward bridging gaps between grade level curricula.

Honoring the strengths and expertise of each member in the community. As we participated along various roles in the CTSG, we all had the chance to offer our individual expertise. Teacher community professional development seems to be richest and most effective when it honors the expertise already in the room. This is not to diminish the important role that can be played by someone who is an authority or veteran. Rather, locating the expertise and collective wisdom of the teachers who are participating in professional development and finding ways to bring it forward also taps a rich resource. The knowledge teachers bring of context, practice, and better and worse ways to do things in a classroom cannot be underestimated. A CTSG offers a means for teachers to talk through things that work well for them, and in the process, engage in reflective discussion with colleagues about why they work.

This model of professional development flies in the face of many professional development structures that are based on experts presenting information to participants. Even though in many of these cases the expert is herself a practicing teacher, the format 
itself is not conducive to reflective examination of teaching practice. The effect of teachers talking together to unpack teaching is profound. Things that seem to work well can be named, defined, and closely examined for their real efficacy in facilitating learning. Sometimes techniques that seem to already work well can be changed or improved, upon close analysis of their role and function. A teacher who, in conjunction with skilled colleagues, is able to identify and recognize the specific area and depth of her expertise or many talents, and may in turn be more confident and more apt to sustain further learning and sharing in these areas.

Again, I stress: professional development that will be effective in this regard is not as simple as teachers casually sharing ideas in a circle. As the above points indicate, sharing expertise is far more involved than that. It also requires ways to make teaching practice visible to colleagues for realistic discussion, and being able to participate within the community in various roles.

Ways to have honest, sustained talk. Our conversations in the CTSG were productive, interesting, and far from superficial. I believe this is because we used a discussion protocol. The protocol ordered our talks and let us know what was coming up. The protocol provided some reassurance the conversations would not turn ugly or contentious, as the facilitator can direct the meetings through the protocol and guide the opening of topics and uncovering of new ideas. The protocol we used kept us remarkably objective and yet helped us find many "a-ha!" moments. There are no moments in the collaborative consultancy protocol that call for group members to share whatever they think or feel; the protocol calls for describing, analyzing, and speculating on evidence 
from the video tape. It is possible that teachers could pipe up with unwanted, uncalled for opinions or criticism, but in my view the protocol makes that prospect unlikely.

However, as mentioned in Chapter Five, while I credit the protocol for the productivity of our conversations, it was not the only element of our group that contributed. I attribute some of our success to the natural sensitivity of Marlene, Andrea, and Karly; however, it is hard for me to imagine how that happened as I gave almost no thought to our group chemistry before we began. I needed insightful, dedicated, and willing elementary teachers and they were eager to join. Perhaps the very fact that they were willing to join a "collaborative teacher study group" belies something about their personalities: they were willing to give hours out of their busy lives to collaborate with others to study teaching, after all. If a group of teachers was identified by an administrator and told to participate in a CTSG for professional development, I wonder if the tone of the conversation would change. I wonder how involuntary participation in a CTSG would affect the formation of community. I also question what would happen in a CTSG whose members have a history of disagreement.

The topic of the CTSG may also have contributed to its success. We had no history of disagreement around the idea of "elementary school musical collaboration;" however, we potentially might have had disagreement around issues of methodology. The three teachers had taken summer courses in Music Learning Theory, Orff-Schulwerk, and Kodàly; understandably, they are devoted to putting those ideas into practice. I do not know if this group was successful because of our lack of preconceived notions about collaboration. 
In the CTSG, compared with other groups of music teachers I have visited, there was a remarkable lack of automatic reactions to certain words such as audiation, improvisation, creativity, and musical literacy. I attribute this lack not only to the broad, heretofore undefined topic of collaboration, but to the fact we talked about what we saw and heard on tape and had mechanisms in place for ordered discourse based on realities of music teaching and learning. If a knee-jerk reaction did occur, we were able to go back and look at the video and talk about what we had seen to determine if our assumptions were accurate in this specific instance. I believe that the structure of the CTSG would stand up to the pressure of analyzing and unpacking even a more loaded subject, and I would like to try to form a CTSG around another, more defined topic to compare the results.

I stand firmly behind this belief, rooted in previous literature and confirmed by this study: if the four elements discussed in this section are present in any CTSG, the chances are high that it will work to support teacher learning and change in practice. The alternative is not to try at all, but I believe the potential value of a CTSG makes it worth trying as professional development even if there are possibly detracting factors.

\section{Implications for Practice in Student Musical Collaboration}

The CTSG was intended to provide support for teachers seeking to give opportunities for their students to collaborate. The research has repeatedly said that students need to be guided in productive collaboration, and that teachers may need to be guided in how to do so. This study provides valuable information about what elementary music teachers think about music student collaboration, and how to facilitate or include it 
in their classrooms. We discussed collaboration in a variety of musical activities from grades kindergarten through six, a wide viewpoint missing from previous literature.

The teachers in this study brought forward information about how collaboration occurred in their music classroom. The collective thinking of the group helps contribute to the body of knowledge about what collaboration looks like in the music classroom, and how its use may be enhanced. Some of these considerations were accepted, usual practice for the CTSG teachers; some were new ideas. Some of our thoughts about collaboration are practical tips, and others are more reflective. Tacit knowledge about teaching in a collaborative music classroom emerged; in some cases we were able to explain and name this knowledge in order to make it clearer for us and for others. It is beyond the scope of this paper to list here all the considerations for practice the CTSG created and listed. Appendix E, however, provides a compilation of our strategies for the collaborative music classroom.

Suggestions for Future Research

I still have a number of related questions that will require further investigation and research. The first category of things I wonder about concerns the content of the CTSG. Could the CTSG have been more musical? I would like to reconvene a CTSG around the idea of chamber music collaboration, and have us perform as well as study together to see if the added layer of making music would add further dimension to our interactions. Also, I would like to incorporate some type of musicianship building exercises within a CTSG to see if the experience of being music learners together changes our dialogue. It could be as simple as learning a new song and bass line together each week, or composing music together, or even taking lessons together in advanced 
improvisation. I wonder if getting in touch with each other's music learner identities would give us additional things to talk about.

There are any number of possible subjects to study within elementary classroom music, many of which have been more clearly defined than collaboration. How would the CTSG change with the study of different topics, perhaps even a controversial one? More research should be done on how the topic or phenomenon under study intersects with the nature of the teacher community. Closely related to the issue of the CTSG topic is the idea of studying the implementation of specific pedagogical skills or methodologies. Further research should be done on using the teacher study group format to provide support for curricular or pedagogical implementation.

I wonder how the tone or tenor of our meetings might have changed had we stayed together longer. I would like to investigate a longer-term CTSG experience. It is possible that the good will and understanding we developed over seven meetings would sustain us in productive dialogue even on a challenging topic. It is possible that the protocol and video evidence would provide enough objective, contextual evidence on even controversial topics to help us build bridges between differing philosophies or methodologies. Explicitly probing these differences might result in a lot of learning for everyone, or possibly painful conflict. Further study of the CTSG format will help me know how much debate and disagreement is possible without losing its positive qualities of connection, support, and learning.

Different protocols and ways of structuring the meeting could be further investigated. There are other ways of organizing teacher study groups, such as lesson study (Stigler \& Hiebert, 1997) that might be effective. The experience would be 
different for the participants and comparisons between different organizing frameworks would be interesting. One of the most immediate applications I can see is to take video evidence a step further and have CTSG members first visit, then actually co-teach, one another's classes. This would be a fascinating way to explore contextual teacher learning.

The CTSG should also be studied with different configurations of participants: all teachers from the same level and the same district, teachers from the same district but different grade levels, teachers working in different types of music education, the inclusion of teachers outside music, and various combinations of preservice, beginning, and veteran teachers, just to name a few. I am especially curious about how a CTSG with student teachers would play out in terms of improving that last and very important preservice experience for undergraduates. What would change about the CTSG if all members were enrolled for credit, say, for a graduate course?

Also, would the CTSG be drastically different if I had not been a member/facilitator, rather, an ethnographer observing and documenting from the outsider perspective? The role of the university facilitator and organizer could be changed in a number of ways and studied. What if I had been more aggressive at the time about drawing connections between previous research and what we were learning in our meetings? Is the CTSG an appropriate place to disseminate research to practitioners? Or is the CTSG best left as an independent, knowledge-generating organization?

Our principles of collaboration could also be further studied, in terms of how they can be located or identified in the teaching and learning practice of others. Do these principles work for other students, in other areas of music (band, chorus, orchestra)? Can our principles be applied to other collaborative environments in music without a teacher 
(rock bands, chamber music)? Notwithstanding the principles, evidence of and for student musical collaboration is a rich area for future study.

\section{A Final Conversation}

Because this entire project has hinged on collaboration, conversation, and interaction, it seems strange to write my final words alone. Thus, I have written my concluding words in the form of a letter to the CTSG participants, to bring their presence back to these pages.

Dear Marlene, Andrea, and Karly,

As I reflect today on the story of the CTSG, I have been wondering if the experience will continue to have meaning for you, and how it will possibly matter to other music teachers. Will a study of the experiences of three upstate New York elementary music teachers and one university professor in a collaborative teacher study group have validity for anyone who was not there, who did not watch the videos, laugh, learn, and eat pizza with us?

Our journey was so entwined with our own unique circumstances, but surely anyone can relate to at least a part of it. Karly, in a recent email, told me:

It was very interesting to think about the journey of each CTSG member throughout the experience. We all started with very different mindsets, and these mindsets were definitely based on our prior experiences and immediate needs. What was even more interesting was to think about how we influenced each other, which led to us refining our views and even "converging on a shared conceptual understanding." Since the three stories are so different, it would be hard for any educator not to identify with at least one of the three! (Karly, personal email, November 3, 2008)

On the one hand, I don't really care if the results from this study are "valid" in the positivist sense, meaning they can be generalized to other populations. I learned about 
collaboration by collaborating with you. I had an important experience in the CTSG, and I know you did too. I fully believe others can learn from our lessons.

On the other hand, Dr. Patti Lather, a professor and researcher at Ohio State, has several post-positivist definitions of validity in research. For Lather, one way to determine if a study has validity is to assess if the study was a catalyst for change, for instigating action and taking further steps.

And the story of the CTSG is definitely a catalyst for change.

This study is a catalyst for change in the way music teacher professional development might be considered. Professional development should not be about sitting in a room, listening to an expert, and falling asleep because the topic is irrelevant. You three teachers, and your colleagues working hard in classrooms around the world, have collective wisdom greater than any one expert can deliver in a workshop. If teachers are given the opportunity to watch one another and have real, meaningful conversations about music teaching and learning, it will be like tapping into the mother lode of golden information. Not one of us has all the answers but there's very little we can't figure out together, if we have the places and ways in which to talk productively.

Professional development should be changed to allow teachers to understand more about how students learn music, and build the professional knowledge base for our profession. The workshops on rhythm games are still fun, and I know we'll enjoy playing the games together when we're at our next conference. But I want professional development to be far deeper and longer lasting. As professionals, we deserve more.

This study is also a catalyst for change in how music teachers view student musical collaboration. I'm sure music teachers everywhere have their own ideas about 
collaboration, just as each of us started with our own definition of collaboration. In the beginning, we said we wanted our classrooms to be filled with interactive music-making, creativity, and caring. As we met, we talked about how we want our students to make music, and learn musical skills so they can be independent musicians who take ownership of their creations. We kept trying to figure out how to help our students work together, and how to help our students work with us, even though our students and teaching situations are so different.

It soon became clear, though, that we could all get behind the definitions we came up with as a group. Collaboration is big enough, and important enough, for all of us to use as an overarching concept. Our principles of collaboration give us, and other music teachers, a catalyst for change. We can use collaboration as one more approach or means to the end, with the end being all those worthy things we want for our students.

Finally I want to thank you for allowing me into your lives. You trusted me to put this group together and tell your individual stories. You read and re-read everything even when it was raw and untested, because you are interested, intellectual people who care about our profession and making it better. I admire you for being teachers with the courage to join with me on this project. I admire you for having the drive to get up and teach Monday through Friday, and to think about teaching in the middle of the night. And I really miss our meetings. Especially the food.

Collaboratively yours,

Ann Marie 
Appendix A

Collaborative Consultancy Protocol

\begin{tabular}{|c|c|c|c|}
\hline Purpose & $\begin{array}{l}\text { Kinds of Work } \\
\text { Presented }\end{array}$ & $\begin{array}{l}\text { Brief Summary of } \\
\text { Steps }\end{array}$ & Facilitator Role \\
\hline $\begin{array}{l}\text { 1. To give the } \\
\text { presenter a fresh } \\
\text { perspective on } \\
\text { student work, } \\
\text { particularly when } \\
\text { the presenter is } \\
\text { genuinely } \\
\text { wondering about, or } \\
\text { baffled by, the work } \\
\text { or student(s). } \\
2 \text {. To promote } \\
\text { understanding about } \\
\text { particular students } \\
\text { as music learners, to } \\
\text { examine aspects of } \\
\text { collaboration in the } \\
\text { classroom, and to } \\
\text { spark thinking about } \\
\text { teaching and } \\
\text { learning issues } \\
\text { related to } \\
\text { collaboration that } \\
\text { may apply to other } \\
\text { students and } \\
\text { classrooms. }\end{array}$ & $\begin{array}{l}\text { Video of students in } \\
\text { the music } \\
\text { classroom, selected } \\
\text { by the presenting } \\
\text { teacher. }\end{array}$ & $\begin{array}{l}\text { 1. Presenter } \\
\text { provides no context } \\
\text { initially. } \\
\text { 2. CTSG members } \\
\text { describe what they } \\
\text { see on the video. } \\
\text { 3. CTSG members } \\
\text { raise a variety of } \\
\text { questions about the } \\
\text { work. } \\
\text { 4. CTSG members } \\
\text { speculate about and } \\
\text { interpret the } \\
\text { students' musical } \\
\text { behavior and look } \\
\text { for evidence to } \\
\text { support these } \\
\text { assertions. } \\
\text { 5. Presenter shares } \\
\text { context and reflects } \\
\text { on the conversation } \\
\text { she has heard. } \\
\text { 6. CTSG members } \\
\text { discuss implications } \\
\text { for the presenter's } \\
\text { practice, and for } \\
\text { their own practice. } \\
7 . \text { Everyone debriefs } \\
\text { the process, through } \\
\text { written reflection, or } \\
\text { group discussion. }\end{array}$ & $\begin{array}{l}\text { 1. Before the } \\
\text { protocol: help the } \\
\text { presenter understand } \\
\text { she will have no } \\
\text { input into the } \\
\text { protocol discussion } \\
\text { until late in the } \\
\text { process; no } \\
\text { contextual } \\
\text { information is given } \\
\text { to the group before } \\
\text { they begin their } \\
\text { discussion. } \\
\text { 2. During the } \\
\text { protocol: Guide } \\
\text { CTSG members in } \\
\text { the difference } \\
\text { between description, } \\
\text { interpretation, and } \\
\text { evaluation, to enable } \\
\text { them to describe the } \\
\text { work without } \\
\text { judging it. Help } \\
\text { CTSG members } \\
\text { avoid advice giving; } \\
\text { enable reflection on } \\
\text { teaching and } \\
\text { learning, with a } \\
\text { focus on } \\
\text { collaboration } \\
\text { between students. }\end{array}$ \\
\hline
\end{tabular}


Appendix B

Dilemma Consultancy Protocol

\begin{tabular}{|c|c|c|c|}
\hline Purpose & $\begin{array}{l}\text { Kinds of Work } \\
\text { Presented }\end{array}$ & $\begin{array}{l}\text { Brief Summary of } \\
\text { Steps }\end{array}$ & Facilitator Role \\
\hline $\begin{array}{l}\text { 1.To help the } \\
\text { presenter think } \\
\text { more expansively } \\
\text { about a } \\
\text { professional } \\
\text { dilemma she is } \\
\text { facing through } \\
\text { offering other } \\
\text { points of views and } \\
\text { other possibilities. }\end{array}$ & $\begin{array}{l}\text { Video of students } \\
\text { in the music } \\
\text { classroom, selected } \\
\text { by the presenting } \\
\text { teacher. }\end{array}$ & $\begin{array}{l}\text { 1. Presenter gives } \\
\text { overview of the } \\
\text { dilemma. } \\
\text { 2. Presenter provides a } \\
\text { focusing question (i.e., } \\
\text { "What aspects of this } \\
\text { behavior/activity/event } \\
\text { could I change to help } \\
\text { the students learn } \\
\text { more together?") } \\
\text { 3. CTSG members ask } \\
\text { clarifying and probing } \\
\text { questions. } \\
\text { 4. CTSG members } \\
\text { provide feedback } \\
\text { designed to give the } \\
\text { presenter new } \\
\text { perspectives/ideas. } \\
\text { 5. Presenter responds. } \\
\text { 6. CTSG members } \\
\text { discuss implications } \\
\text { for the presenter's } \\
\text { practice, and for their } \\
\text { own practice. } \\
\text { 7. Everyone debriefs } \\
\text { the process, through } \\
\text { written reflection, or } \\
\text { group discussion. }\end{array}$ & $\begin{array}{l}\text { 1. Before the } \\
\text { protocol: works } \\
\text { with the presenter to } \\
\text { shape the dilemma } \\
\text { and discuss how it } \\
\text { will be shared with } \\
\text { the group. It is } \\
\text { critical that the } \\
\text { presenter thinks a } \\
\text { solution to dilemma } \\
\text { is possible but } \\
\text { doesn't know what } \\
\text { it is, and that the } \\
\text { presenter is willing } \\
\text { to try changes in } \\
\text { practice to resolve } \\
\text { the dilemma. } \\
\text { 2. During the } \\
\text { protocol: Helps } \\
\text { CTSG members } \\
\text { appreciate } \\
\text { difference between } \\
\text { probing questions } \\
\text { and those that } \\
\text { merely disguise } \\
\text { recommendations } \\
\text { imply solutions. }\end{array}$ \\
\hline
\end{tabular}




\section{Appendix C}

Representative Questions for Semi-structured Interviews with CTSG Participants First Interview:

1. How did you experience professional development in the past?

2. How have you incorporated things you have learned in professional development into your teaching practice?

3. How do you view collaboration with other teachers?

4. How do you define collaboration in the elementary music classroom?

5. How do you perceive collaboration as occurring in your particular elementary music classroom?

6. How have you experienced student musical collaboration? Second Interview:

1. How do you perceive the experience of working in the CTSG?

2. How have you thought about collaboration in the weeks between the meetings?

3. How are your students currently experiencing collaboration in the elementary school music classroom? How do you perceive any changes in your teaching or in your students' learning? 


\section{Appendix D}

\section{Coding Categories}

RQ1. How do the participants describe their experiences in the CTSG?

* look for broad, general, open descriptors*

RQ1a. (For the profession at large): CTSG as professional development that supports teacher learning/change in practice

RQ1aKarly

RQ1aAndrea

RQ1aMarlene (these subheadings go to the findings for each individual teacher's story)

RQ1b. (For the profession) CTSG as professional development that combats isolation/increases satisfaction

RQ1bKarly

RQ1bAndrea

RQ1bMarlene

RQ1c. CTSG as professional development that improves music education of students
RQ1cKarly
RQ1cAndrea
RQ1cMarlene

RQ1d. CTSG as professional development that is otherwise effective

RQ1dKarly

RQ1dAndrea

RQ1dMarlene

RQ2. How has the focus on collaboration in the CTSG changed their teaching practice?

RQ2a. how has the focus on collaboration AS A TOPIC OF STUDY in the CTSG changed their teaching practice?

RQ2b. how has the focus on collaboration AS AN EVENT OR PHENOMENON unique to the CTSG changed their teaching practice? 
RQ3. What can these music teachers tell us about collaboration?

RQ3a. about their own collaboration? (generally, for the profession)

RQ3aKarly

RQ3aAndrea

RQ3aMarlene (specifically, for their individual stories)

RQ3b. about that of their students? (generally, for the profession)

RQ3c. collaboration enhancing student learning

RQ3d. difficulties in student collaboration

RQ3e. changes in defining, viewing or perceiving student collaboration

RQ3f. markers of collaboration in the classroom

RQ3g. facilitating collaboration in the classroom

RQ3h. collaboration as starting a generative process

RQ3i. collaboration related to student creativity, independence, self-expression

RQ3bKarly

RQ3bAndrea

RQ3bMarlene (specifically, for their individual stories)

*keep these broad, general, so as not to narrow findings to applicability in only these 3 teachers' classrooms

\section{Related to Definitions of Collaboration-}

Use these for coding Teacher Collaboration AND Student Collaboration (as specified)

D1. "people negotiate, construct and share relevant meanings, participate in coordinated, synchronous activity and pursue a continued attempt to maintain new concepts" (Roschelle \&Teasley 's 1995 definition)

D1a. negotiation and construction of shared meanings

D1b. continued attempts to maintain new concepts

D1c. evidence that CTSG interactions influence teacher learning

D2. "a class of 25 can collaborate as long as interactivity with peers influences the students' cognitive processes, and as long as there is a degree of negotiation and synchronous action." (Dillenbourg 1999)

D2a. evidence of interactivity with PEERS influencing processes of musicmaking D2b. collaboration between students and teacher

D3. "Symmetry of action, status and division of labor" (Dillenbourg 1999)

D3a. Symmetry in CTSG 
D3b. Symmetry in classroom 


\section{Appendix E}

Strategies For the Collaborative Music Classroom

\begin{tabular}{|c|c|}
\hline $\begin{array}{l}\text { A Sampling of Types of } \\
\text { Collaboration in the } \\
\text { Elementary Music Classroom }\end{array}$ & 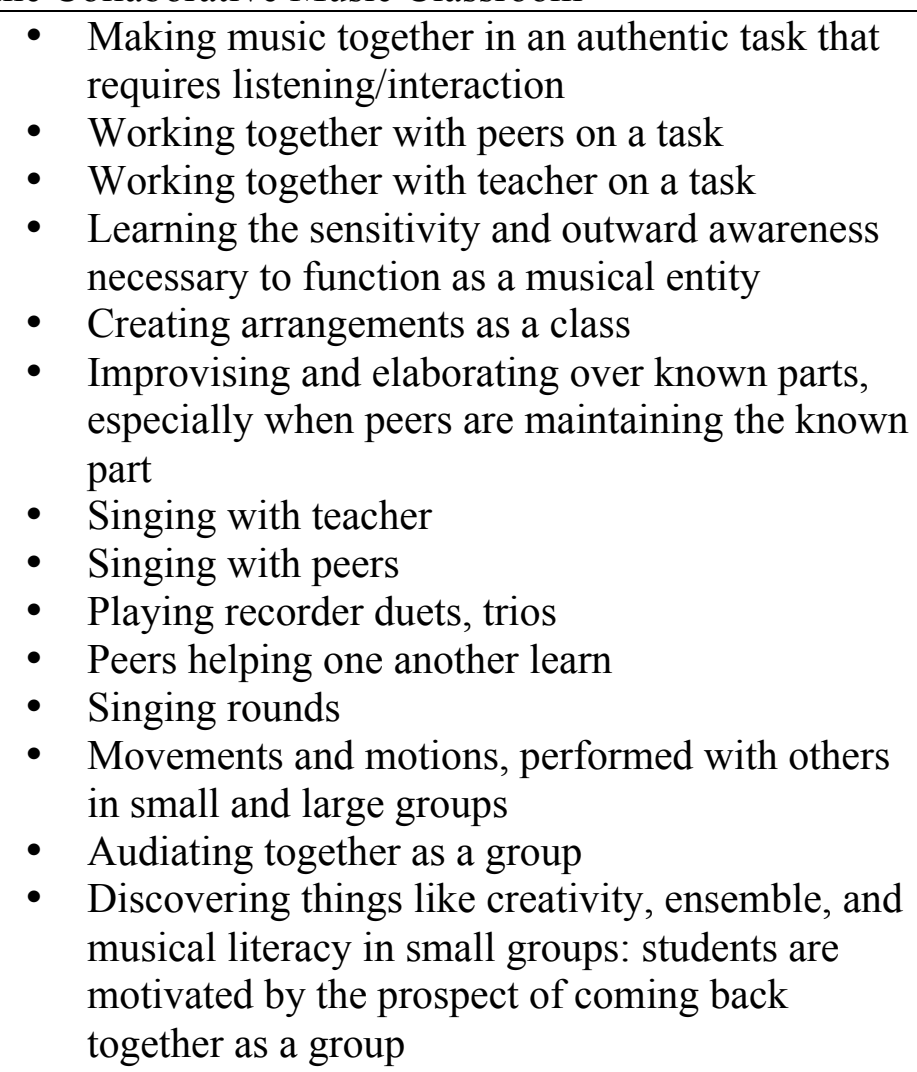 \\
\hline $\begin{array}{l}\text { Strategies for Creating a } \\
\text { Musically Collaborative } \\
\text { Classroom }\end{array}$ & $\begin{array}{l}\text { - Create a lunchtime or recess practice period for a } \\
\text { few students to come in and try out musical } \\
\text { ideas. These students become "ringers" or } \\
\text { "leaders" in the full group and able to assist in } \\
\text { larger-group collaborations such as creating } \\
\text { arrangements, etc. } \\
\text { - Teach a small group a background/bass } \\
\text { line/rhythmic part and make them the "rock" that } \\
\text { others will experiment over. Let them teach } \\
\text { others their part so students move in and out of } \\
\text { the "rock" part } \\
\text { - Once students are solid as a class on a chant or } \\
\text { tune (think of "2-4-6-8" chant, for example) try } \\
\text { the following to enhance collaboration: } \\
\text { O Insert improvised phrases (rhythmic, } \\
\text { melodic, instrumental) within the piece } \\
\text { for creative buy-in, personalization } \\
\text { - Ask students to create an arrangement in } \\
\text { a large or small group }\end{array}$ \\
\hline
\end{tabular}




\begin{tabular}{|c|c|}
\hline & $\begin{array}{l}\circ \text { Add an ostinato or counterpart. Ask } \\
\text { students to help create this } \\
\circ \text { Ask students to create an accompanying } \\
\text { movement } \\
\circ \quad \text { Pull "leaders" to help reinforce/reteach } \\
\text { others } \\
\circ \text { Constantly ask for ideas and feedback } \\
\circ \quad \text { Students in small groups can create their } \\
\text { own final line or coda } \\
\text { - "Group-think" activities like audiation help } \\
\text { students to internalize music but at the same time } \\
\text { function as a group, in sync. } \\
\text { Have students sing to each other, face-to-face, } \\
\text { knee-to-knee (knee-to-knee helps maintain a } \\
\text { close yet comfortable, reassuring distance.) } \\
\text { Observe students' eye contact. }\end{array}$ \\
\hline $\begin{array}{l}\text { Progressions To Help } \\
\text { Students Collaborate }\end{array}$ & 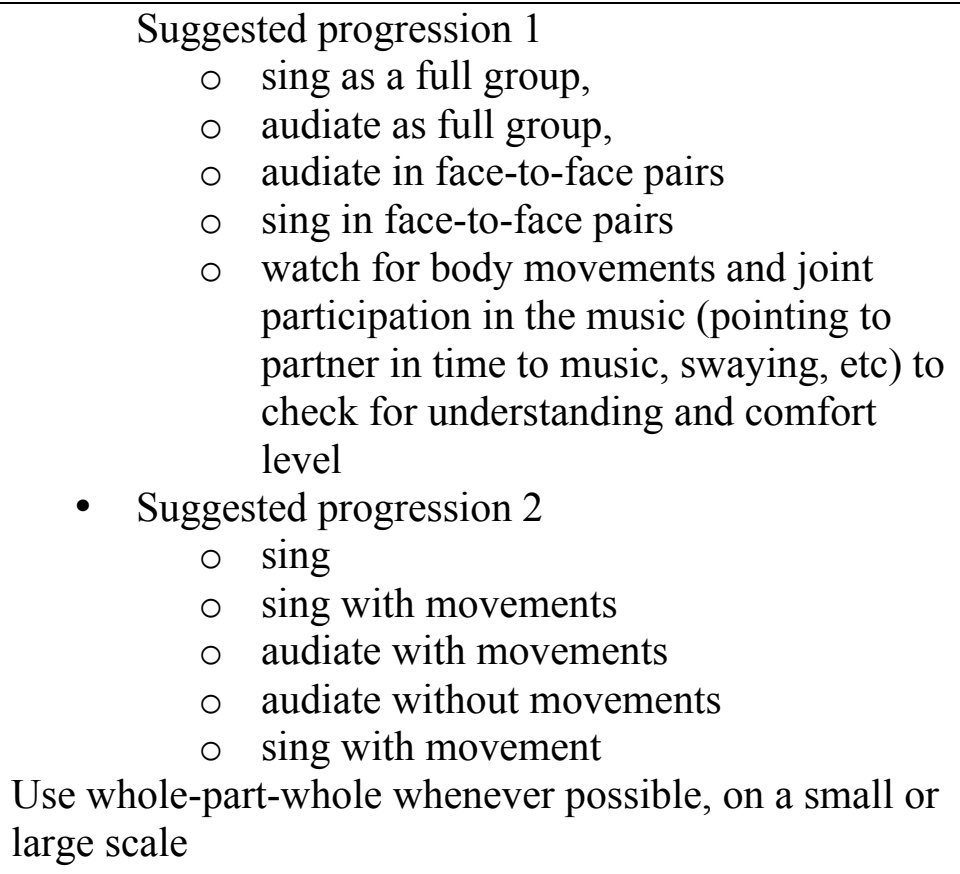 \\
\hline $\begin{array}{l}\text { Explicitly Teaching or } \\
\text { Modeling Collaborative } \\
\text { Behaviors }\end{array}$ & $\begin{array}{l}\text { - "Can we mouth the words?" } \\
\text { - "Can we watch each other?" } \\
\text { - "Try [playing, singing] and watch someone else } \\
\text { in the class, or across the room" } \\
\text { "Watch your classmates and see how they're } \\
\text { doing it", "Watch your classmates to stay with } \\
\text { them" } \\
\text { - "Play to someone near you" (or across the room) } \\
\text { - "Check your beat with everyone else in the }\end{array}$ \\
\hline
\end{tabular}




\begin{tabular}{|c|c|}
\hline & $\begin{array}{l}\text { class" } \\
\text { - Model collaboration using think-alouds, role } \\
\text { playing, fish bowl } \\
\text { - Use puzzle pieces as a metaphor- "we need every } \\
\text { piece"- individuals and groups are responsible } \\
\text { for something that when combined, will create } \\
\text { something bigger than the sum of its parts }\end{array}$ \\
\hline $\begin{array}{l}\text { Strategies for Creating a More } \\
\text { Emotionally/Socially } \\
\text { Collaborative Classroom }\end{array}$ & $\begin{array}{l}\text { - Group goals can be fostered by teacher } \\
\text { comments: (use "we" language) } \\
\text { o "We're working for that perfect } \\
\text { ending!" } \\
\text { "We want to do this really well so } \\
\text { we can take it to the next level!" } \\
\text { "What should we do/add/try next?" } \\
\text { "We'll all have success if we all sing } \\
\text { it in our heads" } \\
\text { "We can put this together in a big } \\
\text { group after you work in your small } \\
\text { groups" } \\
\text { "We're going to be part of something } \\
\text { big!" } \\
\text { - Students respond to teacher praise and pride in } \\
\text { their peer role models with an attitude of : "it's } \\
\text { okay to try, it's okay to fail" } \\
\text { Compliment each student on their collaboration } \\
\text { as they leave } \\
\text { - Watch for collaboration and pointing it out } \\
\text { Use (and inspire) their natural desire for } \\
\text { partnership: music buddies, working with } \\
\text { friends, working in groups }\end{array}$ \\
\hline $\begin{array}{l}\text { Strategies for Creating } \\
\text { Student "Buy-in" in the } \\
\text { Collaborative Classroom }\end{array}$ & 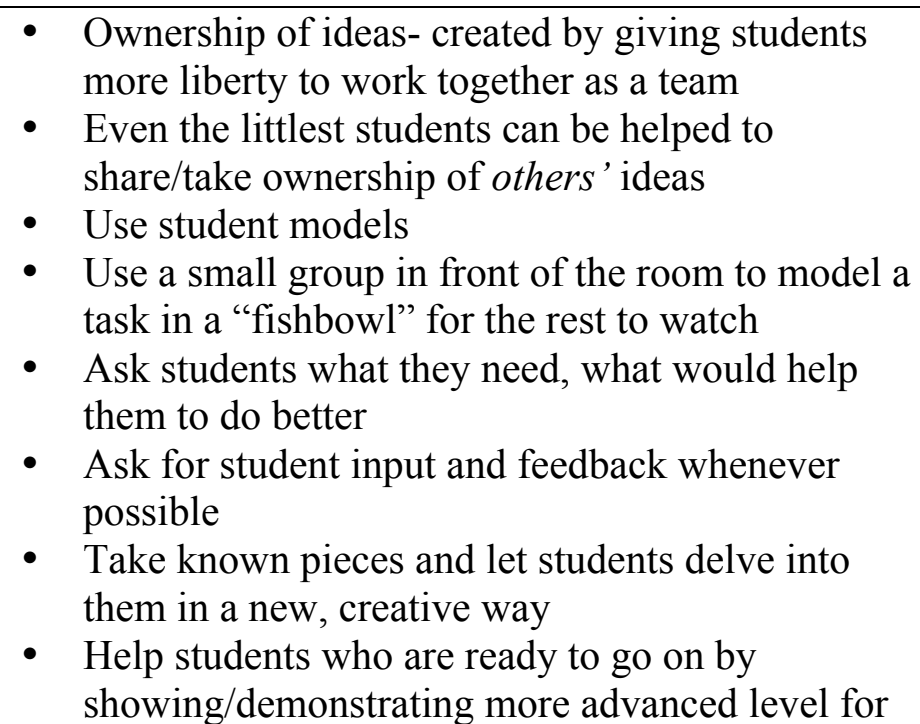 \\
\hline
\end{tabular}




\begin{tabular}{|c|c|}
\hline & $\begin{array}{l}\text { others; this also seems to help students who are } \\
\text { not ready yet to know what they're working for }\end{array}$ \\
\hline $\begin{array}{l}\text { Challenges teachers find } \\
\text { when facilitating } \\
\text { collaboration in the } \\
\text { elementary music classroom }\end{array}$ & $\begin{array}{l}\text { - Students who vocally disagree and try to get } \\
\text { allies } \\
\text { - Students who have checked out: the silent non- } \\
\text { thinker, "invisibles" } \\
\text { - Students who have given up trying to find their } \\
\text { voice, and seemingly don't care } \\
\text { - Students lacking problem-solving and } \\
\text { compromise strategies } \\
\text { - Possible negative impression given when } \\
\text { classroom seems chaotic } \\
\text { - Finding a balance between joy, freedom, and } \\
\text { control } \\
\text { - Difficult to find opportunities for students to } \\
\text { offer feedback, ideas, suggestions } \\
\text { Little time to ask broad, guiding questions; } \\
\text { difficulties in eliciting enough varied responses } \\
\text { as well (i.e. hearing from many voices) } \\
\text { Identifying varying needs of students and } \\
\text { differentiating the classroom activities enough to } \\
\text { meet these needs } \\
\text { Finding efficient ways to create groups or choose } \\
\text { partners }\end{array}$ \\
\hline Things to Watch For & $\begin{array}{l}\text { - } \quad \text { Students turning to look at each other } \\
\text { - Students' eye contact, direction of face, body } \\
\text { - } \quad \text { Students turning ears to music } \\
\text { - } \quad \text { Kids "making impressions" on each other- and } \\
\text { - } \text { how to use that for learning } \\
\text { - } \quad \text { Mudents identifying strengths of self and others } \\
\text { Off -topic chit-chat (may be helpful in } \\
\text { - } \quad \text { Undablishing relationships) } \\
\text { need of teacher, not their own }\end{array}$ \\
\hline
\end{tabular}




\author{
Appendix F \\ Institutional Review Board (IRB) Exemption Letter
}

Behavioral Sciences Institutional Review Board (IRB)

540 East Liberty Street, Suite 202, Ann Arbor, MI 48104-2210

phone (734) 936-0933

fax (734) 998-9171

irbhsbs@umich.edu

To: Ann Marie Stanley

From:

James Sayer

Colleen Seifert

Ce:

Colleen Conway

Ann Marie Stanley

Subject: Notice of Exemption for [HUM00019749]

\title{
SUBMISSION INFORMATION:
}

Title: The Experiences of Elementary Music Teachers Participating in a Collaborative Teacher Study Group

Full Study Title (if applicable): The Experiences of Elementary Music Teachers

Participating in a Collaborative Teacher Study Group

Study eResearch ID: HUM00019749

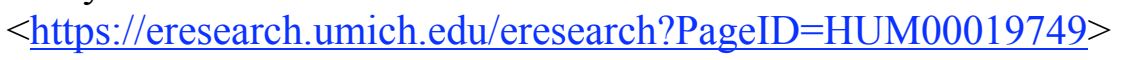

Date of this Notification from IRB: $3 / 21 / 2008$

Date of IRB Exempt Determination: 3/21/2008

UM Federalwide Assurance: FWA00004969 expiring on 5/10/2009

OHRP IRB Registration Number(s): IRB00000246

\section{IRB EXEMPTION STATUS:}

The IRB Behavioral Sciences has reviewed the study referenced above and determined that, as currently described, it is exempt from ongoing IRB review, per the following federal exemption category:

EXEMPTION \#2 of the 45 CFR 46.101.(b): 
Research involving the use of educational tests (cognitive, diagnostic, aptitude, achievement), survey procedures, interview procedures or observation of public behavior, unless: (i) information obtained is recorded in such a manner that human subjects can be identified, directly or through identifiers linked to the subjects; and (ii) any disclosure of the human subjects' responses outside the research could reasonably place the subjects at risk of criminal or civil liability or be damaging to the subjects' financial standing, employability, or reputation.

Note that the study is considered exempt as long as any changes to the use of human subjects (including their data) remain within the scope of the exemption category above. Any proposed changes that may exceed the scope of this category, or the approval conditions of any other non-IRB reviewing committees, must be submitted as an amendment through eResearch.

Although an exemption determination eliminates the need for ongoing IRB review and approval, you still have an obligation to understand and abide by generally accepted principles of responsible and ethical conduct of research. Examples of these principles can be found in the Belmont Report as well as in guidance from professional societies and scientific organizations.

\section{SUBMITTING AMENDMENTS VIA ERESEARCH:}

You can access the online forms for amendments in the eResearch workspace for this exempt study, referenced above.

\section{ACCESSING EXEMPT STUDIES IN eRESEARCH:}

Click the "Exempt and Not Regulated" tab in your eResearch home workspace to access this exempt study.

\section{James Sayer}

Co-chair, IRB Behavioral Sciences Colleen Seifert

Co-chair, IRB Behavioral Sciences 


\section{References}

Achinstein, B. (2002). Conflict among community: The micropolitics of teacher collaboration. Teachers College Record, 104(3), 421-455.

Allen, D., \& Blythe, T. (2004). The facilitator's book of questions: Tools for looking together at student and teacher work. New York: Teachers College Press.

Azmitia, M., \& Perlmutter, M. (1989). Social influences on children's cognition: State of the art and future directions. In H. W. Reese (Ed.), Advances in child development and behavior (Vol. 22, pp. 89-144). New York: Academic Press.

Barnes, N. (2000). Teachers teaching teachers. Education Week, 19(19), 38-42.

Barrett, J. (2006). Recasting professional development for music teachers in an era of reform. Arts Education Policy Review, 107(6), 19-28.

Barron, B. (2000). Achieving coordination in collaborative problem-solving groups. The Journal of the Learning Sciences, 9(4), 403-436.

Bauer, W. I. (2007). Research on professional development for experienced music teachers. Journal of Music Teacher Education, 17(1), 12-21.

Berg, M. H. (1997). Social construction of musical experience in two high school chamber music ensembles. (Doctoral Dissertation, Northwestern University, 1997). Dissertation Abstracts International, 58, 04A.

Blum, D. (1986). The art of quartet playing: The Guarneri quartet in conversation with David Blum. Ithaca, NY: Cornell University Press.

Boghossian, P. (2006). Fear of knowledge: Against relativism and constructivism. Oxford,UK: Clarendon.

Borko, H. (2004). Professional development and teacher learning: Mapping the terrain. Educational Researcher, 33, 3-15.

Bransford, J.D., Brown, A.L., \& Cocking, R.R. (Eds.). (2000). How people learn: Brain mind, experience, and school. Washington, DC: National Academy Press.

Brown, J.S., Collins, A., \& Duguid, P. (1989). Situated cognition and the culture of learning. Educational Researcher, 18, 32-42. 
Brown, J.S., \& Duguid, P. (1996). Stolen knowledge. In H. McLellen (Ed.), Situated learning perspectives (pp. 47-56). Englewood Cliffs, NJ: Educational Technology Publications.

Bruffee, K. A. (1999). Collaborative learning: Higher education, interdependence, and the authority of knowledge (2nd ed.). Baltimore: Johns Hopkins University Press.

Bruner, J. (1960). The process of education. Cambridge, MA: Harvard University Press.

Burnard, P. (2002). Investigating children's meaning-making and the emergence of musical interaction in group improvisation. British Journal of Music Education (19)2, 157-172.

Chinn, C.A., O’Donnell, A.M., \& Jinks, T.S. (2000). The structure of discourse in collaborative learning. The Journal of Experimental Education, 69(1), 77-97.

Cochran-Smith, M., \& Donnell, K. (2006). Practitioner inquiry: Blurring the boundaries of research and practice. In J.L. Green, G. Camilli, \& P.B. Elmore (Eds.), Handbook of complementary methods in education research (pp. 503-518). Washington, D.C.: AERA.

Cognition and Technology Group at Vanderbilt. (1997). The Jasper Project: Lessons in curriculum, instruction, assessment, and professional development. Mahwah, NJ: Lawrence Erlbaum Associates, Inc.

Colwell, R., \& Richardson, C. (Eds.) (2002). The new handbook of research on music teaching and learning. Oxford: Oxford University Press.

Conway, C.M. (2003). Ongoing professional development. In C.M. Conway, Ed., Great beginnings for music teachers: Mentoring and supporting new teachers (pp. 151166). Reston, VA: MENC: The National Association for Music Education.

Conway, C. M., Albert, D., Hibbard, S., \& Hourigan, R. (2005a). Arts education and professional development. Arts Education Policy Review, 107(1), 3-9.

Conway, C. M., Albert, D., Hibbard, S., \& Hourigan, R. (2005b). Voices of music teachers regarding professional development. Arts Education Policy Review, 107(1), 11-14.

Cook, D.N., \& Brown, J.S. (1999). Bridging epistemologies: The generative dance between organizational knowledge and organizational knowing. Organization Science (10)4, 381-400.

Cousin, G., \& Deepwell, F. (2005). Designs for network learning: A communities of practice perspective. Studies in higher education, 30(1), 57-66. 
Curry, M. (2008). Critical friends groups: The possibilities and limitations embedded in teacher professional communities aimed at instructional improvement and school reform. Teachers College Record, 110(4), 733-774.

Custadero, L.A. (2002). Seeking challenge, finding skill: Flow experience and music education. Arts Education Policy Review, 103(3), 3-9.

Davidson, J.W., \& Good, J.M.M. (2002) Social and musical coordination between members of a string quartet: An exploratory study. Psychology of Music, 30: 186201.

DeLorenzo, L.C. (1989). A field study of sixth-grade students' creative music problemsolving processes. Journal of Research in Music Education, 37(3), 188-200.

Dewey, J. (1938). Experience and education. New York: Collier Books, Macmillan Publishing Company.

Dillon, T. (2003). Collaborating and creating on music technologies. International Journal of Educational Research 39 (8), 893-897.

Dillenbourg, P. (1999). What do you mean by collaborative learning?. In P. Dillenbourg (Ed.), Collaborative-learning: Cognitive and computational approaches (pp. 119). Oxford: Elsevier.

Dolloff, L. A. (1996). Expertise in choral music education: Implications for teacher education (Doctoral dissertation, University of Toronto, 1994). Dissertation Abstracts International, 56(07), 2600.

Duke, R.A. (1999). Measures of instructional effectiveness in music research. Bulletin of the Council for Research in Music Education, 143, 1-48.

Engestrom, Y. (1996). Perspectives on activity theory. Cambridge: Cambridge University Press.

Feiman-Nemser, S. (2001). From preparation to practice: Designing a continuum to strengthen and sustain teaching. Teachers College Record, 103(6), 1013-1055.

Fischer, F., \& Mandl, H. (2005). Knowledge convergence in computer-supported collaborative learning: The role of external representation tools. The Journal of the Learning Sciences, 14(3), 405-441.

Forman, E.A., Stein, M.K., Brown, C., Larreamendy-Joerns, J. (1995). The socialization of mathematical thinking: The role of institutional, interpersonal, and discursive contexts. Paper presented at the $77^{\text {th }}$ Annual Meeting of the American Educational Research Association, San Francisco.

Friedrichs, C. (2001). The effect of professional growth opportunities as determined by 
California public high school instrumental music teachers (Doctoral dissertation, University of San Diego, 2001). Dissertation Abstracts International, 62(03), 955.

Garet, M. S., Porter, A. C., Desimone, L., Birman, B. F., \& Yoon, K. S. (2001). What makes professional development effective? Results from a national sample of teachers. American Educational Research Journal, 38(4), 915-945.

Gokhale, A.A. (1995). Collaborative learning enhances critical thinking. Journal of Technology Education, 7(1), 22-30.

Gordon. E. (1997). Learning sequences in music, skill, content and patterns: A music learning theory. Chicago: GIA Publications.

Grossman, P., Wineburg, S., \& Woolworth, S. (2000). What makes teacher community different from a gathering of teachers?. Seattle: University of Washington Center for the Study of Teaching and Policy.

Guba, E.G., \& Lincoln, Y. (2005). Paradigmatic controversies, contradictions, and emerging confluences. In. N.K. Denzin \& Y.S. Lincoln (Eds.), The Sage handbook of qualitative research $\left(3^{\text {rd }}\right.$ ed., pp. 183-215). Thousand Oaks, CA: Sage.

Haack, P. (2003). Challenges faced by beginning music teachers. In C.M. Conway, Ed., Great beginnings for music teachers: Mentoring and supporting new teachers (pp. 9-26). Reston, VA: MENC: The National Association for Music Education.

Hammel, A.M. (2007). Professional development research in general education. Journal of Music Teacher Education, 17(1), 22-32.

Hanley, B., \& Montgomery, J. (2002). Contemporary curriculum practices and their theoretical bases. In R. Colwell \& C. Richardson (Eds.), The new handbook of research on music teaching and learning (pp. 759-785). Oxford: Oxford University Press.

Hanley, B., \& Montgomery, J. (2005). Challenges to music education: Curriculum reconceptualized. Music Educators Journal, 91(4), 17-20.

Hargreaves, A. (1995). Development and desire: A postmodern view. In T.R. Guskey, \& M. Huberman (Eds.), Professional development in education: New paradigms and practices (pp. 9-34). New York: Teachers College Press.

Hoffman, J. A. (1991). Computer-aided collaborative music instruction. Harvard Educational Review, 61(3), 270-78.

Horn, I.S. (2005). Learning on the job: A situated account of teacher learning in high school mathematics departments. Cognition and Instruction, 23(2), 207-236. 
Junda, M. E. (1994). A model in-service music teacher education program. Journal of Music Teacher Education, 3(2), 6-20.

King, E.C. (2004). Collaboration and the study of ensemble rehearsal. Paper presented at The Eighth International Conference on Music Perception and Cognition, Evanston, IL, August.

King, E.C. (2006). The roles of student musicians in quartet rehearsals. Psychology of Music, 34(2), 262-282.

Kovalainen, M., \& Kumpalainen, K. (2007). The social construction of participation in an elementary classroom community. International Journal of Educational Research, 46, 141-158.

Kynigos, C., \& Theodossopoulou, V. (2001). Synthesizing personal, interactionist and social norms perspectives to analyze collaborative learning in the classroom in the context of a computer-based innovation program. Journal of Classroom Interaction, 36(2), 63-73.

Lather, P. (2003). Issues of validity in openly ideological research. In Y.S. Lincoln \& N.K. Denzin (Eds.), Turning points in qualitative research: Tying knots in a handkerchief (pp. 185-215).

Lave, J., \& Wenger, E. (1991). Situated learning: Legitimate peripheral participation. New York: Cambridge University Press.

Little, J.W. (1993). Teachers' professional development in a climate of educational reform. Educational Evaluation and Policy Analysis, 15(2), 129-151.

Little, J.W. (2003). Inside teacher community: Representations of classroom practice. Teachers College Record, 105(6), 913-945.

Little, J.W., Gearhart, M., Currie, M., Kafka, J. (2003). Looking at student work for teacher learning, teacher community, and school reform. Phi Delta Kappan, 85(3), 184-192.

Luce, D. (2001). Collaborative learning in music education: A review of the literature. Update: Applications of Research in Music Education, 19(2), 20-25.

MacDonald, R., \& Miell, D. (2004). Creative collaborations: Young people's musical interactions. Paper presented at The Eighth International Conference on Music Perception and Cognition, Evanston, IL, August.

McLaughlin, M.W., \& Talbert, J.E. (2001). Professional communities and the work of high school teaching. Chicago: University of Chicago Press. 
McLaughlin, M.W., \& Talbert, J.E. (2006). Building school-based teacher learning communities: Professional strategies to improve student achievement. New York: Teachers College Press.

Maykut, P., \& Morehouse, R. (1994). Beginning qualitative research: A philosophic and practical guide. London: Routledge Falmer.

Mead, G.H. (1934). Mind, self, and society. Chicago: University of Chicago Press.

Mezirow, J. (2000). Learning to think like an adult. In J. Mezirow and Associates, Learning as transformation: Critical perspectives on a theory in progress (pp. 333). San Francisco: Jossey-Bass.

Music Educators National Conference (1994). Perspectives on implementation: Arts education standards for America's students. Reston, VA: MENC: The National Association for Music Education.

Neimeyer, G.J. (1993). Constructivist assessment: A casebook. Thousand Oaks, CA: Sage.

Noddings, N. (1996). On community. Educational Theory, 46(3). 245-266.

Palincsar, A.S. (1998). Social constructivist perspectives on teaching and learning. Annual Review of Psychology, 49, 345-375.

Palincsar, A.S., \& Herrenkohl, L.R. (2002). Designing collaborative learning contexts. Theory Into Practice, 41(1), 26-32.

Palincsar, A.S., Magnusson, S.J., Marano, N., Ford, D., \& Brown, N. (1998). Designing a community of practice: Principles and practices of the GiSML community. Teaching and Teacher Education, 14(1), 5-19.

Papert, S. (1993). The children's machine: rethinking school in the age of the computer. New York: Basic Books.

Patton, M. Q. (2002). Qualitative research and evaluation methods (3rd ed.). Thousand Oaks, Ca: Sage.

Penuel, W.R., Fishman, B.J.,Yamaguchi, R., \& Gallagher, L.P. (2007). What makes professional development effective? Strategies that foster curriculum implementation. American Educational Research Journal, 44(4), 921-958.

Penuel, W. R., Riel, M., Frank, K. A., \& Krause, A. (2009, in press). Analyzing teachers' professional interactions in a school as social capital: A social network approach. Teachers College Record, 111(1). 
Perret-Clermont, A.N., Perret, J.F., \& Bell, N. (1991). The social construction of meaning and cognitive activity in elementary school children. In Resnick, L.B., Levine, J.M., \& Teasley, S.D. (Eds.), Perspectives on socially shared cognition. Washington, DC: American Psychological Association.

Piaget, J. (1950). The psychology of intelligence. New York: Harcourt Brace.

Robbins, J. (1995). Levels of learning in Orff SPIEL. Bulletin of the Council for Research in Music Education, 123, 47-53.

Rodwell, M.K., \& Byers, K.V. (1997). Auditing constructivist inquiry: Perspectives of two stakeholders. Qualitative Inquiry, 3(1), 116-135.

Rogoff, B. (1990). Apprenticeship in thinking. New York: Oxford University Press.

Roschelle, J. (1992). Learning by collaborating: convergent conceptual change. The Journal of the Learning Sciences, 2(3), 235-276.

Roschelle, J., \& Teasley, S.D. (1995). The construction of shared knowledge in collaborative problem solving. In C.E. O’Malley (Ed.), Computer-supported collaborative learning (pp. 69-197). Berlin: Springer-Verlag.

Rosenshine, B., Froelich, H., \& Fakhouri, I. (2002). Systematic instruction. In R. Colwell \& C. Richardson (Eds.), The new handbook of research on music teaching and learning (pp. 299-314). Oxford: Oxford University Press.

Rossman, G.B., \& Rallis, S.F. (2003). Learning in the field: An introduction to qualitative research. Thousand Oaks, CA: Sage Publications.

Roulston, K., Legette, R., DeLoach, M., Buckhalter-Pittman, C., Cory, L., \& Grenier, R. (2005). Education: Mentoring and community through research. Research Studies in Music Education, 25, 1-23.

Rousseau, C.K. (2004). Shared beliefs, conflict, and a retreat from reform: The story of a professional community of high school mathematics teachers. Teaching and Teacher Education, 20, 783-796.

St. John, P.A. (2006). Finding and making meaning: Young children as musical collaborators. Psychology of Music, 34(2), 219-237.

Scheib, J.W. (2006). Policy implications for teacher retention: Meeting the needs of the dual identities of arts educators. Arts Education Policy Review, 107(6), 5-10.

Schwandt, T. (2000). Three epistemological stances for qualitative inquiry: Interpretivism, hermeneutics, and social constructionism. In N.K. Denzin \& Y.S. Lincoln (Eds.), Handbook of qualitative research ( $2^{\text {nd }}$ ed., pp. 191-213). 
Thousand Oaks, CA: Sage.

Schwartz, D.L., \& Bransford, J.D. (1998). A time for telling. Cognition and Instruction, 16(4), 475-522.

Seidman, I. (2006). Interviewing as qualitative research. New York: Teachers College Press.

Shuler, S. C. (1995). The impact of the national standards on the preparation, in-service professional development, and assessment of music teachers. Arts Education Policy Review, 96(3), 2-14.

Sink, P. (2002). Behavioral research on direct music instruction. In R. Colwell \& C. Richardson (Eds.), The new handbook of research on music teaching and learning (pp. 315-326). Oxford: Oxford University Press.

Stanley, A.M. (2008). Rose and Giancarlo: Evidence of and for collaboration. In B. Roberts (Ed.), Sociological explorations (pp. 337-352). St. Johns, NL: The Binder's Press.

Stigler, J.W., \& Hiebert, J. (1997). Understanding and improving classroom mathematics instruction: An overview of the TIMSS video study. Phi Delta Kappan, 79(1), $14-21$.

van Boxtel, C., van der Linden, J., \& Kanselaar, G. (2000). Collaborative learning tasks and the elaboration of conceptual knowledge. Learning and Instruction (10)4, 311-330.

Vygotsky, L.S. (1978). Mind in society (M. Cole, V. John-Steiner, S.Scribner, \& E. Souberman, Eds.).Cambridge, MA: Harvard University Press.

Wenger, E. (1998). Communities of practice: Learning, meaning, and identity. Cambridge: Cambridge University Press.

Wiggins, G., \& McTighe, J. (2005). Understanding by design ( $2^{\text {nd }}$ ed.). Alexandria, VA: Association for Supervision and Curriculum Development.

Wiggins, J.H. (2000). The nature of shared musical understanding and its role in empowering independent musical thinking. Bulletin of the Council for Research in Music Education, 143, 65-90.

Yamagata-Lynch, L.C. (2001). Community of practice: What is it, and how can we use this metaphor for teacher professional development?. In Annual proceedings of selected research and development practice papers presented at the National Convention of the Association for Educational Communications and Technology. Atlanta, GA: November 8-12, 2001. 
Younker, B.A. (2003). The nature of feedback in a community of composers. In M. Hickey, (Ed.), Music composition in the schools: A new horizon for music education (pp. 233-242). Reston, VA: MENC: The National Association for Music Education.

Younker, B.A. \& Burnard, P. (2008). Investigating children's musical interactions within the activities systems of group composing and arranging: An application of Engestrom's Activity Theory. International Journal of Educational Research, 47(4), 60-74.

Younker, B.A., \& Burnard, P. (2004). The notion of collaboration in group composition: An analysis within and across young composers aged 10-12 years. Paper presented at The Eighth International Conference on Music Perception and Cognition, Evanston, IL, August.

Zeek, C., Foote, M., \& Walker, C. (2001). Teacher stories and transactional inquiry: Hearing the voices of mentor teachers. Journal of Teacher Education, 52(5), 377385. 